\title{
Hydrogen diffusion and hydride formation in grain boundary rich magnesium
}

\author{
Dissertation
}

zur Erlangung des mathematisch-naturwissenschaftlichen Doktorgrades „Doctor rerum naturalium" der Georg-August-Universität Göttingen

im Promotionsprogramm ProPhys der Georg-August University School of Science (GAUSS)

vorgelegt von

Magnus Hamm

aus Kürten

Göttingen, 15.05.2018 
Betreuungsausschuss

Prof. Dr. Astrid Pundt

Institut für Materialphysik, Georg-August-Universität Göttingen

Prof. Dr. Reiner Kirchheim

Institut für Materialphysik, Georg-August-Universität Göttingen

Mitglieder der Prüfungskommission:

Referentin: Prof. Dr. Astrid Pundt

Institut für Materialphysik, Georg-August-Universität Göttingen

Koreferent: Prof. Dr. Reiner Kirchheim

Institut für Materialphysik, Georg-August-Universität Göttingen

Weitere Mitglieder der Prüfungskommission:

Prof. Dr. Cynthia A. Volkert

Institut für Materialphysik, Georg-August-Universität Göttingen

Prof. Dr. Vasily Moshnyaga

I. Physikalisches Institut, Georg-August-Universität Göttingen

Prof. Dr. Hans-Christian Hofsäss

II. Physikalischen Institut, Georg-August-Universität Göttingen

Prof. Dr. Michael Seibt

IV. Physikalisches Institut, Georg-August-Universität Göttingen

Tag der mündlichen Prüfung: 


\section{Contents}

$\begin{array}{ll}\text { 1. Motivation } & 1\end{array}$

\begin{tabular}{lll}
\hline 2. Thermodynamics and kinetics of metal-hydrogen systems & 7
\end{tabular}

2.1. The $\mathrm{Mg}-\mathrm{H}$ system $\ldots \ldots \ldots \ldots \ldots \ldots$

2.1.1. The thermodynamics of $\mathrm{Mg}-\mathrm{H}$ bulk $\ldots \ldots \ldots \ldots$

2.1.2. The thermodynamics of $\mathrm{Mg}-\mathrm{H}$ thin films. . . . . . . . . . 11

2.1.3. The electric properties of $\mathrm{Mg}-\mathrm{H}$ (in bulk and thin films) . . . 13

2.1.4. Kinetic steps of the $\mathrm{H}$ uptake in $\mathrm{Mg} \ldots \ldots \ldots \ldots . \ldots . . \ldots 14$



2.2.1. Fick's laws of diffusion . . . . . . . . . . . . . . . . 24

2.2.2. Substitutional and interstitial diffusion . . . . . . . . . 26

$2.2 .3 . \quad$ Grain boundary diffusion . . . . . . . . . . . . . . . . . . 29

2.2.4. Diffusion in polycrystals . . . . . . . . . . . . . . 34

2.3. Kinetic models for solid state transformations . . . . . . . . . . . 39

3. Applied experimental methods and developed algorithms 45

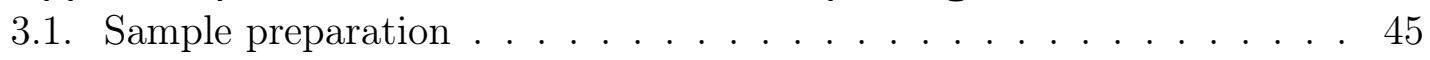

3.1.1. Argon-ion beam sputtering . . . . . . . . . . . . . . 46

3.1.2. Pulsed laser deposition . . . . . . . . . . . . . . . . . 46

3.2. Gas volumetry . . . . . . . . . . . . . . . . . . . . 49

3.2.1. Gas volumetry setup . . . . . . . . . . . . . . . . 49

3.2.2. Theoretical description of a gas volumetry measurements . . . 50

3.2.3. Developed algorithm to evaluate $D$ from gas volumetry mea-

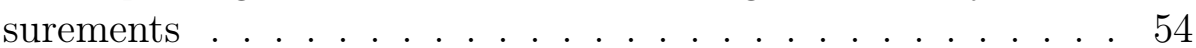

3.3. Resistance measurement . . . . . . . . . . . . . . . 56

3.3.1. Setup . . . . . . . . . . . . . . . . 57

3.3.2. Theoretical models developed and applied to evaluate resistance data . . . . . . . . . . . . . . . . . . . . 57

3.3.3. Developed evaluation algorithm for resistance data . . . . . . 61

3.4. Electron microscopy $\ldots \ldots \ldots \ldots \ldots \ldots$

3.4.1. TEM: in situ hydrogen loading . . . . . . . . . . . . 66

3.5. X-ray diffraction techniques $\ldots \ldots \ldots \ldots$. . . . . . . . 68

3.5.1. Analysis of grain structure and grain size . . . . . . . . . 69

3.5.2. XRD: in situ hydrogen loading . . . . . . . . . . . . 70 
3.6. Finite-element simulations . . . . . . . . . . . . . . . . 71

3.6.1. Simulation setup . . . . . . . . . . . . . . . 72

3.6.2. Data evaluation . . . . . . . . . . . . . . . . . 73

4. Structural characterization and individual discussion of $\mathrm{Mg}$ thin films $\begin{array}{ll}\text { upon hydrogen loading } & 77\end{array}$

4.1. Initial film morphology . . . . . . . . . . . . . . . . . . . . . . 77

4.2. Changes during hydrogen loading . . . . . . . . . . . . . . . . 82

4.3. Film morphology after hydrogen loading . . . . . . . . . . . . . . . . 88

5. Measurement results and individual discussion of diffusion coeffi$\begin{array}{ll}\text { cients of hydrogen in nanocrystalline } \mathrm{MgH}_{2} & 91\end{array}$

5.1. Evaluation of the limiting kinetic process . . . . . . . . . . . . . . 91

5.1.1. (1D) diffusion limited measurements . . . . . . . . . . . . . 93

5.1.2. Measurements with different limiting processes . . . . . . . . . . 95

5.2. Results of overall system diffusion coefficient measurements . . . . . . 98

5.3. Results of the FEM simulations . . . . . . . . . . . . . . . . . 103

6. Global discussion of the experimental results 107

6.1. Structural changes in Mg thin films by hydride formation . . . . . . . 107

6.1.1. Film structure after preparation . . . . . . . . . . . . . 107

6.1.2. Influence of hydride formation: internal stresses and their con-

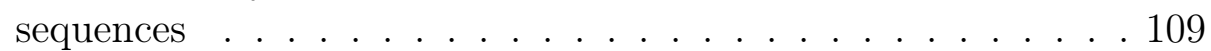

6.2. Comparison of FEM simulations with literature . . . . . . . . . . . 114

6.2.1. Comparison with theory of grain boundary diffusion . . . . . . 115

6.2.2. Comparison with literature data of $\mathrm{H}$ diffusion in the $\mathrm{Mg}-\mathrm{H}$

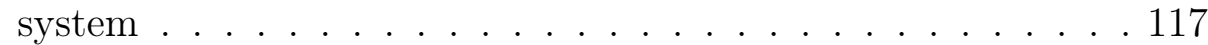

6.3. Influence of grain boundaries on $\mathrm{H}$ diffusion in $\mathrm{MgH}_{2}$. . . . . . . . . 121

6.3.1. Influence of grain size and structure . . . . . . . . . . . . . . 123

6.3.2. Influence of Fe content . . . . . . . . . . . . . . . . . . . 124

6.3.3. Influence of changes in $\mathrm{H}$ concentration in grain boundaries in

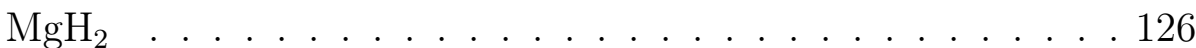

\begin{tabular}{ll}
\hline 7. Summary and outlook & 135
\end{tabular}

Appendix A. Additions to Experimental methods I

Appendix B. Additional results III

B.1. Structural characterization of $\mathrm{Mg}$ thin films upon hydrogen loading . IV

B.1.1. Film thickness changes upon hydrogen loading . . . . . . . . IV

B.1.2. XRD measurements of $\mathrm{Mg}$ thin films . . . . . . . . . . . V V

B.1.3. EELS measurements of a palladium/magnesium interlayer . . VIII

B.2. Measurement results of diffusion coefficients of hydrogen in nanocrys-

talline $\mathrm{MgH}_{2} \ldots \ldots \ldots \ldots \ldots \ldots \ldots$ IX

B.2.1. Evaluation of the limiting kinetic process . . . . . . . . . IX 
B.2.2. Results of overall system diffusion coefficient measurements . . XI

C. Bibliography

XIII

D. Danksagung XXXV 



\section{Motivation}

Energy drives our everyday life. This is true metaphorically and literally. Every transport of ourselves and of goods, brought to us, needs energy to be moved. Today at least $95 \%$ of this energy comes from non-renewable fossil fuels (e.g. gasoline, diesel, natural gas) [1, 2, 3]. Worldwide, the transportation sector consumes about $25 \%$ of the overall delivered energy [2]. For Germany the share of the transport sector is even higher at $30 \%$ [3. In addition, the energy demand of the transport sector in Germany was the only one to increase from 1990 to 2015. While the delivered energy consumed in the transport sector increased from 2379 PJ to 2619 PJ (an increase of about 10\%), the overall energy consumption in Germany decreased by $6.3 \%[3]$. Because the transport sector is so dependent on fossil fuel, the growing energy demand leads to several problems for humans and earth. Growing $\mathrm{CO}_{2}$ concentrations as a result of the combustion of fossil fuels, are a main factor of climate change, leading to e.g. rising sea levels [4, 5, 6. Furthermore, particulate matter pollution is known to cause health problems for humans and animals [7, 8, 9]. The most direct way to reduce the dependency on fossil fuels of the transport sector is to substitute internal combustion engines (ICE) with electric engines. The electric engine does not exhaust $\mathrm{CO}_{2}$ and, in addition, the overall system efficiency is much higher than for the ICE. Electric vehicles (EV) have energy efficiencies of between $50 \%$ to $70 \%$, which is two times the efficiency of ICE vehicles of about $20 \%$ to $30 \%$ [1, 10]. The large variance of the energy efficiency of EV originates from the different possibilities to provide the EV with electricity. Today, two possible solutions are economically viable. First, the engine can be powered by electricity stored in batteries. This has a high energy efficiency of about $70 \%$ [10]. Second, the electricity can be produced on-board by a fuel cell (FC) from hydrogen (or natural or biogas). The energy efficiency of this setup is somewhat lower (about $50 \%$ to $60 \%$ ) [1, 10]. However, it has two advantages. First, recharging hydrogen for the vehicle is done in minutes, while the recharging of the batteries takes at least $20 \mathrm{~min}$ to $30 \mathrm{~min}$ [11. Second, the energy density of an FC system is higher than that of today's batteries, leading to higher gravimetric storage density of $\mathrm{FC}$ systems ${ }^{1}$ [1, 10]. Hence, a fuel cell electric vehicle (FCEV) does not have to increase the weight of its powertrain as much as a battery electric vehicle (BEV) does to increase its rang $\mathrm{2}^{2}$ FCEV are therefore especially interesting for long range applications,

\footnotetext{
${ }^{1}$ Both, batteries and FC systems have lower gravimetric storage densities than ICE vehicles.

${ }^{2}$ While BEV and FCEV are often discussed as two separate possibilities, the technology can be combined. Offer et al. showed that this may be the most economically efficient solution [12].
} 


\section{Motivation}

e.g. trucks or trains.

Two main factors restrict the application of FCEV today. First, it is difficult to refill the vehicle with hydrogen because of the scarcity of fuel stations. However, this is a more political/economical problem than a scientific one. The second factor is connected to the first. The storage of hydrogen needs additional research. This includes the on-board storage, as well as the storage at fuel stations and for delivery trucks. Today, hydrogen is either stored under high pressures (either at $20 \mathrm{MPa}$ or at $80 \mathrm{MPa}$ ) or liquefied [10, 13, 14, 15, 16]. A big drawback of both methods is that both require additional energy to reach the storage state. High pressure hydrogen storage needs about $15.5 \%$ of its lower heating value (LHV) to reach $80 \mathrm{MPa}$ [17]. To liquefy hydrogen one needs even more energy, today about $30 \%$ of the LHV [17, 10]. New hydrogen storage materials have been researched intensively. The most promising materials can be divided into rechargeable (organic) hydrides, surface adsorbtion and (metal) hydrides [13, 14, 15, 16, 10]. These methods not only have to be competitive with high pressure and liquid storage, but with real life economical requirements. Guidelines for these are given by the U.S. Department of Energy (DOE) [18]. The DOE gives the following goals for on-board hydrogen storage for 2020: A gravimetric density of $5.5 \mathrm{wt} \% \mathrm{H}_{2}$ and a cost of $\$ 333 / \mathrm{kgH}_{2}{ }^{3}$. Several materials exist which can theoretically reach these goals, one of the most promising being magnesium.

Magnesium has a theoretical storage density of $7.6 \mathrm{wt} \% \mathrm{H}_{2}$ [19, 20, 21]. Further, it is cheap ${ }^{4}$, widely available and non toxic. Because of this, magnesium is regarded as a promising hydrogen storage material and has been researched as such for decades [23, 24, 25]. However, some properties still prevent real world application. One is the high temperature of about $300{ }^{\circ} \mathrm{C}$ needed to release the hydrogen from the magnesiumdihydride [17, 25, 26]. While this stability makes the hydride a very safe storage material, it increases the energy demands of the overall system. Additional energy of $30.8 \%$ of the LHV is needed to release the hydrogen [17]. The second major challenge lies in the kinetics of the formation (and decomposition) of the magnesiumdihydride from magnesium and hydrogen. The formation (as well as the decomposition) can be divided into three steps [27, 28]):

- The surface (adsorption and) dissociation of hydrogen

- The hydrogen absorption into the magnesium

- the nucleation and growth of the hydride phase

Each step contains challenges, which need to be overcome to improve the overall

\footnotetext{
${ }^{3}$ The long-term goals are $7.5 \mathrm{wt} \% \mathrm{H}_{2}$ and $\$ 216 / \mathrm{kgH}_{2}$.

${ }^{4}$ The price has been between $\$ 2 \mathrm{~kg}^{-1}$ to $\$ 3 \mathrm{~kg}^{-1}$ for the last years [22].
} 
kinetics of the system [23, 25, 20, 29]. First, hydrogen needs to adsorb to the surface and dissociate from molecular hydrogen to atomic hydrogen. For magnesium this is hindered by the low dissociation of hydrogen [30, 31]. Furthermore, magnesium forms a magnesiumoxide layer on top [32, 33. The oxide prevents the absorption of the atomic hydrogen into the material. Both steps, dissociation and absorption, can be improved by the addition of hydrogen catalysts on the surface [31, 34, 35]. Hydrogen catalysts improve the dissociation of atomic hydrogen. At the same time the catalyst can prevent the oxidation of the underlying magnesium, which allows hydrogen to absorb more easily. An often used example of a catalyst is palladium [36, 37, 38]. Furthermore, the absorption of hydrogen into the magnesium can be improved by high temperatures [39, 40]. The increased temperatures lead to cracks in thick oxide layers as the magnesium expands and the decomposition of the oxide in vacuum for temperatures above $623 \mathrm{~K}$.

While catalysts and high temperatures offer ways to overcome issues during the first two kinetic steps, the case is more complex for the third step. It is this step that generally limits the overall kinetics [41, 42]. The reason for this is the so called "blocking layer" [24, 43]. The blocking layer is a closed layer of magnesiumhydride which forms on the surface of magnesium. Once it is formed, the further hydride formation is limited by the diffusion of hydrogen through the magnesiumdihydride. This process is known to be very slow with literature values of the diffusion coefficient of hydrogen in magnesiumdihydride ranging from $10^{-16} \mathrm{~m}^{2} \mathrm{~s}^{-1}$ to $10^{-29} \mathrm{~m}^{2} \mathrm{~s}^{-1} 44$, 45]. Uchida et al. described the formation of the blocking layer in more detail [44]. He found that the formation of the hydride occurs at the surface of the magnesium, opposite to an earlier model by Vigeholm et al. [46]. The nuclei density depends on the driving force. A higher driving force (e.g. a higher overpressure over the formation pressure) leads to a higher nuclei density and a closed blocking layer is formed earlier. By lowering the hydrogen pressure, Uchida et al. were able to hydride thin films of a thickness up to $2800 \mathrm{~nm}$ [44]. However, bulk magnesium for storage applications will most likely still form a closed blocking layer even at lower overpressures. The percentage of material hydrided at this point can be improved by reducing particle and grain sizes by different techniques like ball milling [19, 20, 47, 48, 49, 50]. This decreases the diffusion length the hydrogen needs to travel through the blocking layer, leading to shorter times to reach the maximum hydride volume.

In the end, it is still this diffusion through the hydride blocking layer that limits the overall kinetics of the system and thereby the application of magnesium as a storage material. A possible way to improve this process lies in grain boundaries. Grain boundaries are known to offer fast diffusion paths in many materials [51. Yet, for hydrogen the opposite effect is also known (so called "trapping" of hydrogen in defects) [52, 53, 54, 55, 56, 57]. However, trapping has mostly been found for low hydrogen concentrations in a material. Because of this and because of the low diffusion found for hydrogen in magnesiumdihydride it seems reasonable that 


\section{Motivation}

grain boundaries may improve the overall diffusion. The influence of a single grain boundary on the overall diffusion was described by Fisher [58]. His work was the basis of much work after him [59, 60, 61, 62, 63. Later, Harrison discussed the influence of grain boundaries in polycrystals, leading to the division into the so called Harrison regimes [64]. All of these models describe grain boundaries as fast diffusion paths in the surrounding volume and lay the theoretical foundation to describe their influence on diffusion processes. The diffusion in grain boundaries themselves is still the subject of a lot of research [65, 66, 67, 68]. It was found that factors like the diffusion direction in the grain boundary and the internal structure of the grain boundary influence the diffusion coefficient.

The aim of this thesis is to unravel the role of grain boundaries on the hydride formation in $\mathrm{Mg}-\mathrm{H}$. This includes deducing the contribution of grain boundaries on the overall diffusion kinetics in the dihydride phase, which is limited by the blocking layer. It is accompanied by the study of microstructural changes, necessary for the description by Harrison regimes. Further, the impact of a ternary compound (Fe) on the overall diffusion kinetics is investigated. Iron is chosen because it does not solve in magnesium (see reference [69]) and may change the grain boundary structure and diffusion.

This thesis is divided into seven chapters. In the following chapter 2 the theoretical background for this work is presented. Chapter 2.1 describes the magnesiumhydrogen system, including the thermodynamic, electrical and kinetic properties for bulk system and thin films. Thereafter, the basics of diffusion and, especially, grain boundary diffusion are explained in chapter 2.2. It describes in more detail the models of Fisher and Harrison. The last section, 2.3, of chapter 2 describes kinetic models for the solid state transformation. The experimental methods applied and algorithms developed for this work are discussed in chapter 3 . First, the sample preparation is described in chapter 3.1. Afterwards, two sections describe the gas volumetry (chapter 3.2) and the resistance measurement (chapter 3.3). Chapter 3 continues with the description of the electron microscopy and X-ray diffraction methods utilized (see chapter 3.4 and chapter 3.5). Both include in situ studies of the hydride formation. The final section of chapter 3 describes finite element (FEM) simulations performed for this work. These allow to study the influence of the grain boundary diffusion and the volume diffusion on the overall diffusion of the system. Chapter 4 describes the grain morphology of the magnesium thin films studied in this work. It includes the initial morphology, as well as changes during the hydride formation. Chapter 5 presents the results of the diffusion measurements. First, the limiting kinetic process is evaluated to confirm that the system kinetics are limited by the diffusion of hydrogen (see chapter 5.1). Afterwards, in chapter 5.2 the measured diffusion coefficients are collected and compared with different experimental parameters. The goal is to find possible influences on the overall diffusion of hydrogen. The final section 5.3 shows the results of the finite element simulations. Chapter 6 discusses the results. The first part in chapter 6.1 examines the changes in 
grain morphology as presented in chapter 4. Afterwards, the results of the diffusion coefficient studies are evaluated (see chapter 6.2 and chapter 6.3). The influence of the different parameters can be explained by taking the FEM results into account. The final chapter 7 is a summary of the work and of the results found. 



\section{Thermodynamics and kinetics of metal-hydrogen systems}

This chapter will lay the basic theoretical background. At first, the magnesiumhydrogen system will be introduced (see section 2.1). The first part introduces the thermodynamics to transform magnesium $(\mathrm{Mg})$ to magnesiumdihydride $\left(\mathrm{MgH}_{2}\right)$ and discusses the two phases in bulk systems and in thin films. Furthermore the electrical properties of the two phases will be given. Following that, the kinetic process will be discussed, introducing the different kinetic steps during the transformation. This section includes also a collection of diffusion coefficients as published over the years in the literature. The following section 2.2 introduces the diffusion in solids, giving further insight into the different kinds of diffusion coefficients. This starts by introducing Fick's first and second law and the difference of interstitial and substitutional diffusion. Thereafter, theories about grain boundary diffusion and diffusion in polycrystals are discussed. These theories are necessary to separate the influence of grain boundaries and grains on the overall diffusion and therefore on the diffusion coefficients measured in this work. The final section of this chapter discusses kinetic models for phase transformation in the solid state (section 2.3). These models allow to determine the limiting kinetic process of a transformation. Hence, they reveal if a measurement is limited by kinetic processes other than the hydrogen diffusion. If this is the case an evaluation of the diffusion coefficient is futile.

\subsection{The $\mathrm{Mg}-\mathrm{H}$ system}

In the following the thermodynamic and electrical properties of the $\mathrm{Mg}-\mathrm{H}$ system will be introduced. At first the thermodynamic properties of the bulk system will be presented. Thereafter, a chapter presents the changes found in thin $\mathrm{Mg}-\mathrm{H}$ films. The electric properties of the bulk system and thin films are then presented together in one chapter. Afterwards, the focus will be on the kinetic steps of the phase transformations in the system. This is an important research focus, because it is the kinetic behavior that limits the practical application. In particular the so called "blocking layer" prohibits practical applications. The blocking layer is a $\mathrm{MgH}_{2}$ layer that prevents further dihydride formation [46, 44]. This is due to the 
low diffusivity of $\mathrm{H}$ through the $\mathrm{MgH}_{2}$.

\subsubsection{The thermodynamics of $\mathrm{Mg}-\mathrm{H}$ bulk}

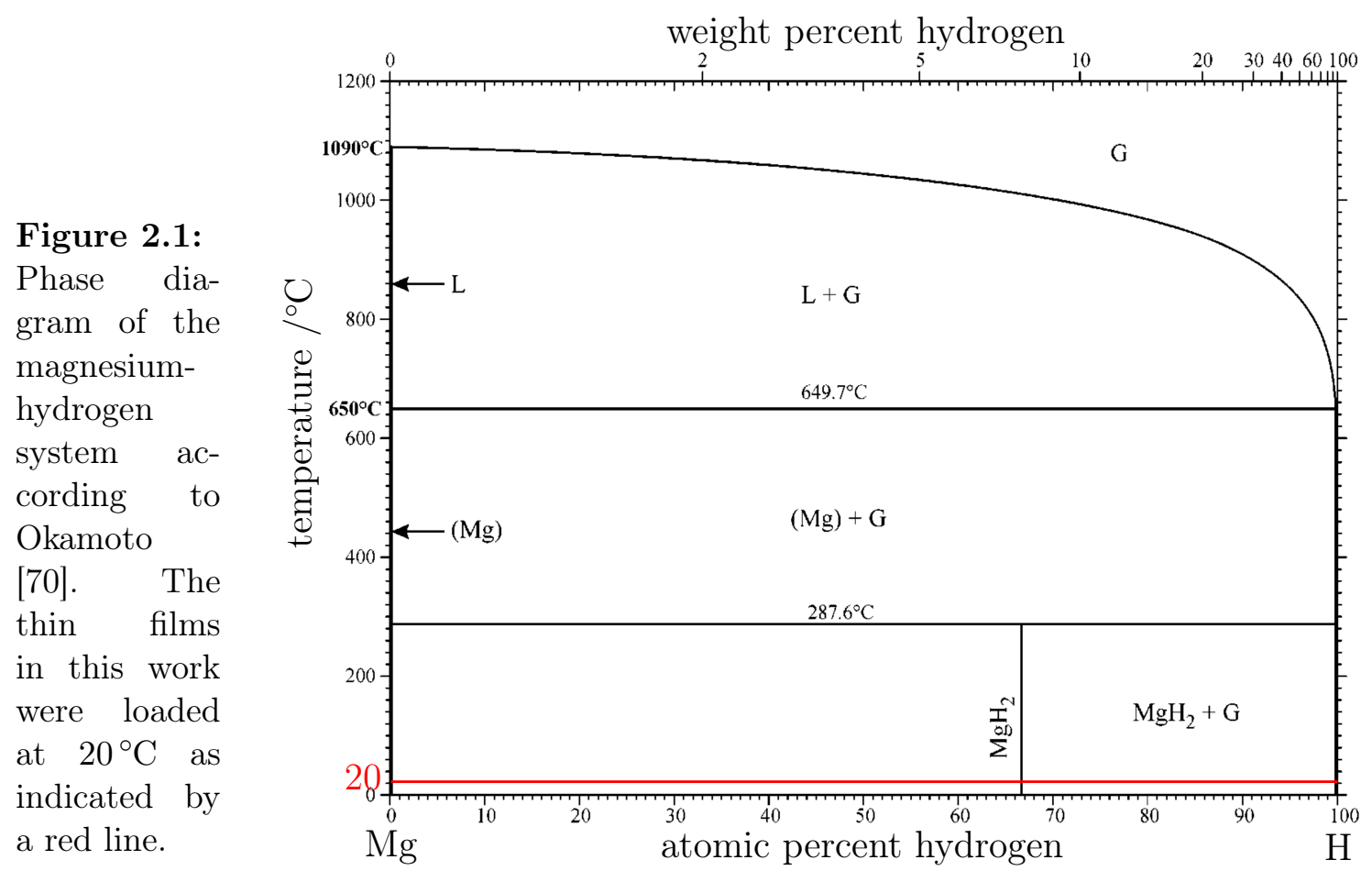

Figure 2.1 shows the bulk phase diagram of the $\mathrm{Mg}-\mathrm{H}$ system at $25 \mathrm{MPa}$ according to Okamoto [70]. The two most important phases for this work are the $\alpha$-phase, in which a small amount of hydrogen is stored in solid solution before the $\beta$-phase is formed. In figure 2.1 the $\alpha$-phase is labeled as $\mathrm{Mg}$ and the $\beta$-phase is labeled as $\mathrm{MgH}_{2}$. While the $\alpha$ - and $\beta$-phase are thermodynamically stable phases, additional phases have been discussed in the literature [24, 21, 171, 72]. A metastable $\gamma$-phase is known to form at high pressure conditions. It will be discussed shortly in regard to high stresses that are known to arise in thin films during hydrogen loading 73 . (compare section 2.1.2). In addition the literature discusses a $\delta$-phase with a distorted $\mathrm{CaF}_{2}$ structure [74] and a $\varepsilon$-phase with an $\mathrm{AlAu}_{2}$ structure [75]. Both phases are not expected to form during the moderate conditions used in this work and are excluded from the discussion. All in all, the (di-)hydride phase in this work is synonymous with the $\beta$-phase, except where it is specified otherwise. A very informative collection of the different hyride phases and their thermodynamic parameters are given in the thesis of $\mathrm{H}$. Uchida [76]. As the focus of this work is more on the kinetics of the $\mathrm{Mg}-\mathrm{H}$ system the thermodynamic behavior is discussed in a shorter form.

Metallic magnesium has a hexagonal crystal structure. The lattice parameters are 


\begin{tabular}{lr||ccc|c}
\hline Phase & Structure & $\mathrm{a} / \AA$ & $\mathrm{b} / \AA$ & $\mathrm{c} / \AA$ & Ref. \\
\hline \hline$\alpha-\mathrm{Mg}$ & hexagonal $\left(P 6_{3} / m n m\right)$ & 3.21 & $=\mathrm{a}$ & 5.21 & {$[24]$} \\
\hline$\beta-\mathrm{MgH}_{2}$ & tetragonal $\left(P 4_{2} m n m\right)$ & 4.50 to 4.52 & 3.01 to 3.02 & $=\mathrm{a}$ & {$[24]$} \\
& $\left(\mathrm{TiO}_{2}\right.$ structure $)$ & 4.51 to 4.52 & 3.01 to 3.02 & $=\mathrm{a}$ & {$[72]$} \\
\hline$\gamma-\mathrm{MgH}_{2}$ & orthorhombic $($ Pbcn $)$ & 4.53 & 5.44 to 5.45 & 4.93 to 4.94 & {$[24]$} \\
& $\left(\alpha-\mathrm{PbO}_{2}\right.$ structure $)$ & 4.51 to 4.52 & 5.43 to 5.44 & 4.92 to 4.94 & {$[72]$} \\
\hline
\end{tabular}

Table 2.1.: Structural information of the $\alpha-\beta$ - and $\gamma$-phase of the magnesium-hydrogen system. Only the $\alpha$ - and $\beta$-phase are thermodynamically stable. The lattice parameters of all phases were collected by San-Martin and Manchester in 1987 [24] and by Moser et al. in 2011 [72].

given in table 2.1. The $\alpha$-phase stores some hydrogen as a solid solution. For ambient conditions the hydrogen forms clusters in hydrogen-vacancy complexes [24]. At low temperatures $(T<110 \mathrm{~K})$ hydrogen is solved in the tetrahedral lattice sites and not yet trapped by vacancies. Overall, the solubility of hydrogen is very low for the pure $\mathrm{Mg}$ phase. Stampfer et al. gave the relations between the maximum solubility $c_{H}^{\alpha \rightarrow \beta}$ of the $\alpha$-phase for a given temperature $T$ [77]:

$$
c_{H}^{\alpha \rightarrow \beta}=107 \cdot \exp \left(-\frac{6225}{T}\right) .
$$

This gives a maximal solution of $c_{H}^{\alpha \rightarrow \beta}=8.4 \cdot 10^{-8} \mathrm{H} / \mathrm{Mg}$ at $T=297 \mathrm{~K}$. When more hydrogen is added, the $\mathrm{Mg}-\mathrm{H}$ system transforms into one of the hydride phases. At ambient conditions, the transformation will be into the tetragonal $\beta$-phase. The crystal structure of the $\beta$-phase is also given in table 2.1. The $\beta$-phase is a stoichiometric phase, meaning that the concentration of hydrogen is fixed to $c_{H}^{\beta}=2 \mathrm{H} / \mathrm{Mg}$ and no additional hydrogen can be solved in the bulk [77]. For real systems this may not be completely true; for example grain boundaries may take up different amounts of hydrogen. Borgschulte et al. also showed that oxides can destabilize $\mathrm{MgH}_{2}$ to an under-stoichiometric $\mathrm{MgH}_{2-\delta}$-phase [78]. The under-stoichiometric phase was documented before by Schimmel et al. [79].

The hydride formation from a hydrogen gas atmosphere can be plotted by a pressurecomposite isotherm (called p-c-T diagram, see figure 2.2] [27]. For a fixed temperature $T$ the hydrogen pressure $p_{H}$ and the hydrogen concentration $c_{H}$ taken up by the metal can be measured (for example by the calculations in chapter 3.2.2). The resulting diagram shows a pressure plateau in the two-phase region of the $\alpha$ - and $\beta$-phase. By repeating the measurement for different temperatures, a Van't Hoff plot is created. It shows the logarithm of the hydrogen pressure plotted as function of the reciprocal temperature. The principle is sketched in figure 2.2. The Van't Hoff plot allows the evaluation of the enthalpy change $\Delta H$ and entropy change $\Delta S$ 


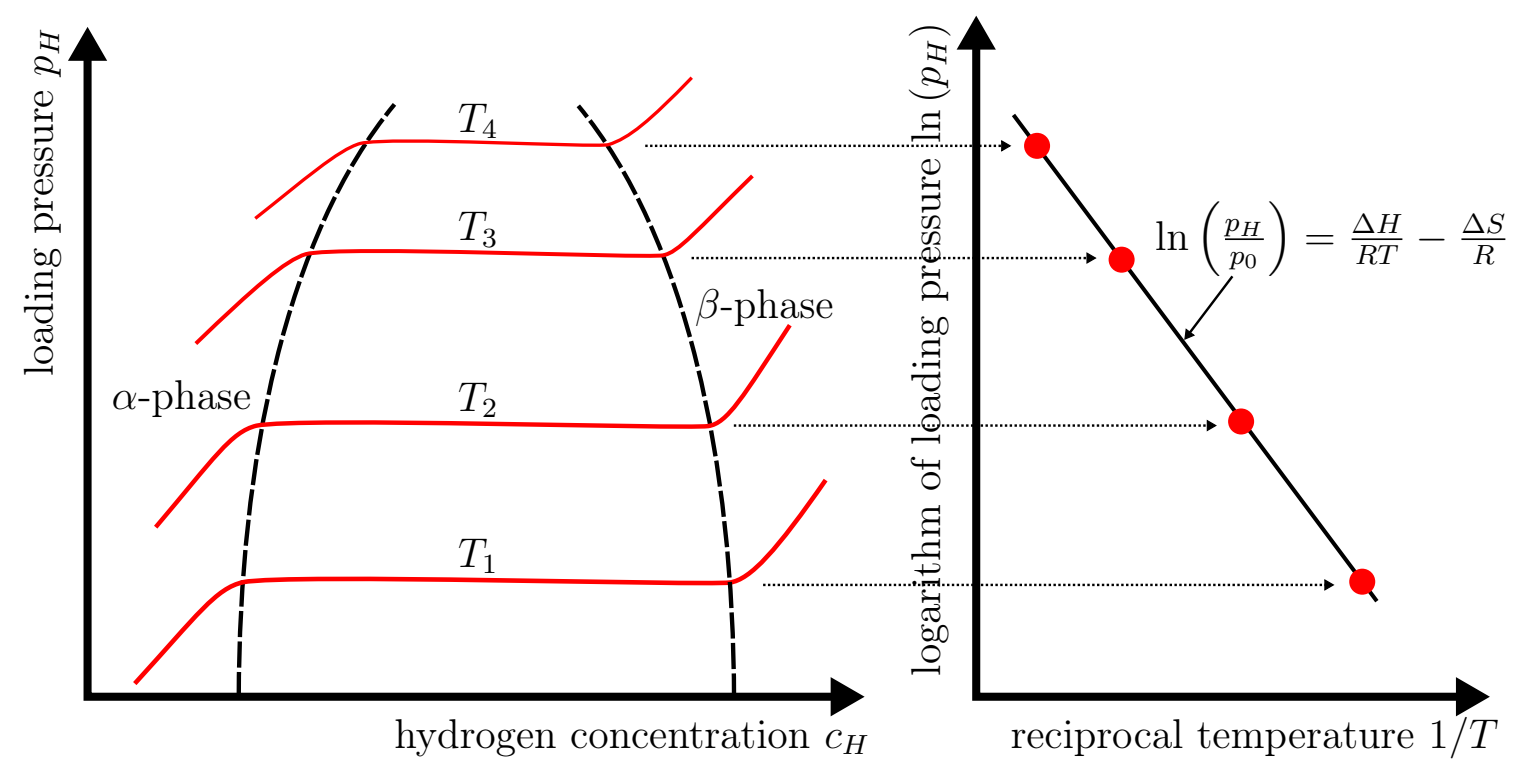

Figure 2.2.: Example of a p-c-T diagram and the resulting Van't Hoff plot. The p-c$\mathrm{T}$ diagram plots the hydrogen concentration $c_{H}$ in the metal as function of the loading hydrogen pressure $p_{H}$. The plateau of a single isotherm gives the two-phase region width at a given temperature $T$. Measuring isotherms at different temperatures $T_{1}<T_{2}<$ $T_{3}<T_{4}<\ldots$ allows creating a Van't Hoff plot of the logarithm of the loading pressure as function of the reciprocal temperature. From this one can evaluate the enthalpy - and entropy change of the phase transformation from $\alpha$ - to $\beta$-phase.

during the phase transformation [80, 13]:

$$
\ln \left(\frac{p_{H}}{p_{0}}\right)=\frac{\Delta H}{R} \frac{1}{T}-\frac{\Delta S}{R},
$$

where $p_{0}$ is the standard pressure and $R$ the gas constant. These entropy and enthalpy changes may be different for the absorption and desorption of hydrogen, resulting in different plateau pressures. The entropy change $\Delta S$ comes mostly from the dissociation of hydrogen from the $\mathrm{H}_{2}$ molecule in the gas atmosphere to the atomically dissolved hydrogen atom in the metal hydride. After Fukai, the standard entropy of hydrogen is approximately $S_{0}=130 \mathrm{JK}^{-1} \mathrm{~mol}^{-1}$ [27]. Therefore, the entropy change can be estimated as $\Delta S \approx-130 \mathrm{JK}^{-1}\left(\mathrm{molH}_{2}\right)^{-1}$ for all metal-hydrogen systems. For the magnesium-hydrogen system this fits well to the measured changes in entropy. Vigeholm et al. measured an entropy change of $\Delta S=-126 \mathrm{JK}^{-1}\left(\mathrm{molH}_{2}\right)^{-1}$ [81], while Klose and Stuke measured $\Delta S=-146.1 \mathrm{JK}^{-1}\left(\mathrm{molH}_{2}\right)^{-1}$ [82. Other groups measured values between these two, around $\Delta S=-130 \mathrm{JK}^{-1}\left(\mathrm{molH}_{2}\right)^{-1}$ (e.g. references [24, 77, 30, 83]). The enthalpy change for the absorption was found to be about $\Delta H^{a b s} \approx-70 \mathrm{~kJ}\left(\mathrm{molH}_{2}\right)^{-1}$ in the bulk system [78, 81. For the desorption enthalpy change most groups measured values of approximately $\Delta H^{\text {des }}=$ $(-75 \pm 5) \mathrm{kJ}\left(\mathrm{molH}_{2}\right)^{-1}$ in bulk systems (see references [24, 77, 78, 30, 83]).

With the literature data and equation 2.1 the absorption pressure expected in bulk 
systems can be calculated for a temperature of $T=297 \mathrm{~K}$ :

$$
p_{H}=101325 \cdot \exp \left(\frac{-70000}{8.31446 \cdot 297}-\frac{-130}{8.31446}\right) \mathrm{Pa} \frac{\mathrm{J} \mathrm{K} \mathrm{mol}}{\mathrm{J} \mathrm{K} \mathrm{mol}} \approx 0.3 \mathrm{~Pa}
$$

Furthermore, equation 2.1 allows estimating the necessary temperature to release hydrogen from the hydride at ambient pressure using the values for $\Delta H^{\text {des }}$ and $\Delta S$ given above $\left(\Rightarrow p_{H} / p_{0} \approx 1\right)$ :

$$
\begin{aligned}
\ln (1) & =\frac{-75000}{8.31446 \cdot T^{d e s}} \frac{\mathrm{J} \mathrm{K} \mathrm{mol}}{\mathrm{J} \mathrm{K} \mathrm{mol}}-\frac{-130}{8.31446} \frac{\mathrm{J} \mathrm{K} \mathrm{mol}}{\mathrm{J} \mathrm{K} \mathrm{mol}}, \\
\Leftrightarrow T^{\text {des }} & =\frac{75000}{130} \frac{\mathrm{J} \mathrm{K} \mathrm{mol}}{\mathrm{J} \mathrm{mol}} \approx 577 \mathrm{~K} .
\end{aligned}
$$

This shows the thermodynamic stability of the $\beta$-phase, as it needs low pressures to form at $T=20^{\circ} \mathrm{C}$ or high temperatures of about $300^{\circ} \mathrm{C}$ to dehydride at 1 bar hydrogen pressure.

\subsubsection{The thermodynamics of $\mathrm{Mg}-\mathrm{H}$ thin films}

In thin films hydrogen loading leads to strains and stresses, which change the thermodynamics of the $\mathrm{Mg}-\mathrm{H}$ system. In the last few years studies on palladiumand niobium thin films showed drastic influences of the stress state on the thermodynamic equilibrium [84, 85, 86]. It was shown that stresses decrease the critical temperature of the two-phase region, leading to a smaller two phase region at a fixed temperature T. Furthermore, stresses lead to a higher plateau pressure for hydride formation in thin films compared with bulk systems at the same temperature.

Thin films are adhered to their substrate, which prevents the expansion in the inplane direction. This leads to in-plane strains and stresses [73, 87, 88]. Additionally, the expansion in the out-of-plane direction is increased. Both effects can be calculated using linear elastic theory (see e.g. [89]) and the volume expansion of the unit cell during the hydride formation. The volume expands from the hexagonal unit cell of the magnesium $\left(V_{M g}^{\text {unit }}=3.21^{2} \cdot \sin \left(60^{\circ}\right) \cdot 5.21 \AA^{3} \approx 46.49 \AA^{3}\right)$ to the larger tetragonal unit cell of the $\mathrm{MgH}_{2} \beta$-phase $\left(V_{M g H 2}^{\text {unit }}=4.51^{2} \cdot 3.01 \AA^{3} \approx 61.22 \AA^{3}\right)$ (see table 2.1). Because of the low solubility in the $\alpha$-phase any additional expansion of its unit cell because of hydrogen uptake can be ignored. The relative expansion during the phase transformation is given by:

$$
\frac{\Delta V^{\text {unit }}}{V_{M g}^{\text {unit }}}=\frac{V_{M g H 2}^{\text {unit }}}{V_{M g}^{\text {unit }}}-1=\frac{61.22}{46.49}-1 \approx 0.32 .
$$

The increased overall out-of-plane expansion $\varepsilon_{t o t}$ for a thin film can be calculated. For the thin films studied in this work ( $\mathrm{Mg}$ thin films with (0001) out-of-plane 
direction and $\mathrm{MgH}_{2}$ thin films with an (110) out-of-plane direction) the necessary equations were given by $\mathrm{H}$. Uchida [76, 90]:

$$
\begin{aligned}
\varepsilon_{t o t}^{M g} & =\left(1+2 \frac{C_{13}}{C_{33}}\right) \cdot \varepsilon_{0}, \\
\varepsilon_{t o t}^{M g H 2} & =\left(1+\frac{2 C_{11}+C_{66}+3 C_{13}}{2 C_{11}+C_{66}+C_{13}}\right) \cdot \varepsilon_{0} .
\end{aligned}
$$

$\varepsilon_{0}$ can be calculated as one third of the relative volume expansion $(\Delta v / v) / 3 \approx$ 0.11. $C_{x x}$ are the elastic constants of magnesium and magnesiumdihydride (see e.g. references [91] and [92]). The total out-of-plane expansion for the $\mathrm{MgH}_{2}$ film system is calculated to be: $\varepsilon_{\text {tot }}^{M g} \approx 0.25$. This is the expansion that a $\mathrm{Mg}$ thin film should show after it is fully hydrided. However, up until now no plasticity was taken into account. In a real system plasticity limits the linear elastic maximum value, because the formation of dislocations leads to lower out-of-plane expansions as shown for thin niobium films [73, 87, 88].

The influence of the stress on the thermodynamics can be shown by comparing the literature data of $\Delta H^{a b s}$ and $\Delta H^{\text {des }}$ with the bulk values. While thin films were not studied as extensively as bulk systems in the past, Krozer and Kasemo and Pivak et al. measured values for both [26, 30]. The desorption enthalpy change seems to be similar to the bulk values $\left(\Delta H^{\text {des }}=-71 \mathrm{~kJ}\left(\mathrm{molH}_{2}\right)^{-1}\right.$ and $\Delta H^{\text {des }}=$ $-78.3 \mathrm{~kJ}\left(\mathrm{molH}_{2}\right)^{-1}$ respectively $)$. By contrast, the absorption enthalpy change was found to be lower in both studies. Kozer and Kasemo measured a value of $\Delta H^{\text {des }}=$ $-60.7 \mathrm{~kJ}\left(\mathrm{molH}_{2}\right)^{-1}$, while Pivak measured $\Delta H^{\text {des }}=-61.6 \mathrm{~kJ}\left(\mathrm{molH}_{2}\right)^{-1}$. Pivak et al. argued that the increased hysteresis is a result of the adhesion of the thin film to the substrate, leading to additional strains and stresses and resulting in additional mechanical work. With the literature data and equation 2.1 the absorption pressure expected in thin films can be calculated for a temperature of $T=297 \mathrm{~K}$ :

$$
p_{H}=101325 \cdot \exp \left(\frac{-61000}{8.31446 \cdot 297}-\frac{-130}{8.31446}\right) \mathrm{Pa} \frac{\mathrm{J} \mathrm{K} \mathrm{mol}}{\mathrm{J} \mathrm{K} \mathrm{mol}} \approx 12 \mathrm{~Pa} .
$$

This is about 40 times the value calculated for the bulk system in equation 2.2. Krozer and Kasemo and Pivak et al. found the same $\Delta H^{\text {des }}$ as in the bulk system, leading to the same desorption temperature $T^{\text {des }}$ calculated in 2.3 . However, a reduction of the critical temperature and therefore $T^{\text {des }}$ has been found in niobium thin film by Burlaka et al. [86].

Uchida measured the in-plane stresses in thin Mg films during hydrogen loading [76]. He obtained values of up to several hundred MPa. For thinner niobium films, stresses of ten GPa have been measured [88]. These high stresses can theoretically lead to the formation of the $\mathrm{MgH}_{2} \gamma$-phase. The $\gamma$-phase was first found experimentally during high pressure synthesis of magnesiumhydride [93]. This includes high pressure deformation of pre-formed magnesiumhydrides [94, 95, 96] and hydriding magnesium 
at high gas pressure [97. Its structure is given in table 2.1 and was confirmed by Bortz et al. [94]. The transition pressure from the $\beta$ - to the $\gamma$-phase has been studied by Cui et al. and Vajestoon et al. [98, 99]. A full pressure-temperature phase diagram was calculated by Moser et al, giving a transition pressure of about $(2 \pm 1) \mathrm{GPa}$ at $300 \mathrm{~K}[72$. Moser et al. also explain why experimentally a wide difference in formation pressures (and temperatures) is found, ranging from about $2 \mathrm{GPa}$ to $8 \mathrm{GPa}$. They calculated a minimal difference in the Gibb's energy of the two hydride phases explaining the experimentally confirmed coexistence of the two phases. In accordance with formation of the $\gamma$-phase at high pressures, and the discussed high pressures during hydrogenation of thin films, Ham et al. found the formation of the $\gamma$-phase in thin $\mathrm{Mg}$ films [71. They deposited $1.6 \mu \mathrm{m} \mathrm{Mg}$ thin films and $\mathrm{Mg}-\mathrm{Nb}$ multilayers with single-layer thicknesses of $100 \mathrm{~nm}$ and $10 \mathrm{~nm}$. The films were loaded at $0.25 \mathrm{MPa}$ and $373 \mathrm{~K}$. While the film thickness is therefore similar to the thicknesses studied in this work, the hydrogen loading was done at higher temperatures and much higher pressures.

\subsubsection{The electric properties of $\mathrm{Mg}-\mathrm{H}$ (in bulk and thin films)}

As magnesium is a metal it has a low specific resistance of $\rho_{M g}^{c}=37.8 \Omega \mathrm{nm}$ in the direction of the c-axis and $\rho_{M g}^{a}=45.3 \Omega \mathrm{nm}$ in direction of the a-axis [100]. The $\beta$ phase is insulating $(\rho>>M \Omega$ [101] $)$ with measured band gap energies of $4.3 \mathrm{eV}$ to $5.8 \mathrm{eV}$ for bulk samples [102, 103]. Giebels et al. and Westerwaal et al. studied thin films of $\mathrm{MgH}_{2}$ and $\mathrm{Mg}$ by gas-phase hydrogenography and modeled their results with the effective medium theory [101, 104]. Giebels et al. found a specific resistance of the $\mathrm{Mg}$ thin films of $\rho_{M g}=65 \Omega \mathrm{nm}$, slightly higher than the bulk values [104]. This is to be expected, as thin films have a higher surface-to-volume ratio and smaller grains, both adding to the resistance [105]. Interestingly, the specific resistance for hydrided thin Mg films was found by Giebels et al. to be relatively low, with values of $\rho_{\mathrm{MgH} 2}=10^{5} \Omega \mathrm{nm}$ [104]. In opposition to this, Westerwaal et al. found the specific resistance of directly deposited $\mathrm{MgH}_{2}$ too high to be measurable, confirming the insulating properties [101]. Hence, while deposited $\mathrm{MgH}_{2}$ films were found to be insulating, $\mathrm{MgH}_{2}$ thin films that were formed by hydriding deposited $\mathrm{Mg}$ films had much lower specific resistances. Westerwaal et al. explained this by an influence of grain boundaries as conducting paths in the case of the hydrided films. This explanation can be supported by work done on $\mathrm{TiO}_{2}$, another good insulator with the same rutile grain structure as the $\mathrm{MgH}_{2} \beta$-phase. Demetry et al. showed that smaller grain sizes (三 higher grain boundary density) leads to an increase in conductivity [106. This effect was found to not directly scale with the grain boundary density, meaning that an additional effect seems to contribute. As a possible additional influence Demetry et al. suggest a change in grain boundary segregation depending 
on the grain size (for more information about segregation see section 2.2.3). This seems to confirm the general explanation given by Westerwaal et al. for $\mathrm{MgH}_{2}$ thin films. The origin of the increased conductivity of the grain boundaries is unclear, but differences in segregation and structure of the grain boundaries may explain their improved conductivity compared to the insulating bulk $\mathrm{MgH}_{2}$.

\subsubsection{Kinetic steps of the $\mathrm{H}$ uptake in $\mathrm{Mg}$}

Magnesium offers good properties for mobile hydrogen storage, mainly a high gravimetric capacity. While the thermodynamic stability of the hydride phase is a disadvantage (especially the high desorption temperature), the main challenge for a practical application originate from the kinetics of the hydride formation [23, 25, 20, 29]. Experiments often find that much higher pressures or temperatures, than thermodynamically predicted, are necessary to hydride or dehydride magnesium samples. This indicates additional kinetic barriers. The hydride formation can generally be divided into three kinetic steps, which are schematically shown in figure 2.3 (for additional information see reference [27, 28]):

1) The surface (adsorption and) dissociation of hydrogen

2) The hydrogen absorption into the magnesium

3) The nucleation and growth of the hydride phase

Figure 2.3:

Schematic representation of the kinetic steps necessary to form a hydride from hydrogen molecules and a metal. The hydrogen needs to dissociate to atomic hydrogen, which afterwards absorbs into the metal where it nucleates to a hydride and grows.

1) hydrogen dissociation

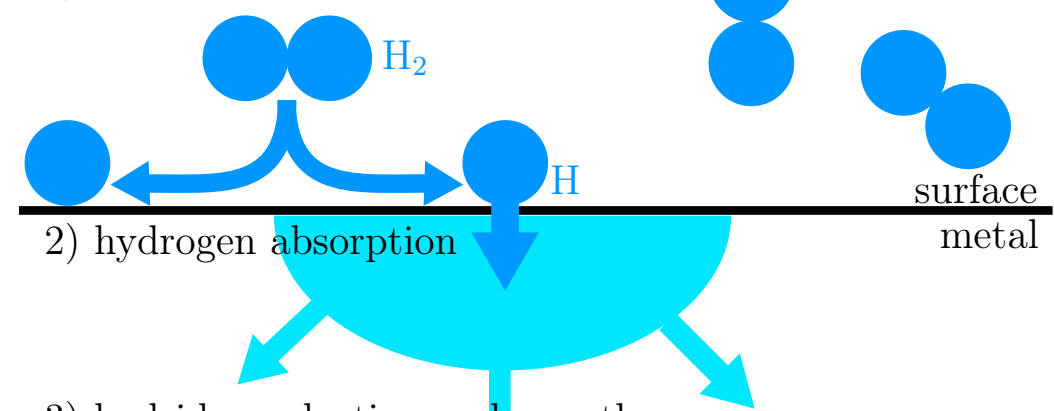

3) hydride nucleation and growth

In the following, each step will be discussed with regard to challenges limiting it in real $\mathrm{Mg}$ samples. It should be kept in mind that challenges in one step may also influence other steps. Hence, prohibiting influences discussed at one step could often also be attributed to another step. The general goal here is to give an overview of the 
challenges in the overall kinetic process of the $\mathrm{MgH}_{2}$ formation and what has been done in the past to improve this process. Improvements to the hydride formation kinetics can roughly be divided into two subtopics:

- Including additives (with adding hydrogen catalysts as a subtopic)

- Changing the microstructure

Hydrogen catalysts, in this work, only promote the dissociation of the hydrogen molecule into atomic hydrogen (and the reversed process). Overall, these measures may improve one of the steps given above, or several at once. Sometimes it is not clear where the main improvement happens. Furthermore, while additives have often shown catalytic effects [34, 35, 31], alloying with transition metals shows improved hydrogen storage properties because of thermodynamic changes. A prominent example is $\mathrm{Mg}_{2} \mathrm{Ni}$ [35]. While a lot of work on alloying $\mathrm{Mg}$ or $\mathrm{MgNi}_{2}$ with additional components has been done it is not of interest here, since the resulting improvements are not kinetics but thermodynamic changes (further information can be found in references [19] and [20]). However, this shows that it can be difficult to separate the influence of additives between thermodynamic or kinetic contributions.

\section{Challenges in step 1) Surface dissociation of hydrogen}

The first step for the formation of a hydride is the adsorption of hydrogen to the metal surface and the dissociation of the hydrogen molecule into two hydrogen atoms. Only then will the hydrogen atom dissolve into the metal [107]. For magnesium two things hinder the dissociation. First, magnesium forms an oxide layer in air [108, 109]. This will be discussed in regard to the second kinetic step of hydrogen absorption into the magnesium. Second the clean magnesium surface has a low dissociation probability for hydrogen [30, 31. This can be improved by adding catalysts to the magnesium, which dissociate the hydrogen more easily, but absorb it less easily than the magnesium. A prominent example is palladium $(\mathrm{Pd})$, which is also used in this work and will be discussed in detail below. Beforehand, some other examples from the literature will be highlighted.

Transition metals and their oxides have been found to improve the hydride formation kinetics and the effect has often been associated with hydrogen catalysis. Some of the most prominent examples is the work of Barkhordarian et al. who showed the catalytic effect of $\mathrm{NbO}_{5}$ [34, 110]. The catalyst was shown to improve de- and absorption of hydrogen from $\mathrm{MgH}_{2}$ powder, which was milled together with the catalyst. It was shown that the oxide acts as a catalyst, as opposed to the pure metal after reduction by the magnesium. Similar but smaller effects have been found for other transition metals and their oxides, such as vanadium, titanium, 
niobium, vanadiumoxide $\left(\mathrm{V}_{2} \mathrm{O}_{5}\right)$ and many more [19, 20, 21, 29, 50, 111, 47]. Pozzo and Alfè performed a systematic study of the influence of transition metals on the hydrogen dissociation at Mg surfaces [112. They found that the influence depends on the lowering of the dissociation barrier on one side, and on the binding between the hydrogen atom and the transition metal on the other side. An optimal effect was found for $\mathrm{Ni}, \mathrm{Rh}, \mathrm{Cr}$ and $\mathrm{Pd}$. While a catalytic effect is often assumed, it is not always explicitly confirmed. Therefore, another kinetic or even thermodynamic effect cannot be excluded.

Iron was added in many thin films studied in this work to study its influence on the grain boundary diffusion 1 . Magnesium and iron can be assumed to be immiscible at room temperature. Nayeb-Hashemi et al. give a maximal solubility of iron in magnesium of 0.0041 at $\%$ at $649^{\circ} \mathrm{C}$ [69]. Therefore, in this work a complete demixing of the two materials is assumed ${ }^{2}$. The iron may then segregate along the grain boundaries of the magnesium and change their structure and diffusion properties. Furthermore, iron is known to catalyse hydrogen uptake into magnesium [113, 114, 115]. At the same time it does not take up any relevant amount of hydrogen [116]. At higher pressures and temperatures magnesium, iron and hydrogen can form $\mathrm{Mg}_{2} \mathrm{FeH}_{6}$ [117, 118]. The phase was first presented by Didisheim et al., who prepared it at a temperature of $500^{\circ} \mathrm{C}$ and a pressure of $6 \mathrm{MPa}$ [117. De Lima et al. showed that $\mathrm{Mg}_{2} \mathrm{FeH}_{6}$ can be prepared at lower temperatures and pressures after hot extrusion of $\mathrm{Mg}-\mathrm{Fe}$ at $200^{\circ} \mathrm{C}$ to $300^{\circ} \mathrm{C}[119,120,121$. However, because hydrogen loading was done at room temperature in this work, no formation of $\mathrm{Mg}_{2} \mathrm{FeH}_{6}$ is expected.

Palladium is of unique interest for this work because it caps all magnesium thin films studied in this work. The $\mathrm{Pd}$ capping layer is supposed to improve the hydrogen catalysis and prevent surface oxidation of the magnesium [36, 37, 38]. Figure 2.4 (a) shows the bulk phase diagram of the palladium-hydrogen system. The formation and desorption enthalpy change for the bulk system was found to be equal, within measuring accuracy. A value of $\Delta H_{P d}^{d e s}=\Delta H_{P d}^{a b s}=-19.1 \mathrm{~kJ}(\mathrm{molH})^{-1}$ is reported for both [122]. The formation and desorption entropy change was found to be about $\Delta S_{P d}^{d e s}=\Delta S_{P d}^{a b s}=-46.3 \mathrm{JK}^{-1}(\mathrm{molH})^{-1}[122]$. This leads to a formation pressure of palladiumhydride of about $1000 \mathrm{~Pa}$ at $293 \mathrm{~K}$, about three orders of magnitude larger than the formation pressure of magnesiumhydride. As discussed above it is expected that the loading pressure in the thin film layer is higher than the bulk value, because of stress influences.

Palladium capping layers have been used before on magnesium and magnesium alloy thin films. Intermixing of the two metals has been reported in these cases [30, 37, 124, 125]. Krozer and Kasemo reported intermixing/alloying starting at

\footnotetext{
${ }^{1}$ The iron contains some chromium and traces of other elements.

${ }^{2}$ Because of this complete demixing of the two materials a phase diagram is not given in this work, as it does not offer further insight.
} 


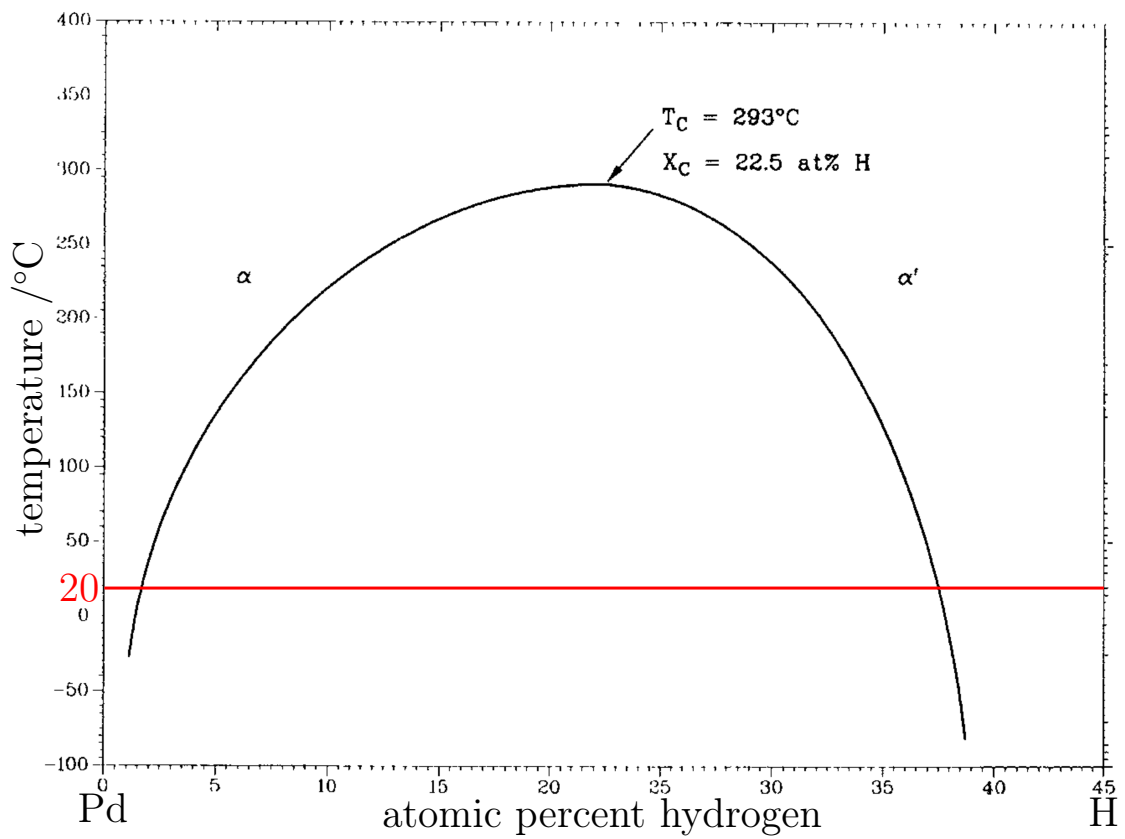

(a) Phase diagram of the palladium-hydrogen system according to Manchester et al. [122. The thin films in this work were loaded at $20^{\circ} \mathrm{C}$ as indicated by a red line.

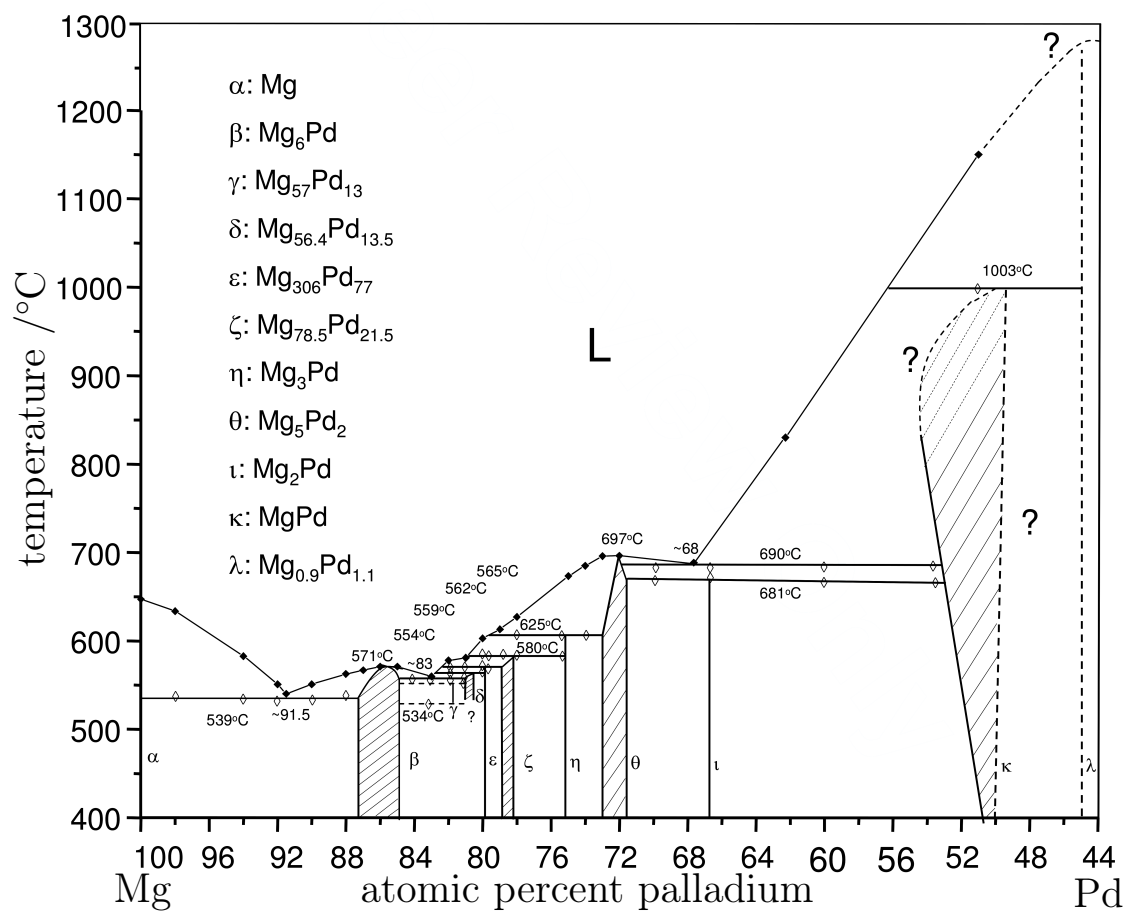

(b) Phase diagram of the magnesium-palladium system according to Makongo et al. [123.

Figure 2.4.: Phase diagrams of the palladium-hydrogen and palladium-magnesium system. 
temperatures of $390 \mathrm{~K}$ [30]. Eijt et al. sputtered thin $\mathrm{Mg}$ films and found formation of a $\mathrm{Mg}_{2} \mathrm{Pd}$ interlayer after hydrogen loading at temperature of $480 \mathrm{~K}$ and higher [125]. It can not be excluded that the interlayer already forms before loading and the high temperature is not necessary. In accordance with this idea, Singh et al. reported the formation of a mixed layer for pulsed laser deposited thin films at room temperature [37. The layer was identified as $\mathrm{MgPd}$ and was reported to be already present after production of the films. Furthermore, Slack et al. found an interdiffusion of palladium and magnesium for thin films during hydrogen loading at room temperature [124. They proposed the formation of $\mathrm{Mg}_{5} \mathrm{Pd}_{2}$ and $\mathrm{Mg}_{6} \mathrm{Pd}$. Overall, the formation of an intermixing layer between the $\mathrm{Mg}$ thin films and $\mathrm{Pd}$ capping layer is to be expected for high temperatures and may happen at room temperature. However, it does not prevent $\mathrm{MgH}_{2}$ formation. The main effect found was a decrease in gravimetric hydrogen density, since some magnesium is lost for the formation of magnesiumdihydride when it forms the magnesium-palladium interlayer. It is notable that all groups proposed different $\mathrm{Mg}-\mathrm{Pd}$-phases for their interlayer. Figure 2.4 (b) shows the Mg-Pd-phase diagram [123]. One can see that several phases can form down to room temperature (one can also compare the phase diagram at room temperature given by A. A. Nayeb-Hashemi and J. B. Clark [126]). Which phase forms in the interlayer may therefore strongly depend on the conditions during film deposition.

In this work some $\mathrm{Pd}$ capping layers contain iron (all magnesium thin films with iron additives have also similar additive concentrations in the palladium). Zhang et al. found that this increases the plateau pressure compared to pure $\mathrm{Pd}$ [127]. For iron contents of over $10 \mathrm{at} \%$ they found no formation of hydrides. However, this does not mean that the $\mathrm{Pd}$ capping layers do not catalyze hydrogen, if they contain iron. Klose et al. prepared Pd capping layers in the same sputter system utilized for this work, working at similar iron contents [128]. They were still able to load thin niobium films under the palladium-iron capping layer verifying that hydrogen is still dissociated by the palladium-iron capping layer.

\section{Challenges in step 2) Hydrogen absorption into the magnesium}

After the hydrogen molecule has been dissociated in its atomic form it needs to enter the magnesium (see figure 2.3. . For pure magnesium in air this is prohibited by a magnesiumoxide layer on top [32, 33]. The layer has been studied by different groups, often using X-ray exited photoelectron spectroscopy (XPS) [108, 109, 129, 130, 131. For oxygen or dry air atmospheres a thin $\mathrm{MgO}$ layer forms on the surface with thicknesses of $(2.5 \pm 1.0) \mathrm{nm}$ in the first $15 \mathrm{~min}$ [108, 131]. In atmospheres with water content the $\mathrm{MgO}$ starts to form an additional $\mathrm{Mg}(\mathrm{OH})_{2}$ layer [108, 129, 131, 132. It seems to be this layer that continues to grow and corrode the underlying magnesium. 
This is not surprising as the proposed corrosion mechanism of magnesium is [133]:

$$
\mathrm{Mg}+2 \mathrm{H}_{2} \mathrm{O} \longrightarrow \mathrm{Mg}(\mathrm{OH})_{2}+\mathrm{H}_{2}
$$

Chen et al. gave a different interpretation of their XPS results. They associated the peak, otherwise always associated to $\mathrm{Mg}(\mathrm{OH})_{2}$, to oxygen in a "defective" chemical environment. "Defective" in their context means that the oxygen does not occupy its normal $\mathrm{MgO}$ lattice position.

Regarding the hydride formation the $\mathrm{MgO}$ layer seems to be prohibitive. Gonzales et al. studied the diffusion of hydrogen (and deuterium) in $\mathrm{MgO}$ and found no significant diffusion below $1750 \mathrm{~K}$ [134]. Vigeholm et al. showed that this will not always prevent hydride formation, since thick oxides will crack and thereafter allow hydrogen to reach pure magnesium regions [39]. Because of the volume expansion during the hydride formation (see section 2.1.2), this is especially true after the first hydride formation (as long as the pure magnesium has no contact with oxygen between the loading cycles). Another possibility of breaking the oxide layer is heating the sample in vacuum before hydrogen loading [40]. This results in the decomposition of the hydroxide layer at temperatures above $623 \mathrm{~K}$. Interestingly, Hjort et al. showed improved kinetics in the case of magnesiumoxides present between thin films of magnesium and palladium [135]. They explained this by an increased nucleation probability of the hydride at the oxides. This agrees with the fact that they found improved kinetics, but a lower total hydrogen content.

\section{Challenges in step 3) Nucleation and growth of the hydride phase}

The final kinetic step of the hydrogen uptake is the nucleation of the Mg hydride phase and growth (see figure 2.3). This step is generally agreed on as the limiting parameter of the hydride formation [41, 42]. The hydrogen release on the other side was often found to be limited by a surface process, possibly the hydrogen desorption from the surface [42, 136]. In opposition, Tanniru et al. found the nucleation of the magnesium $\alpha$-phase as the limiting process for the hydrogen release [137]. It should be kept in mind that the limiting process depends on the overall system and it may change, depending on catalysts, additives, surrounding atmosphere and much more. Because of this, the limiting process is evaluated separately from the diffusion measurements in this work (for the theoretical basis see section 2.3).

A model for the hydride nucleation in magnesium was given by Vigeholm et al. [46]. He showed that the hydride formation in magnesium particles is nucleation and growth dependent. In addition, he found a pressure dependence on the nucleation process. This was confirmed and explained further by Uchida et al. (see figure 2.5) [44, 76]. He proposed a model in which the hydride nucleates on the surface of the magnesium. The nuclei density is pressure dependent, because it is dependent 

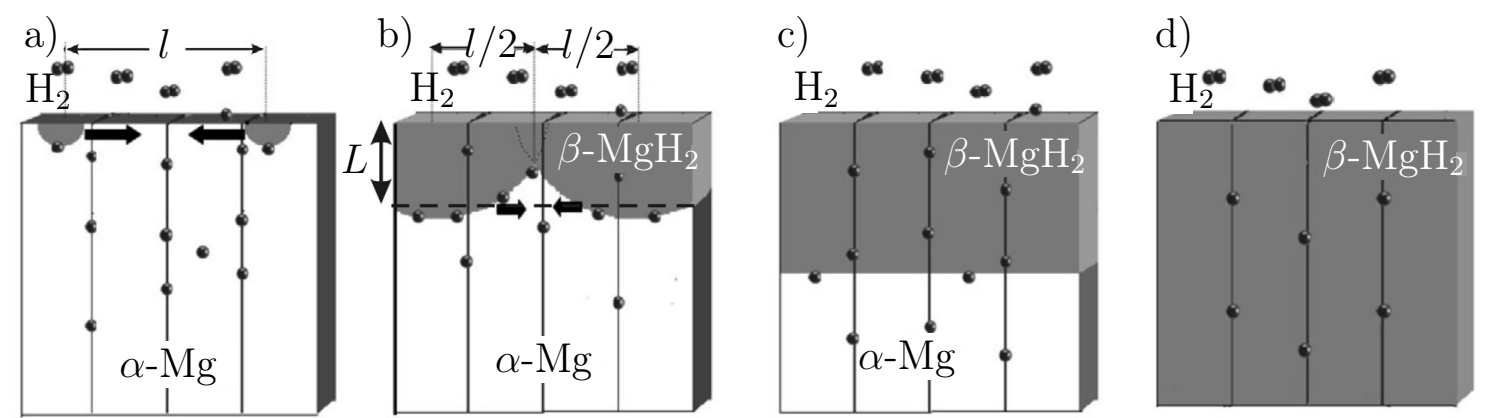

Figure 2.5.: Schematic drawing of the hydride nucleation and growth model as published by Uchida et al. [44]. a): The hydride phase $\left(\beta-\mathrm{MgH}_{2}\right.$, gray), nucleates at the film surface with a mean distance of $l$. It grows half-spherical into the $\alpha-\mathrm{Mg}$ phase (white). Fast diffusion through the a-Mg phase is possible until neighboring semi-spheres meet each other (b). Afterwards, a magnesiumhydride blocking layer of thickness $L$ forms. c) and d): Further hydride formation is limited by the flux along the grain boundaries

on the driving force. Higher driving forces lead to more nuclei. In the case of gas loading, a higher driving force means higher hydrogen gas pressures above the plateau pressure. Furthermore, more nuclei lead to a thinner closed hydride layer on the top of the magnesium sample. As will be discussed below, the hydrogen diffusion in magnesiumdihydride is low, therefore the hydrogenation process slows down significantly once a closed hydride layer is formed.

The so called blocking layer (a magnesiumdihydride layer preventing further hydrogenation) has been discussed in the literature [24, 43] and Uchida's model explains well several experimental results found. For example, it makes clear that decreasing grain and particle sizes have not only the advantages of smaller diffusion paths, as often discussed [19, 20, 47, 48, 49, 50. Smaller single particles will have a lower, absolute number of nuclei for the same driving force (being proportional to the same nuclei density). Hence, these nuclei can grow further into the magnesium before forming a closed hydride layer. The same argument holds for thinner magnesium film sizes.

After the blocking layer is formed magnesium often remains below the hydride (see figure $2.5 \mathrm{c}$ )). To reach the maximum gravimetric hydrogen density of $7.6 \mathrm{wt} \%$, this magnesium needs to be hydrided as well. For this, additional hydrogen needs to diffuse through the hydride layer to the hydride/magnesium interface. That hydrogen is the diffusing species in magnesiumdihydride is not trivial and was debated for some time. Mintz et al. and Sander proposed the magnesium cation as diffusing species [151, 144]. Later, Luz et al. showed that the $\mathrm{H}^{-}$anion is the diffusing species by means of a Kirkendall experiment [41]. The same result was reached by Hao and Scholl using DFT simulations [45].

In the following, diffusion coefficients for the system are collected from the litera- 


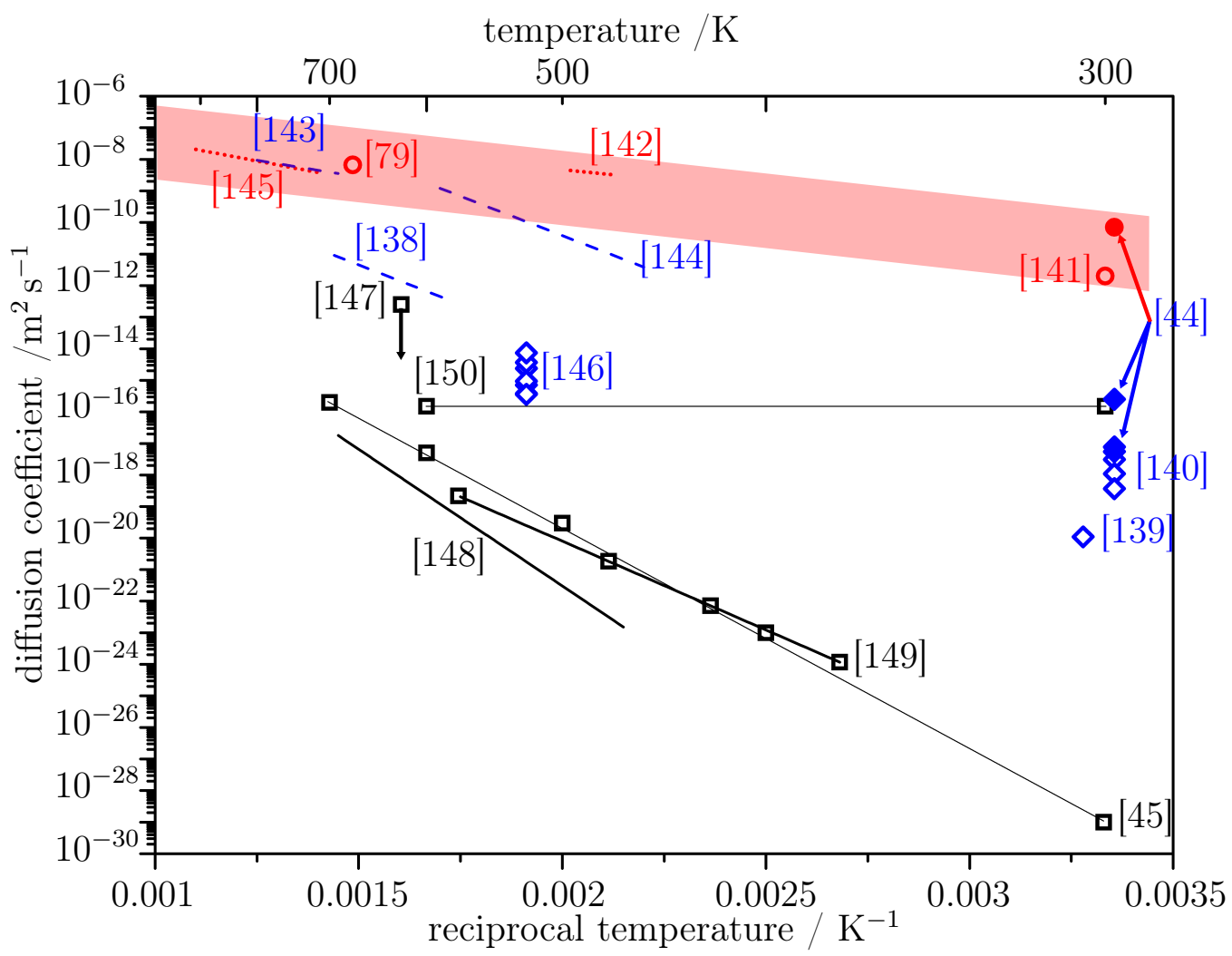

Figure 2.6.: Collection of literature data of the hydrogen diffusion coefficient in the magnesium-hydrogen system. Single diffusion coefficients, measured at a given temperature, are marked by points. Thick lines are plotted where activation energy and preexponential factor were measured. Thin lines connect measurements by a single group. The data is divided into diffusion in the $\beta$-phase (marked in black squares and solid lines), measurements during phase transformation (marked in blue diamonds and dashed lines) and in the $\alpha$-phase (marked in red circles and dotted lines). The values measured in the $\alpha$-phase fall in a corridor marked by a red box. For each literature value the responding reference is given.

ture. The diffusion coefficient directly shows how long it takes to transport hydrogen through a unit length of material (magnesium or magnesiumdihydride). More information will be given in the following section 2.2. The collected diffusion coefficients are presented in Figure 2.6, as a function of the reciprocal temperature $1 / T$. The data is divided into diffusion coefficients in the $\alpha$-phase (red circles and red dotted lines) and diffusion coefficients influenced by the $\mathrm{MgH}_{2} \beta$-phase. This includes measurements in pure magnesiumdihydride (black squares and solid black lines) and during the hydride formation or decomposition (blue diamonds and dashed blue lines). It should be noted that the y-scale covers 24 orders of magnitude.

The diffusion coefficients in the $\alpha$-phase cover a small corridor. The maximum variance is slightly higher than one and a half order of magnitude $\left(7 \cdot 10^{-11} \mathrm{~m}^{2} \mathrm{~s}^{-1}\right.$ measured by Uchida et al. [44] and $2 \cdot 10^{-12} \mathrm{~m}^{2} \mathrm{~s}^{-1}$ calculated by Vegge [141]). The 
value from Uchida et al. was measured at $293 \mathrm{~K}$ by permeation measurements [44. Nishimura et al. employed the same method at temperatures between $473 \mathrm{~K}$ to $493 \mathrm{~K}$ and came to very similar results [142. Expanding their results to $293 \mathrm{~K}$ gives a value of $6 \cdot 10^{-11} \mathrm{~m}^{2} \mathrm{~s}^{-1}$. Vegge utilized density functional theory (DFT) to calculate their value at $300 \mathrm{~K}$ [141]. Schimmel et al. employed ab initio dynamical simulations to calculate a diffusion coefficient of $6.6 \cdot 10^{-9} \mathrm{~m}^{2} \mathrm{~s}^{-1}$ at $673 \mathrm{~K}$ [79]. Extrapolation of the data of Nishimura et al. shows a value of $1.9 \cdot 10^{-8} \mathrm{~m}^{2} \mathrm{~s}^{-1}$ at the same temperature [142]. This value is twice as high as the value of Schimmel [79]. However, it should be kept in mind that extrapolating measurements to other temperatures easily leads to a large error, because of an exponential influence of any error in the activation energy ${ }^{3}$. Simensen measured the degassing of hydrogen from magnesium to extract the diffusion coefficient at temperatures from $723 \mathrm{~K}$ to $893 \mathrm{~K}$ [145]. Expanding their measurement to room temperature would result in a value of $3.3 \cdot 10^{-14} \mathrm{~m}^{2} \mathrm{~s}^{-1}$, much lower than the other measurements. However the expansion contains a large error because of the large temperature extrapolation. The measurement of Simensen [145] match very well to calculations of Schimmel et al. [79]. At $673 \mathrm{~K}$ the experiments of Simensen [145] predict a value of $2 \cdot 10^{-9} \mathrm{~m}^{2} \mathrm{~s}^{-1}$ about a factor of three lower than the calculations of Schimmel et al. [79]. Overall, the diffusion coefficients of hydrogen in the $\alpha$-phase are known from the literature over a large temperature region and are in good agreement, considering measurement inaccuracies. The corridor of diffusion coefficients in the $\alpha$-phase is marked in figure 2.6 with a red box.

The literature data of diffusion coefficients measured in $\mathrm{MgH}_{2}$ samples shows a large scatter. Therefore, the data has been divided further. Diffusion coefficients that were measured in the already formed hydride are marked in black squares and black solid lines [147, 148, 45, 149, 150. Measurements that were performed during the hydride formation or decomposition are marked by blue diamonds and blue dashed lines [44, 138, 139, 140, 143, 144, 146]. First, the measurements in already hydrided $\mathrm{MgH}_{2}$ will be discussed. Hao and Scholl utilized DFT calculations to compute the diffusion coefficient in $\mathrm{MgH}_{2}$ at different temperatures [45]. The values fit well to the results of Yao et al. [149], who combined calculations with experimental results. Both groups found values between $10^{-16} \mathrm{~m}^{2} \mathrm{~s}^{-1}$ at $700 \mathrm{~K}$ and $10^{-29} \mathrm{~m}^{2} \mathrm{~s}^{-1}$ at $300 \mathrm{~K}$. Corey et al. measured commercial coarse grained $\mathrm{MgH}_{2}$ by nuclear magnetic resonance (NMR) spectroscopy at temperatures between $500 \mathrm{~K}$ to $674 \mathrm{~K}$ [148]. They determined a hopping rate $\omega$ of $Z \nu \approx \omega=2.5 \cdot 10^{15} \mathrm{~s}^{-1}$ and an activation energy of $E=166 \mathrm{~kJ} \mathrm{~mol}^{-1}$. $Z$ is the number of surrounding jump sites and $\nu$ the vibrational mean frequency (see section 2.2.2 for further information). To compare those values with the diffusion coefficients by Yao et al. and Hao and Scholl [45, 149], a jump distance $s$ needs to be estimated. As a rough estimate of the jump distant, $s=5 \AA$ is in this work taken from the volume of the $\mathrm{MgH}_{2}$ unit cell $V_{M g H 2}^{\text {unit }}=4.51^{2} \cdot 3.01 \AA^{3} \approx 61.22 \AA^{3}$ (compare section 2.1.2). Inserting the

\footnotetext{
${ }^{3}$ see equations 2.13 and equation 2.15 for the influence of the activation energy on interstitial and substitutional diffusion coefficients.
} 
thus approximated jump distance $s$ into the equation $D=(1 / 6) s^{2} \omega \exp (-E / R T)$ 4 allows calculating the diffusion coefficient. Even with the rough estimate of the jump distant $s$, the data of Corey et al. [148] matches the values of Yao et al. and Hao and Scholl well [45, 149]. Ball-milled $\mathrm{MgH}_{2}$ samples, also measured by Corey et al., show a second, strongly sample-dependent contribution. This contribution was much faster and was especially dominant at low temperatures. No quantitative values are given for this fast component, but Corey et al. attribute it to contributions by grain boundaries [148]. Toepler et al. employed neutron scattering to measure the diffusion in $\mathrm{MgH}_{2}$ [147]. However, their value of $2.5 \cdot 10^{-13} \mathrm{~m}^{2} \mathrm{~s}^{-1}$ at $T=623 \mathrm{~K}$ is only a maximum value, as their measurement accuracy did not allow them to measure smaller values. This fact is indicated by an arrow in figure 2.6. Further hydrogen diffusion coefficients in magnesiumhydride were measured by Stioui et al. using NMR spectroscopy at temperatures between $300 \mathrm{~K}$ to $600 \mathrm{~K}$ [150]. They found no temperature dependence on their diffusion coefficient $D=1.5 \cdot 10^{-16} \mathrm{~m}^{2} \mathrm{~s}^{-1}$. This result seems to contradict the theoretical background of diffusion. As will be discussed further in section 2.2 a temperature dependence on the diffusion coefficient is always expected and to the knowledge of the author of this work the opposite has never been measured or proposed.

The final set of diffusion coefficients is measured during the hydride formation or decomposition are marked in blue. Renner and Grabke optically measured the penetration depth of the hydride in $\mathrm{Mg}-2 \mathrm{wt} \% \mathrm{Ce}$ samples [143]. They found a closed layer of hydride growing into the sample and diffusion constants similar to the data of Simensen measured in the $\alpha$-phase [145. However, because of the closed hydride layer it is not to be expected that Renner and Grabke measured diffusion in magnesium. The high values may originate from an additional influence of the cerium in the samples. Stander measured the diffusion coefficient gravimetrically [144]. He found that the hydrogenation is diffusion limited after a closed hydride layer is formed, fitting the model of Uchida. The measured diffusion coefficient is above $10^{-10} \mathrm{~m}^{2} \mathrm{~s}^{-1}$ at $576 \mathrm{~K}$. Extrapolating their value to room temperature gives a value of $\approx 10^{-18} \mathrm{~m}^{2} \mathrm{~s}^{-1}$. Cěrmák and Král applied gas volumetry desorption measurements to study the diffusion coefficient [138]. They chose their pressures to ensure the hydride decomposition is diffusion limited. Interestingly, they found an activation energy of the diffusion process similar to that of Renner and Grabke with $96 \mathrm{~kJ} \mathrm{~mol}^{-1}$ 143. In contrast, the pre-exponential factor, measured by Cĕrmák and Král is $1.5 \cdot 10^{-4} \mathrm{~m}^{2} \mathrm{~s}^{-1}$, which is half the value determined by Renner and Grabke [143]. Spatz measured a diffusion coefficient of $1.1 \cdot 10^{-20} \mathrm{~m}^{2} \mathrm{~s}^{-1}$ at $305 \mathrm{~K}$ [139]. As experimental procedure they studied XPS on a thin film of magnesium (no film thickness is given). Similar, Qu et al. and Uchida et al. measured the diffusion in thin films of thicknesses between $20 \mathrm{~nm}$ to $100 \mathrm{~nm}$ [140] and of $500 \mathrm{~nm}$ and $2800 \mathrm{~nm}$ 44]. Uchida et al. differentiated two diffusion regimes. The first was associated with

\footnotetext{
${ }^{4}$ compare equation 2.15 for the substitutional diffusion coefficient, $f \cdot \exp \left(\left(\Delta S+\Delta S_{V}\right) / R\right)$ is
} assumed as one for simplicity. 
the time before a closed hydride layer is formed, the second with the time after. The average of the diffusion coefficients for different film thicknesses in the first regime was given as $2.5 \cdot 10^{-16} \mathrm{~m}^{2} \mathrm{~s}^{-1}$. The diffusion coefficients for the second regime is on average $5.5 \cdot 10^{-18} \mathrm{~m}^{2} \mathrm{~s}^{-1}$. The values of $\mathrm{Qu}$ et al. fit well to this second value [140]. Their values increase with decreasing film thickness from $3.7 \cdot 10^{-19} \mathrm{~m}^{2} \mathrm{~s}^{-1}$ to $7.8 \cdot 10^{-18} \mathrm{~m}^{2} \mathrm{~s}^{-1}$. Bobet et al. measured diffusion coefficients from $\mathrm{Mg}$ particles (with additions of metal catalysts) by gas volumetry measurements [146]. Their diffusion coefficients vary between $3.8 \cdot 10^{-16} \mathrm{~m}^{2} \mathrm{~s}^{-1}$ and $7.4 \cdot 10^{-15} \mathrm{~m}^{2} \mathrm{~s}^{-1}$ at $523 \mathrm{~K}$. The variance in the values can not clearly be ascribed to the different catalysts used or the way these were introduced (ball milling or decoration from a supercritical fluid).

Overall, many hydrogen diffusion coefficients in the magnesium-hydrogen system have been measured over the years and only the values measured in the $\alpha$-phase resemble each other. As soon as hydrogen diffusion in the magnesiumdihydride affects the overall diffusion process a large scatter in the diffusion coefficient is observed in the literature. In accordance with the main question of this work this may be due to the influence of grain boundaries, which most of the time have not been taken into account.

\subsection{Diffusion}

This section will give a short introduction into diffusion and especially into grain boundary diffusion in solids. This should allow for a better understanding of the diffusion process and the importance of the diffusion constant as a key parameter. It will introduce the most important equations necessary to evaluate the diffusion measurements as described in chapter 3. First, Fick's first and second law will be introduced. These equations are the analytical foundation for all diffusion problems. Thereafter, a chapter differentiates between interstitial and substitutional diffusion, the two main processes for atom diffusion in solids. Afterwards, the most important model for grain boundary diffusion, as introduced by Fisher [58, will be discussed briefly. Finally, the grain boundary diffusion behavior in polycrystals is introduced, as originally discussed by Harrison [64].

\subsubsection{Fick's laws of diffusion}

The basic equations for any diffusion problem are known as Fick's first and second law, originally introduced by A. Fick in 1855 [152]. Further information can be 
found in textbooks, e.g. in [153] or [51]. The general concepts will be presented in the following.

The particle flux $J$ is defined as the concentration $c$ of particles times their velocity $v: J=c \cdot v$. The velocity itself is a product of the particles mobility $B$ and the driving force $F$ exerted on them. The driving force $F$ can be expressed as the negative gradient of a potential $P$. All of this allows the description of the flux in dependence on the driving potential in one dimension:

$$
J=-c B \frac{\partial P}{\partial x} .
$$

The potential for a chemical gradient is the chemical potential

$$
\mu=\mu_{0}+R T \ln (\gamma c)
$$

- $R$ is the gas constant, $T$ is the temperature, $\mu_{0}$ is the chemical potential under standard conditions and $\gamma=a / c$ is the activity coefficient, being the quotient of activity $a$ and the concentration of particles:

$$
J=-c B \frac{\partial \mu}{\partial x}=-B R T\left(1+\frac{\partial \ln \gamma}{\partial \ln c}\right) \frac{\partial c}{\partial x}=-D_{c h e m} \frac{\partial c}{\partial x},
$$

with the generally valid relationship

$$
\frac{\partial \mu}{\partial x}=\frac{\partial \mu}{\partial \ln c} \frac{\partial \ln c}{\partial c} \frac{\partial c}{\partial x}
$$

Equation 2.7 gives the flux as the negative product of the diffusion coefficient and the concentration gradient. This is the most well known form of Fick's first law. However, it should be remembered that the driving force in general is not the concentration $c$, but the chemical potential $\mu$. Therefore, diffusion also can take place if the concentration is spatially invariant but the chemical potential is not or vice versa.

Equation 2.7 also introduces the chemical diffusion coefficient $D_{\text {chem }}$. It can be described as the product of the self diffusion coefficient $D_{S}=B R T$ and the thermodynamic factor $W=1+\frac{\partial \ln \gamma}{\partial \ln c}$. For ideal solutions (or simply dilute solutions) $\gamma$ is constant and equation 2.7 becomes

$$
J=-B R T \frac{\partial c}{\partial x}=-D_{S} \frac{\partial c}{\partial x} .
$$

In this work $D$ abbreviates the chemical diffusion coefficient. If another diffusion coefficient is meant it will be labeled accordingly.

Fick's second law can be derived by combining Fick's first law (equation 2.7) with the continuity equation:

$$
\frac{\partial c}{\partial t}=-\frac{\partial J}{\partial x}
$$


This simply describes the concentration changes over time as the difference of inwards and outwards flux in a spatial element $d x$. Combining the continuity equation with Fick's first law leads to Fick's second law

$$
\frac{\partial c}{\partial t}=\frac{\partial}{\partial x}\left(D \frac{\partial c}{\partial x}\right)=D \frac{\partial^{2} c}{\partial x^{2}}
$$

The middle term is the general form of Fick's second law. However, if the diffusion coefficient is location independent the final term can be applied.

For completeness the more general three-dimensional form of Fick's first and second law are given:

$$
\begin{aligned}
J & =-c B \nabla \mu=-D \nabla c, \\
\frac{\mathrm{d} c}{\mathrm{~d} t} & =\nabla(D \nabla c)=D \Delta c .
\end{aligned}
$$

\subsubsection{Substitutional and interstitial diffusion}

The diffusion in solids can be differentiated by the mechanism through which an atom diffuses through the solid. Interstitial atoms diffuse by jumps from one interstitial lattice space to the next. As long as the solid solution is dilute enough the diffusion process of one atom is independent of the other atoms. This is known as interstitial diffusion. For high concentrations of the interstitial atom, as well as for self diffusion in a solid, a vacant site in the lattice is needed. Both cases are described by the substitutional diffusion, which differs from the interstitial diffusion in the need of vacant sites. In the following interstitial diffusion is meant as a short form for dilute interstitial diffusion.

Figure 2.7: Interstitial jump of atom $\mathrm{B}$ in a (2D) matrix of atoms of kind A. To jump distance $s$ the atom has to overcome the energy barrier $\Delta G$. The jump occurs statistically into one free interstitial site surrounding the atom.

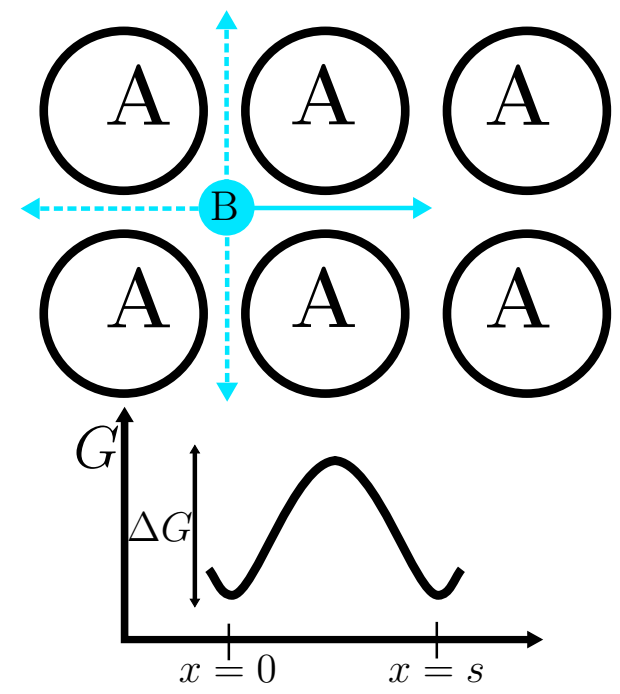


Interstitial diffusion is a simple statistical process. An atom randomly jumps from its lattice site into one of the surrounding sites, as shown in figure 2.7. Assuming a simple cubic lattice, this is described by the interstitial diffusion coefficient

$$
D_{\text {inter }}=\frac{1}{2 d} s^{2} \Gamma
$$

where $d$ is the dimension of the diffusion process, $s$ is the distance to the nextneighboring lattice sites or the jump distance in this case. Finally, $\Gamma$ is the jump frequency. It depends on the number of surrounding jump sites $Z$, the vibrational mean frequency $\nu$ and the energy barrier $\Delta G=\Delta H-T \Delta S$ separating two interstitial positions (see figure 2.7). This leads to the following form of the interstitial diffusion coefficient:

$$
D_{\text {inter }}=\frac{1}{2 d} s^{2} Z \nu \exp \left(\frac{\Delta S}{R}\right) \exp \left(-\frac{\Delta H}{R T}\right)=D_{0}^{i} \cdot \exp \left(-\frac{E_{A}^{i}}{R T}\right),
$$

where $D_{0}^{i}$ is the pre-exponential factor, which is temperature independent. $E_{A}^{i}$ is the activation energy of the interstitial diffusion process.

For substitutional diffusion self-diffusion has to be distinguished from the more complicated diffusion in binary (or higher) alloys. Self-diffusion differs from the interstitial diffusion discussed above in two points. First, jumps are no longer independent, it is more likely for an atom to jump back into the original site. Therefore a correlation factor $0 \leq f \leq 1$ is introduced:

$$
D_{s u b}=\frac{1}{2 d} s^{2} \Gamma \cdot f
$$

In addition, a neighboring vacancy is necessary for a jump to occur. The probability for such a vacancy $\chi_{V}=\exp \left(-\frac{\Delta G_{V}}{R T}\right)$ depends on its formation energy $\Delta G_{V}$ of it. Putting all of this together allows writing the substitutional diffusion coefficient in similar fashion to the interstitial diffusion coefficient:

$$
D_{s u b}=\frac{1}{2 d} s^{2} Z \nu f \exp \left(\frac{\Delta S+\Delta S_{V}}{R}\right) \exp \left(-\frac{\Delta H+\Delta H_{S}}{R T}\right)=D_{0}^{s} \cdot \exp \left(-\frac{E_{A}^{s}}{R T}\right) .
$$

Again, $D_{0}^{s}$ is the pre-exponential factor, which is temperature independent and $E_{A}^{s}$ is the activation energy of the substitutional diffusion process.

For a description of the the diffusion in binary alloys the lattice needs to be taken into account. Since substitutional diffusion utilizes a vacancy mechanism, vacancies move in the lattice. This is sketched in figure 2.8. For a net flux of atoms in one direction one has an equal flux of vacancies in the opposite direction. This becomes important in a binary alloy where both atomic species have different diffusion coefficients $D_{A / B}$. Because of the vacancy flux the lattice itself moves and it becomes helpful to view 
Figure 2.8: Substitutional diffusion in a binary alloy. For substitutional diffusion vacancies are necessary (indicated by dashed circle). The diffusion process can be described by the jump of the atom into a vacancy, as well as the jump of the vacancy through the lattice.

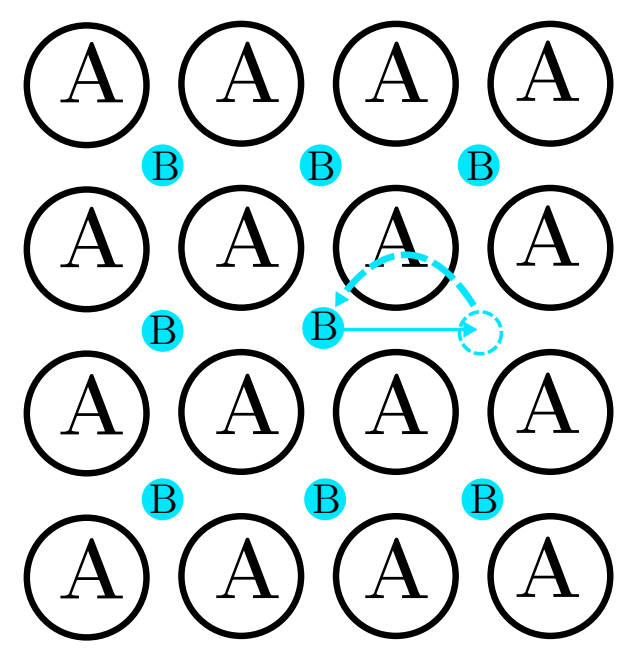

the flux not relative to the lattice but to a fixed position. The total flux $J^{T o t}$ through a stationary plane is the sum of the diffusion flux $J^{D}$ of one atomic species relative to the lattice and the flux of the lattice $J^{v}$ itself:

$$
J_{A / B}^{T o t}=J_{A / B}^{D}+J_{A / B}^{v}=-D_{A / B} \frac{\partial c_{A / B}}{\partial x}+v \cdot c_{A / B},
$$

where $c$ is again the concentration, here of a single atomic species. The overall concentration $c=c_{A}+c_{B}$ is constant in time and space, $v$ being the velocity of the lattice. Using the continuity equation 2.8 on the overall concentration $c$ gives:

$$
\frac{\partial c}{\partial t}=\frac{\partial c_{A}}{\partial t}+\frac{\partial c_{B}}{\partial t}=-\nabla J_{A}-\nabla J_{B} \stackrel{!}{=} 0
$$

The last equation notes the fact that the overall concentration is time independent. This allows determining the lattice velocity $v$ in dependence on the movement of the two atomic species:

$$
v=\frac{1}{c}\left(D_{A}-D_{B}\right) \nabla c_{A}=\frac{1}{c}\left(D_{B}-D_{A}\right) \nabla c_{B} .
$$

Using the continuity equation 2.8 and the equation for the total flux 2.16 of one atomic species an equation comparable to Fick's second law can be derived:

$$
\frac{\partial c_{A / B}}{\partial t}=\nabla\left(\left(\frac{c_{A} D_{B}+c_{B} D_{A}}{c}\right) \nabla c_{A / B}\right)=\nabla\left(\tilde{D} \nabla c_{A / B}\right) .
$$

Equation 2.17 and

$$
\tilde{D}=\frac{c_{A} D_{B}+c_{B} D_{A}}{c}
$$

are known as Darken's equations, after L.S. Darken who published them in 1948 [154. 
The diffusion in the $\mathrm{Mg} \alpha$-phase can be described by interstitial diffusion (see equation 2.13), because of the low hydrogen concentrations (see chapter 2.1.1). However, because the the $\mathrm{MgH}_{2} \beta$-phase is a stoichiometric phase, the diffusion of $\mathrm{H}$ in $\mathrm{MgH}_{2}$ should be described by substitutional diffusion in a binary alloy (see equation 2.15 and equation 2.19).

\subsubsection{Grain boundary diffusion}

The overall diffusion in solids is strongly influenced by defects like dislocations, grain boundaries or simply surfaces. Often, especially for substitutional diffusion, these defects enhance the overall diffusivity [51]. However, the opposite can be the case, as found for hydrogen in steel [52, 53, 54, 55]. Oudriss et al. showed for hydrogen diffusion in polycrystalline nickel that for a single sample both cases can act simultaneously. On the one side, grain boundaries accelerate diffusion and on the other side trapping effects decelerate it [56, 57]. In this work only nanocrystalline samples are studied, therefore grain boundaries should be the dominant species of defects. Because of this, the most important models of grain boundary diffusion will be introduced in the following.

In 1951, J.C. Fisher introduced a simple model for diffusion in an isolated grain boundary [58]. He assumed the grain boundary to be a slab of material inserted into the bulk (see figure 2.9 for details). The diffusion is assumed to be in the direction of the grain boundary, which shall be parallel to the z-direction and perpendicular to the y-direction. With a diffusion coefficient $D_{G B}$ of the grain boundary and $D_{V}$ of the grains Fick's second law becomes

$$
\frac{\partial c_{V}}{\partial t}=D_{V}\left[\frac{\partial^{2} c_{V}}{\partial y^{2}}+\frac{\partial^{2} c_{V}}{\partial z^{2}}\right]
$$

for the diffusion in the volume and

$$
\frac{\partial c_{G B}}{\partial t}=D_{G B}\left[\frac{\partial^{2} c_{G B}}{\partial y^{2}}+\frac{\partial^{2} c_{G B}}{\partial z^{2}}\right]
$$

for the diffusion in the grain boundary. Taking into account continuity of concentration and continuity of flux at the interface between grain boundary and bulk, the two diffusion equations can be combined into one single equation:

$$
\frac{\partial c_{V}}{\partial t}=D_{G B} \frac{\partial^{2} c_{V}}{\partial z^{2}}+\frac{2 D_{V}}{\delta} \frac{\partial^{2} c_{V}}{\partial y^{2}},
$$

where $\delta$ is the width of the grain boundary, which is assumed to be small enough that there are no concentration variations along it. 


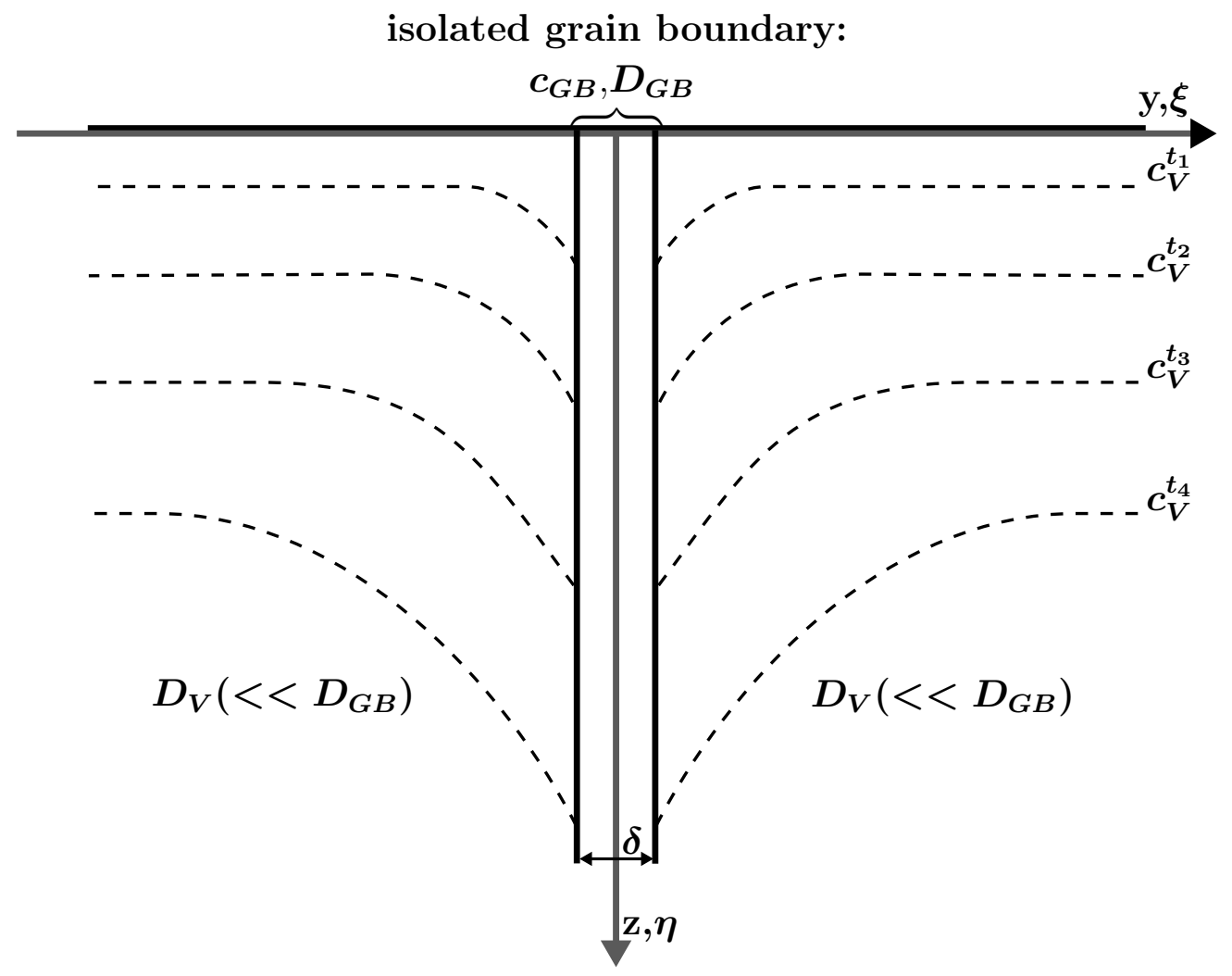

Figure 2.9.: Two-dimensional sketch of Fisher's model for an isolated grain boundary [58. The grain boundary has width $\delta$, the grain boundary diffusion coefficient is $D_{G B}$ and a uniform concentration $c_{G B}$. The bulk concentration profile $c_{V}$ is indicated for four times with $t_{1}<t_{2}<t_{3}<t_{4}$. In this model it is always assumed that $D_{G B}>>D_{V}$

The exact solution of this equation was given in 1954 by Whipple [59], assuming a constant source:

$$
\begin{aligned}
c_{V} & =c_{1}+c_{2} \\
& =c_{0} \cdot \operatorname{erfc}(\eta / 2)+\frac{c_{0} \eta}{2 \pi^{1 / 2}} \int_{1}^{\Delta} \frac{\exp \left(-\eta^{2} / 4 \sigma\right)}{\sigma^{3 / 2}} \operatorname{erfc}\left(\frac{1}{2}\left(\frac{\Delta-1}{\Delta-\sigma}\right)^{1 / 2}\left(\xi+\frac{\sigma-1}{\beta}\right)\right) \mathrm{d} \sigma,
\end{aligned}
$$

where $c_{0}$ is the concentration of the constant source. The overall concentration in the grain $c_{V}$ is the sum of the concentration transported by volume diffusion $c_{1}$ and the concentration transported in the grain boundary $c_{2} . \eta=z /\left(D_{V} t\right)^{1 / 2}$ and $\xi=$ $(y-\delta / 2) /\left(D_{V} t\right)^{1 / 2}$ are dimensionless variables for the $\mathrm{z}$ - and $\mathrm{y}$-direction respectively. $\xi$ is defined as zero inside the grain boundary. $\beta=((\Delta-1) \delta) /\left(2\left(D_{V} t\right)^{1 / 2}\right) \approx$ $\left(\delta D_{G B}\right) /\left(2 D^{3 / 2} t^{1 / 2}\right)$ determines the relationship of the bulk diffusion in the $\mathrm{y}$ - and z-direction and $\Delta=D_{G B} / D_{V}$ the ratio of the two diffusion constants.

While this solution is analytically exact, it could not be applied to experimental data. It was mainly the work of Levine and MacCallum [60], as well as Le Claire 
61] which made it possible to apply the analytic solutions to actual experimental data. They assumed that $D_{G B}>>D_{V}$, which simplifies the analytic solution as $\Delta \rightarrow \infty$, while $\beta$ stays finite. By averaging the concentration in the y-direction and looking at areas far enough away from the source, $\overline{c_{2}}>>\overline{c_{1}}$ can be assumed. Levine and MacCallum did show that a plot of $\bar{c}$ as function of $z^{6 / 5}$ was linear. In addition to numerical integration of the simplified integrals in Whipple's solution, this allowed to extract the grain boundary diffusion coefficient by a relatively simple equation (for $\beta>10$ ):

$$
\delta D_{G B}=2\left(\frac{D_{V}}{t}\right)^{1 / 2}\left(-\frac{\partial \ln \bar{c}}{\partial z^{6 / 5}}\right)^{-5 / 3} 0.78^{5 / 3} .
$$

Gryaznov et al. proposed an improved version of LeClaire's form in equation 2.21 [155]. Instead of fitting a straight line to a $\ln \bar{c}$ vs $z^{6 / 5}$ plot and using its slope, $(\partial \ln \bar{c}) /\left(\partial z^{6 / 5}\right)$ should be plotted over the time. Afterwards, the maximum of this plot should be found and applied instead of the slope. Also, instead of the factor $0.78,0.77+0.71 \alpha$ with $\alpha=\delta / 2\left(D_{V} t\right)^{-1 / 2}$ should be used.

In 1961 Suzouka gave the analytical solution for an instantaneous source and applied it a few years later to experiments, using a similar procedure as Levine and MacCallum and Le Claire [62, 63]. He found the same linearity of $\bar{c}$ versus $z^{6 / 5}$ and the same equation 2.21, but with different multiplicative factors. Table 2.2 gives the multiplication factors in dependence on $\beta$. For a given $\beta$ the correct factor is simply substituted for $0.78^{5 / 3}$ in equation 2.21

\begin{tabular}{cl}
$\beta$ & factor \\
\hline$\beta<100$ & $0.70^{5 / 3}$ \\
$100<\beta<1000$ & $0.74^{5 / 3}$ \\
$1000<\beta$ & $0.775^{5 / 3}$
\end{tabular}

Table 2.2: Multiplicative factors for the Suzouka solution of diffusion in an isolated grain boundary from an instantaneous source. The factors are given in dependence on $\beta=((\Delta-$ 1) $\delta) /\left(2\left(D_{V} t\right)^{1 / 2}\right)$.

\section{Segregation}

For impurity diffusion or diffusion in alloys segregation becomes important. Segregation is the enrichment of atoms (or in some cases molecules) at boundaries or defects [156]. In this work, segregation always implies grain boundary segregation, meaning the excess of one atomic species at grain boundaries. Segregation is described by the segregation factor:

$$
s(T)=\frac{c_{G B}}{c_{V}} .
$$

The segregation factor $s$ mainly has a geometric influence on grain boundary diffusion. The grain boundary width, as relevant for the diffusion process, is not only the 
true geometric width $\delta$ but the product of the geometric width and the segregation factor $s$. This means that for the diffusion process the grain boundary may be wider or thinner depending on the segregation. A result is that equation 2.21 and the corresponding solution for the instantaneous source changes to [51]

$$
s \delta D_{G B}=2\left(\frac{D_{V}}{t}\right)^{1 / 2}\left(-\frac{\partial \ln \bar{c}}{\partial z^{6 / 5}}\right)^{-5 / 3} 0.78^{5 / 3}
$$

\section{Atomistic models and orientation dependencies}

Up to here the grain boundary was treated as an undefined slab with a different diffusion coefficient than the bulk. How this diffusion coefficient is established and how it depends on the surrounding bulk are questions which have drawn interest in the last decades. Relevant questions are: How does the grain boundary orientation relative to the diffusion direction influence the diffusion, how does the diffusion depend on the type of grain boundary and what is the atomistic model for diffusion in grain boundaries. Answering these questions has proven difficult, as it is challenging to experimentally study these problems. Here, a short overview of some answers that have been established will be given.

Establishing an atomistic model of the movement of particles in grain boundaries is difficult to do experimentally. Up until now, most successful were numerical methods, especially molecular dynamics (MD) simulations and Monte-Carlo simulations [66]. An important result was that the correlation factor $f$ is of high importance in grain boundaries [65, 67]. It was found that the correlation factor can be strongly anisotropic and temperature dependent. Suzuki and Mishin published an overview of the atomistic mechanism of grain boundary diffusion, especially differentiating between low and high temperature [157]. Because of the temperature range studied in this work only the low temperature results are presented. It was found that for self-diffusion in grain boundaries, vacancies and interstitials exist as equal partners. There are also several mechanisms for the movement of both defects. Overall this fits well to the diffusion mechanisms in metallic glasses. Additional results are:

- A significant anisotropy of the diffusion coefficient exists, regarding the orientation of the grain boundary to the diffusion direction.

- The grain boundary structure influences the diffusion coefficient by orders of magnitude

Both results have been confirmed by other groups in different experiments, as discussed in the following. 
The influence of the diffusion direction relative to the grain boundary orientation can be measured in bicrystals. In addition, bicrystals allow the study of different kinds of grain boundaries. However, the production of high quality bicrystals with well defined grain boundaries is difficult. Nevertheless, several groups have worked on bicrystals and some general trends were found. Diffusion is faster along the tilt axis of a grain boundary than along any other direction [51, 158, 159, 68]. The effect is strongest for low angle grain boundaries, where it can be explained by a dislocation model. However, the effect is also present for high angle grain boundaries. A temperature dependency exists and the difference of diffusion coefficients for different diffusion directions decreases with increasing temperatures [51, 68].

Additionally, a dependence on the grain boundary diffusion coefficient on the coincidence site lattice (CSL) has been discussed in the literature. The CSL is formed by the fraction of lattice sites which are shared by the two lattices of grains forming the grain boundary. The reciprocal density of coincidence sites compared with the total number of sites is labeled $\Sigma[51,160$. A low sigma therefore means a high number of coincidence sites. It has often been found that grain boundary diffusion shows minima at orientations with low $\Sigma$ [161, 162, 163]. However, Ma and Balluffi found no such minima in their research of grain boundary diffusion [164, 165]. They explained it by the fact that other groups did not measure $D_{G B}$ directly but the triple product $s \delta D_{G B}$ (see equation 2.23). Therefore, Ma and Balluffi attributed the minima to the influence of segregation and not to the grain boundary diffusion coefficient. However, they measured at relatively low number of tilt angles compared with other groups. Because of this, they may have missed the minima in their measurements. An indication for the existence of diffusion coefficient minima in dependence on the grain boundary tilt angle was given by Han et al. [166]. They improved the classical structural unit model (SUM) to calculate the grain boundary energy. The SUM describes the structure of grain boundaries as a combination of so called structural units (SU) [167, 168, 169]. Grain boundaries made up of a single SU are called delimiting. Further, grain boundaries with a tilt angle between the tilt angles of two delimiting grain boundaries are combinations of the SUs of the delimiting grain boundaries. This simple model has been helpful for the description of grain boundaries and was e.g. applied by Ma and Balluffi to interpret their findings [164, 165]. Han et al. updated the classical SUM by taking into account that several metastable grain boundary structures exist, which offer a lower energy state for some ranges of tilt angles. They applied their metastable structural unit model (MSUM) to calculate the grain boundary energies of [001] and [111] symmetric tilt boundaries. Their results show minima in the grain boundary energy. These energy minima probably correspond to diffusion coefficient minima [170, 171]. For hydrogen Oudriss et al. showed two contradicting effects [56, 57]. On the one side, hydrogen is trapped in grain boundaries with a small $\Sigma$. On the other side, they found that triple points (the interface where three grains meet) increase the overall diffusion.

As shown above, the grain boundary diffusion in a single grain boundary is al- 
ready difficult to describe and depends strongly on the kind of grain boundary, its orientation relative to the diffusion direction and more. This makes diffusion in polycrystals challenging to model. Therefore, one has to make the assumption that the grain boundary diffusion for a polycrystal can be described by one single grain boundary diffusion coefficient. This is the simplification employed in the next section. However, in reality the different grain boundaries normally present in a polycrystal should be taken into account. This may for example be done by using a distribution of grain boundary diffusion coefficients around a median $D_{G B}$. Furthermore, to study the influence of changes in the grain boundary structure on the grain boundary diffusion coefficient $D_{G B}$ iron is added to the samples studied in this work. As described in chapter 2.1.4 iron does not solve in magnesium and may therefore segregate at the grain boundaries, possibly changing the grain boundary structure.

\subsubsection{Diffusion in polycrystals}

The diffusion of hydrogen in the samples studied in this work are strongly influenced by grain boundaries. However, since they are polycrystals they are no isolated grain boundary. Therefore, the classical Fisher model does not apply. Diffusion in polycrystalls was first discussed by L.G. Harrison 64. He introduced three regimes (A, B and $\mathrm{C}$ ), which have since been called Harrison regimes. In the following they will be introduced briefly and their difference will be explained. Later on, the Harrison regimes have been divided further. Here, mainly the division of regime $\mathrm{B}$ into regime $\mathrm{B}_{1}$ to $\mathrm{B}_{4}$ will be considered, as introduced by Mishin and Razumovskii [172]. Finally it will be discussed how the grain size influences which regime will be reached under which conditions.

A graphical overview of all three Harrison regimes is given in figure 2.10. The three Harrison regimes can be separated by time $t$, grain size $d$ and the ratio of the diffusion coefficients of grain boundary and volume $\Delta=D_{G B} / D_{V}$. In most cases this ratio $\Delta$ is unknown. In a given measurement all regimes can be reached by changing several parameters such as time, temperature and grain size. For example at a fixed position $z$ in the sample, regime $\mathrm{C}$ is valid for short times, and the profile transforms via regime B into regime A for longer times [51, 66].

Regime A In regime A the GB diffusion and volume diffusion are similarly fast so that the diffusing species forms an isotropic diffusion front in the material (see figure 2.10). This leads to an effective diffusion coefficient $D_{\text {eff }}$ [51]:

$$
D_{e f f}=\frac{s \phi_{G B} D_{G B}+\left(1-\phi_{G B}\right) D_{V}}{1-\phi_{G B}+s \phi_{G B}} .
$$




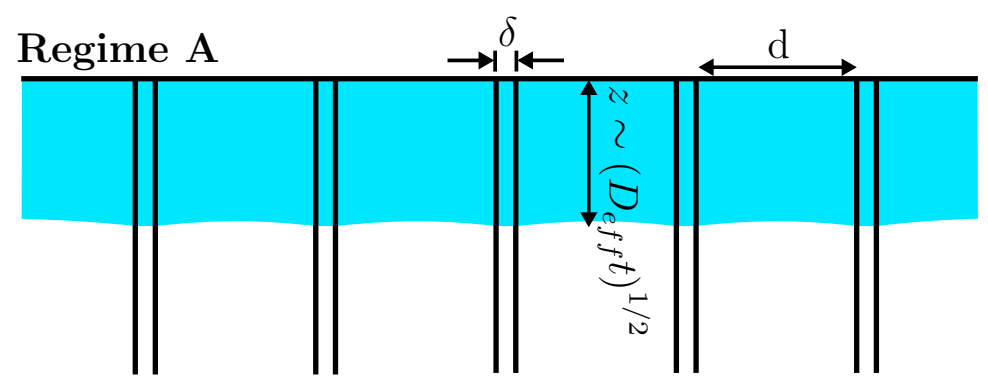

\section{Regime B}

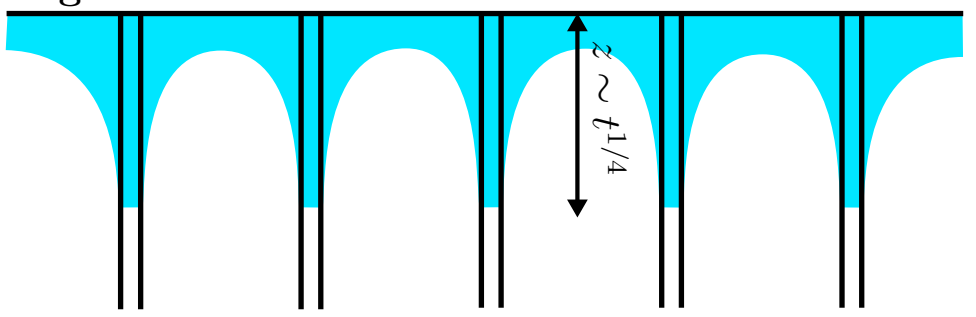

Regime C

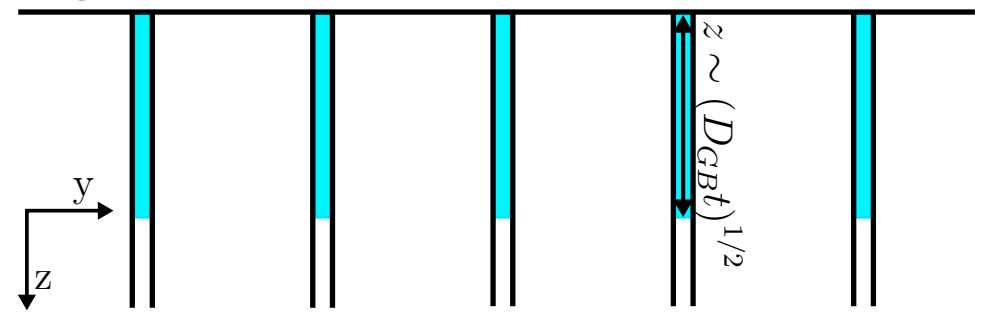

Figure 2.10: Visualization of the three Harrison regimes. Regime A shows an isotropic front which depends on the effective diffusion coefficient $D_{\text {eff }}$. Regime B can be described by the solutions for isolated grain boundaries (see chapter 2.2.3). Regime $\mathrm{C}$ is completely dominated by the grain boundary diffusion and can be described by an isotropic front which depends on the grain boundary diffusion coefficient $D_{G B}$.

The effective diffusion coefficient is the geometric average of the grain boundary and grain diffusion coefficients. $\phi_{G B}$ is the volume fraction of the GB, while $s$ is the segregation factor discussed above. Equation 2.24 is valid for columnar grains. For cubic grains, Belova and Murch found [173]:

$$
D_{e f f}=\frac{s D_{G B}\left(\psi(1-\psi) s D_{G B}+\left(1-\psi+\psi^{2}\right) D_{V}\right)}{\left(1-\phi_{G B}+s \phi_{G B}\right)\left(\psi D_{V}+(1-\psi) s D_{G B}\right)} .
$$

Here, $\psi=\psi_{y}=\psi_{z}$ is the grain boundary area fraction in $y$ - and $z$-direction. It can be shown that $D_{\text {eff }}$ from equation 2.25 is always smaller than from equation 2.24 . This originates from grain boundary diffusion perpendicular to the main diffusion in the $z$-direction.

Regime B In regime B, the diffusion is dominated by the grain boundary with outwards diffusion into the grain. The spacing of the grain boundaries is large in comparison with the volume reached by diffusion in the measurement time window. Hence, the boundaries can be treated as isolated. Therefore, Fisher's model (discussed in section 2.2.3 gives the analytic solution to this problem (see equation 
2.23 .

Regime C For regime C the grain diffusion is so low that all transport occurs in the grain boundaries. The result is again an isotropic front, however only within the grain boundaries. Therefore, the grain boundary diffusion coefficient $D_{G B}$ is also the diffusion coefficient of the whole system. This means that the measurement directly gives the grain boundary diffusion coefficient. Furthermore, this is the only regime where grain boundary segregation does not need to be considered, since the actual size of the grain boundary region does not influence the diffusion process. The diffusion process takes place in the grain boundary so more grain boundaries lead to more transport, but not faster or slower transport. Mishin and Razmunovskii studied the diffusion in moving grain boundaries with a constant velocity $v$ [174. They found that the movement does not change the diffusion speed in regime $\mathrm{C}$. This is not true for Regime $\mathrm{B}$, where the penetration depth is lower for a moving grain boundary [174]. Deng et al. studied the diffusion in Regime C for more than one type of grain boundary [175]. They studied how grain boundaries with fast and slow diffusion lead to an effective diffusion coefficient of the overall grain boundary network (the effective diffusion coefficient should not be confused with $D_{\text {eff }}$ in Regime A). No dependency on the grain size was found. They determined that the effective diffusion depends on the percolation of the fast diffusion grain boundaries. Before the fast diffusion grain boundaries form a closed network, the overall diffusion is governed by the slow diffusion grain boundaries. After a closed network is formed the effective diffusion of the overall system becomes similar to the discussion above of Harrison regimes. Two regions with different diffusion coefficients interact and the overall diffusion depends on the volume fractions of the regions and the ratio of the diffusion coefficients of the regions.

Regime $B_{1}$, Regime $B_{2}$, Regime $B_{3}$ and Regime $B_{4}$ Mishin and Razmumovskii further divided Harrison regimes [172]. They based this on the two dimensionless coordinates $\beta=\left(\delta D_{G B}\right) /\left(2 D^{3 / 2} t^{1 / 2}\right)$ (see section 2.2.3) and $\alpha=\delta /\left(2(D t)^{1 / 2}\right)$. $\beta$, as said above, describes the relationship of the $y$ - and $z$-direction of the bulk diffusion [61]. For example, if $\beta>>1$ bulk diffusion occurs mainly in the $y$-direction and no relevant transport is found in the $z$-direction. This means that transport in the z-direction can only happen by the grain boundary. $\alpha$ gives information about the relationship between diffusion along the grain boundary and leakage into the bulk. For $\alpha>>1$ leakage can be ignored, while for $\alpha<<1$ the grain boundary diffusion becomes quasi steady and leakage dominant. This ignores grain boundary segregation, which can be included by exchanging the geometric width $\delta$ by $s \delta$ as discussed above. 
The two coordinates are not independent:

$$
\frac{\alpha}{\beta}=\frac{D_{V}}{D_{G B}}<<1 .
$$

The inequality comes from the fact that $D_{G B}>>D_{V}$ is assumed. For the inequality to be true neither $\alpha \approx 1$ and $\beta \approx 1$ nor $\alpha>>1$ and $\beta<<1$ can be true. This leaves five different cases which correspond to regime $\mathrm{C}$ and four additional cases, which were labeled regimes $\mathrm{B}_{1}$ to $\mathrm{B}_{4}$ by Mishin and Razmumovskii. Harrison regime $\mathrm{A}$ is missing, because an isolated grain boundary is classified by these different regimes. However, all six regimes can be discussed in dependence on the grain size $d$.

Regime $\mathrm{C}$ is defined by no relevant leakage from the grain boundary and dominant grain boundary diffusion. This can be described in terms of $\alpha>>1$ and $\beta>>1$. For the regime $\mathrm{B}_{1}, \alpha \approx 1$ and leakage occurs into the bulk from the grain boundary. This increases in Regime $\mathrm{B}_{2}$ as $\alpha<<1$. This means that the grain boundary diffusion starts to become quasi-steady. However, as $\beta>>1$ still stands, the volume diffusion still applies predominantly in y-direction. This changes for regime $\mathrm{B}_{3}$ and $\beta \approx 1$. Finally, in regime $\mathrm{B}_{4}, \beta<<1$ and the bulk diffusion in the z-direction becomes dominant. This change from regime $\mathrm{C}$ through regimes $\mathrm{B}_{1}$ to $\mathrm{B}_{4}$ happens with increasing time at a fixed temperature or vice versa. However, because the time dependence scales with the square root of time, in real experiments, it is in generally impossible to reach all regimes at a fixed temperature.

The regimes in dependence on the grain size Not all the regimes discussed above will be reached for a given sample. The dependence on the regimes on the grain size will therefore be discussed in the following. To simplify the problem the regimes $B_{1}$ and $B_{3}$ will not be further discussed as they are transition states between regime $\mathrm{C}, \mathrm{B}_{2}$ and $\mathrm{B}_{4}$. Additional information can be found in "Fundamentals of grain and interphase boundary diffusion" by Kaur et al. [51.

A polycrystal with grain size $d$ can be classified by its grain size in relation to two characteristic diffusion lengths. The two lengths are characteristic of diffusion in the bulk $L=\left(D_{V} t\right)^{1 / 2}$ and in the grain boundary $L_{G B}^{C}=\left(D_{G B} t\right)^{1 / 2}$. However, $L_{G B}^{C}$ is only characteristic for the diffusion in regime $\mathrm{C}$, since it ignores the leakage from the grain boundary into the grain. For regime $\mathrm{B}_{2}$ one can define $L_{G B}^{B}=$ $\left(\left(\delta D_{G B}\right)^{1 / 2} t^{1 / 4}\right) /\left(4 D_{V}\right)^{1 / 4}$, which takes the leakage into account. $L_{G B}^{C}$ is always much larger than $L$. However, the same is not true for $L_{G B}^{B}$ because it grows slower in time than the other two length scales. Therefore $L_{G B}^{B}$ intersects $L_{G B}^{C}$ and $L$ at times $t^{\prime}=\left(s^{2} \delta^{2}\right) /\left(4 D_{V}\right)$ and $t^{\prime \prime}=\left(s \delta D_{G B}\right)^{2} /\left(4 D_{V}^{3}\right)$ respectively. Two corresponding sizes can be defined:

$$
L^{\prime}=\frac{s \delta}{2}\left(\frac{D_{G B}}{D_{V}}\right)^{1 / 2}
$$


and

$$
L^{\prime \prime}=\frac{s \delta}{2}\left(\frac{D_{G B}}{D_{V}}\right)
$$

Using these sizes polycrystals can be divided into three classes: coarse grained $\left(L^{\prime}<<L^{\prime \prime}<<d\right)$, fine grained $\left(L^{\prime}<<d<<L^{\prime \prime}\right)$ and ultra-fine grained $(d<<$ $\left.L^{\prime}<<L^{\prime \prime}\right)$. The advantage of this separation is that it can be defined for all times (and temperatures), which is not the case if e.g. $d$ is compared with $L$ and $L_{G B}$. The three classes and their sequence of regimes are collected in table 2.3 .

\begin{tabular}{c|c|c}
\hline class of polycrystals & relationship of length scales & sequence of regimes \\
\hline \hline coarse grained & $L^{\prime}<<L^{\prime \prime}<<d$ & $\mathrm{C} \rightarrow \mathrm{B}_{2} \rightarrow \mathrm{B}_{4} \rightarrow \mathrm{A}$ \\
fine grained & $L^{\prime}<<d<<L^{\prime \prime}$ & $\mathrm{C} \rightarrow \mathrm{B}_{2} \rightarrow \mathrm{B}_{2} \rightarrow \mathrm{A}^{\prime}$ \\
ultra-fine grained & $d<<L^{\prime}<<L^{\prime \prime}$ & $\mathrm{C} \rightarrow \mathrm{C}^{\prime} \rightarrow \mathrm{B}_{2} \rightarrow \mathrm{A}^{\prime}$ \\
\hline
\end{tabular}

Table 2.3.: Classes of polycrystals as divided by Kaur et al. [51]. The sequence of the regimes follows increasing time and does not include regime $\mathrm{B}_{1}$ and $\mathrm{B}_{3}$

For coarse grained polycrystals, one starts in regime $\mathrm{C}$ and goes to the different regimes $B$ with increasing time. The only difference from the previous discussed is that $L$ grows large enough that it reaches $d$. This marks the transition from regime $\mathrm{B}_{4}$ to regime $\mathrm{A}$, and the initially isolated grain boundaries start to interact. As said before the bulk diffusion becomes dominant in regime $\mathrm{B}_{4}$. Because the grain boundaries are still isolated in regime $\mathrm{B}_{4}$ they have no relevant influence on the overall diffusion. Therefore the diffusion is fully described by the grain diffusion coefficient $D_{V}$ and not by an average as in Harrisons regime A. No information about the grain boundary diffusion can be extracted in regime $\mathrm{B}_{4}$.

Fine grained polycrystals start in regime $\mathrm{C}$ and go into regime $\mathrm{B}_{2}$ for longer times (or higher temperature). However, the grain boundary length scale $L_{G B}^{B}$ becomes larger than the grain size $d$ before regime $\mathrm{B}_{4}$ would be reached. Therefore the grain boundaries are no longer isolated. However, this is not equivalent to regime $\mathrm{A}$, as the bulk diffusion length $L\left(<<L_{G B}^{B}\right)$ is still smaller than the grain size. This transition regime is labeled regime $\mathrm{B}_{2}{ }_{2}$. For calculation purposes regime $\mathrm{B}_{2}{ }_{2}$ can be treated like regime $\mathrm{B}_{2}$ (which means Harrison regime $\mathrm{B}$ as presented above). However, the boundary width $\delta$ has to be exchanged with an effective width $\delta^{\text {eff }}=\phi_{G B} / V$. $\phi_{G B}$ is the volume fraction of the grain boundary of volume $V$. Depending on the geometry, the difference between $\delta$ and $\delta^{e f f}$ is only a factor on the order of unity. After regime $\mathrm{B}_{2}$, regime $\mathrm{A}^{\prime}$ is reached. Regime $\mathrm{A}^{\prime}$ is identical to regime $\mathrm{A}$ from the diffusion process point of view, however, it can be shown that $d<<L^{\prime \prime}$ induces $D_{\text {eff }} \approx q s \delta D_{G B} / d$ (for further information see [51]). $q$ is a geometric factor depending on the grain shape.

Ultra-fine grained polycrystals go from regime $\mathrm{C}$ into a new regime $\mathrm{C}$ '. In regime C' the diffusant penetrates deep into the grain boundary without any considerable 
leakage. Similar to regime B' ${ }_{2}$ the process can be mathematically treated like regime C. The only difference is that the surface fraction of the grain boundaries has to be exchanged by the grain boundary volume fraction. From regime C' the diffusion process goes into regime $\mathrm{B}_{2}{ }_{2}$ and finally into regime $\mathrm{A}$ '.

Figure 2.11 shows for which grain size and $\Delta=D_{G B} / D_{V}$ which of the three different classes of polycrystals are reached. The lines dividing the classes are calculated from equations 2.27 and 2.28, $s \delta$ is assumed to be $1 \mathrm{~nm}$ as typical sizes of grain boundaries [176, 177].

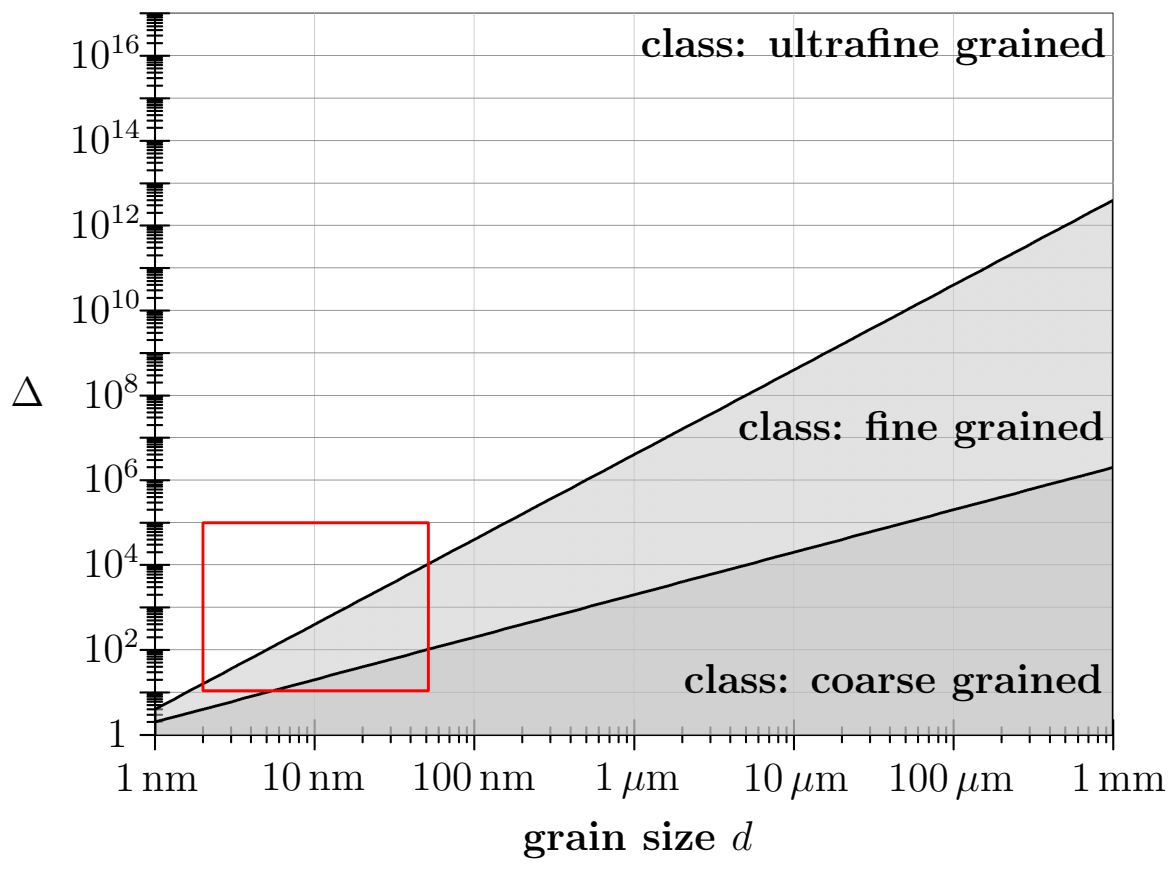

Figure 2.11.: Overview of the classes of polycrystals in dependence on the grain size $d$ and $\Delta=D_{G B} / D_{V}$. The dividing lines are calculated from $L^{\prime}$ and $L^{\prime \prime}$, assuming a grain boundary width of $1 \mathrm{~nm}$. The red box is the region that was analyzed here for the $\mathrm{Mg}-\mathrm{H}$ system by finite-element simulations using COMSOL Multiphysics (see chapter 3.6).

\subsection{Kinetic models for solid state transformations}

Section 2.1.4 introduced the different steps necessary to transform magnesium in a hydrogen atmosphere to a solid solution of hydrogen in magnesium and further to magnesiumdihydride (schematically shown in figure 2.3). The goal of this work is to measure the diffusion of hydrogen in the magnesium-hydrogen system and to determine the influence of grain boundaries on it. However, the diffusion is only one part of the overall kinetics. Therefore, it is only possible to isolate the diffusion if either 
the hydrogen movement in the system can be measured directly or if the diffusion dominates over other kinetic processes and any change in the system therefore depends on it. The methods applied in this work do not allow to microscopically plot the hydrogen movement (for the applied experimental methods and setups see chapter 3). They only allow to evaluate changes of different physical parameters during the uptake of hydrogen in the magnesium and the phase transformation to $\mathrm{MgH}_{2}$. Therefore, it is of interest to evaluate which kinetic process dominates the overall uptake and transformation. This is an old problem, which was famously discussed by Avrami [178, 179, 180, Johnson and Mehl [181] and Kolmogorov [182, as well as by many others later on. One way to determine the limiting kinetic process uses the following general procedure: One determines the transformed volume fraction $\phi$ or the process rate $\frac{\mathrm{d} \phi}{\mathrm{d} t}$ and describes it by a reaction model $f(\phi)=1 / k \frac{\mathrm{d} \phi}{\mathrm{d} t}$ or its integrated form $g(\phi)=k t$. $k=A \exp \left(-E_{A} / R T\right)$ includes the temperature dependence as well as the activation energy $E_{A}$ of the process and a pre-exponential factor $A$. In this work only the integrated form $g(\phi)$ of the reaction models will be utilized. In the following, some reaction models for the uptake of hydrogen into magnesium and the phase transformation to magnesiumdihydride will be briefly introduced. How these equations are applied to the measurements will be discussed in chapter 3.3.3. A general overview of important reaction models in both forms and their derivation was given by Khawam and Flanagan in 2006 [183. Another overview with a particular focus on thermal analysis data was given by Vyazovkin et al. [184]. Thermal analysis is a field were this approach is often applied and therefore much of the work comes from that research community. Finally, Pang and Li discussed the application of the procedure for metal-hydride systems [185]. Their proposed algorithm to evaluate the limiting kinetics is the basis for the algorithm utilized here and introduced in chapter 3.3. All these papers offer a deeper insight into the topic than can be included here and are good resources for additional information.

Johnson-Mehl-Avrami-Kolmogorov model (JMAK) This group of reaction models is named after the researchers that contributed to their development [178, 179, 180, 181, 182. They are generally applicable to a nucleation and growth process. However, they do not include influences of sur- or interfaces. The general form of the reaction model is often called "JMAK equation":

$$
(-\ln (1-\phi))^{1 / n}=k t .
$$

The exponent $n=d \cdot m+N$ gives additional information about the limiting process of the nucleation and growth [186, 187]. $d$ is the dimension of the process, meaning in which dimension a phase forms and grows. $m$ can have values of 1 if the growth is interphase controlled and $1 / 2$ if the growth is diffusion controlled. Finally, $N \geq 0$ gives information about the nucleation mode. For $N=0$ the nucleation is instantaneous (as in the case of preformed nuclei), while at $N=1$ the nucleation is continuous. Values higher or lower than one indicate de- and increasing 
nucleation. $n$ therefore goes from a value of 0.5 to 4 . However, some values can be interpreted by different combinations of $d, m$ and $N$. For example $n=1.5$ can result from diffusion limited growth in three dimensions for instantaneous nucleation $(3 \cdot 0.5+0=1.5)$ and from one-dimensional diffusion limited growth from constant nucleation $(1 \cdot 0.5+1=1.5)$. In such cases, the system under investigation needs to be taken into account.

The JMAK equation was developed under several assumptions:

- the sample is initially homogeneous,

- the product phase is randomly distributed,

- random nucleation (for the case of non-instantaneous nucleation),

- the average growth rate is independent of position,

- the reaction is not influenced by time-dependent processes.

Obviously, these assumptions will not be fulfilled at all times for every sample. This was discussed by Starink in detail [188]. He showed that some assumptions can be violated and the JMAK equation still stays valid. One example would be an inhomogeneous sample where all single areas transform with a constant $k$ and $n$. Under these conditions, the average of the sample still follows the JMAK equation. In general, it was concluded that if not all assumptions can be fulfilled, an alternative equation can be used:

$$
\phi=1-\left(1+\left(\frac{(k(T) t)^{n_{i}}}{\eta}\right)\right)^{\eta}
$$

where $n_{i}$ is similar to the JMAK exponent $n$ discussed above. $\eta \geq 1$ is the so called impingement parameter, which takes into account the impingement between the initially isolated areas of the newly formed phase. The impingement between growing regions is not included in the original JMAK equation. In this work the impingement parameter is employed to evaluate the validity of the JMAK equation. It can be shown that for $\eta \rightarrow \infty$ equation 2.30 is equivalent to equation 2.29 .

Contracting volume models (CV) These models assume rapid nucleation at the surface and afterwards, the transformation is controlled by the movement of the reaction interphase. In the two- and three-dimensional cases, this results in a contracting volume of the original phase towards the center of the geometry. The general equation for this model is given by [183]:

$$
1-(1-\phi)^{1 / d}=k t .
$$


$d$ is again the dimension of the kinetic process. For the one-dimensional case the equation simplifies to

$$
\phi=k t .
$$

This equation is the same as the one for a zero-order model, which describes the surface controlled case [189]. For metal-hydrogen systems this means that the kinetics are controlled by the hydrogen adsorption at the surface. This is an important point as it means that for a one-dimensional process one can not distinguish between a process that is limited by the surface versus the reaction interphase.

Diffusion models There are several different models based on the assumption that the reaction is limited by the diffusion of a reactant. In this work mainly Jander's model will be used, as introduced by W. Jander in 1927 [190]:

$$
\left(1-(1-\phi)^{1 / d}\right)^{2}=k t
$$

However, the Jander model assumes a constant interphase area of the reaction front. This is only valid for the one-dimensional case. Therefore, different models may be used if $d \neq 1$. One possibility would be the Ginstling-Brounshtein model (GB) 191:

$$
\begin{aligned}
(1-\phi) \ln (1-\phi)+\phi & =k t & \text { for } \mathrm{d}=2, \\
1-\frac{2}{3} \phi-(1-\phi)^{2 / 3} & =k t & \text { for } \mathrm{d}=3 .
\end{aligned}
$$

Figure 2.12 shows the models introduced above on a scale of arbitrary units for volume fraction $\phi$ and time. This allows a comparison of the curve shape of different models. Figure 2.12 (a) shows the contracting volume model (in black) and Jander's model (in blue) in one, two and three dimensions. In addition, The JMAK model was plotted for $n=0.6$ and $n=1.1$. It is apparent that the diffusion controlled models reach higher volume fractions faster than the CV models. This is also true for the Ginstling-Brounshtein model, which was omitted for clarity reasons. As Pang et al. noted, the JMAK model can be confused for the CV model if $n=1.1$ [185]. The same is true in the case of the diffusion controlled models and $n=0.6$. This is also shown in Figure 2.12 (a). While the two JMAK model curves have higher volume fraction values than the corresponding CV or Jander's model, their overall shape are similar. This is important to keep in mind for an evaluation of reaction models. Especially, if the JMAK equation (equation 2.29) is applied and $n$ evaluated. A value of $n=0.6$ or 1.1 can mean that the JMAK model is not reasonable and a CV model or diffusion based model should be applied.

Figure 2.12 (b) shows the JMAK model for different values of $n$ and the onedimensional CV model for comparison. Values of $n$ corresponding to interphase controlled growth are marked in black, while values corresponding to diffusion controlled 
growth are marked in blue. The curves for $n=1$ and $n=2$ have therefore been plotted in blue and black, since these values can correspond to both depending on the dimensionality of the process and the nucleation type. This illustrates the general problem that an evaluated $n$ can be interpreted differently so that additional information about the process need to be taken into account. Otherwise the JMAK model shows an increasingly sigmoidal shape for increasing values of $n$. For low values of $n$ the shape is deccelatory and similar to the CV or Jander model, as discussed above. 


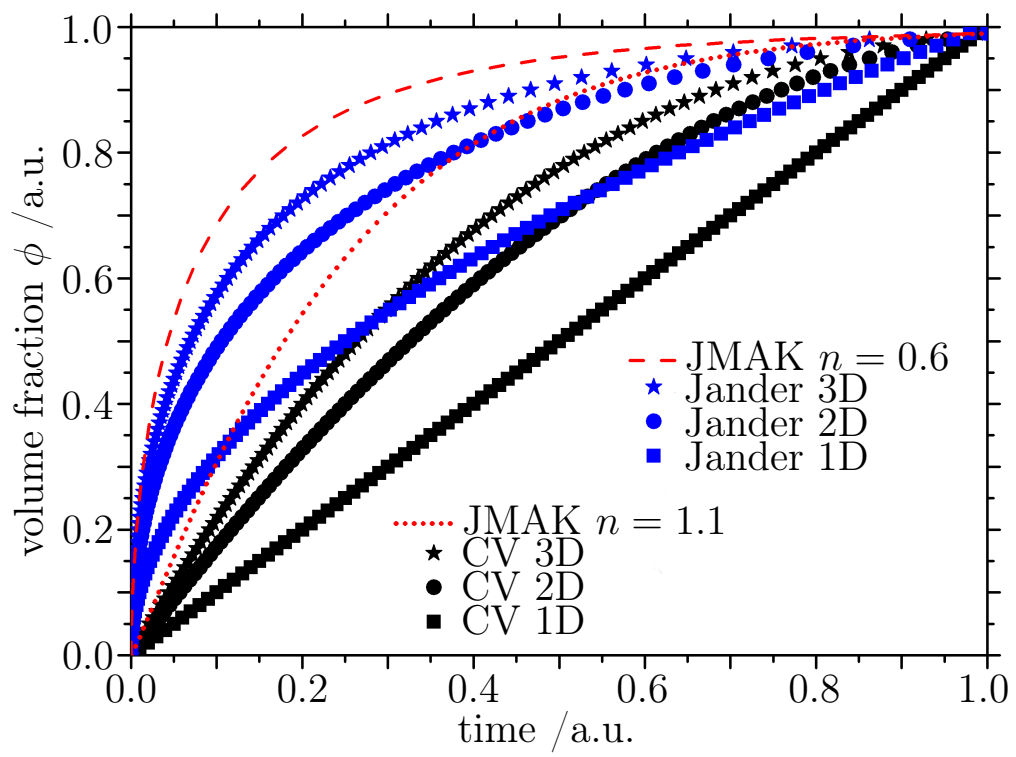

(a) Comparision of CV model and Jander's model in 1 to 3 dimensions

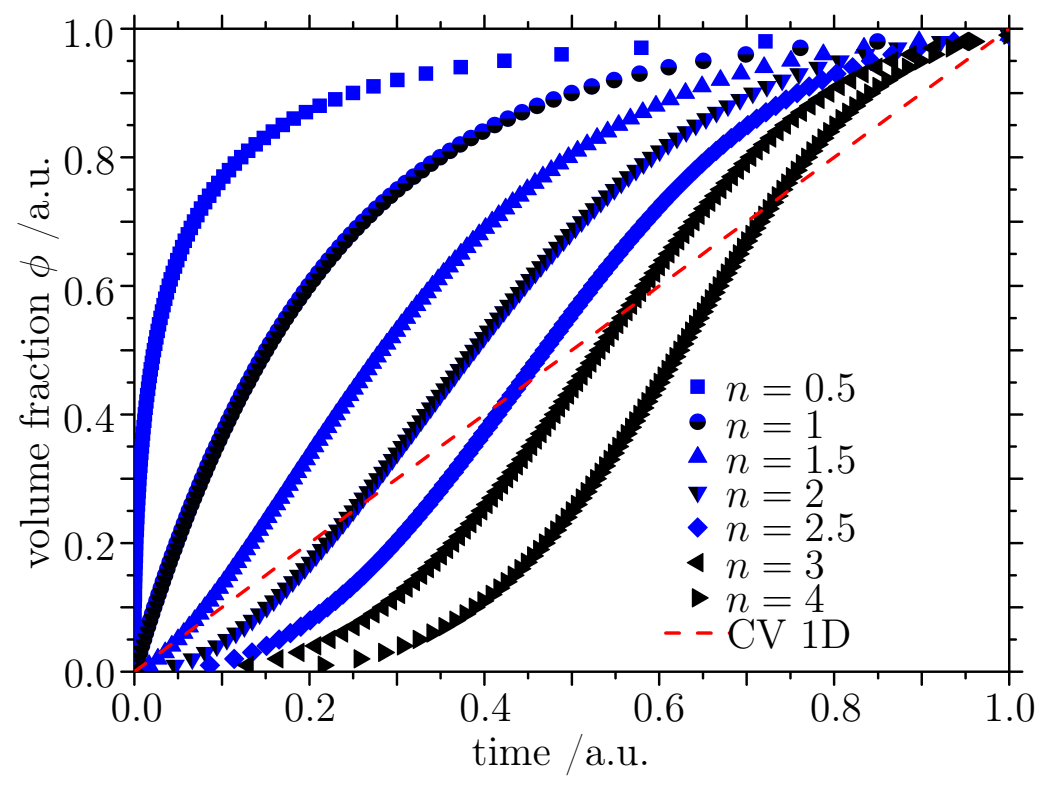

(b) Comparison of JMAK plots for different values of $n$

Figure 2.12.: Comparison of different reaction models in arbitrary units of time and reacted volume $\phi$. Figure (a) compares contracting volume models (CV) (black) with the diffusion model of Jander (blue) in one, two and three dimensions. In addition the Johnson-Mehl-Avrami-Kolmogorov model (JMAK) is plotted for a value of $n=0.6$ and $n=1.1$. Figure (b) compares the JMAK model for different values of $n$. Curves in black are interphase controlled, while blue curves are diffusion controlled. For values of $n=1$ and $n=2$ both are possible. The CV model in 1D is plotted to make the comparison with figure (a) easier. 


\section{Applied experimental methods and developed algorithms}

The following chapter presents the setups and instruments used, as well as algorithms developed to evaluate the measured data. First, the methods utilized to prepare thin $\mathrm{Mg}$ films are introduced (section 3.1). These techniques are, on the one side ultra-high vacuum (UHV) argon-ion-beam sputter (IBS) deposition and, on the other side, pulsed laser deposition (PLD). The samples prepared in these systems were measured in a gas volumetry chamber with in situ four-point resistance measurement. These two methods and the setups are discussed in section 3.2 and 3.3 respectively. The fourth section, 3.4 presents the electron microscopy applied to analyze the samples before, during and after hydrogen loading. The main focus of this chapter is the transmission electron microscopes (TEM) utilized to analyze the grain structure. In addition, a scanning electron microscope (SEM) was employed to verify the surface quality and for applying energy dispersive X-ray spectroscopy (EDX). The experimental setups used for X-ray diffractometry (XRD) are shown in section 3.5. This includes two synchrotron sources, PETRA P08 at the Deutsches Elektronen-Synchtrotron (DESY), Hamburg and Beamline BM20 at the European Synchrotron Radiation Facility (ESRF), Grenoble. The final section 3.6 discusses finite-element simulations that were performed using the software COMSOL Multiphysics ${ }^{\circledR}$, version 5.2.

\subsection{Sample preparation}

Two kinds of thin films were studied in this work: argon-ion beam sputtered films and pulsed laser deposited films. The two different techniques were utilized to obtain different grain sizes and grain morphologies in the Mg thin films. Sputter deposition of $\mathrm{Mg}$ thin films has been applied before by Uchida et al. and Qu et al. [44, 192]. They both found a fiber texture, with grain sizes in the out-of-plane direction being almost equal to the film thickness. In general, PLD samples can be prepared with smaller grain sizes and a more equiaxed shape as shown for other metal thin films [193, 194]. 
All samples were prepared on (100) silicon substrates of size $1 \mathrm{~cm}^{2}$ and thickness $0.5 \mathrm{~mm}$ (Crystec $\mathrm{GmbH}$ ). The miscut of the substrates is below $0.1^{\circ}$.

\subsubsection{Argon-ion beam sputtering}

The argon-ion beam sputter source was implemented in an ultra-high vacuum (UHV) apparatus (BESTEC) with a background pressure better than $10^{-7} \mathrm{~Pa}$. A prechamber allowed to insert the substrates and remove the prepared samples without breaking the UHV condition. The background pressure of the prechamber was always better than $10^{-5} \mathrm{~Pa}$ before substrates or samples are transfered. The sputter pressure was $4 \cdot 10^{-2} \mathrm{~Pa}$ and the argon gas had a purity of $99.9999 \%$. Up to four targets can be mounted on a rotational holder, allowing sputtering of different materials without removing the sample or target. The magnesium target had a purity of $99.9 \%$ and the average deposition rate was about $0.8 \mathrm{~nm} / \mathrm{s}$. All samples were deposited at $298 \mathrm{~K}$. Beforehand the target was cleaned for $0.5 \mathrm{~h}$ by pre-sputtering to remove surface oxides. Mg films of thicknesses ranging from $400 \mathrm{~nm}$ to $700 \mathrm{~nm}$ were prepared in this way. To prevent oxidation and to improve the hydrogen uptake a thin palladium layer was added on top of the magnesium, also at $298 \mathrm{~K}$ (compare the discussion in chapter 2.1.4). The palladium target had a purity of $99.9 \%$ and the average deposition rate is $1.1 \mathrm{~nm} / \mathrm{s}$. The Pd top layer was always between $20 \mathrm{~nm}$ to $40 \mathrm{~nm}$. Overall, the preparation follows the work of H. Uchida, who already prepared $\mathrm{Mg}$ thin films with the same system (for further information see [76]).

Some samples studied in this work contain steel additives (especially iron and chromium). They were prepared by changing the alignment of the ion source, resulting in the deposition of steel additional to the target material. The additive content in each sample is noted in table 4.1, containing an overview over all samples. The additive content was measured by energy dispersive X-Ray (EDX) spectroscopy (see section 3.4). The EDX system employed in this work allows quantifying of additives down to a weight percentage of about $1 \mathrm{wt} \%$. The resolution is exact to about $1 \mathrm{wt} \%$ for the thin layers discussed in this work. Because of this, samples that are labeled as additive free may still have up to $1 \mathrm{wt} \%$ of additive, which the EDX system is not able to quantify.

\subsubsection{Pulsed laser deposition}

The pulsed laser deposited samples were prepared by F. Schlenkrich and F. Döring under supervision of H.-U. Krebs and by S. Hoffmann-Urlaub. The Mg films were deposited using a $\mathrm{KrF}$ excimer laser (wavelength of $248 \mathrm{~nm}$, pulse duration of 30 
ns, repetition rate of $10 \mathrm{~Hz}$ ) [195]. The films were grown at room temperature in ultrahigh vacuum of $10^{-6} \mathrm{~Pa}$ using a commercial $\mathrm{Mg}$ target of a purity of $99.9 \%$.

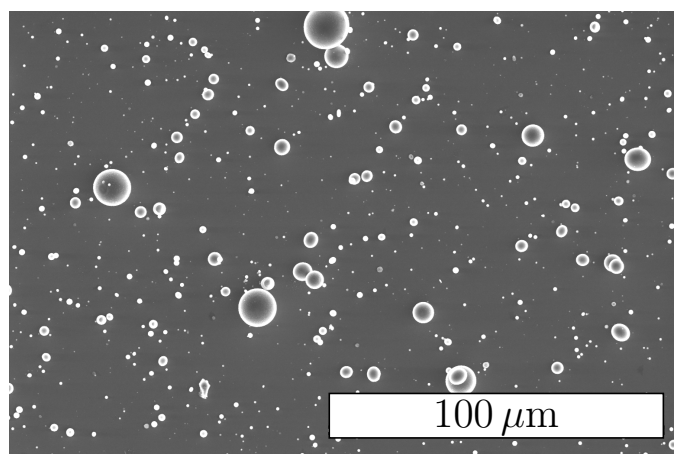

(a) Topview of droplets on a $\mathrm{Mg}$ thin film

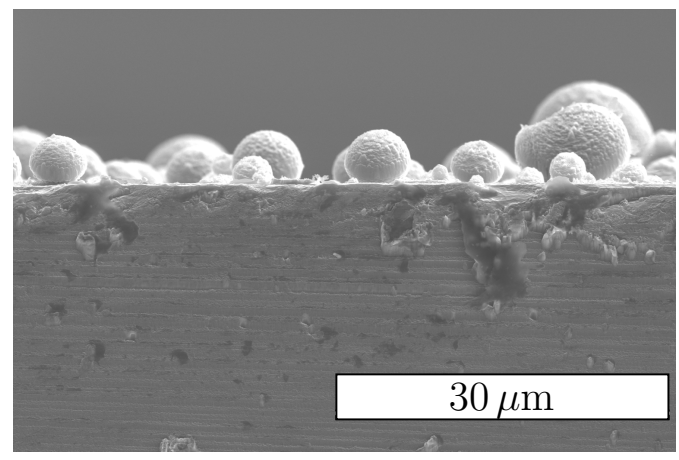

(b) Sideview of droplets on a $\mathrm{Mg}$ thin film

Figure 3.1.: Raster electron microscopy images of thin $\mathrm{Mg}$ films with droplets. The films were produced at laser fluences of $2.08 \mathrm{~J} / \mathrm{cm}^{2}$.

The initial samples, prepared by F. Schlenkrich, were produced at a target-tosubstrate distance of $0.04 \mathrm{~m}$ and laser fluences between $1.14 \mathrm{~J} / \mathrm{cm}^{2}$ and $2.08 \mathrm{~J} / \mathrm{cm}^{2}$. However, these samples showed a strong formation of metal droplets on the surface as can be seen in figure 3.1. This strong droplet formation is a result of the low melting point of magnesium in combination with the high energy densities of the PLD technique [196]. The droplets are a major obstacle for diffusion measurements. A thin film with large droplets can neither be regarded as plate nor as spheres, but a mixture of both, making it difficult to apply a single solution of Fick's law (for example those discussed in chapter 3.2.3). Hence, the deposition process had to be improved to prevent the droplet formation.

As shown in figure 3.2 (a) the droplet density decreases with the average laser fluence. In addition, a view on the target (shown in figure 3.2(b)) clarifies the situation. The laser spot roughly has a Gaussian energy distribution as schematically shown in figure 3.2 (b). Therefore, at the center of the target the higher intensity leads to a more pronounced expansion of the molten zone and an enhanced surface roughness. The melting results in metal extrusions on the target. These lead to droplets on the sample. Overall, a lowering of the energy density of the laser spot leads to an almost droplet free sample. To reach energy densities low enough for a droplet free sample the laser fluence needs to be in the range of $0.4 \mathrm{~J} \mathrm{~cm}^{-2}$ (compare figure $3.2(a))$. Originally the energy density was limited by the laser power and could not be reduced far enough. However, changes to the PLD system done by A. Dittrich et al. allow to defocus the laser beam on the target [197]. This stretches the Gaussian energy distribution and lowers the laser fluence.

Using the thusly improved PLD system, F. Döring prepared new samples. Mg films 


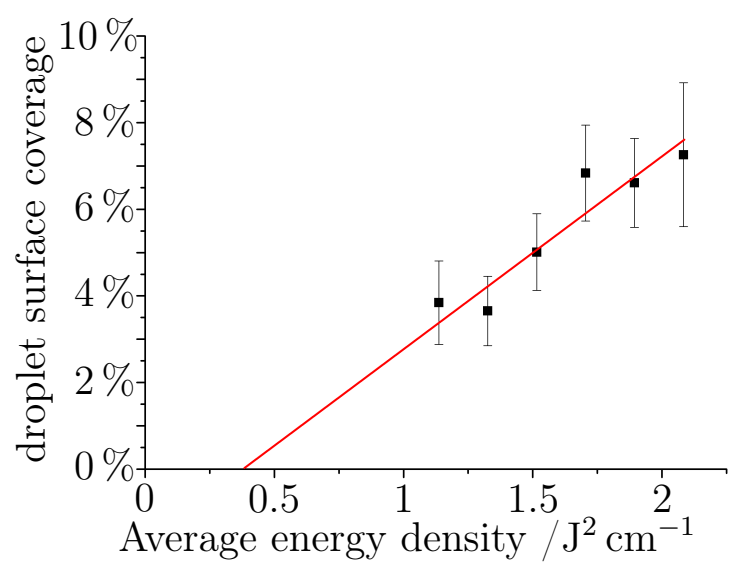

(a) Droplet surface coverage in comparison with laser fluence

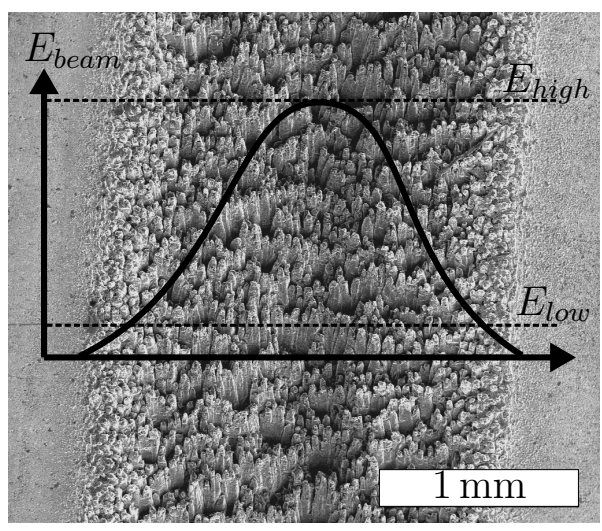

(b) PLD magnesium target after deposition

Figure 3.2.: Influence of laser beam energy on deposition on Mg. Figure (a) shows how a decrease of the average energy density decreases the surface coverage of droplets on $\mathrm{Mg}$ thin films (evaluated by SEM). This can be explained by figure (b) which shows a magnesium target after deposition (SEM image by F. Schlenkrich). The Gaussian energy density of the laser spot is schematically shown. At positions of high energy density $\left(E_{\text {high }}\right)$ the target melts and produces extrusions that result in droplets on the sample. Areas of low energy density $\left(E_{\text {low }}\right)$ produces no droplets.

of about $400 \mathrm{~nm}$ thickness were prepared at laser fluences of $1.7 \mathrm{~J} \mathrm{~cm}^{-2}$. The targetto-substrate distance was again $0.04 \mathrm{~m}$. The resulting films were found to be droplet free. Initially, a Pd layer had to be added after the PLD preparation. For this the $\mathrm{Mg}$ thin films were transfered into the argon-ion beam sputter system presented in section 3.1.1. The film was first placed in the path of the ion beam to remove the surface oxide, which forms during transport [108, 109]. The ion beam removes about $0.8 \mathrm{~nm} / \mathrm{s}$. A green glow is visible in the violet argon-ion beam during the oxide removal, thus giving an indication when no surface oxide is left. Afterwards a Pd capping layer was deposited with the same sputter conditions as given above. The deposition of the $\mathrm{Pd}$ capping layer in a separate system is an unnecessary difficulty, since one has to be careful not to remove the whole $\mathrm{Mg}$ thin film on the one side and not to leave a surface oxide, which prevents hydrogen uptake (compare section 2.1.4). Therefore, the deposition of palladium was also integrated into the PLD system. A Pd target of a purity of $99.9 \%$ was used. S. Hoffmann-Urlaub prepared the final samples measured by gas volumetry measurements by producing thin $\mathrm{Mg}$ films of a thickness of around $100 \mathrm{~nm}$ using the parameters of F. Döring (laser fluence of $1.7 \mathrm{~J} \mathrm{~cm}^{-2}$, target substrate distance of $6.5 \mathrm{~cm}$ ). Afterwards, a Pd film of a thickness of about $30 \mathrm{~nm}$ was added on top with the same deposition parameters. 


\subsection{Gas volumetry}

This chapter introduces the main experimental setup which is utilized to determine diffusion coefficients, the gas volumetry. The principle is simple: the pressure change of a gas atmosphere is measured in a fixed volume. The pressure changes if gas is taken up by a sample or leaves the sample. This allows evaluating of different parameters. On the one side, the gas concentration in the sample can be evaluated after equilibrium is reached. Additionally, the gas volumetry allows evaluating of the diffusion constant by applying an appropriate solution of Fick's second law as will be discussed in section 3.2.2. After introducing the setup itself in section 3.2.1 a typical measurement, including its theoretical description is described. Finally, the algorithm employed to evaluate the recorded pressure data for the diffusion coefficient is summarized.

\subsubsection{Gas volumetry setup}

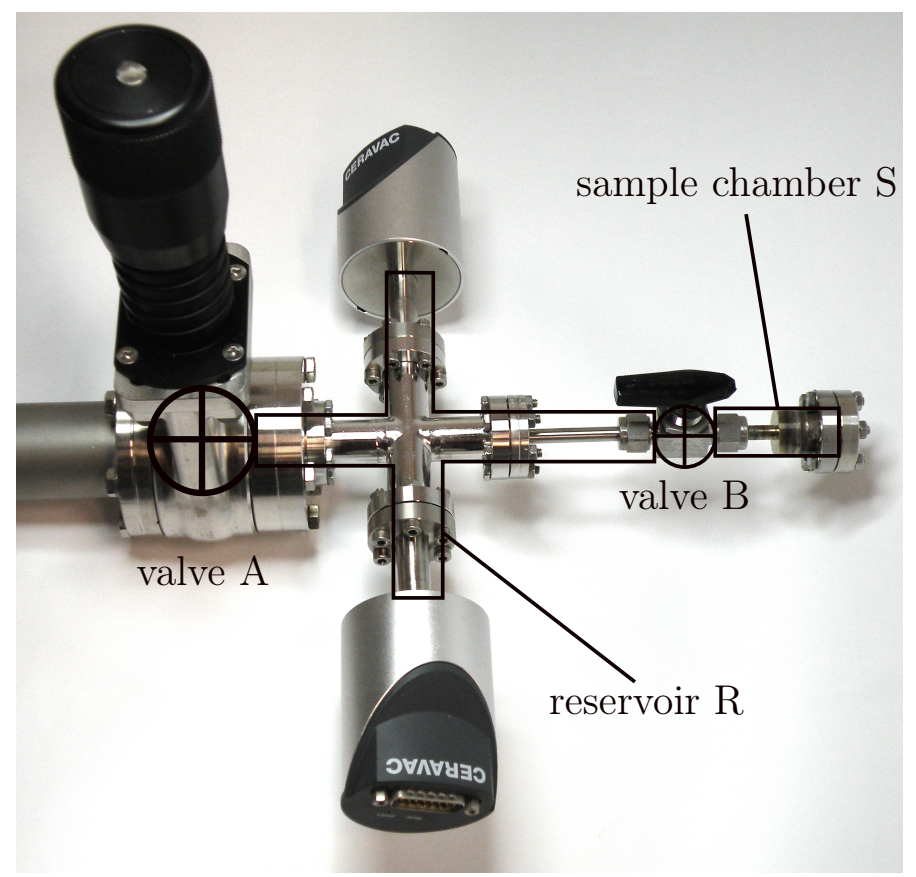

Figure 3.3: Gas volumetry setup utilized in this work. The reservoir $\mathrm{R}$ can be flooded with hydrogen gas or pumped down by opening valve A. Valve B connects the reservoir to the sample chamber S. At the end of the reservoir two CERVAC baratron pressure gauges are connected. One covers the range of 0.01 torr to 1 torr, the other covers the range of 1 torr to 100 torr.

Figure 3.3 shows the gas volumetry setup. It consists of two chambers: the reservoir $\mathrm{R}$ and the sample chamber $\mathrm{S}$, divided by a valve (valve B). They are connected to a high vacuum system by an additional valve $\mathrm{A}$. Opening valve $\mathrm{A}$ allows pumping down both chambers to a background pressure of $p_{\text {back }}<5 \cdot 10^{-5} \mathrm{~Pa}$. Hydrogen gas is introduced by valve $\mathrm{A}$. The hydrogen pressure can be increased, starting from the background pressure, up to $10^{3} \mathrm{hPa}$. The reservoir volume was measured by a defined volume $\mathrm{M}$ that was connected to the setup. The reservoir was thereafter filled 
with a known pressure of hydrogen and its volume increased by the known volume M. The measured pressure drop for different hydrogen pressures allowed to measure the reservoir volume $\mathrm{R}$ as $V_{R}=(83 \pm 2) \mathrm{cm}^{3}$. The same procedure was repeated for the sample chamber $\mathrm{S}$ and its volume was measured as $V_{S}=(5.4 \pm 0.2) \mathrm{cm}^{3}$. In addition, one directly measures the volume ratio of the reservoir $\mathrm{R}$ divided by the combined volume of sample chamber $\mathrm{S}$ and the reservoir R: $\Delta V=\left(V_{R}\right) /\left(V_{R}+V_{S}\right)=$ $0.939 \pm 0.001$. This allows predicting the pressure drop $\Delta p_{0}$, because of the increasing volume, after opening valve B. Assuming an ideal gas it is equal to the ratio $\Delta V$. The pressure measurement for this, as well as for the diffusion measurements in this work utilized two CERVAC baratron pressure gauges, type CSR100. One allows measuring a range from 0.01 torr to 1 torr $(\approx 0.013 \mathrm{hPa}$ to $1.33 \mathrm{hPa})$, while the other measures a range from 1 torr to 100 torr $(\approx 1.33 \mathrm{hPa}$ to $133.32 \mathrm{hPa})$.

\subsubsection{Theoretical description of a gas volumetry measurements}

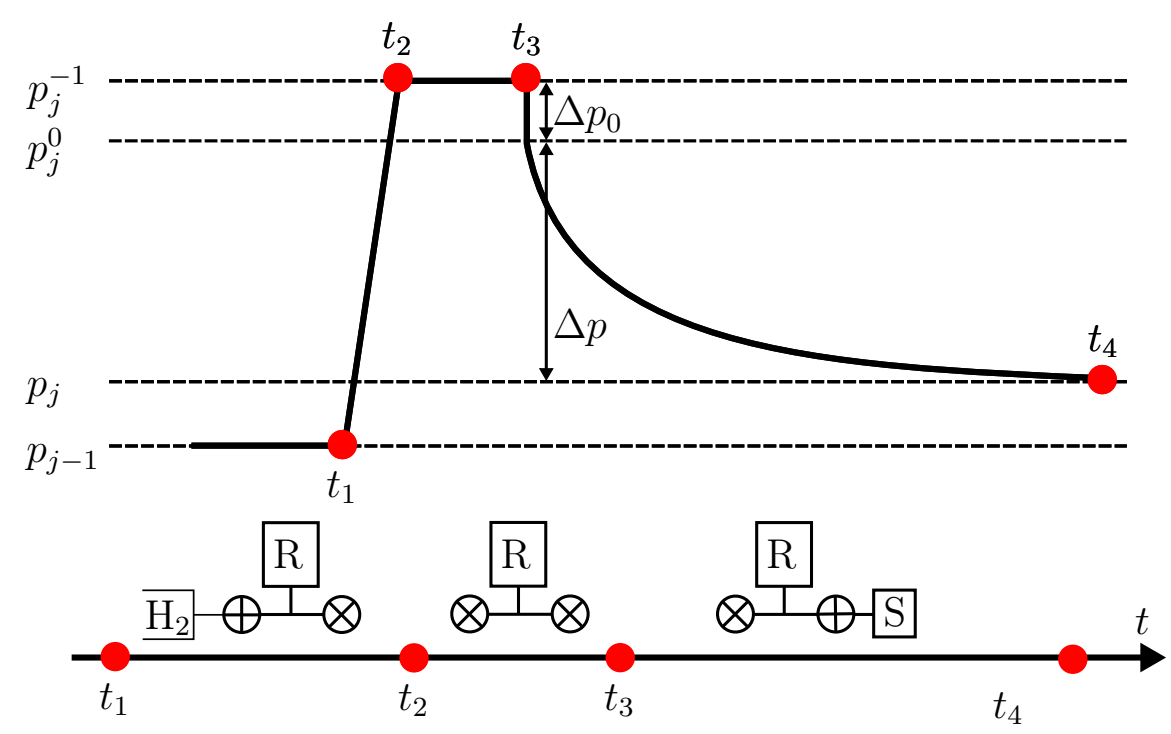

Figure 3.4.: Steps of gasloading procedure and the related pressure change in the reservoir R. Up until time $t_{1}$ the pressure is $p_{j-1}$. At $t_{1}$ the reservoir is opened on one side to introduce additional hydrogen and the pressure increases up to $p_{j}^{-1}$, which is reached at time $t_{2}$. Afterwards, the valve is closed and the reservoir pressure stays constant. At time $t_{3}$ the second valve to the sample chamber $\mathrm{S}$ is opened. This leads first to a pressure drop to $p_{0}$ because of the increased volume. Afterwards the sample takes up hydrogen, thereby lowering the pressure to the new equilibrium pressure $p_{j}$. The new equilibrium is reached at time $t_{4}$, at which point the procedure can be repeated.

Figure 3.4 shows the gas loading procedure applied in this work. In the beginning the reservoir $\mathrm{R}$ (and the sample chamber $\mathrm{S}$ ) are at a pressure $p_{j-1}$. This may be 
the background pressure of $p_{b a c k}<5 \cdot 10^{-5}$ or the pressure reached by any previous loading step. At time $t_{1}$, by opening valve $\mathrm{A}$ (and closed valve $\mathrm{B}$ ) the pressure in the reservoir is increased to a new pressure $p_{j}^{-1}$. Afterwards at time $t_{2}$ valve $\mathrm{A}$ is closed and the reservoir contains a known volume of hydrogen gas. At time $t_{3}$ valve $\mathrm{B}$ is opened and the pressure drops according to $\Delta V=\Delta p_{0}$. At later times the sample in $\mathrm{S}$ may take up additional hydrogen, leading to an additional drop of $\Delta p$. Finally, at time $t_{4}$ the pressure reaches a new equilibrium $p_{j}$. In the following, the procedure may be repeated for additional loading steps.

Hydrogen concentration evaluation The pressure drop $\Delta p$ allows evaluating the amount of hydrogen the sample has taken up in a certain time. The concentration can be calculated using the ideal gas law [198]:

$$
p V=N k_{B} T \text {. }
$$

Where $p$ and $V$ are pressure and volume, $k_{B}$ is the Boltzmann constant, $T$ the temperature and $N$ the number of gas molecules. The number of hydrogen atoms taken up by the sample is equal to two times the number of hydrogen molecules in the chambers at time $t_{3}$ minus the number of hydrogen molecules at the new equilibrium at time $t_{4}$. Combining this with the ideal gas law (equation 3.1) leads to:

$$
N_{H i n M}=2 \frac{V}{k_{B} T} \Delta p
$$

Dividing the number of hydrogen atoms by the number of metal atoms of the sample gives the concentration of hydrogen per metal atom:

$$
c_{H}=\frac{V_{M}^{m}}{V_{M} N_{A}} 2 \frac{V}{k_{B} T} \Delta p .
$$

$V_{M}^{m}$ and $V_{M}$ are the molar volume and volume of the metal sample. $N_{A}$ is the Avogadro constant.

Diffusion from a gas atmosphere: The well-stirred fluid of limited volume The pressure drop $\Delta p$ not only allows evaluating the concentration in the sample at a given time. Because it contains information about the uptake over time it allows evaluating the diffusion coefficient. For this Fick's second law (equation 2.11) has to be solved. In this work thin films are measured, which can be assumed to be infinite slabs with thickness $L$. Hydrogen is assumed to only enter from the palladium covered side. Initially the concentration in the sample is $c_{\text {sample }}=0$ and the outside concentration is set to $c_{g a s}=c_{0}$. In addition, it is assumed that for all times the concentration outside of the sample is not dependent on the position. This condition is known as a well-stirred fluid as it is true for a fluid that is constantly stirred 
[199, 200, 201. However, it is an easily fulfilled assumption for gases, because of the high mobility of the gas molecules. The final boundary condition can be formulated as:

$$
\lambda L \frac{\partial c}{\partial x}=\mp D \frac{\partial c}{\partial x}
$$

with $\lambda=V_{\text {fluid }} / K V_{\text {slab }}$ being the effective volume ratio of the fluid in comparison to the slab. $K$ is the partition coefficient, a measure for the solubility of the reservoir and the slab. This boundary condition states that all change in concentration in the limited reservoir comes from diffusion into or out of the slab.

Diffusion from a limited volume of well-stirred fluids was first solved by Carslaw and Jaeger, originally for the comparable problem of heat transfer [200]. Carman and Haul wrote down several convenient forms to measure diffusion coefficients on basis of the well-stirred fluid conditions [199]. The general solution is given by [199, 200, 201]:

$$
1-\frac{M}{M_{\infty}}=\sum_{m=1}^{\infty} \frac{2 \lambda(1+\lambda)}{1+\lambda+\lambda^{2} q_{m}^{2}} \exp \left(-\frac{q_{m}^{2} D t}{L^{2}}\right)=\sum_{m=1}^{\infty} Z_{m} \exp \left(-\frac{q_{m}^{2} D t}{L^{2}}\right) .
$$

$M$ is the total amount of absorbant absorbed in time $t$ and $q_{m}$ are the successively increasing non-zero roots of the equation $\tan (q)+\lambda q=0$. For gases there is a dependence on the absorbed amount to the pressure $p$ at a given time in the reservoir:

$$
1-\frac{M}{M_{\infty}}=\frac{p-p_{j}}{\Delta p}
$$

$p_{j}$ and $\Delta p$ are the same as discussed above and shown in figure 3.4 . As said before, $\lambda$ is correlated to the effective volume ratio between reservoir and slab. For gases it can be written as:

$$
\lambda=\frac{p_{j}-p_{j-1}}{\Delta p} .
$$

This solution assumes that $D$ and $K$ are constant during the diffusion process. This may not be the case in real experiments. Carman and Haul therefore proposed that during loading from a gas atmosphere a single loading step should be kept small enough that a constant $D$ and $K$ can be assumed [199].

While equation 3.4 is the full solution for diffusion from a limited volume of a wellstirred fluid, it is not easily applicable to measurement data. Carman and Haul showed that the first term already approximated the full solution well if $\lambda>0.4$ [199]. For this it is helpful if the chamber volume is not too much larger than the sample volume. Under these conditions a significant amount of volume from the fluid is taken up, even if $K$ is small, meaning the sample has a relatively small solubility 


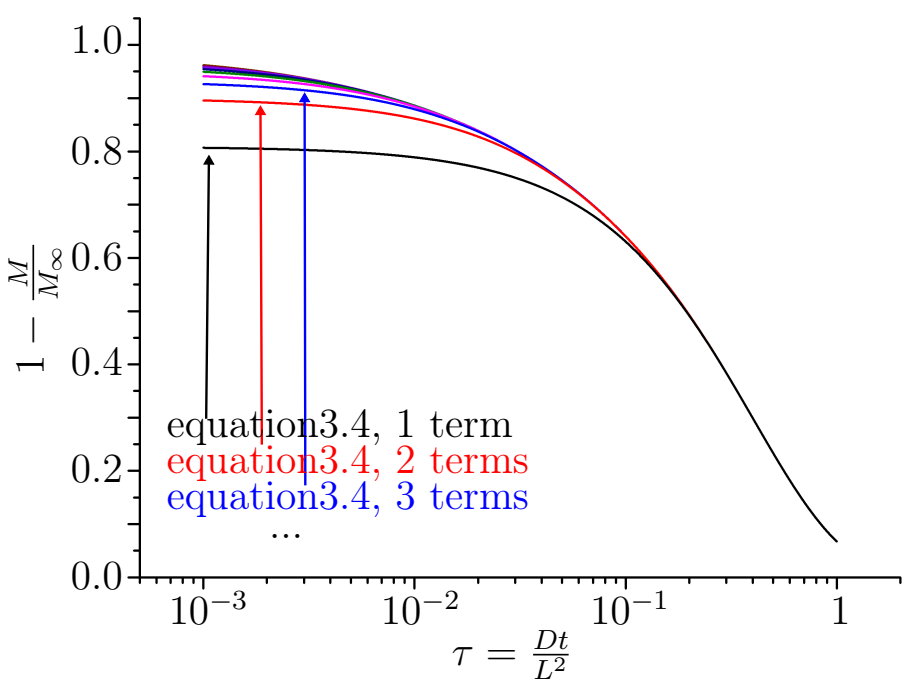

Figure 3.5: $1-M / M_{\infty}$ calculated by equation 3.4 . but by aborting the infinite sum after $n$ terms. The values are plotted as function of the logarithm of $\tau=$ $D t / L^{2}$. The black line contains only the first term. The red line contains two terms. The blue line contains three terms and so on. Overall lines for equation 3.4 up ten terms are plotted. The main contribution of additional terms after the first are for small $\tau$, with decreasing influence of each following term.

for the solvent. Therefore, the combined volume of the reservoir chamber and sample chamber was kept as small as possible (compare chapter 3.2.1). Figure 3.5 shows the infinite sum in equation 3.4 calculated up to different values of $m$. The first term approximation cannot be separated from calculations with more than one term for values of $\tau=D t / L^{2}>0.1$. For smaller values the full solution can be approximated by a short-term approximation calculated by a Laplace transformation [199]:

$$
1-\frac{M}{M_{\infty}}=(1+\lambda) \exp \left(\frac{\sqrt{\frac{D t}{L^{2}}}}{\lambda}\right) \operatorname{erfc}\left(\frac{\sqrt{\frac{D t}{L^{2}}}}{\lambda}\right)-\lambda+\ldots
$$

This approximation can be developed further for small or large values of $\sqrt{\tau} / \lambda$ by a Taylor expansion.

Ma and Evans studied how much real geometrical objects differ from the perfect infinite slab [202]. They found that for a large volume ratio of the sample compared to the chamber the infinite slab solution gives a good approximation to a real rectangular prism. In addition they showed that the shape does not influence the uptake behavior if $D t / \sigma^{2}<0.2$. $\sigma=V_{\text {sample }} / A_{\text {sample }}$ is the ratio of the sample volume to its surface. For thin films on a substrate, $\sigma$ can be approximated as half of the film thickness $d$. Because $d$ is very small, the infinite slab is a very good approximation for thin films, even for times up to $10^{5} \mathrm{~s}^{1}$

Diffusion from a gas atmosphere: other boundary conditions While most experiments were done in the gas volumetry setup as presented above (figure 3.3),

\footnotetext{
${ }^{1}$ For this value a diffusion coefficient of $D \approx 10^{-18} \mathrm{~m}^{2} \mathrm{~s}^{-1}$ has been assumed, taking a rough average of the values for hydrogen diffusion influenced by $\mathrm{MgH}_{2}$ as shown in figure 2.6.
} 
some used different setups. In these cases the boundary conditions assumed in the solution of diffusion from a well- stirred fluid of limited volume may not be fulfilled. Therefore, another solution of Fick's second law applied in this work is shortly introduced in the following.

In some cases the limited volume assumption was not realistic. This can be the case if the chamber volume is much larger than the sample volume. The extreme case would be a constant flow of gas at a given pressure. Under these conditions it is better to assume a constant surface concentration $c_{\text {const }}$ outside the sample instead of a limited volume. The solution of diffusion from a constant surface condition into an infinite slab is given as [201]:

$$
1-\frac{M}{M_{\infty}}=\sum_{m=0}^{\infty} \frac{8}{(2 m+1)^{2} \pi^{2}} \exp \left(-\frac{D(2 m+1)^{2} \pi^{2} t}{4 L^{2}}\right) .
$$

Again, the first term of the infinite sum was taken to be an appropriate approximation of the full solution.

\subsubsection{Developed algorithm to evaluate $D$ from gas volumetry measurements}

In the following section the steps taken to evaluate a single hydrogen gas loading step are collected. A graphical overview is additionally given in figure 3.6. The general change during a gas volumetry step was already introduced above and is shown in figure 3.4 .

Step 1: correction of leakage It is possible that for long times the pressure in the gas volumetry setup increases (see figure 3.6, step 1). The gas volumetry setup is isolated after the pressure is set and some leakage will always occur. For the evaluation it is important that the gas volume stays the same (to achieve the boundary conditions for diffusion from a limited volume), therefore no active pumping or gas flow is possible. If the pressure increases this increase is corrected by fitting an appropriate function to it. This may be a simple straight line $(p=m t+b, b$ being the $y$-axis intersect and $m$ being the slope) or an exponential increase if it stabilizes at large times $\left(p=p_{0}+A \exp (x / \tau)\right.$, with $A$ being the amplitude, $\tau$ being the time constant and $p_{0}$ being the maximum pressure reached). The fitted curve is then subtracted from the pressure resulting in a corrected pressure $p_{\text {corr }}$. This first step may not always be necessary. The setup employed here was able to hold a pressure between $1 \mathrm{hPa}$ and $100 \mathrm{hPa}$ for several days. However, when the resistance stage (see chapter 3.3) was connected a measurable leakage occurred. 
Step 2: normalization of pressure The corrected pressure (if a correction was necessary) is afterwards normalized to values between zero and one (see figure 3.6, step 2). For this an exponential decay $\left(p=p_{0}+A \exp (-x / \tau)\right.$, with $A$ being the amplitude, $\tau$ being the time constant and $p_{0}$ being the minimal pressure reached) is fitted to the pressure drop. In theory the drop $\Delta p_{0}$, coming from the volume increase by combining the reservoir and sample chamber, should be removed beforehand. However it was found that the exponential decay fits well and excludes $\Delta p_{0}$ even if it is not removed manually. The pressure can be recalculated as:

$$
p_{\text {nor }}=\frac{p_{\text {cor }}-p_{0}}{A} \stackrel{!}{=} \frac{p-p_{j}}{\Delta p} .
$$

The last equation states that the normalized pressure $p_{\text {nor }}$ should be equal to $\left(p-p_{j}\right) / \Delta p$ which is equal to $1-$ $M / M_{\infty}$.

Step 3: D evaluation As said above $p_{\text {nor }}=1-M / M_{\infty}$. By using the first term approximation of equation 3.4 the diffusion coefficient $D$ can be calculated by fitting the normalized and halflogarithmic plotted pressure $\ln \left(p_{\text {nor }}\right)$.

$$
\begin{array}{r}
p_{\text {nor }}=Z_{1} \exp \left(-\frac{q_{1}^{2} D t}{L^{2}}\right) \\
\Leftrightarrow \ln \left(p_{\text {nor }}\right)=\ln \left(Z_{1}\right)+\frac{q_{1}^{2} D t}{L^{2}} .
\end{array}
$$

One can calculate $Z_{1}$ from $\lambda$ (see equation 3.4 and equation 3.5 and fit a straight line with the $y$-axis intersect $\ln \left(Z_{1}\right)$. $D$ can be calculated from the slope $m$ as $D=\left(L^{2} \cdot m\right) / q_{1}^{2} . L$ is the length hydrogen diffuses in time $t$. This may be equal to the film thickness $d$, but this is not always true. In this work the diffusion length was estimated by an additional

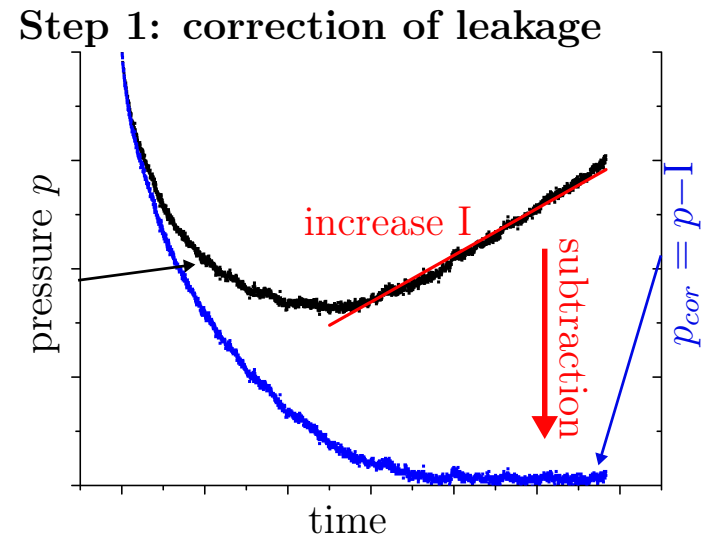

Step 2: normalisation of pressure

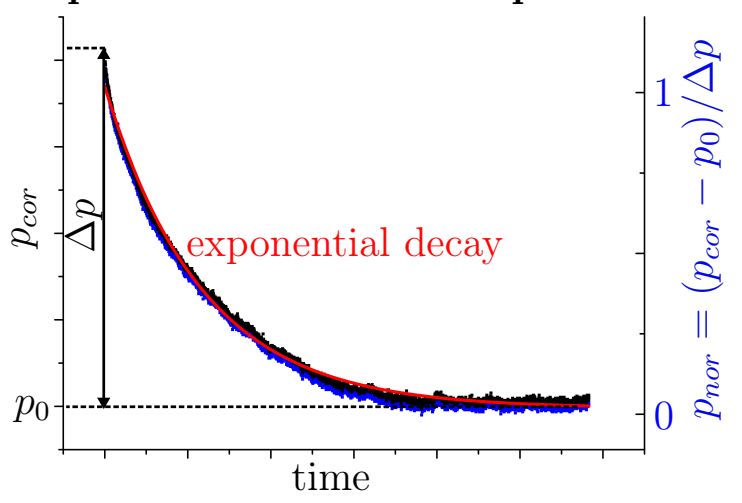

Step 3: D evaluation

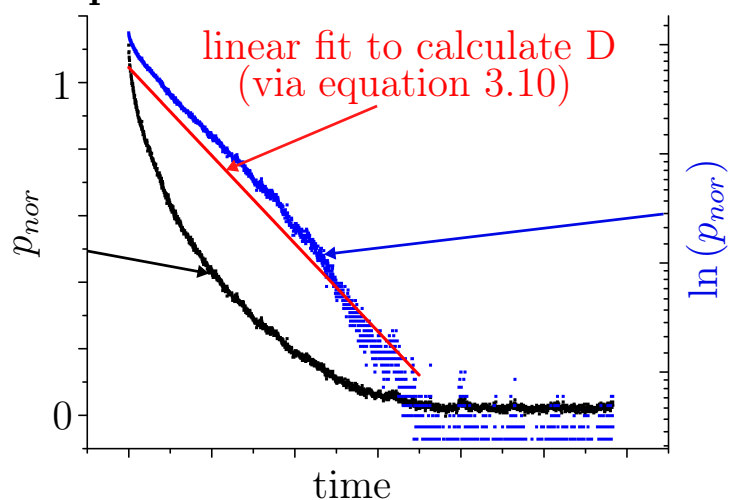

Figure 3.6.: Measures taken to get from the measured data during one pressure step to a diffusion coefficient $D$. Three steps are taken (Step one is not always necessary). For each evaluation step, the value before are shown in black, the values after in blue. 


\section{Applied experimental methods and developed algorithms}

in situ resistance measurement which is presented in section 3.3 . The resistance measurements allows estimating the thickness of the $\mathrm{MgH}_{2}$ layer $d_{\mathrm{MgH} 2}$ at a given time. This thickness is applied as diffusion length $L$ as the hydrogen has to diffuse through the magnesiumhydride layer, which after Uchida et al. forms at the the palladium/magnesium interface and grows into the $\mathrm{Mg}$ film [44]. Therefore, using the thickness of the $\mathrm{MgH}_{2}$ layer as diffusion length should allow evaluating the diffusion coefficient of hydrogen in $\mathrm{MgH}_{2}$. Because of several effects it has to be assumed that the error of the diffusion length $\sigma_{L}$ is large (see below). It was therefore always set to $\sigma_{L}=0.25 \cdot L$. The error of $m$ is taken from the fit. From the change of $q_{1}^{2}$ with $\lambda$ an error of $\sigma_{q_{1}^{2}}=0.2$ was assumed to be realistic (compare figure A.2 in appendix A).

F. Jung conducted a couple of background measurements of the gas volumetry setup containing no sample. His measured data can be found in the appendix $\mathrm{A}$. The two measurements show an exponential decrease of the chamber pressure without sample. The steel of the chamber walls is expected to have a small solubility for hydrogen (see [203] and [204, 205, 206] for the solubility of hydrogen in steel). However, hydrogen may absorb to the chamber walls leading to the measured decrease. Yet, because of the long time scale of the pressure drop this seems unrealistic. More probable is a decrease as the result of a drift of the pressure gauges membranes. Independent of its origin, the pressure drop without sample can be corrected. It is treated similarly to the decay by two decay channels. This is described by a simple exponential decay $p=p_{0} \exp (-\Lambda \cdot t)$, as would be the decay by one channel, with a starting value $p_{0}$ and a decay value $\Lambda$. The difference to one decay channel is that the decay rate for two channels is the sum of the single decay rates $\Lambda=\Lambda_{\text {zero }}+\Lambda_{\text {sample }}$. Taking into account the additional pressure decrease does not change the starting value, but only the overall decay constant. This fact can be applied to the evaluation process, because the slope measured by equation 3.10 should be equal to the decay constant. Hence, the influence of the additional pressure decrease can be corrected by subtracting the average slope of the two zero measurements $m_{\text {zero }}=\bar{m}_{\text {zero }}=7.065 \cdot 10^{-5}$ from the slope measured with sample $m_{\text {cor }}=m-m_{\text {zero }}$. In this work all measurements were corrected in this way. In theory, an additional pressure drop changes also the $y$-axis intersect $\ln \left(Z_{1}\right)$ in equation 3.10 . This is not further treated here, because the additional drop is small compared to the drop by the sample uptake.

\subsection{Resistance measurement}

The following section introduces the four-point resistance measurement utilized to evaluate the volume fraction of the $\mathrm{MgH}_{2}$, the diffusion length of the hydrogen at a given time and diffusion coefficients. The advantage of a four-point resistance measurement, comparing it to a classical two point resistance measurement, is that 
it allows measuring small resistances accurately. At first, the stage employed for the four-point resistance measurements is introduced. In the following the models applied to evaluate the measured resistance for the different parameters are collected. Finally, the algorithm used to evaluate the resistance data is presented.

\subsubsection{Setup}

high vacuum electrical feedthrough

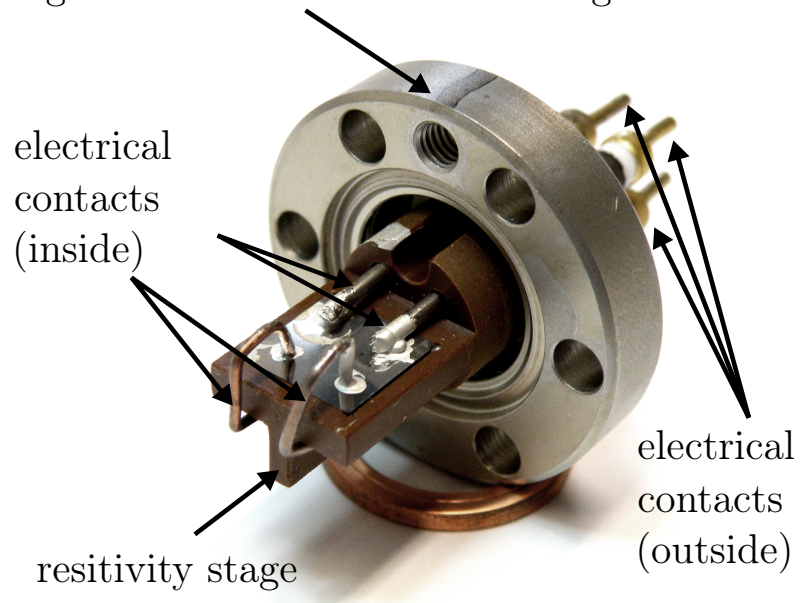

Figure 3.7: Resistance stage utilized for in situ four-point resistance measurements in hydrogen atmosphere. The stage is set onto a high vacuum electrical feedthrough which can be attached to the sample chamber of the gas volumetry setup (see figure 3.3. The sample is positioned into the stage and can be contacted by silver paste to the electrical contacts.

Figure 3.7 shows the resistance stage. The stage is attached to a high vacuum electrical feedthrough that allows connecting the stage to the sample chamber of the gas volumetry setup. The sample is positioned in a cutout of the stage and connected with silver paste. After mounting the stage in the sample chamber it can be contacted from the outside using a Keithley 2000 Multimeter. The resistance is measured in the four-point mode of the Keithley Multimeter and read out automatically using the program "unidaq", written by F. Schulz. The resistance stage does change the sample chamber volume to $V_{S}=(3.5 \pm 1.8) \mathrm{cm}^{3}$ and therefore also the volume ratio $\Delta V=\Delta p_{0}=0.959 \pm 0.0001$.

\subsubsection{Theoretical models developed and applied to evaluate resistance data}

The measured resistance data correlate with the volume fraction of the formed magnesiumdihydride. As discussed in section, 2.1.3 $\mathrm{MgH}_{2}$ is (in bulk) an insulator with a much higher resistance. The specific resistance $\rho_{M g H 2} \approx 10^{2} \mu \Omega \mathrm{m}$ of hydrided $\mathrm{MgH}_{2}$ thin films [104 was found to be much lower than that of grown $\mathrm{MgH}_{2}$ thin films $\left(\rho>10^{-10} \mu \Omega \mathrm{m}[101]\right)$. Yet, the specific resistance of hydrided thin films is 


\section{Applied experimental methods and developed algorithms}

still higher than the specific resistance $\rho_{M g}=65 \mu \Omega \mathrm{m}$ of $\mathrm{Mg}$ thin films [104]. Therefore the increasing resistance found in magnesium films during hydrogen loading is assumed to originate from the forming hydride. Using the nucleation and growth model introduced by H. Uchida [44] it can be assumed that after the initial nucleation a closed blocking layer forms which grows further into the film. This means three layers exist above the silicon substrate: magnesium of decreasing thickness $d_{M g}$, magnesiumdihydride of increasing thickness $d_{M g H 2}$ and palladium of a fixed thickness $d_{M g}$. The overall thickness is $d=d_{P d}+d_{M g H 2}+d_{M g}$. It is assumed that these three layers are contacted in parallel by the four-point measurement. Even if the actual connection is only on the Pd layer this can be assumed, because the distance between the four contacts of the setup is much larger than the distance through the layers. This means that the resistance between the contacts should be much larger that the resistance connecting the three layers.

The overall resistance $R$ as a combination of resistances of the three layers in parallel contact is:

$$
\frac{1}{R}=\frac{1}{R_{P d}}+\frac{1}{R_{M g}}+\frac{1}{R_{M g H 2}}
$$

The resistance can be expressed as $R=\rho \frac{l}{A}$ using the specific resistance $\rho$, the distance between the contacts $l$ and the area $A$ through which the current travels. The specific resistance of the palladium is equal to $\rho_{P d} \approx 150 \Omega \mathrm{nm}$ [207]. For the simple geometry applied here the area can be simplified as the film thickness $d$ and the distance between the contacts should be almost equal for all resistors in equation 3.11. This allows the following formulation of equation 3.11

$$
\frac{d}{\rho}=\frac{d_{P d}}{\rho_{P d}}+\frac{d_{M g}}{\rho_{M g}}+\frac{d_{M g H 2}}{\rho_{M g H 2}} .
$$

Before loading the equation simplifies to:

$$
\frac{d_{0}}{\rho_{0}}=\frac{d_{P d}}{\rho_{P d}}+\frac{d_{M g}}{\rho_{M g}}=\frac{d_{P d}}{\rho_{P d}}+\frac{d_{0}-d_{P d}}{\rho_{M g}} .
$$

Combining equation 3.12 and 3.13 leads to:

$$
\begin{aligned}
\frac{R}{R_{0}} & =\frac{d_{0}}{d} \frac{\rho}{\rho_{0}}=\frac{d_{0}-d_{P d}+d_{P d} \frac{\rho_{M g}}{\rho_{P d}}}{d-d_{P d}-d_{M g H 2}+d_{M g H 2} \frac{\rho_{M g}}{\rho_{M g} 2}+d_{P d} \frac{\rho_{M g}}{\rho_{P d}}} \\
\Leftrightarrow \frac{R_{0}}{R} & =\frac{d-d_{P d}+d_{P d} \frac{\rho_{M g}}{\rho_{P d}}}{d_{0}-d_{P d}+d_{P d} \frac{\rho_{M g}}{\rho_{P d}}}+\frac{d_{M g H 2}}{d_{0}-d_{P d}+d_{P d} \frac{\rho_{M g}}{\rho_{P d}}}\left(\frac{\rho_{M g}}{\rho_{M g H 2}}-1\right) \\
& =A+\frac{d_{M g H 2}}{d_{V F}}\left(\frac{\rho_{M g}}{\rho_{M g H 2}}-1\right) .
\end{aligned}
$$

As discussed in chapter 2.1.2 the $\mathrm{MgH}_{2}$ expands in out-of-plane film direction as its unit cell is larger than the unit cell of the Mg. Therefore, $d \geq d_{0}$ is true for all times. 
$A$ is one at time $t=0$ and afterwards increases. The volume fraction $\phi_{M g H 2}$ of the magnesiumdihydride at a given time is defined in the following, as:

$$
\phi_{M g H 2}=\frac{d_{M g H 2}}{d_{V F}}=\frac{A-\frac{R_{0}}{R}}{1-\frac{\rho_{M g}}{\rho_{M g H}}} \geq \frac{1-\frac{R_{0}}{R}}{1-\frac{\rho_{M g}}{\rho_{M g H}}} .
$$

The final inequality is true because of the expansion of $d$ with time as discussed above. As $A$ is not known at a given time the volume fraction $\phi_{M g H 2}$ is calculated with $A=1$, which underestimates the true volume fraction.

A more realistic volume fraction of the $\mathrm{MgH}_{2}$ would be $\phi_{M g H 2}^{r e a l}=d_{M g H 2} /\left(d-d_{P d}\right)$. However, this can not be calculated from the measurements in this work as $d$ is not known at each point of time. Only $d_{0}$ (the initial thickness) and $d_{\infty}$ (the thickness after loading) are known. However, because $d \geq d_{0}, \phi_{M g H 2}$ will become smaller than $\phi_{M g H 2}^{r e a l}$ at some point in time ${ }^{2}$. It should be noted that this is only true because $\rho_{M g}$ is similar to $\rho_{P d}$. As discussed above the volume fraction $\phi_{M g H 2}$ is underestimated in this work because $A=1$ is assumed for calculations. Using $\phi_{M g H 2}$ instead of $\phi_{M g H 2}^{r e a l}$ only enhances this difference. This is important to keep in mind, but does not hinder the data evaluation. First of all, the volume fraction is studied to evaluate the limiting kinetic process during hydrogen loading of the $\mathrm{Mg}$ films (see also section 2.3). Here, the shape of the curve is important, which is distorted by the increasing underestimation of the true volume fraction. However, the shape of curves that follow different limiting processes differ strongly, so that they can be separated under non-ideal conditions. It may be difficult to extract true kinetic parameters as e.g. the diffusion coefficient, but this is not the goal in this step. The second important use of the volume fraction is the diffusion length, which is calculated from it by multiplying the volume fraction with $d_{V F}$ (see equation 3.14). This means that the diffusion length is also underestimated leading to an underestimation of the resulting diffusion coefficient. This is included in the error of the diffusion coefficients by using an error of the diffusion length of $25 \%$. This error includes the general underestimation, as well as the fact that the model assumes a perfect straight layer. In reality, the layer thickness probably has some dependency on the position in the film. In general, all diffusion coefficients in this work may be slightly higher than the calculated values.

Giebels et al. introduced a model to calculate the time dependent electrical properties [104] in a mixture of two materials. It bases on the Bruggeman effective medium approximation and was also applied by $\mathrm{Qu}$ et al. [140]. The basic equation is given by $\mathrm{Qu}$ et al:

$$
\left(1-\phi_{M g H 2}\right) \frac{\rho_{M g}-\rho}{U \rho+(1-U) \rho_{M g}}+\phi_{M g H 2} \frac{\rho_{M g H 2}-\rho}{U \rho+(1-U) \rho_{M g H 2}}=0 .
$$

\footnotetext{
${ }^{2}$ Because $\left(d-d_{P d}\right)$ will become larger than $d_{V F}$.
} 
Figure 3.8: Magnesiumdihydride volume fraction $\phi_{M g H 2}$ during formation of magnesiumhydride in a magnesium thin film. The black solid line is calculated using the three-layer model, the blue dotted line is calculated using the model by Giebels et al. 104 (see text for further information).

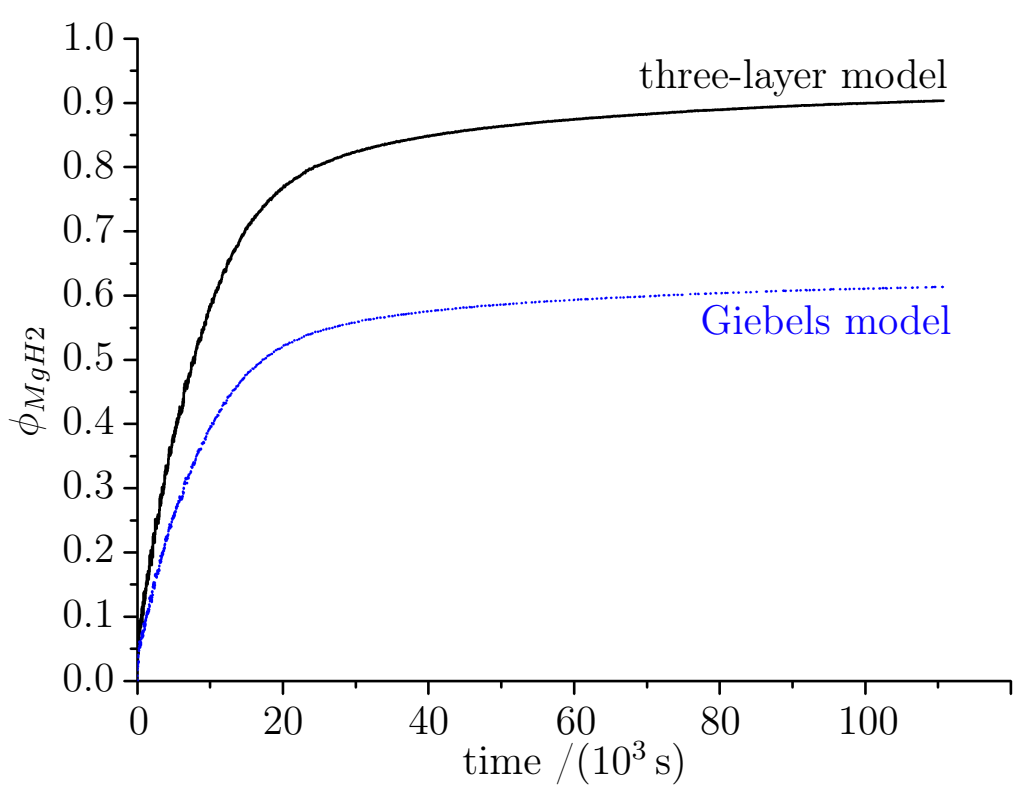

$U$ is a geometrical factor and equals $1 / 3$ for nucleation of spherical hydrides. This can be transformed to:

$$
\phi_{M g H 2} \frac{\rho_{M g H 2}-\rho_{M g}}{\rho_{M g H 2}}=U \frac{\rho}{\rho_{M g H 2}}-U \frac{\rho_{M g H 2}+\rho_{M g}}{\rho_{M g H 2}}-(1-U) \frac{\rho_{M g}}{\rho} .
$$

Because $\rho_{M g H 2}>>\rho_{M g}$ the equation further simplifies to:

$$
\phi_{M g H 2}=U \frac{\rho}{\rho_{M g H 2}}+(1-U) \frac{\rho_{M g}}{\rho}+(1-U) .
$$

$\rho \approx R / d$ can be estimated from the measured resistance. $d$ is the thickness of the combined $\mathrm{Mg}$ and $\mathrm{MgH}_{2}$ layer. This assumes that the length $l$ between the contacts is similar to width $b$. The width forms, together with the thickness $d$, the area $A=b \cdot d$ through which the current has to travel. This model does not include the $\mathrm{Pd}$ capping layer. However, the measured resistance may be corrected for this by using equation 3.11 and $R_{P d} \approx \rho_{P d} / d_{P d}$. The resistance contribution of the palladium layer can be subtracted and afterwards $\rho$ of the combined $\mathrm{Mg}$ and $\mathrm{MgH}_{2}$ layer can be calculated.

Figure 3.8 shows a comparison of the volume fraction $\phi_{M g H 2}$ calculated by the three-layer model introduced above and by the model of Giebels et al. (including the correction for the $\mathrm{Pd}$ capping layer). Because of the approximations done above, the model of Giebels et al. gives a smaller volume fraction $\phi_{M g H 2}$ than the threelayer model for all times. This is an important result, because, as discussed above, the three-layer model already underestimates the true volume fraction. Therefore, the three-layer model seems to be a better choice to calculate the volume fraction of $\mathrm{MgH}_{2}$ in this work. In the following all volume fractions $\phi_{M g H 2}$ are therefore calculated by the three-layer model outlined above. 


\subsubsection{Developed evaluation algorithm for resistance data}

This section collects the different evaluation steps and calculations done after the resistance of the samples is measured over time. All steps and their sequence are shown in the flow chart in figure 3.9. In the following the single steps are explained in more detail.

Measure resistance $R$ over time First the resistance is measured over time. This is done by means of the in situ four-point resistance stage presented in section 3.3.1.

Calculate $\phi_{M g H 2}$ As discussed in section 3.3.2 there are at least two models that allow to calculate the volume fraction of the $\mathrm{MgH}_{2}$ at a given time. In this work only the three-layer model is applied as it seems to be more reasonable. Therefore, the volume fraction is calculated by equation 3.14 .

Evaluate limiting kinetic process The theoretical background for this was introduced in section 2.3. Because the resistance allows calculating the volume fraction at a given time it can be tried to evaluate the limiting kinetic process of the phase transformation from $\mathrm{Mg}$ to $\mathrm{MgH}_{2}$. The algorithm employed here is similar to the one proposed by Pang et al. [185].

As a first step the volume fraction $\phi_{M g H 2}$ is plotted over time $t$ and equation 2.30 is fitted for $\eta$ and $n$. This shows the influence of the impingement on the nucleation and growth process. As discussed in section 2.3 the simple JMAK equation is valid if $\eta \rightarrow \infty$. Therefore, the fit allows predicting if the JMAK equation is a good description of the kinetic process.

If $\eta \rightarrow \infty$ one can plot $\ln \left(\ln \left(1 /\left(1-\phi_{M g H 2}\right)\right)\right)$ over $\ln (t)$. As can be seen by transforming equation 2.29 , in this plot the slope of a straight line fitted to the data gives $n$. $n$ allows evaluating the limiting process, however it should be kept in mind that different processes lead to the same value of $n$ as discussed in section 2.3. In addition, as shown in figure 2.12 (a) values of $n=0.6$ or $n=1.1$ can originate from Jander's model or the contracting volume model respectively. Therefore, these models should be evaluated separately in these cases. Even if $n \neq 0.6$ and $n \neq 1.1$ an evaluation regarding Jander's model or the CV model may offer more insight into the limiting process.

The evaluation of the contracting volume model and Jander's model is in similar fashion. The volume fraction is plotted as function of time $t$ for the CV model and 


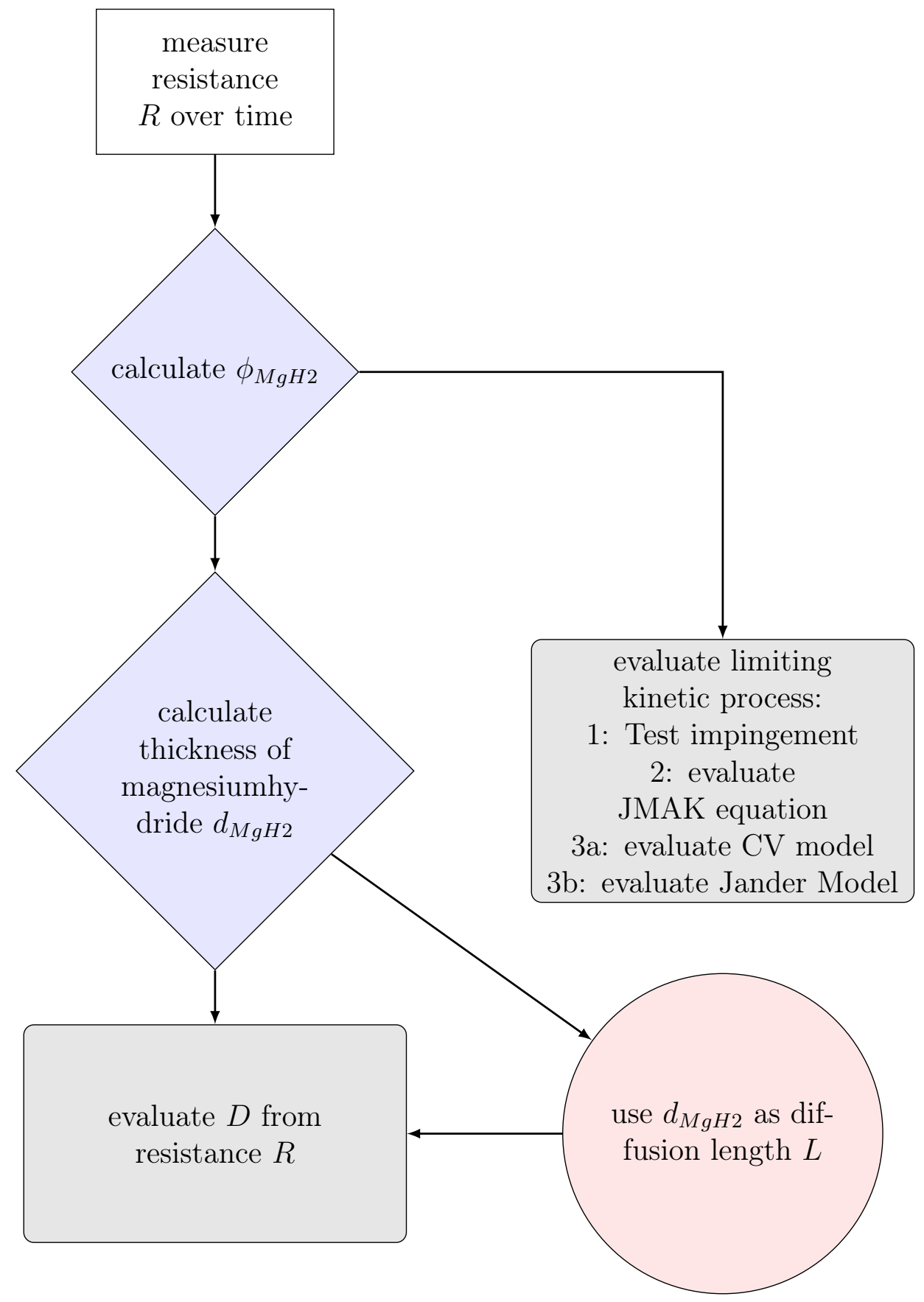

Figure 3.9.: Flowchart of the different steps taken to evaluate the measured resistance $R$. It allows calculating the magnesiumdihydride volume fraction $\phi_{\mathrm{MgH} 2}$ and film thickness $d_{M g H 2}$. The film thickness is also applied as diffusion length to evaluate the diffusion coefficient $D$ from the gas volumetry measurement (see section 3.2). Furthermore, one can calculate the diffusion coefficient $D$ of hydrogen in the magnesiumdihydride and determine the limiting kinetic process of the phase transformation by the resistance data. Additional information for each step is given in section 3.3 .3 . 
of $\sqrt{t}$ for Jander's model. Afterwards, the function $y=1-\left(1-b \cdot\left(x+x_{0}\right)\right)^{d}$ is fitted to both plots. $x_{0}$ and $b$ allow for adjustments if the starting time is incorrect and the maximum volume fraction was miscalculated. $d$ is the dimension of the process. An upper limit for $d$ of 3.5 is set. If the fit converges against the upper limit it is probable that the model does not fit the data. Otherwise the fit quality allows evaluating which model better fits the data. The fit value of $d$ allows evaluating the dimension of the process. If $d>1$ for the Jander model, it should be considered to use other diffusion models, because Jander's model assumes a constant surface area of the diffusion front which is not valid for higher dimensions.

Calculate thickness of $\mathbf{M g H}_{\mathbf{2}} d_{M g H 2}$ Using equation 3.14 allows calculating the thickness of the $\mathrm{MgH}_{2}$ layer $d_{M g H 2}$ at a time $t$ :

$$
d_{M g H 2}=\phi_{M g H 2} \cdot d_{V F} .
$$

$d_{V F}=d_{0}-d_{P d}+d_{P d}\left(\rho_{M g} / \rho_{P d}\right)$ can be calculated using the thickness of the combined thin films $d_{0}$ before loading, the thickness of the palladium layer $d_{P d}$ and the specific resistance $\rho$ of magnesium and palladium.

Use $d_{M g H 2}$ as diffusion length $L$ This is relevant for the next step discussed below (evaluate $D$ from resistance $R$ ) as well as for the evaluation of $D$ from the gas volumetry measurements discussed in section 3.2.3. Using $d_{M g H 2}$ as diffusion length fits to the three-layer model assumed here. For a closed $\mathrm{MgH}_{2}$ layer growing from the top into the $\mathrm{Mg}$ thin film the hydrogen has to diffuse through the $\mathrm{MgH}_{2}$. Therefore the relevant diffusion length is the $\mathrm{MgH}_{2}$ thickness. It should be kept in mind that this is not true before a closed layer is formed. In this case the hydrogen probably diffuses through the magnesium as the diffusivity of hydrogen in $\mathrm{Mg}$ is much higher than the diffusivity in $\mathrm{MgH}_{2}$ (compare figure 2.6).

Evaluate $D$ from resistance $R$ The resistance measurement allows an independent evaluation of the diffusion coefficient $D$. Therefore, $D$ can be evaluated by two different methods (gas volumetry and resistance measurement) at the same time for one sample. The only connection between the two methods is the diffusion length which is calculated from the resistance data and is an input for both methods.

To evaluate $D$ the same models are used that were applied for the evaluation of the gas volumetry measurement, especially the diffusion from a limited volume of well-stirred fluid. Using the calculated volume fraction one gets:

$$
1-\phi_{M g H 2}=1-M / M_{\infty}=Z_{1} \exp \left(-\frac{q_{1}^{2} D t}{L^{2}}\right) .
$$




\section{Applied experimental methods and developed algorithms}

Here, the first-term approximation is applied as in equation 3.9. $D$ is calculated accordingly. This calculation assumes that all the hydrogen taken up by the sample is transformed to hydride. This is a good assumption for the magnesium-hydrogen system because the $\alpha$-phase has a very low solubility of hydrogen (see chapter 2.1.1) and because the phase transformation starts at low concentrations.

\subsection{Electron microscopy}

Electron microscopy is a useful method to study the surface, the grain size and the structure of samples down to the atomic scale. The electron microscopy includes the scanning electron microscope (SEM) and the transmission electron microscope (TEM). For both methods an electron beam is focused on the sample. Further information can e.g. be found in the textbooks by Goldstein et al. [208] and Williams and Carter [209].

For the scanning electron microscopy the beam is scanned over the sample [208]. The electrons interact with the sample by inelastic and elastic scattering. The interaction leads to electrons (and x-rays) that are rescattered (primary electrons) or emitted from the surface (secondary electrons). These can be detected for topographical information and more. SEM studies were conducted to study the surface of the thin films. In addition, an energy dispersive x-ray (EDX) spectrometer allowed to evaluate the chemical composition by detecting the x-rays produced from the interactions of the electron beam with the thin films.

In the transmission electron microscope the electron beam passes through the sample and the electrons are detected below the sample. This allows studies of the crystal structure of samples, using the diffraction of the electrons on the crystal lattices. Further, it allows studies of the chemical composition of samples by measuring their energy loss or the emitted x-rays [209]. TEM studies were the main methods to measure the grain size and grain structure of the different samples before and after hydrogen loading. In addition, one sample was loaded in situ during a TEM study, which allowed to study the hydride formation in magnesium on the micrometer scale. The experimental procedure and the results of the in situ TEM measurement are published in M.Hamm et al. [210].

SEM studies SEM was mainly employed to study the droplet density on the PLD samples as shown in figure 3.1. These studies utilized a secondary electrons detector, if necessary in combination with immersion lens mode for improved resolution. To verify the steel additive concentration of the samples (compare section 3.1.1) EDX spectroscopy was used. For further information see "Scanning Electron Microscopy 
and X-ray Microanalysis" by Goldstein et al. 208]. All these measurements were carried out using a FEI Nova Nano SEM 650. It is equipped with a secondary electrons detector and a backscattered electrons detector. In addition, an Oxford INCA 80mm Energy Dispersive X-Ray spectrometer is attached.

To prepare TEM samples a focused ion beam (FIB) is utilized to cut out cross sections from the thin films. One example is shown in figure 3.10. First a protective platinum layer is deposited with the electron and the ion beam respectively. Afterwards, a cross section is cut out. Using an omniprobe this cross section is transported on a TEM copper grid and attached with platinum. Finally the cross section is thinned. For the preparation of the TEM samples a FEI Nova NanoLab 600 Dual Beam was utilized. It combines a $\mathrm{Ga}^{+}$FIB with a high resolution scanning electron microscope. In addition it contains a platinum and TEOS deposition source and an omniprobe for micromanipulation. The FIB preparation was supported by T. Schulz, who prepared several cross sections and by V. Radisch who added much helpful input for an optimal preparation, especially for the in situ experiment.

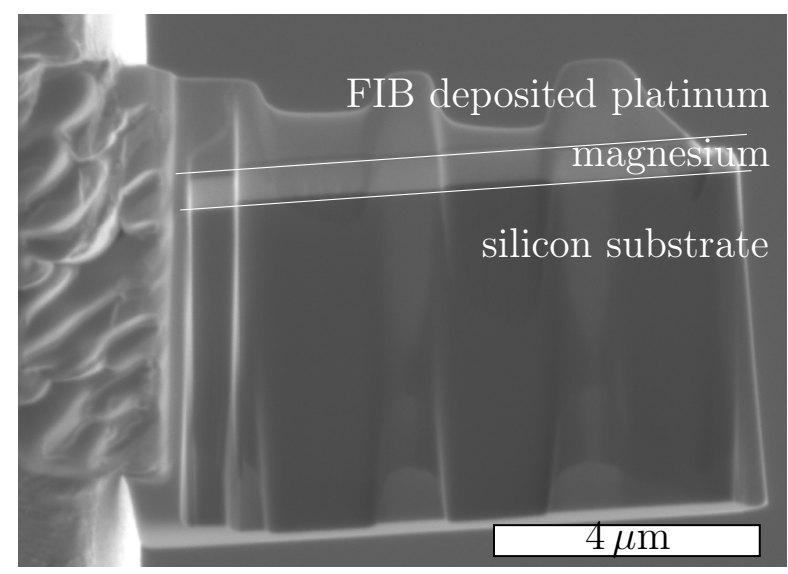

Figure 3.10: Example of a TEM cross section, prepared by FIB. Shown is the magnesium thin film on the silicon substrate. The palladium layer (thin dark grey line) is hardly visible, because of its small thickness. On top platinum was deposited to protect the thin film during the cutting and thinning procedure of the cross section. The cross section shown here is of sample IBSETEM (see table 4.1). The figure is reproduced from M.Hamm et al. [210].

TEM studies The TEM studies were the main method to study the grain size and film thickness before and after hydrogen loading. Additional information about the TEM and its possibilities can be found in the book "Transmission Electron Microscopy" by D.B. Williams and C.B. Carter [209]. During the TEM studies images of different resolutions were taken. Especially helpful were dark field (DF) images which allowed to differentiate single grains. DF imaging utilizes the electrons diffracted at the sample. Grains in the sample, that exhibit a different orientation to the primary beam, can therefore be visible in specific diffraction spots. Selecting one diffraction spot allows identifying individual grains from their surrounding neighbors. The grain size of these isolated grains was measured with the open source program FIJI [211]. Other modes used in this work were bright field (BF) microscopy. for which the unscattered electron beam is imaged and full field (FF) microscopy, which uses all electrons and is typically combined with HR measurements. In addition, diffraction images were taken. For the grain structure and grain 
size studies two TEMs were utilized. The first was a FEI/Philips CM-12T, which is operated at $120 \mathrm{kV}$. It is equipped with a twin lens and a MegaView CCD camera. The second is a FEI/Philips CM-120, also operated at $120 \mathrm{kV}$ and equipped with a twin lens and a Gatan Bioscan CCD camera. The TEM studies were supported by M. Deutges, M.Tiegel, M. Bongers and P. Klose, who operated the TEMs during the grain structure and the grain size studies.

\subsubsection{TEM: in situ hydrogen loading}

For in situ measurements an environmental TEM (Titan E-TEM 80-300) was utilized. The microscope has an image Cs corrector, a Fei X-FEG module, a monochromator and a post-column Quantum 965ER Gatan Image Filter. It was operated at $300 \mathrm{kV}$. The in situ loading was done in coll. with M. Bongers, V. Roddatis and M. Hahn. It allows injecting hydrogen gas into the microscope column during operation of the TEM. For the in situ loading experiment the gas flow was set to maximum, resulting in a hydrogen pressure of about $6.5 \mathrm{hPa}$. After setting the pressure the beam was blanked using a shutter below the electron source (column valves). Images were taken from time to time, which allowed to investigate the development of the hydride over time with minimal electron beam influence. This procedure was chosen as the literature states that $\mathrm{MgH}_{2}$ can be destabilized under the electron beam leading to hydride decomposition [212. Dark field and bright field images were taken at every time step. For this the diffraction spots of the original $\mathrm{Mg}$ diffraction pattern were used, their position having been saved before the hydrogen loading began.
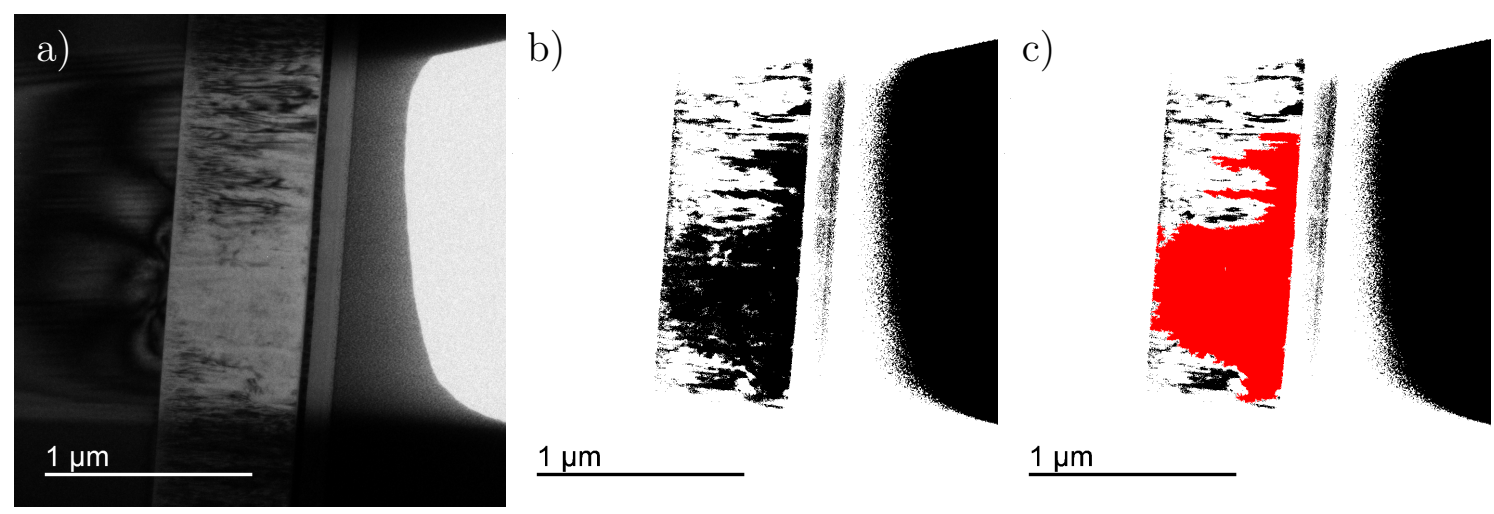

Figure 3.11.: Example of the hydride area $A$ evaluation. a) The original ETEM image, with hydrided area visible as bright area. b) The image is thereafter transformed with the MaxEntropy algorithm in FIJI. c) the "analyze particle" measurement routine of Fiji measures the hydride area $A$ (marked in red). The figure is reproduced from M.Hamm et al. [210].

The images have been evaluated afterwards with FIJI [211]. The process is exem- 
plarily shown in figure 3.11. The original image is shown in figure 3.11 a). At first, the images were converted to black and white, using the MaxEntropy algorithm $3^{3}$ 213. Finally, the "analyze particle" command of FIJI was applied, which identifies particles (here, hydrided areas after binarization) and measures their area $A$. The minimum area counted was 10000 pixels as all smaller particles were found to be artifacts originating from the binarization. This procedure gave the area $A$ of the hydride as a function of time. The area was normalized afterwards to get the volume fraction of the hydride $\phi_{M g H 2}$ over time:

$$
\phi_{M g H 2}=\left(A-A_{0}\right) /\left(A_{\infty}-A_{0}\right) .
$$

This assumes that the hydrided area $A_{0}$ before loading is zero. In addition, it is also assumed that the measured area is completely hydrided at the end of the measurement at time $t=t_{\infty}$. However, binarization may result in wrong starting values larger than zero. As an alternative, a fixed area $A_{\text {eval }}$ can be defined, containing only magnesium. Thermal shift may move this area in the images, which can be adjusted manually in FIJI. The hydrided area $A$ can be normalized by the evaluated area $A_{\text {eval }}$. The resulting volume fraction over time can be evaluated for the limiting kinetic process (see section 2.3 and 3.3.3). For the above discussed procedure it is assumed that the magnesium is fully hydrided in beam direction. Therefore, the two-dimensional view one has in the TEM gives the volume fraction and not only the surface area fraction. This may not be fulfilled in reality and is not assumed for the EELS evaluation. However, the error originating from assuming that the hydride surface is equal to the hydride volume seems to be less impactful than the error originating from the binarization. The reason may be that the TEM images used here have less resolution than the STEM images used for the EELS evaluation.

Additionally to the TEM studies, energy electron-loss spectroscopy (EELS) measurements were carried out during the in situ loading. For this, the energy loss $\Delta E$ of the primary electrons is measured after passing through the sample. The main reason for the energy loss are inelastic scattering events. Plotting the intensity of electrons for a given energy loss gives a large Zero-loss peak, originating from electrons passing through the sample without losing energy. Behind the Zero-loss peak a characteristic spectrum follows, which contains information on the chemistry of the sample. Additional information about EELS can be found in the book "Electron Energy-Loss Spectroscopy in the Electron Microscope" by R. Egerton [214]. EELS was used to spatially map the low-loss region, approximately between $-10 \mathrm{eV}$ and $90 \mathrm{eV}$, in the $\mathrm{Mg}$ film. A spectrum imaging (SI) pattern was selected in regions of interest and, here, the low-loss spectra were acquired.

The plasmon parts, approximately between $6 \mathrm{eV}$ and $30 \mathrm{eV}$, in the individual EEL spectra are specific for $\mathrm{Mg}$ and the $\mathrm{MgH}_{2}$. Paik et al. reported on a bulk plasmon

\footnotetext{
${ }^{3}$ Other algorithms of FIJI were tested, but the MaxEntropy algorithm was found to be the best at distinguishing hydrided areas from magnesium.
} 


\section{Applied experimental methods and developed algorithms}

peak of the $\mathrm{Mg}$ at $10.5 \mathrm{eV}$ and a bulk plasmon peak of the $\mathrm{MgH}_{2}$ at $14.6 \mathrm{eV}$ [212. Therefore, the analysis of the plasmon region allows distinguishing between the spatial region of the $\mathrm{Mg}$ film that has not and the region that has transformed to a hydride. In the SI pattern, each EEL spectrum can either be described by the individual plasmon contributions of $\mathrm{Mg}$ and $\mathrm{MgH}_{2}$ or a superposition of both. This finally allows estimating the volume fraction $\phi_{M g}$ and $\phi_{M g H 2}$ of $\mathrm{Mg}$ and $\mathrm{MgH}_{2}$ at a selected position within the acquired SI pattern. The volume fraction is given by the ratio of the individual plasmon contribution $A_{M g}^{\text {plasmon }}$ and $A_{M g H 2}^{\text {plasmon }}$ to the superposition of both $A_{\text {super }}^{\text {plasmon }}=A_{M g}^{\text {plasmon }}+A_{M g H 2}^{\text {plasmon }}$ :

The EEL spectra were evaluated in the following way using "DigitalMicrograph ${ }^{\circledR}$ " (version 2.32.888.0) from Gatan, Inc.: The individual low-loss spectra were deconvolved with the "Fourier-Log" algorithm! A single scattering contribution of the plasmon regions results from this procedure. Subsequently, "MLLS Fitting" was used to describe the obtained single scattering contributions. For the MLLS fitting, two reference spectra were chosen, one from the $\mathrm{Mg}$ film and one from a fully hydrided part.

\subsection{X-ray diffraction techniques}

The following chapter introduces the X-ray techniques utilized in this work. For details on different X-ray techniques see e.g. "Moderne Röntgenbeugung: Röntgendiffraktometrie für Materialwissenschaftler, Physiker und Chemiker" by Spiess et al. 215. The techniques applied in this work can be divided into two kinds. First the grain size and structure was analyzed before and after hydrogen loading by Xray diffraction (XRD). This allowed a comparison with results of the TEM studies presented above (see section 3.4). Additionally, some samples have been studied at two synchrotron sources: at the Deutsches Elektronen-Synchrotron (DESY), beamline PETRA P08, Hamburg, and at the European Synchrotron Radiation Facility (ESRF), beamline BM20, Grenoble. These measurements allowed to study the XRD peak change in the samples during hydrogenation.

\footnotetext{
${ }^{4}$ Here, the zero-loss was described by the "reflected tail" model and the reconvolution model was chosen with the "zero-loss modifier".
} 


\subsubsection{Analysis of grain structure and grain size}

Grain structure information can be evaluated from the XRD measurements by Bragg's law [216]:

$$
m \cdot \lambda=2 \cdot l_{h k l} \sin \left(\frac{2 \Theta_{h k l}}{2}\right)
$$

$m$ is the diffraction order, $\lambda$ the x-ray wavelength, $l_{h k l}$ the distance between two (hkl) crystal planes and $\Theta_{h k l}$ the Bragg angle. Bragg's law describes the diffraction of x-rays on the (hkl) crystal planes of the sample depending on the incident angle of the x-ray's. If Bragg's law is fulfilled the x-rays constructively interfere with each other resulting in an intensity maximum. This means that by varying the angle of the x-rays maximal intensities are measured at positions that correlate to the out-of-plane lattice planes found in the thin films studied in this work.

A second grain size value, as comparison with the value evaluated from TEM studies, can be approximated using Scherrer's formula [217]:

$$
B_{\text {size }}(2 \Theta)=\frac{\kappa \lambda}{d \cos (2 \Theta / 2)}
$$

It describes how the peak broadness $B$ of a diffraction peak increases with decreasing crystallite size $d . \lambda$ is the wavelength of the x-rays, $\Theta$ the angle of the peak position and $\kappa \approx 1$ the so called Scherrer constant. $\kappa$ can vary between 0.62 to 2.08 . For the materials in this work a value of $0.9 \pm 0.2$ seems reasonable [218. Additional effects lead to a broadening of XRD peaks, namely an instrument broadening $B_{\text {inst }}$, because of non-ideal beam optics and non-uniform strains $B_{\text {strain }}$. Uniform strains lead to a general shift of the peak from its expected Bragg position. If the XRD peaks have Lorentzian shape the overall broadness $B$ can be expressed as [219]:

$$
B=B_{\text {size }}+B_{\text {strain }}+B_{\text {inst }} .
$$

For this work the strain broadening in the films is assumed to be relatively small compared to the size broadening leading to $B_{\text {size }} \approx B_{\text {size }}+B_{\text {strain }}$. The instrument broadening was tried to be corrected by taking the broadness of the silicon substrate peaks and subtracting them from the film peak broadness. On the one hand the substrate peak should not have a significant size or strain influence on the broadness as the substrates are single crystals. On the other hand the instrument broadening is angle dependent, therefore the instrument broadening is not expected to be equal at the positions of the silicon substrate peak and the thin film peaks. However, as the instrument broadening is not known over the whole angle range, this correction is the best possible for this work.

The analysis of the grain structure and grain size was done using a Bruker D8 Discovery high resolution diffractometer. It is equipped with a Siemens KFL $\mathrm{Cu}$ 


\section{Applied experimental methods and developed algorithms}

$2 \mathrm{~K}$ source, operated at $40 \mathrm{kV}$ and $40 \mathrm{~mA}$. The X-ray beam is parallelized by a $1 \mathrm{D}$ Goebel mirror, which filters the $\mathrm{Cu} K_{\beta}$ lines (wavelength $\lambda=1.392 \AA$ ). This leaves the $K_{\alpha_{1}}$ line at a wavelength of $\lambda=1.541 \AA$ and the $K_{\alpha_{2}}$ line at a wavelength of $\lambda=1.544 \AA$ [220]. The two lines have an intensity ratio of $I_{K_{\alpha_{1}}} / I_{K_{\alpha_{2}}} \approx 2$. The $\mathrm{X}$-ray intensity is measured by a $1 \mathrm{D}$ Lynxeye detector with an angle resolution of $\Delta(2 \Theta)=0.01^{\circ}$. For the measurements a $0.1 \mathrm{~mm}$ slit and a $4 \mathrm{~mm}$ mask were used on the primary side in front of the sample. On the secondary side an automated slit is fixed in the setup, which was opened completely (resulting in an opening of $9 \mathrm{~mm}$ ). The detector was operated in the 1D mode, using all 192 detector channels simultaneously.

\subsubsection{XRD: in situ hydrogen loading}

Samples have been studied at two different synchrotron radiation sources. At the Deutsches Elektronen-Synchrotron (DESY), beamline PETRA P08, Hamburg a wavelength of $\lambda=0.9998 \AA$ was used. The samples were loaded with a loading setup similar to the one presented above (section 3.2.1) with a Kapton window to allow for synchrotron radiation to pass into and out of the sample chamber. In contrary to the setup presented above, the volume of the reservoir and sample chamber is not known for this setup, but the volume is much larger. Therefore, the conditions for diffusion from a limited volume do not apply (compare section 3.2.2). The pressure does not change after beginning the loading procedure, indicating that the reservoir is large enough to assume a constant surface concentration. The solution of Fick's law for these condition is given by equation 3.7. A four-point resistance measurement was carried out during hydrogen loading.

At the European Synchrotron Radiation Facility (ESRF), beamline BM20, Grenoble a similar measurement was done using a wavelength of $\lambda=1.078 \AA$. The setup employed at the ESRF was already presented by H. Uchida who also used it for in situ hydrogen loading experiments of $\mathrm{Mg}$ thin films [76]. The main difference to the other presented loading setups is that this one works with a constant hydrogen flow, artificially holding a constant pressure at the sample surface. This is an ideal situation for the solution given by equation 3.7. However, it takes up to $2 \mathrm{~h}$ to reach the highest measured pressure of $8 \cdot 10^{5} \mathrm{~Pa}$, because the hydrogen is limited by a flow controller. During this time the concentration can only be assumed to be constant in a short enough time frame. The setup does also include a four-point resistance measurement and offers the possibility to heat the sample.

The measurements at both beamlines allow to measure the development of one Bragg peak during hydride formation over time. This allows measuring the XRD peak area $A^{\text {peak }}$ over time. The XRD peak area is proportional to the volume of the material in the sample [215]. Assuming the model of Uchida et al. [44] the $\mathrm{MgH}_{2}$ 
grows as a (after some time) closed layer starting from the top of the $\mathrm{Mg}$ thin film. Therefore, the volume of $\mathrm{Mg}$ and $\mathrm{MgH}_{2}$ in the thin films can be simplified to only the layer thickness of the magnesium $d_{M g}$ and magnesiumdihydride $d_{M g H 2}$ layer:

$$
\begin{aligned}
d_{M g H 2} & =d_{0}-d_{M g} \\
\Leftrightarrow d_{M g H 2} & =d_{0}-d_{0} \frac{A_{M g}^{\text {peak }}}{A_{0}^{\text {peak }}} \\
\Leftrightarrow \frac{d_{M g H 2}}{d_{0}} & =1-\frac{A_{M g}^{\text {peak }}}{A_{0}^{\text {peak }}} .
\end{aligned}
$$

$A_{0}^{\text {peak }}$ is the $\mathrm{Mg}$ peak area before loading where $d_{0}=d_{M g}$. Assuming that the hydride formation is diffusion limited and the diffusion process is one-dimensional, the hydride thickness over time can be described as $d_{M g H 2}=\sqrt{2 D_{M g H 2}} \cdot \sqrt{t} \cdot D_{M g H 2}$ is the diffusion coefficient of hydrogen diffusing through $\mathrm{MgH}_{2}$. This finally leads to a description of the peak area over time:

$$
\frac{A_{M g}^{\text {peak }}}{A_{0}^{\text {peak }}}=1-\frac{\sqrt{2 D_{M g H 2}}}{d_{0}} \cdot \sqrt{t} .
$$

Plotting the normalized peak area $A_{M g}^{\text {peak }} / A_{0}^{\text {peak }}$ over the square root of time results in the diffusion constant of hydrogen in magnesiumdihydride as the slope of a straight line with a $y$-intersect of one. If the data points do not form a straight line in this plot it can be seen as an indication that the hydride formation is not limited by a one-dimensional diffusion process.

\subsection{Finite-element simulations}

In the following section finite-element-simulations (FEM simulations) performed for this work are introduced. These are employed to gain additional insight into how the grain boundary diffusion and grain diffusion contribute to the overall diffusion in the sample. The experimental techniques introduced above only allow to measure the overall diffusion coefficient of hydrogen in the sample. A comparison with FEM simulations may help to separate the influence of the single components. Finiteelement simulations divide a given geometry by means of a mesh into subdivisions. For each of the mesh nodes the relevant differential equations are solved (e.g.the diffusion equations as given by equation 2.10 and equation 2.11). By combining the equations of each subdivision into a larger system the behavior of the overall geometry can be calculated. In this work, the FEM simulations were performed using the commercial software COMSOL Multiphysics ${ }^{\circledR}$ Modeling Software, version 5.2. The first part introduces the geometries modeled as well as the meshing used. In 
addition the stability of the simulations is tested in dependence on the mesh. In the second section the data evaluation of the simulation is discussed. The experimental procedure and the results are published in "FEM simulation supported evaluation of a hydrogen grain boundary diffusion coefficient in $\mathrm{MgH}_{2} "$ [221].

\subsubsection{Simulation setup}

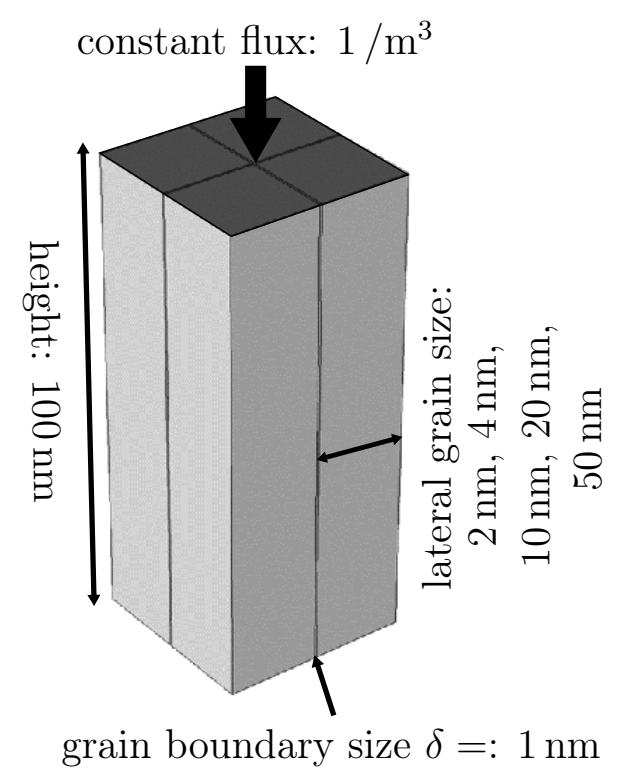

(a) Schematic drawing of the FEM simulation

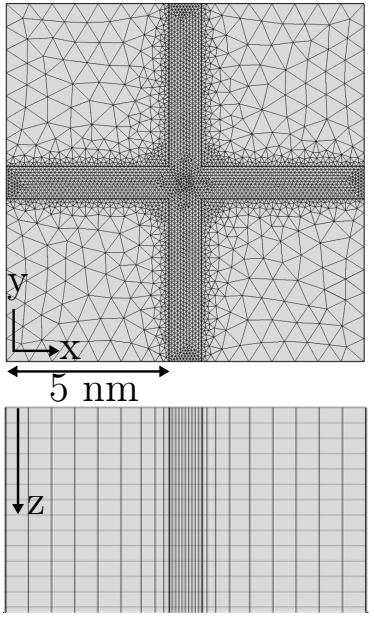

(b) Meshing of the FEM simulation

Figure 3.12.: Geometry (a) and mesh(b) used with the COMSOL Multiphysics ${ }^{\circledR}$ Modeling Software, version 5.2. The geometry employs periodic boundary conditions on the sides, resulting in an infinite $100 \mathrm{~nm}$ thin film. The mesh was set on the surface, refined and swept in the diffusion direction (z-direction). The figure is reproduced from [221].

The 3-D Finite-Element simulations were performed using the "Transport of diluted Species" physics module. This models the transport of a single species of low concentrations. This seems to be well applicable as hydrogen has low solubility in magnesium and magnesiumhydride $\left(\mathrm{MgH}_{2}\right.$ being a stoichiometric phase). For this work, convection, as an additional transport mechanism, was deactivated. Figure 3.12 shows the modeled geometry. The geometry is build up out of $100 \mathrm{~nm}$ high grains that are separated by $1 \mathrm{~nm}$ thick slabs of grain boundaries. Periodic boundary conditions are applied on the outside of the grains so that the overall geometry models an infinite $100 \mathrm{~nm}$ thin film with columnar, box-shaped grains. Five different grain sizes $d$ of $2 \mathrm{~nm}, 4 \mathrm{~nm}, 10 \mathrm{~nm}, 20 \mathrm{~nm}$ and $50 \mathrm{~nm}$ were studied. On top of this, a constant flux $J$ of hydrogen of $J=1 \mathrm{~m}^{-3}$ is applied. The diffusion coefficient of the grain boundary was set to $D_{G B}=10^{-17} \mathrm{~m}^{2} / \mathrm{s}$, as a rough mean value of the different literature values at room temperature (compare figure 2.6). The diffusion 
coefficient of the grain was varied, starting at $D_{V}=10^{-18} \mathrm{~m}^{2} \mathrm{~s}^{-1}$ and decreasing in orders of magnitude up to $D_{V}=10^{-22} \mathrm{~m}^{2} \mathrm{~s}^{-1}$. This contains the assumption done by Fisher and others after him, that the grain boundary diffusion is faster than the grain diffusion (compare section 2.2.3). The initial concentration $c_{H}$ in the whole sample was $c_{H}=0 / \mathrm{m}^{3}$. The simulation gives the concentration in arbitrary units of $1 / \mathrm{m}^{3}$ at chosen time levels of $t=10^{-2} \mathrm{~s}, 10^{-1} \mathrm{~s}, 1 \mathrm{~s}, 10 \mathrm{~s}$ and $100 \mathrm{~s}$. The simulation times and simulated lengths were adjusted to the modeled diffusion coefficients, but the results of the simulation can easily be transferred to other diffusion coefficients. The same is true for different time and length scales. $D \approx l^{2} / t$ allows recalculating the diffusion coefficient $D$, time $t$ and distance $l$ in relation to each other. An example of the meshing is shown in figure 3.12. For all grain sizes a free triangular mesh was applied on the top plane (lying in $\mathrm{x}$-y-direction) of the geometry which was swept in the direction of diffusion (z-direction). To control the influence of the meshing on the results the concentration in the grains, in the grain boundaries and in some random cut planes, containing both, were studied. The mesh was refined until the relative change in the average concentration was below $0.2 \%$ between two refinement steps for all regions and for all times. The resulting meshing was used in the final simulations. In z-direction this was always the case for a division into 75 or more elements. The number of elements and their size of the free triangular mesh in $\mathrm{x}$ - and $\mathrm{y}$-direction was different for the different grain sizes. The total number of elements increased from 231600 elements for a grain size of $2 \mathrm{~nm}$ to 839550 for a grain size of $50 \mathrm{~nm}$.

\subsubsection{Data evaluation}

To evaluate the concentration data depending on time and space, the geometry was divided into cut-planes parallel to the entrance surface. The average concentration $\bar{c}$ in these cut-planes was determined thereafter, resulting in an average concentration for a given diffusion length from the surface and for a given time. This resembles many experimental procedures used to evaluate the diffusion coefficient in systems [51. The resulting concentration-to-depth dataset can be evaluated using the analytical solution of the three Harrison's regimes, as introduced in section 2.2.4. Figure 3.13 exemplifies one dataset. It gives the result of the simulation for a grain size of $d=2 \mathrm{~nm}$, a volume diffusion coefficient of $D_{V}=10^{-20} \mathrm{~m}^{2} / \mathrm{s}$ and a time $t=$ 100 s. Also plotted are the analytical solutions of all three Harrison regimes with the same input values, a grain boundary size of $\delta=1 \mathrm{~nm}$ and a grain boundary diffusion coefficient of $D_{G B}=10^{-17} \mathrm{~m}^{2} / \mathrm{s}$. One can see that none of these curves fit the data perfectly. While this already qualitatively shows that the solutions for the different regimes represent the data differently well, they do not give a quantitative result. Here, the analytical solutions of the three regimes are fitted to the data and an artificial grain boundary diffusion coefficient is extracted, called $D_{G B}^{e x t}$. 


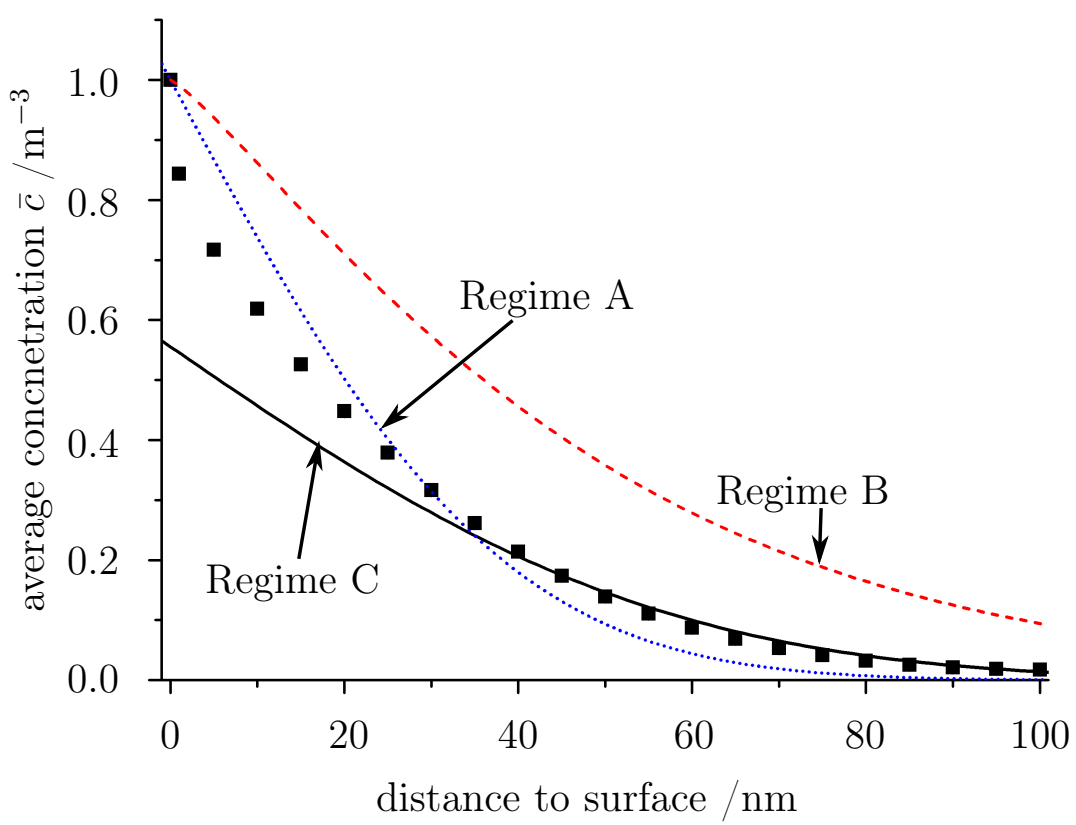

Figure 3.13.: Example of a data set taken from the Comsol Multiphysik simulation. The data was taken for a grain size of $d=2 \mathrm{~nm}$, a grain diffusion coefficient of $D_{V}=10^{-20} \mathrm{~m}^{2} \mathrm{~s}^{-1}$ and a time $t=100 \mathrm{~s}$. Also plotted are the analytical solutions of all Harrison regimes. Regime A's solution is given by the dotted blue line, regime B by the dashed red line, while regime C's solution is the solid black line. No solution fits the data perfectly, but for this case the solution of regime A fits best, while the solution for regime $\mathrm{C}$ still fits better than the solution of regime B. The figure is reproduced from M. Hamm et al. [221].

The exact process for extracting $D_{G B}^{e x t}$ is different for each Harrison regime. For regime $\mathbf{A}$, the solution for a constant source leaking into a semi-infinite medium is applied [201]:

$$
\bar{c}=c_{0}\left(1-\operatorname{erf}\left(\frac{z}{4 D t}\right)\right) .
$$

$c_{0}$ is the starting concentration at $t=0, z$ is the distance from the surface. $D$ is the relevant diffusion coefficient for the process. For regime A this is $D_{\text {eff }}$. Equation 2.24 gives the appropriate value for $D_{\text {eff }}$ for columnar grains as studied in this simulation. Equation 2.24 simplifies for the FEM simulations as the simulations do not include any segregation $(s=1)$, therefore $D_{\text {eff }}=\phi_{G B} D_{G B}+\left(1-\phi_{G B}\right) D_{V}$. If regime $\mathrm{A}$ is assumed, $D_{G B}^{e x t}$ can be extracted from the simulated data by fitting equation 3.21 to the data. This gives a value $D \stackrel{!}{=} D_{\text {eff }}$ and $D_{G B}$ can be calculated as $D_{V}$ is known in a given simulation and $\phi_{G B}$ can be calculated for the simple geometry in use here:

$$
\phi_{G B}=1-\left(\frac{d^{2}}{(d+\delta)^{2}}\right)
$$


For regime $\mathbf{B} \ln (\bar{c})$ is plotted as function of $z^{6 / 5}$. This can be fitted by equation 2.21 and $D_{G B}^{e x t}$ can be calculated. For the simulations $s$ is again equal to one (compare equation 2.23), while $D_{V}$ and $t$ depend on the simulation parameters.

Regime $\mathbf{C}$ uses the same solution as regime A (equation 3.21), as both are a simple isotropic front. The difference is that for regime $\mathrm{C} D \stackrel{!}{=} D_{G B}^{e x t}$. This results from the fact that per definition in regime $\mathrm{C}$ only the grain boundaries transport material. Therefore, there is no contribution of the grain boundary volume fraction $\phi_{G B}$ in the solution.

Overall, by assuming one of the Harrison regimes a $D_{G B}^{e x t}$ can be calculated. This is similar to the procedure applied in an experiment. The advantage of the simulations is that the value $D_{G B}=10^{-17} \mathrm{~m}^{2} / \mathrm{s}$ fed into the simulations is known. Therefore, a comparison of the two allows evaluating under which conditions which regime is best used. A comparison is done by regarding the relative deviation $\varepsilon=\left(D_{G B}^{e x t}-10^{-17}\right) /\left(10^{-17)}\right)$ between the extracted and the fixed diffusion coefficients. A small $\varepsilon$ reflects a good description of the FEM simulation result by the analytical function of the chosen Harrisson regime.

In section 2.2.4 the additional regimes $\mathrm{B}_{1}$ to $\mathrm{B}_{4}$, as well as the varieties $\mathrm{A}^{\prime}, \mathrm{C}^{\prime}$ and $\mathrm{B}_{2}$ ' were discussed. $\mathrm{B}_{1}$ and $\mathrm{B}_{3}$ are not further discussed here as they are only transition states. For the FEM simulations, the varieties $\mathrm{A}^{\prime}, \mathrm{C}^{\prime}$ and $\mathrm{B}_{2}$ ' are equal to their normal regime because of the simple geometry studied. E.g. the effective grain boundary size $\delta^{e f f}$ is equal to the actual grain boundary size $\delta$. This leaves regime $B_{2}$, which is actually only regime $B$ as discussed before and regime $B_{4}$. As discussed in section 2.2.4 , regime $\mathrm{B}_{4}$ describes the case of isolated grain boundaries, but at the same time the bulk diffusion in z-direction is dominant. In this case the grain boundaries have no relevant influence on the diffusion process and no grain boundary diffusion coefficient can be extracted. Obviously, no analysis is possible under these conditions. In figure 2.11 the parameters analyzed in the simulations are indicated by a red box, showing which classes of polycrystals are researched. It can be seen that the grain sizes studied here mostly can be described as fine and ultrafine grained polycrystals. Only the largest grain sizes of $50 \mathrm{~nm}$ for small differences of the volume and grain boundary diffusion coefficient can be classified as a coarse grained polycrystals. 



\section{Structural characterization and individual discussion of $\mathrm{Mg}$ thin films upon hydrogen loading}

In some of Harrison regimes the grain size directly influences the overall diffusion. At the same time the grain size determines which Harrison regime is reached. Therefore, it is of importance to know the grain size and structure of the samples studied to determine the diffusion coefficient (see chapter 5). In the following, the results of the sample characterization will be presented. First, the initial state of the samples will be characterized. Afterwards, the changes during hydrogen loading will be shown. ETEM and XRD are valuable tools to study these changes in situ. The in situ ETEM results are published in "In situ hydrogen loading of magnesium thin-films observed with environmental TEM" 210]. Finally, the grain size and structure after hydrogen loading and unloading will be evaluated and compared with the initial results. An overview of all samples studied in this work can be found in table 4.1. It gives the sample name, the hydrogen loading pressures, present additives and what measurement methods were applied. Two sample preparation methods were employed to deposit thin films: ion beam sputtering (IBS) and pulsed laser deposition (PLD) (see chapter 3.1 for further information). Samples are labeled with IBS or PLD and an additional label, so the different samples can be directly distinguished.

\subsection{Initial film morphology}

The morphology of the thin films was studied by TEM and XRD as described in chapter 3.4 and chapter 3.5. An example of a typical XRD scan is given in figure 4.1. While the scan was measured on an IBS prepared film with a thickness of $4 \mu \mathrm{m}$, the results on the PLD samples are similar (additional XRD results can be found in appendix B.1.2). For all samples a (400) silicon diffraction peak is visible, originating from the substrate. The two $\alpha$ peaks and a $\beta$ peak to their left can be distinguished. In between some additional tungsten peaks can be found. They originate from aging of the X-ray source. Further visible XRD peaks are the (002) 


\begin{tabular}{|c|c|c|c|c|}
\hline Batch & Sample & $\begin{array}{l}\text { loading pressures } \\
/(\mathrm{hPa})\end{array}$ & used for... & Additives \\
\hline IBS-A & 1 & 800 & in situ XRD, TEM & $\begin{array}{l}\text { Fe (13 at\%), Cr (6 at\%), } \\
\text { Ni (1.5 at\%) }\end{array}$ \\
\hline IBS-A & 2 & 300 & in situ XRD & $\begin{array}{l}\text { Fe }(13 \text { at\%), Cr (6at\%), } \\
\text { Ni }(1.5 \text { at } \%)\end{array}$ \\
\hline IBS-A & 3 & 500 & $\begin{array}{l}\text { in situ XRD, TEM, } \\
\text { EDX }\end{array}$ & $\begin{array}{l}\text { Fe }(13 \text { at\% }), \text { Cr }(6 \text { at\% }) \\
\text { Ni }(1.5 \text { at\% })\end{array}$ \\
\hline IBS-A & 4 & not loaded & TEM , EDX & $\begin{array}{l}\text { Fe (13 at\%), Cr (6 at\%), } \\
\text { Ni (1.5 at\%) }\end{array}$ \\
\hline IBS-A & 5 & 300 & Gas volumetry & $\begin{array}{l}\text { Fe }(13 \text { at\%), Cr (6 at\%), } \\
\text { Ni (1.5 at\%) }\end{array}$ \\
\hline IBS-B & 1 & 50 & in situ XRD, TEM & $\begin{array}{l}\text { not verified (similar to } \\
\text { batch IBS-A) }\end{array}$ \\
\hline IBS-B & 2 & 800 & in situ XRD & $\begin{array}{l}\text { not verified (similar to } \\
\text { batch IBS-A) }\end{array}$ \\
\hline IBS-B & 3 & not loaded & TEM & $\begin{array}{l}\text { not verified (similar to } \\
\text { batch IBS-A) }\end{array}$ \\
\hline IBS-C & 1 & 300 & Gas volumetry, EDX & Fe $(1$ at $\%)$ \\
\hline IBS-C & 2 & 100 & Gas volumetry & Fe $(1 \mathrm{at} \%)$ \\
\hline IBS-D & 1 & $10,10,100$ & $\begin{array}{lr}\text { Gas volumetry, } \\
\text { Resistance mea- } \\
\text { surement, XRD, } \\
\text { TEM }\end{array}$ & $\mathrm{Fe}(2.5 \mathrm{at} \%)$ \\
\hline IBS-D & 2 & not loaded & EDX, XRD, TEM & $\mathrm{Fe}(2.5 \mathrm{at} \%)$ \\
\hline IBS-E & 1 & $10,10,100$ & $\begin{array}{l}\text { Gas volumetry, } \\
\text { Resistance mea- } \\
\text { surement, XRD, } \\
\text { TEM }\end{array}$ & $\begin{array}{l}\text { not verified (same } \\
\text { preparation conditions } \\
\text { as Batch IBS-D) }\end{array}$ \\
\hline IBS-E & 2 & not loaded & XRD, TEM & $\begin{array}{l}\text { not verified (similar to } \\
\text { batch IBS-D)) }\end{array}$ \\
\hline IBS-ET & EM & 6.5 & in situ TEM & $\begin{array}{l}\text { not verified (similar to } \\
\text { batch IBS-D) }\end{array}$ \\
\hline PLD-nc & & 7,7 & $\begin{array}{l}\text { Gas volumetry, } \\
\text { Resistance measure- } \\
\text { ment }\end{array}$ & no additives found \\
\hline \multicolumn{2}{|c|}{ PLD-Pd } & 10,25 & $\begin{array}{l}\text { Gas volumetry, } \\
\text { Resistance mea- } \\
\text { surement, XRD, } \\
\text { TEM }\end{array}$ & no additives found \\
\hline
\end{tabular}

Table 4.1.: Overview of the ion-beam sputter deposited samples (IBS) and pulsed laser deposited samples (PLD) studied in this work. The table gives the sample name and the hydrogen pressures applied to load the sample. In addition it gives the experimental methods used on each sample (more information about the methods are given in chapter 3.1). Finally, additives present in the samples are noted. 
magnesium peak and the weaker (004) peak, if the sample is thick enough. For thin films the (004) peak is too weak to be detected in the setup employed in this work. For the palladium capping layer two peaks are detected, one belonging to the (111) orientation and the other belonging to the (200) orientation. As said before the same peaks are detected for PLD prepared samples, however the magnesium peaks are generally weaker, which can be explained by a more nanocrystalline grain structure, as shown in the following.

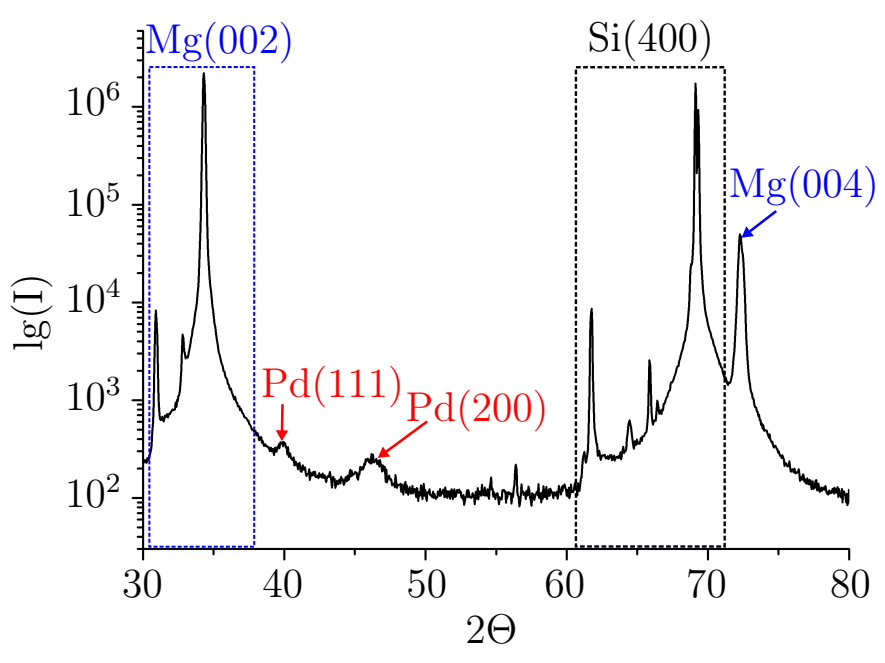

Figure 4.1: $\quad$ XRD diffractogram of a $4 \mu \mathrm{m}$ thick IBS film. For the magnesium layer only a $(002) /(004)$ out of plane orientation was found to form on the silicon substrate. The palladium capping layer shows a (111) and a (002) out of plane orientation. The same orientations were found for the PLD prepared samples.

The results of the TEM studies are summarized in figure 4.2. The figure shows two bright field and two dark field TEM images, representing the grain structures found for the IBS deposited and PLD samples. In addition, a sketch of the grain structure is given for each sample type. The ion beam sputtered samples show large columnar grains. Some smaller grains, still columnar in shape, can be found on the bottom of the film (meaning at the silicon substrate). These smaller grains have out-ofplane sizes of $100 \mathrm{~nm}$ to $200 \mathrm{~nm}$. However, most grains have out-of-plane sizes in the range of the film thickness. The average in-plane grain size for the small grains is generally smaller than for the large grains, but it is difficult to quantify because of low statistics. The in-plane grain size of all samples prepared by IBS are given in figure 4.3 in dependence on the film thickness. The error is the statistical variance of the measurements. It can be seen that the in-plane grain size seems to increase with increasing film thickness. This dependence is weak for the films studied in this work, because most films are of similar thickness. However, one much thicker film (IBS-C) was prepared, which shows larger in-plane grain sizes.

The pulsed laser deposited thin films show a different grain morphology. Most of the grains are nanocrystalline and equiaxed with grain sizes of $(11 \pm 5) \mathrm{nm}$. However, some large grains are found. The TEM image in figure 4.2 shows one of these large 

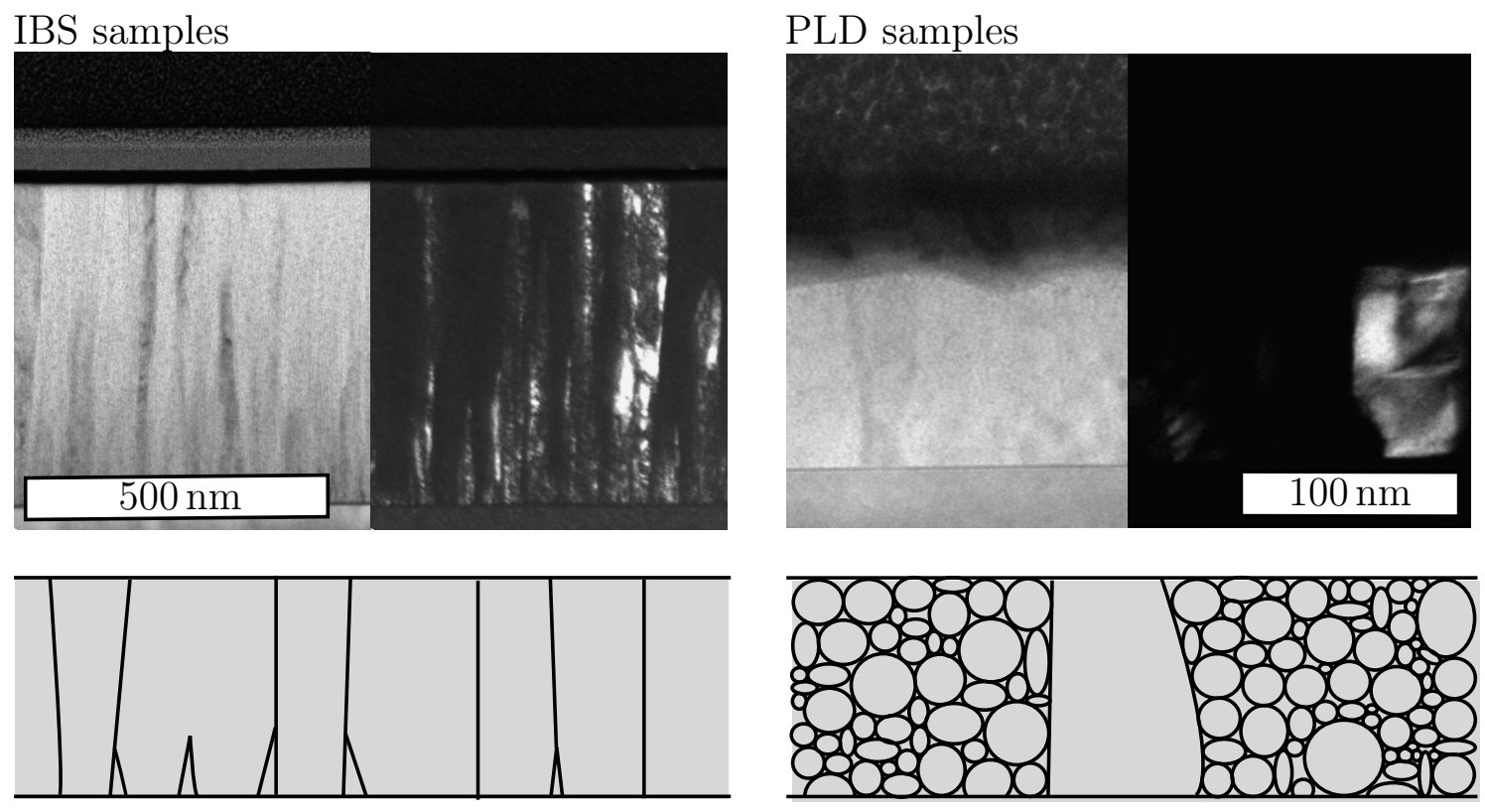

Figure 4.2.: Example of the grain morphology of the IBS samples (left) and the PLD samples (right). For each a bright field and dark field TEM image are shown. In addition, a schematic drawing of the typical grain morphology is shown below the TEM images.

Table 4.2.: Grain sizes of the thin magnesium films studied in this work. Before hydrogen loading the IBS samples have large, columnar grains and the grain size in-plane and out-of-plane is given separately. The PLD samples have some large grains before hydrogen loading. For these the out-of-plane and in-plane grain size is also given. However most grains before hydride formation are smaller and of equiaxed shape. Their grain size is given in a separate column. After hydride formation and decomposition all samples show approximately uniform equiaxed grains. The grain size was mostly studied in TEM cross sections, using dark field TEM. The values in brackets were measured using X-ray diffraction and Scherrer's formula.

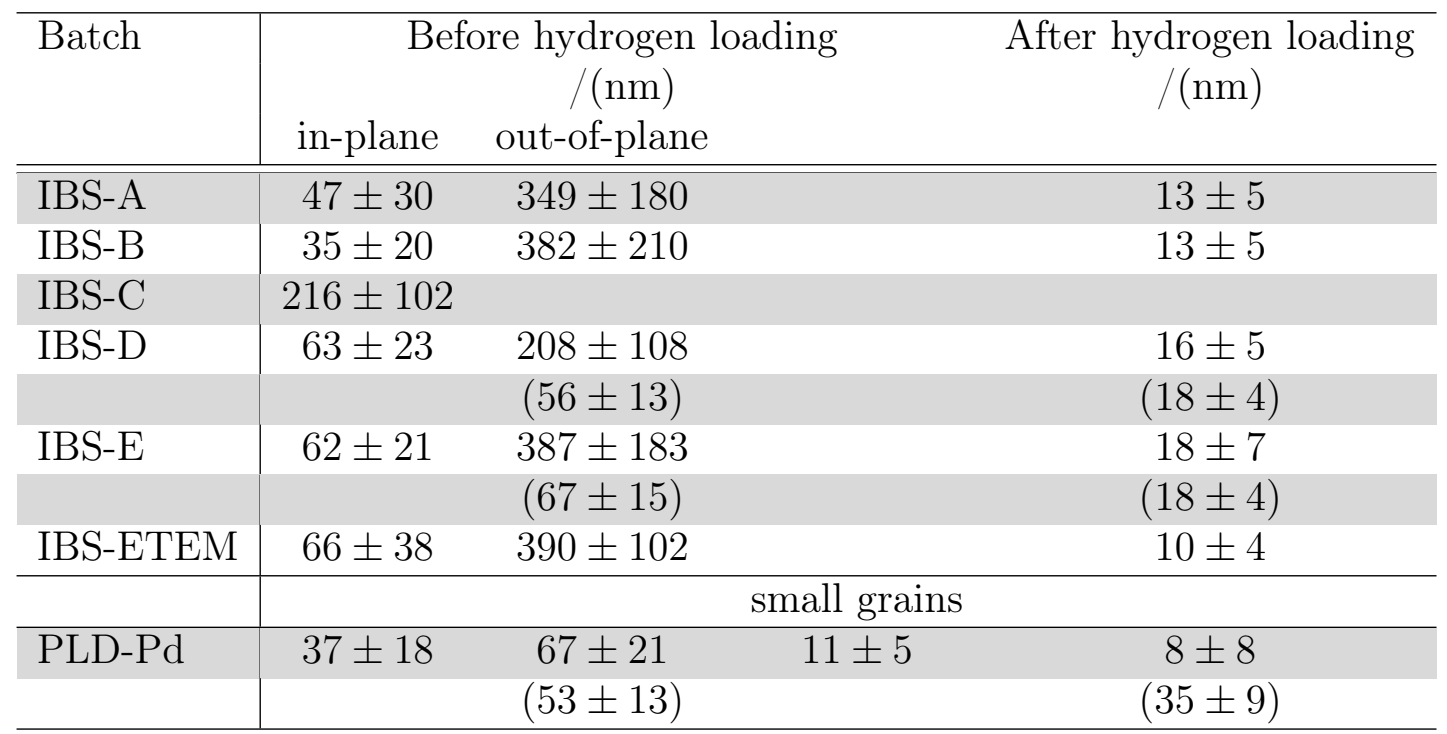


grains. Again the grain is roughly columnar in shape and the out-of plane size is equal to the film thickness. This is not always the case, some large grains do not cover the whole film thickness. These are similar to the smaller grains at the substrate interface in the IBS samples. The in-plane grain size of the large grains is similar to studied IBS films taking into account the film thickness. The film thicknesses of the PLD samples is between $100 \mathrm{~nm}$ to $200 \mathrm{~nm}$, so that the grain size does follow the same dependence shown in figure 4.3 .

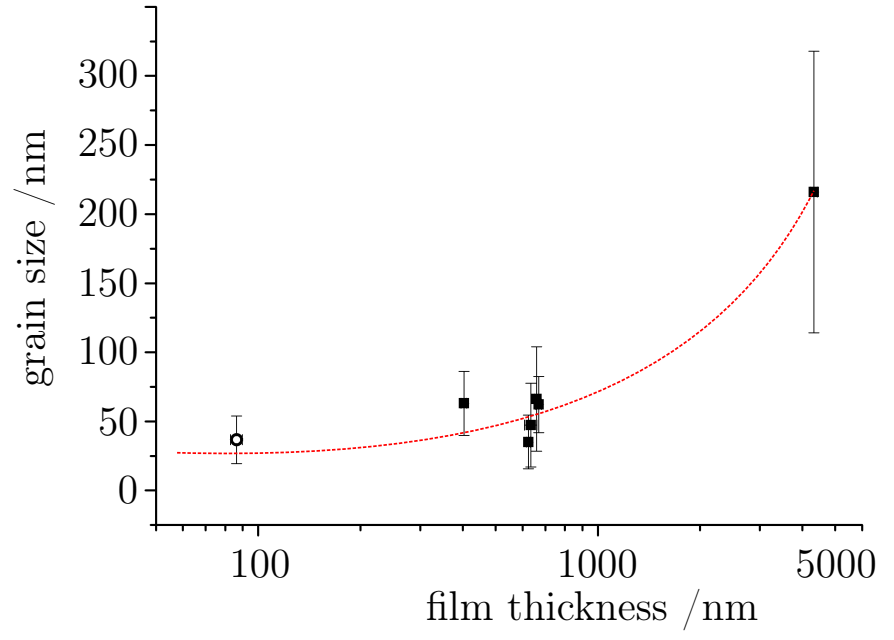

Figure 4.3: In-plane grain size in IBS and PLD prepared samples with increasing film thickness. Black squares belong to IBS films. Open black circles belong to the large, columnar grains in PLD films. The small equiaxed grains of the PLD films are not included.

The grain size of the different samples was evaluated from dark field TEM and, in some cases, by using Scherrer's formula (equation 3.19 in chapter 3.5). The results are collected in table 4.2. The grain sizes of samples that were loaded with hydrogen are also included and will be described in section 4.3 . For the unloaded samples there is a significant difference between the out-of-plane grain sizes evaluated by TEM and XRD 1 . The XRD grain sizes are smaller than the TEM values. However, it should be kept in mind that XRD gives an average of the whole illuminated volume of the sample, which is the illuminated surface area in the range of $10^{3} \mu \mathrm{m}^{2}$ multiplied by the film thickness. The TEM cross sections cover a much smaller volume fraction. In addition, the grain size evaluation by TEM may overestimate the number of large grains, because they are more noticeable in dark field TEM images (see figure 4.2). This may explain the larger out-of-plane average grain sizes measured by TEM for the IBS samples. The smaller grains at the substrate interface may reduce the average value measured by XRD more strongly than the average value evaluated by TEM. For the PLD samples XRD gives an average grain size between the values for small and large grains, which is why the XRD value are between the two values of $67 \mathrm{~nm}$ and $11 \mathrm{~nm}$ evaluated by TEM.

\footnotetext{
${ }^{1}$ XRD measurements only allow to evaluate the out-of-plane grain size, only this value can be compared.
} 


\subsection{Changes during hydrogen loading}

To study the hydride formation in situ a sample was loaded with hydrogen using a ETEM. A cross section lamella is not directly comparable to a thin film as it is exposed to different boundary conditions. Yet, it allows immediate insight into the process. The influences of these boundary conditions will be discussed in chapter 6.1 .2 taking into account the results of this section.

Figure 4.4: Relative thickness in the $\mathrm{MgH}_{2}$ for different positions measured by EELS. An EELS plasmon color map is shown for the $\mathrm{Mg}-\mathrm{MgH}_{2}$ thin film. Magnesium is colored in green and magnesiumdihydride in blue. The red plot below gives the relative thickness $d_{\text {lam }} / \lambda$ of the magnesiumdihydride. $d_{\text {lam }}$ is the thickness of the lamella in beam direction and $\lambda$ the mean free path of the electrons. The figure is reproduced from M.Hamm et al. [210].



Figure 4.4 shows the lamella studied at the end of the loading experiment. Two thinned windows (window 1 and window 2) are shown, which will be shown in more detail again below. An EELS plasmon map shows the distribution of $\mathrm{MgH}_{2}$ and $\mathrm{Mg}$ in the lamella at the end of the loading experiment. At the interface between magnesium and palladium the whole magnesium is hydrided. In the direction of the silicon substrate metallic magnesium can still be found. In addition, figure 4.4 shows the relative thickness $d_{\text {lam }} / \lambda$ of the lamella in the $\mathrm{MgH}_{2}$ region (marked by a red box). $d_{\text {lam }}$ is the thickness of the lamella in the beam direction, while $\lambda$ is the mean free path of the electrons in magnesiumdihydride. As the lamella is hydrided completely in the scanned region it allows comparing the relative thickness along the lamella. Window 1 was found to be thinner than window 2 and both are thinner than the less thinned regions between them.

\section{Nucleation and growth of $\mathrm{MgH}_{2}$ in a TEM lamella}

Figure 4.5 shows subsequent time steps of hydride formation in the lamella. After introducing the hydrogen into the chamber it takes some time until the first hy- 


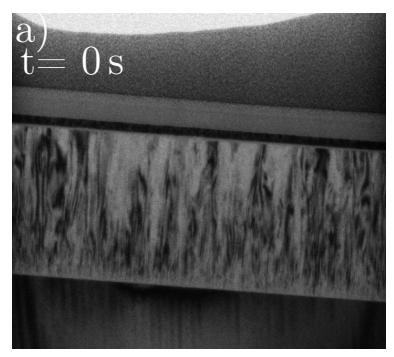

f)

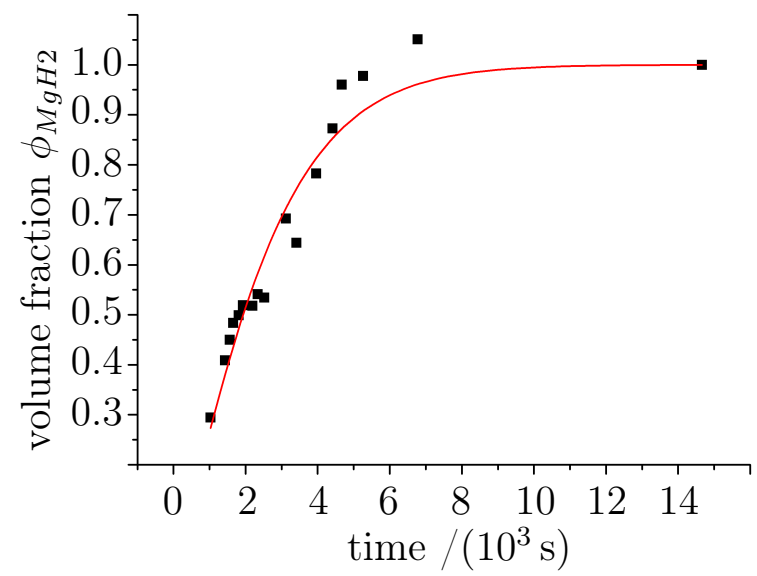

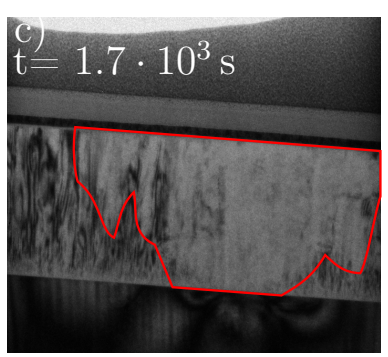
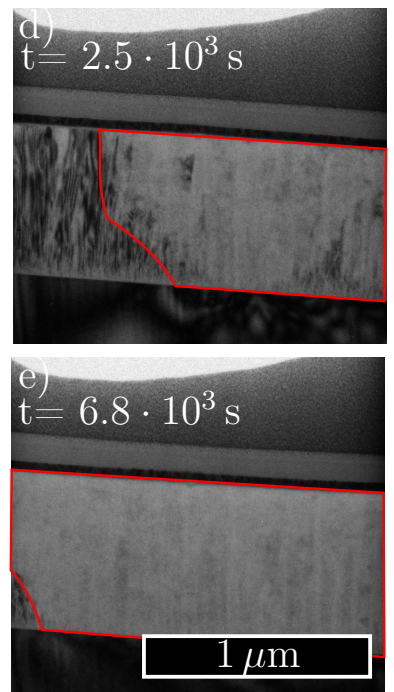

Figure 4.5.: Development of the magnesiumhydride after nucleation. The TEM images show the growth of the magnesiumhydride over time (the hydride is framed in red). At $t=0 \mathrm{~s}$ no hydride is formed yet. Afterwards, The hydride forms between images through the whole thickness of the magnesium thin film and grows further outwards afterwards. Also shown is the evaluated volume fraction of the hydride $\phi_{M g H 2}$ over time. The red line is the fit of equation 2.30. It gives a impingement parameter of $\eta=5 \cdot 10^{11}$, meaning that an evaluation after the JMAK model is appropriate. The results are discussed in the text. The figure is reproduced from M.Hamm et al. [210].

dride (marked in red) is detected. Afterwards, the first hydride forms very fast in window 1 between two subsequent images (in about $15 \mathrm{~min}$, see figure 4.5 a) and b) ). The first hydride directly forms throughout the whole thickness of the sample. Afterwards, it grows outwards in an almost half-spherical shape as shown in figure 4.5 a) to e). Figure $4.5 \mathrm{f}$ ) shows the volume fraction of the hydride evaluated from the visible change in grain structure. It was found that the hydride forms smaller nanocrystalline grains which lead to a different contrast in the TEM images, especially in dark field images. The volume fraction $\phi_{M g H 2}$ can therefore be evaluated as a function of time. The volume fraction $\phi_{M g H 2}$ follows equation 2.30 with an impingement parameter of $\eta=5 \cdot 10^{11}$, allowing to evaluate the data after the JMAK model. A JMAK exponent of 1.3 was found. This can be the result of two different processes. On the one side, for instantaneous nucleation it can be explained by a diffusion controlled kinetics with a dimension between two and three. 
On the other side, for a decreasing nucleation rate the process would be limited by one-dimensional diffusion. The images already show that the dimension is between two and three, as a growth in two dimensions can be seen with the thickness of the lamella being the third. The lamella is thinner than the $\mathrm{Mg}$ film is thick, which is why the hydride grows predominantly in two dimensions. An evaluation by Jander's model also gives a dimension $d \approx 3$. This confirms that the hydride is, after instantaneous nucleation, limited by the diffusion in more than one dimensions.

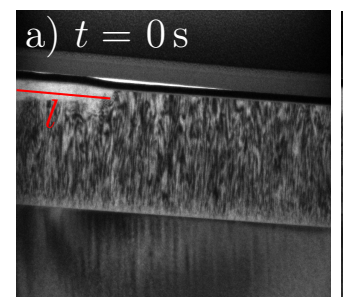

e)

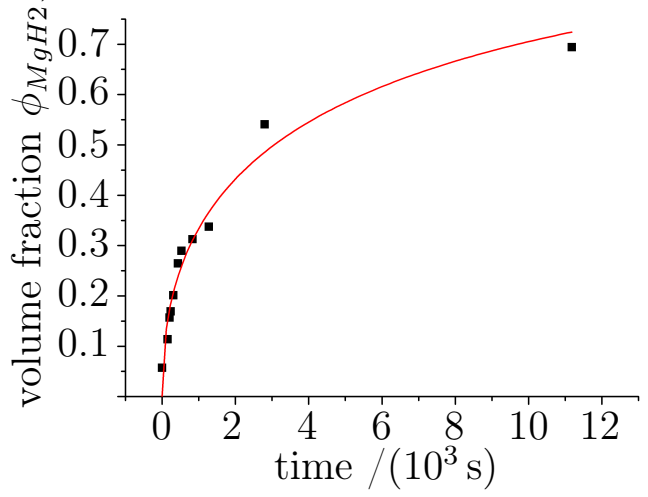

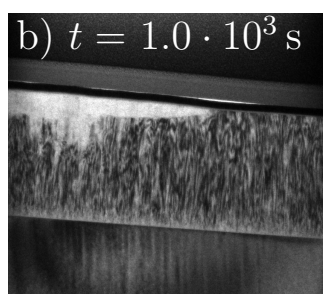

f)

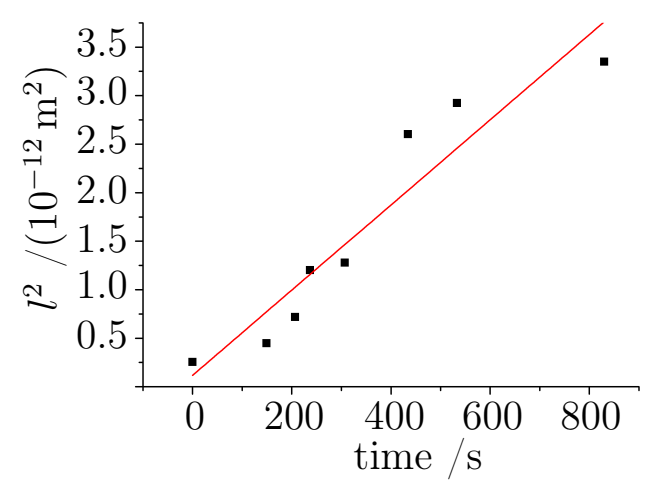

Figure 4.6.: Growth of the magnesiumdihydride along the palladium-magnesium interface after initial nucleation. The hydride initially nucleated left of the shown TEM images (compare figure 4.5p. Afterwards a hydride "finger" first grows along the palladium-magnesium interface before the hydride growth further into the magnesium thin film. Figure e) gives the evaluated hydride volume fraction $\phi_{\mathrm{MgH} 2}$ over time. The red line is the fit of equation 2.30. The results are discussed in the text. Figure f) gives the square length of the hydride along the interface $l$ over time (the length is shown exemplary in the first TEM image). The figure is reproduced from M.Hamm et al. [210].

After window 1 has started to hydride (compare figure 4.5) two "fingers" can be found at the interface to the palladium layer which grow outwards from the hydride in direction of window 2. One of these fingers is shown in figure 4.6 for the region of window 2 (see figure 4.4). The hydride first grows along the interface, covering it. On a much slower time scale it grows into the depth of the $\mathrm{Mg}$ film, a process that is not finished at the end of the experiment. Figure 4.6 a) again shows the volume fraction of the hydride $\phi_{M g H 2}$ plotted as function of time. The volume fraction here was evaluated until the whole interface was covered. The impingement factor is $\eta=4 \cdot 10^{10}$, again allowing to use the JMAK model. The JMAK exponent was found to be $n=0.5$, which corresponds to a diffusion limited growth with instantaneous nucleation in one dimension. This result was also confirmed in comparison with the 
contracting volume and Jander's model. Figure $4.6 \mathrm{~b}$ ) shows the squared length of the finger $l^{2}$ plotted over time. It shows a linear dependence as expected for a diffusion limited process and a diffusion coefficient can be calculated as $D=$ $(2 \cdot d)^{-1} l^{2} / t \approx 2 \cdot 10^{-15} \mathrm{~m}^{2} \mathrm{~s}^{-1}$.

\section{Confirmation of $\mathrm{MgH}_{2}$ formation and decomposition in a TEM lamella by EELS}
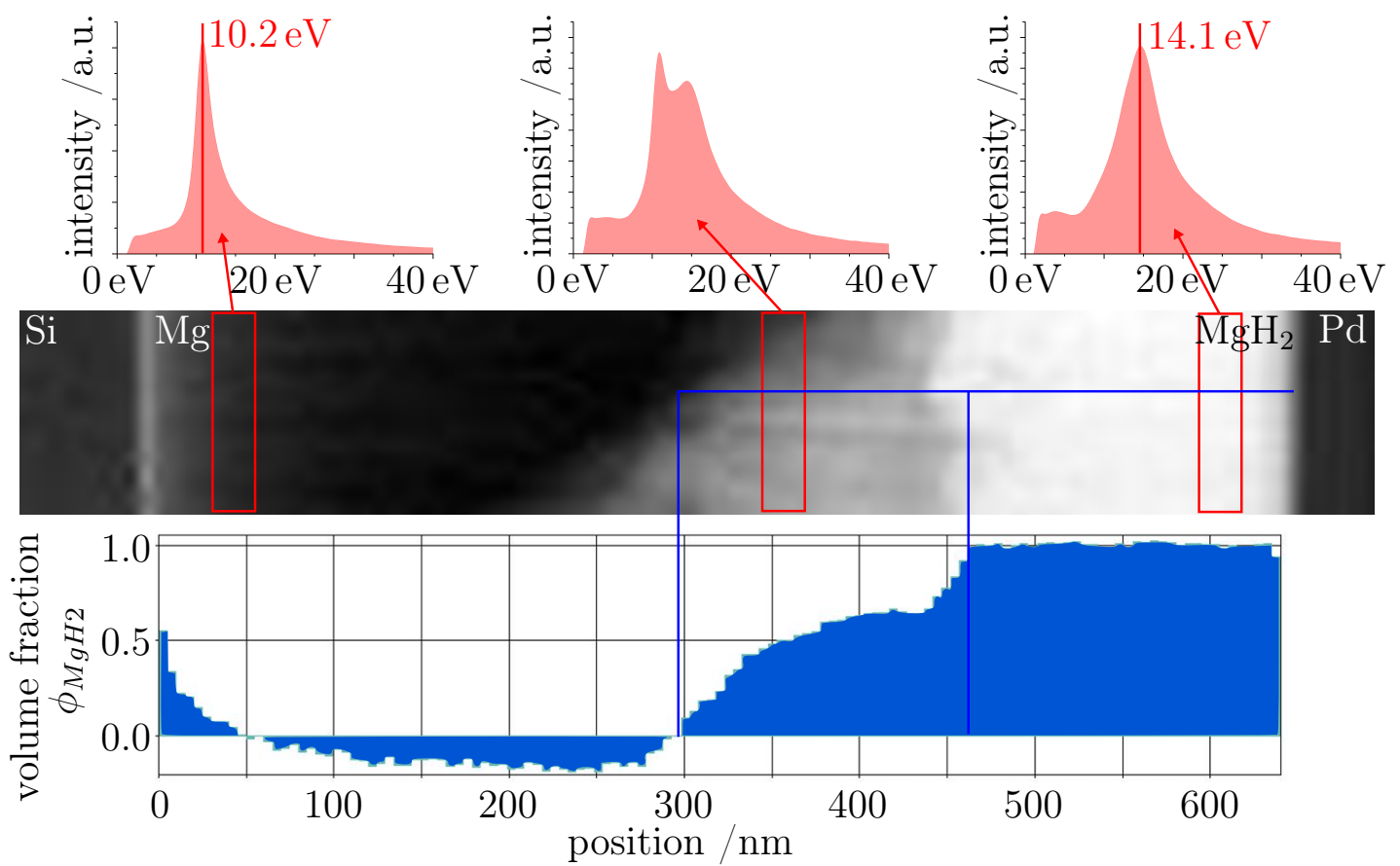

Figure 4.7.: Volume fraction of $\mathrm{MgH}_{2} \phi_{M g H 2}$ evaluated by EELS plasmon contribution depending on the position. Shown is a EELS map with part of the Si substrate on the left and the $\mathrm{Pd}$ capping layer on the right. Between them $\mathrm{Mg}$ and $\mathrm{MgH}_{2}$ can be found and distinguished by their plasmon peaks shown above the EELS scan. Where both materials are present the superposition of both peaks is found. Below the map the evaluated volume fraction $\phi_{M g H 2}$ is plotted for one line scan.

EELS allows verifying that the regions where the grain structure becomes nanocrystalline are the same regions where $\mathrm{MgH}_{2}$ is formed. Figure 4.7 shows an EELS mapping of the partially hydrided film. An EELS map was already shown in figure 4.4. Here, a more detailed example is given. For the pure magnesium film an EELS plasmon peak is found at $10.2 \mathrm{eV}$, which most probably corresponds to the $\mathrm{Mg}$ peak at $10.6 \mathrm{eV}$ known from the literature [212]. In regions where the grain structure changes to nanocrystalline a different plasmon peak is found at $14.1 \mathrm{eV}$. It corresponds well to the reported $\mathrm{MgH}_{2}$ peak at $14.6 \mathrm{eV}$ [212]. Using the two single peak spectra the volume fraction $\phi_{M g H 2}$ was evaluated in the mixed regions. The result 
of one line scan is shown below the EELS map in figure 4.7. It nicely correlates the formation of the hydride with the formation of a nanocrystalline structure.

Figure 4.8: TEM image and EELS map of sample IBS-ETEM after hydrogen loading and unloading by contact with air. While the changes in grain structure are irreversible, no hydride is left in the sample as confirmed by EELS. The figure is reproduced from M.Hamm et al. [210].

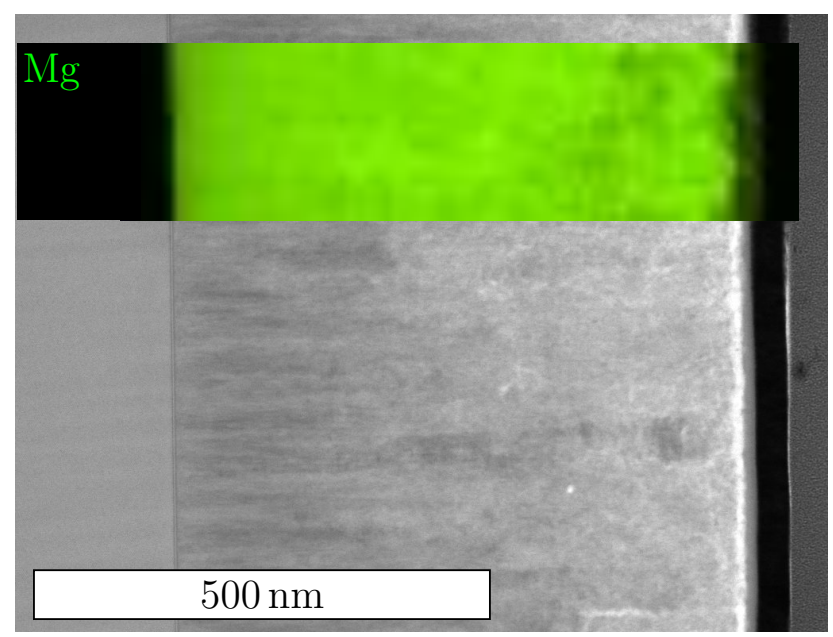

After the hydrogen loading experiment was stopped the lamella was removed from the TEM. After $5 \mathrm{~d}$ in air the lamella was once more studied in the TEM, this time without hydrogen. The result is shown in figure 4.8. The nanocrystalline structure stayed unchanged. However, the different structure was no longer correlated to magnesiumhydride. EELS mapping found no signs of the hydride, only of pure magnesium. This was true for nanocrystalline regions as well as regions with the original grain structure. This demonstrates two facts. First, while the formation of $\mathrm{MgH}_{2}$ leads to the formation of a nanocrystalline structure this structure is not reversible. It stays nanocrystalline after the hydrogen is removed. Second, the sample does unload at room temperature while in air. This is also confirmed for thin film samples by other measurements discussed in the following. Paik et al. reported that $\mathrm{MgH}_{2}$ transforms to $\mathrm{Mg}$ under the influence of the electron beam [212] in vacuum. In this experiment in hydrogen atmosphere this effect was not found.

\section{Comparison of experimental results of the $\mathrm{MgH}_{2}$ formation in the TEM lamella to results in thin film}

The behavior found in the TEM lamella and presented above can also be confirmed for thin films by other experimental methods. These will be presented in the following. It shows that the different boundary conditions in the lamella do not lead to a fundamentally different behavior.

Figure 4.9 shows the resistance measurement during the loading of sample IBSD1. The black curve shows the measured resistance, while the blue curve shows the volume fraction calculated by the three-layer model (see equation 3.14). The 


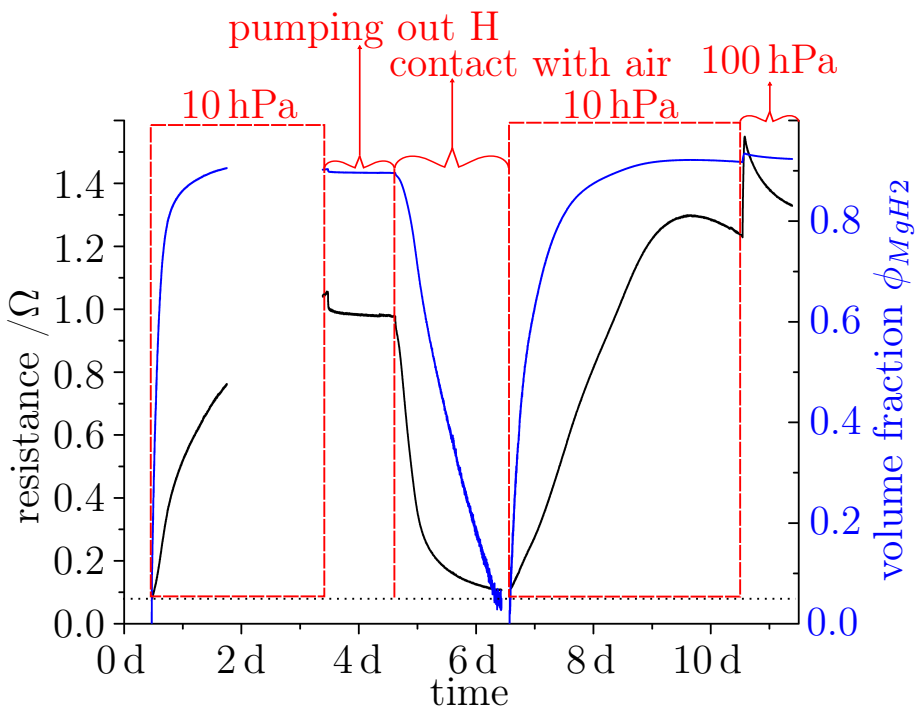

Figure 4.9: Black curve: development of resistance of a magnesium thin film during hydrogen loading. Blue curve: volume fraction of the magnesiumhydride calculated from the resistance measurement. The sample can be loaded, unloaded by contact with air and loaded again. The loading pressures are given above the corresponding loading period

sample was loaded at a hydrogen pressure of $10 \mathrm{hPa}$, thereafter the hydrogen was removed and air was introduced in the sample chamber. Afterwards, the loading process was repeated at $10 \mathrm{hPa}$ and a second loading step at $100 \mathrm{hPa}$ followed directly afterwards. During the loading steps at $10 \mathrm{hPa}$ the resistance increases. The volume fraction $\phi_{M g H 2}$ shows the formation of a hydride layer, which grows to a hydride volume fraction $\phi_{M g H 2}$ of more than 0.9. Removing the hydrogen atmosphere does lead to a slight drop in resistance, but the volume fraction $\phi_{M g H 2}$ does not show a relevant decrease. It needs the exposure to air to decrease the resistance and the $\mathrm{MgH}_{2}$ volume fraction $\phi_{M g H 2}$ to their initial values. This fits to the results of the in situ TEM study, which showed the vanishing of $\mathrm{MgH}_{2}$ after contact with air. The resistance does not drop back down to the original starting value, but remains at an increased value. This can be easily correlated to the added grain boundaries in the material after the formation of the nanocrystalline structure (see chapter 2.1.3 and citation [105]). The second loading at $10 \mathrm{hPa}$ behaves similar to the first. At its end a decrease in resistance is visible, which will be discussed in chapter 6.3.3. This decrease is almost not visible in $\phi_{\mathrm{MgH} 2}$.

The additional loading step at $100 \mathrm{hPa}$ shows a different behavior: a steep increase in the resistance which afterwards falls back down again on very long time scales. This process is not finished after several days. In the measurement of sample IBSE1 the same increase and decrease was found. Here, the decreased was measured for two month and still no equilibrium was reached. Again the increase and later decrease are visible in the resistance measurement but create no relevant change to the calculated volume fraction $\phi_{M g H 2}$. This indicates that it is not a change in the volume fraction of the magnesiumhydride but a secondary effect.

Figure 4.10 shows XRD peaks measured in situ at the ERSF in Grenoble, France. The (002) $\mathrm{Mg}$ peak and the (110) $\mathrm{MgH}_{2}$ peak were measured during loading with 
hydrogen. Before the loading starts no hydride peak is present. After the hydrogen gas is introduced, the $\mathrm{Mg}$ peak vanishes and the hydride peak appears. After no magnesium is left the hydride peak is still less intense than the original Mg peak. This fits to the nanocrystalline structure that forms. After the film was completely hydrided hydrogen was pumped out. The hydride stayed stable in vacuum as it did in the resistance measurement in figure 4.9. However, after heating the sample the hydride peak vanishes at temperatures between $75^{\circ} \mathrm{C}$ to $90^{\circ} \mathrm{C}$. Afterwards, higher intensity can be found at the $\mathrm{Mg}$ peak position but no clear peak. $\mathrm{Mg}$ peaks have been found in other samples that were loaded and afterwards unloaded by contact with air. The peak area was found to be much smaller after hydrogen loading, indicating the nanocrystalline structure found in the TEM experiments.
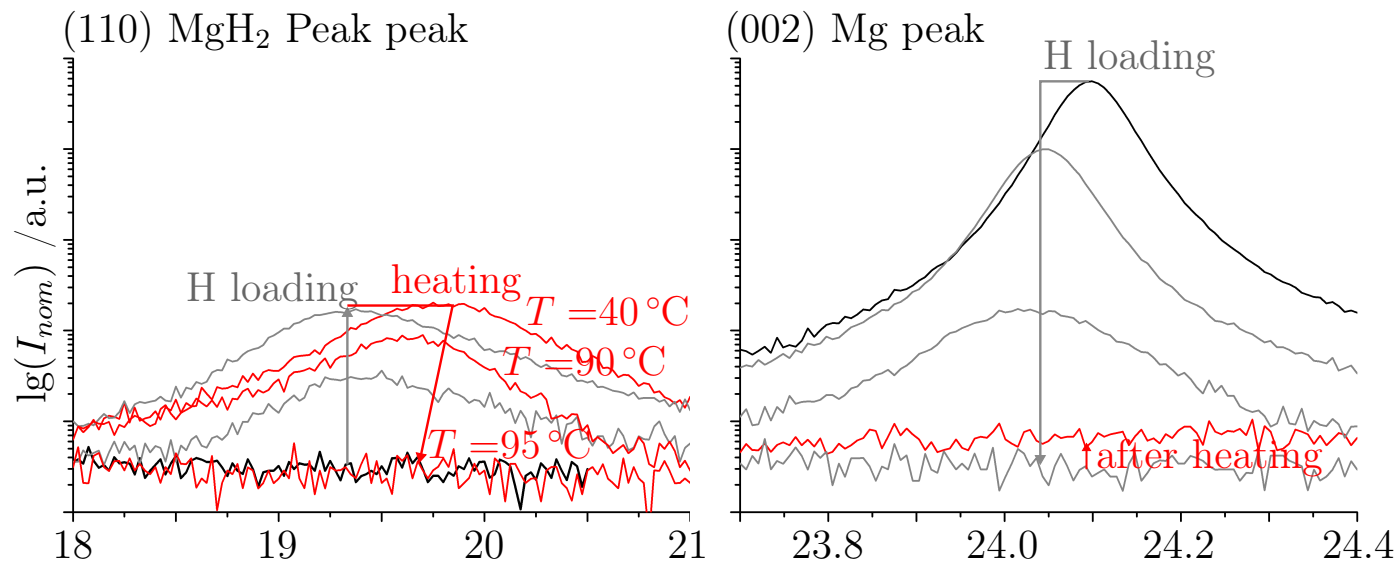

$2 \Theta$

Figure 4.10.: XRD diffractogram of (002) magnesium and (110) magnesiumhydride peak measured in situ at the ERSF in Grenoble $(\lambda=1.078 \AA)$. After starting the hydrogen loading the magnesium peak vanishes and the magnesiumdihydride forms (condition before hydrogen is marked in black). After the film is fully hydrided the hydrogen is removed and the film heated to $100{ }^{\circ} \mathrm{C}$, leading to the vanishing of the hydride peak. The curves measured without hydrogen during heating are shown in red.

\subsection{Film morphology after hydrogen loading}

Samples of all batches were studied after the hydrogen loading experiments using TEM. In addition, some were studied by XRD. The results are collected in the following. Figure 4.11 shows the grain structure of the IBS and PLD samples after they were loaded and unloaded with hydrogen (compare figure 4.2 for the structure before hydrogen loading). Again, it contains TEM bright field and dark field images of the samples and a schematic drawing of the grain structure. It can be seen that the structure of the two samples becomes more similar after hydrogen loading. 

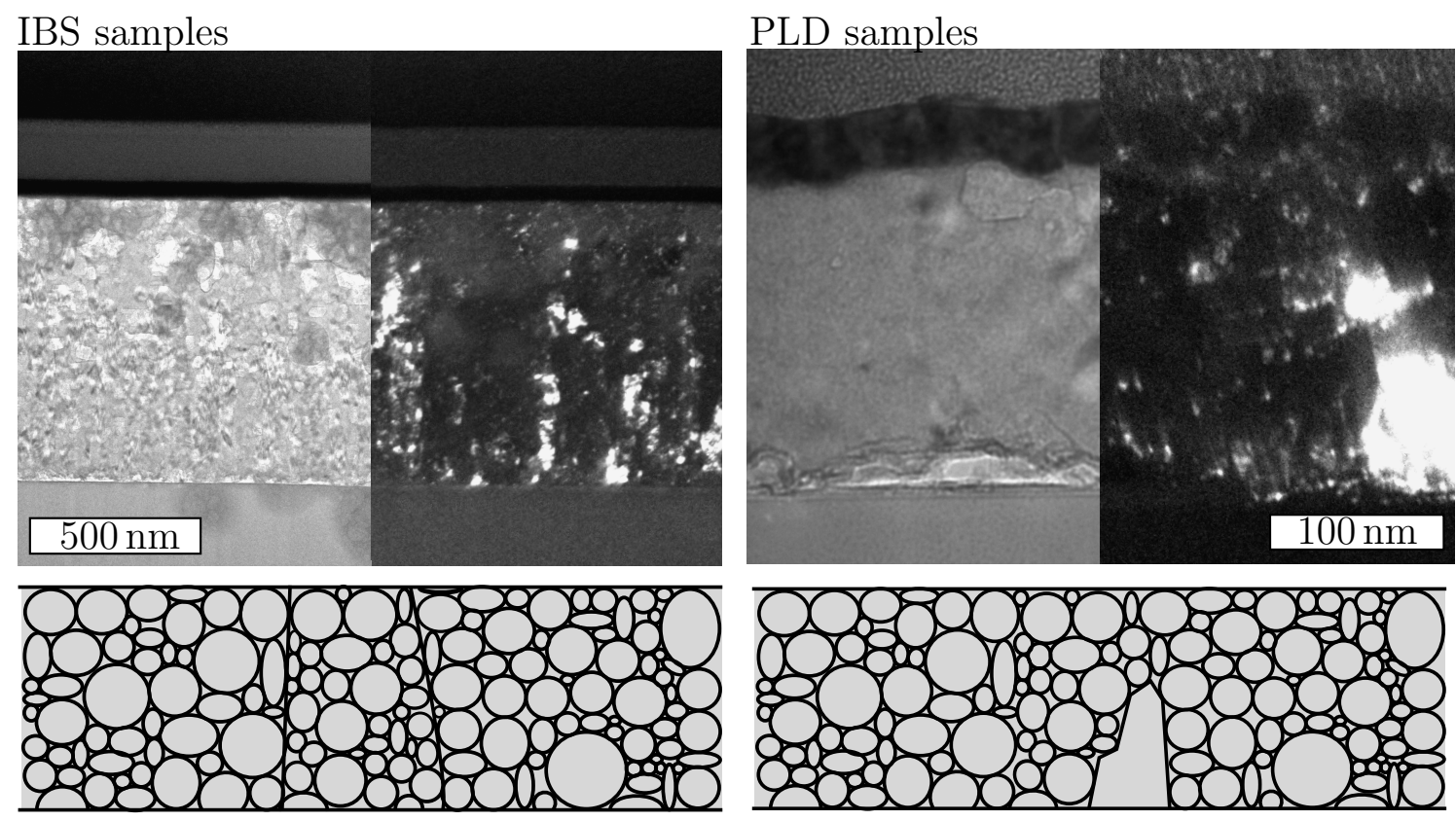

Figure 4.11.: Example of the grain morphology of the IBS samples (left) and the PLD samples (right) after hydrogen loading and unloading. For each a bright field and dark field TEM image are shown. In addition, a schematic drawing of the typical grain morphology is shown below the TEM images.

Both samples show nanocrystalline equiaxed grains, similar to those found in the in situ experiments. In the IBS samples some indication of the original columnar grains can be found. In accordance to this, XRD measurements still found (002) magnesium peaks for the samples. This indicates that some orientation remains, while the weaker peak confirms the smaller grain size. The same orientations with weaker peaks are found in the PLD samples. For these, the main difference in the grain structure is the size reduction of the large grains found in the as-prepared samples. Large grains remain, but they are smaller than before. Table 4.2 shows the results of the grain size measurements. The IBS samples show much smaller grain sizes than before and the results of the TEM and XRD measurements now fit well together. For the PLD samples only the size of the small grains is given for the TEM value. The size does decrease slightly compared with unloaded films, but the change is within the error range. The XRD value is slightly larger because it contains an influence of the remaining large grains. However, it is also decreased in comparison to the values before hydrogen loading.

The film thicknesses of the magnesium and palladium thin films were measured on samples of the same batch. One sample was measured in the initial state and one samples was measured after it was loaded with hydrogen and unloaded in air. The relative change in film thickness between the as-prepared samples of a batch and the loaded samples is given in figure 4.12. The full results can be found in table B.1 
in appendix B.1.1. While the samples of one batch were prepared at the same time, their original thickness must not be equal, which should be kept in mind if comparing the samples. Nevertheless, a general trend can be found that samples loaded with hydrogen show larger $\mathrm{Mg}$ film thicknesses. This is not true for the Pd capping layer. Only batch E shows a thicker Pd layer after loading with hydrogen and the difference falls within the error range ${ }^{2}$. A value larger than zero means the film is thicker after hydrogen loading. Almost all batches show an increase of the film thickness, only the change of batch IBS-A is slightly below zero. The black dashed line shows the theoretical linear elastic expansion calculated in chapter 2.1.2. It should be noted that this value relates to the hydrided state, not the unloaded state measured in these experiments. It therefore only gives the maximum, possible expansion for the films. The red line is the average of all batches. One can see that the average is smaller than the linear elastic theory would predict, but larger than zero. This indicates plasticity that forms during the film expansion and is not reversible. Therefore, an expansion remains after hydrogen is removed from the samples and the hydride is decomposed. While all IBS samples are below the theoretical linear elastic value the one PLD batch measured shows a large relative thickness change larger than one. It is very probable that the $\mathrm{Mg}$ film of the sample loaded was already much thicker than the sample not loaded, leading to an artificially large relative thickness increase. Another possible explanation for the large increase would be grain sliding, as nanocrystallization is almost not possible for the PLD films. Only the few, large grains (compare figure 4.2) should be able to form dislocations to create smaller grains. The small, equiaxed grains cannot form dislocation lines long enough to reduce the strain energy of the volume expansion during hydride formation (for additional information see e.g. [222] and [223]).

Figure 4.12: Comparison of the relative film thickness change after hydrogen loading for different samples. The red line is the average change of all samples. The black dashed line is the theoretical linear elastic expansion as calculated in section 2.1 .2 .

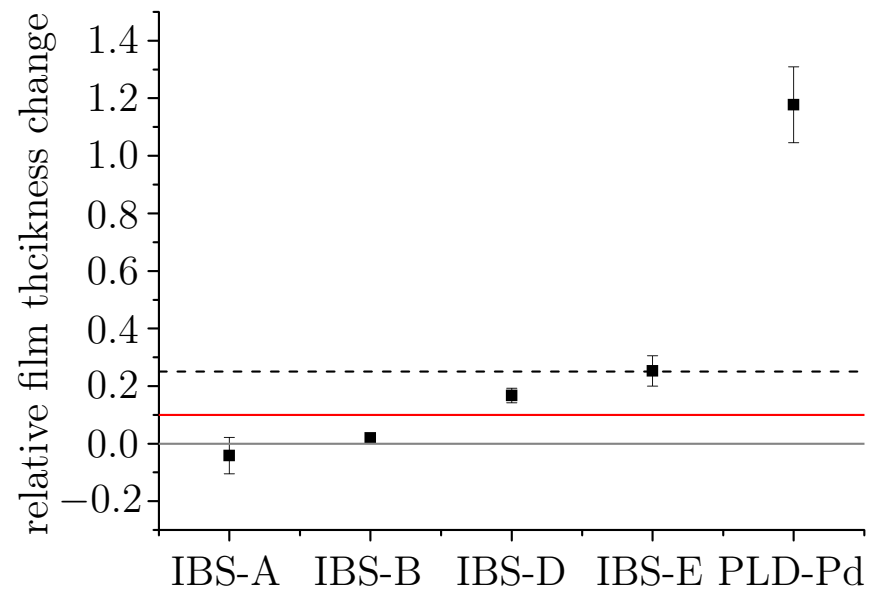

${ }^{2}$ See table B.1 in appendix B.1.1 for the full results of the palladium layer thickness. 


\section{Measurement results and individual discussion of diffusion coefficients of hydrogen in nanocrystalline $\mathrm{MgH}_{2}$}

This chapter presents the results of the diffusion coefficient measurements. In the beginning the limiting kinetic process will be evaluated. As discussed in chapter 2.1 .4 and 2.3 the hydride formation requires different steps. The slowest of these steps hinders the overall process. As in this work the diffusion coefficient is measured by evaluating the overall kinetics of the system, it is necessary that the kinetics is limited by diffusion. If this is not the case a measurement will not give a valid diffusion coefficient. After the measurements, which are limited by diffusion, are identified, the related diffusion coefficients are given in the second part of the chapter. The measured coefficients are compared with different experimental parameters to evaluate what influences the diffusion coefficient. Finally, the last part discusses the finite-element simulation results. The FEM simulation results allow to evaluate the influence of the grain and grain boundary diffusion coefficients $\left(D_{V}\right.$ and $\left.D_{G B}\right)$ on the overall system diffusion coefficient $D_{\text {sys }}$. This is essential to the discussion of the measured diffusion coefficients. The FEM results have been published in "FEM simulation supported evaluation of a hydrogen grain boundary diffusion coefficient in $\mathrm{MgH}_{2} "$ [221].

\subsection{Evaluation of the limiting kinetic process}

In the following, the results of the resistance measurements, evaluating the limiting process, will be given. Table 5.1 gives the result for all samples where a resistance measurement was accessible 1 . For each sample a JMAK plot was prepared and

\footnotetext{
${ }^{1}$ This was the case for all samples except the batch IBS-C and sample IBS-A5.
} 


\begin{tabular}{l|ll|ccccc}
\hline Sample & $\begin{array}{l}t_{\text {start }} \\
/(\mathrm{s})\end{array}$ & $\begin{array}{l}\text { percent } \\
\text { of } t_{90}\end{array}$ & $n$ & $d_{C V}$ & $R_{C V}^{2}$ & $d_{\text {Jander }}$ & $R_{\text {Jander }}^{2}$ \\
\hline \hline IBS-A1 & 55 & 86 & 0.67 & 3.5 & 0.9804 & 1.2 & 0.9998 \\
IBS-A2 & 325 & 77 & 0.82 & 1.6 & 0.9979 & 0.9 & 0.9997 \\
IBS-A3 & 500 & 71 & 0.78 & 0.5 & 0.8758 & 1.3 & 0.9971 \\
IBS-B1 & 80 & 90 & 0.46 & 3.5 & 0.9537 & 0.99 & 0.9891 \\
IBS-B2 & 0 & 100 & 0.46 & 3.5 & 0.5347 & 1.0 & 0.6249 \\
IBS-D1 & 0 & 88 & $0.77(0.92)$ & 3.5 & 0.9788 & 1.5 & 0.9943 \\
IBS-D1 & 0 & 100 & $0.75(1.1)$ & 3.5 & 0.8346 & 1.1 & 0.9772 \\
IBS-E1 3 & 0 & 40 & $0.4(1.1)$ & 3.5 & 0.7723 & 0.3 & 0.7844 \\
& 18000 & 60 & $1.3(1.1)$ & 3.5 & 0.9889 & 2.3 & 0.9892 \\
IBS-E14 & 20 & 0.7 & $1.3(0.4)$ & 1.3 & 0.9786 & 0.2 & 0.9678 \\
PLD-noPd & 16 & 96 & 0.64 & 3.5 & 0.9323 & 3.5 & 0.9945 \\
PLD-noPd & 2800 & 98 & $0.36(0.66)$ & 3.5 & 0.9624 & 3.5 & 0.9674 \\
PLD-Pd & 4620 & 71 & 1.3 & 3.5 & 0.9624 & 0.9 & 0.9940 \\
PLD-Pd5 & 250 & 69 & 1.3 & 0.9 & 0.9920 & 0.6 & 0.9900 \\
\hline
\end{tabular}

Table 5.1.: Overview over results for the limiting kinetic process of each hydride formation evaluated by resistance measurements. The first column gives the sample hydrided. $t_{\text {start }}$ is the starting time of the period in the JMAK plot with the longest constant slope. The percentage of this period compared to the total time $t_{90}$ is also given. Afterwards, the following parameters are given for the studied period: the JMAK exponent $n$ including the exponent $n_{i}$ of equation 2.30 where relevant (see also table B.2), the fitted $d$ values for the contracting volume model (CV) and Jander's model (see section 3.3.3). $R^{2}$ is the coefficient of determination for the two models. Black font indicates that the limiting process seems to be diffusion controlled, while blue fonts indicate interface/surface limited processes. For the measurement marked in red the limiting process is unclear.

the longest, straight slope in this plot was evaluated ${ }^{2}$ (compare chapter 2.3 for the theoretical background and chapter 3.2 .3 for the experimental procedure). Table 5.1 gives the starting point $t_{\text {start }}$ of the region and what percentage of the overall measurement time $t_{90}$ it takes up. The total time is defined as the time until $90 \%$ of the overall hydrided volume is reached. For almost all measurements the discussed time period contains over $70 \%$ of the entire measuring time. Only for the second loading of sample IBS-E1 a shorter time period was evaluated. It makes up only $0.7 \%$ of the measuring time. The reason for this is that the diffusion coefficient measurements were finished after this period. As the limiting kinetic process is evaluated to determine if a diffusion measurement is valid it does not make sense to evaluate time periods after the diffusion measurement. For the first loading of sample IBS-E1 two time periods are evaluated as they are similarly large and the diffusion measurements cover both.

\footnotetext{
${ }^{2}$ The overall number of regions with different slope can be found in table B.2 in appendix B.2.1.
} 
For each evaluated time period the following values are given: The JMAK exponent $n$ and the fitted dimension for the contracting volume model $d_{C V}$, as well as for Jander's model $d_{\text {Jander }}$. A value of 3.5 is set as maximum of the fit of $d$, as values above 3 have no physical meaning. For both models the coefficient of determination $R^{2}$ of the fit is given. This allows comparing the fit quality of the two models to the data, indicating which model describes the data better. Where the parameter of impingement was found to be low (see table B.2 in appendix B.2.1) the exponent $n_{i}$ of equation 2.30 is also given in brackets. It can be comparable to the JMAK exponent and may be more appropriate in these cases. Some additional values are given in appendix B.2.1.

The majority of measurements evaluated (10 of 13) can be interpreted as diffusion limited. In the following, some examples of these will be given before the three measurements with different limiting processes will be discussed.

\subsection{1. (1D) diffusion limited measurements}

Figure 5.1 shows the volume fraction of the magnesiumhydride $\phi_{M g H 2}$ plotted over time and the square root of time for sample IBS-A1. The plot given as function of time shows a line to guide the eye, which is equal to the CV model in one dimension. The same is shown in the other plot for Jander's model. The time period given in table 5.1 is marked by a red box. It can be seen that Jander's model fits the data better. The CV model in one dimension gives values below the data while a fit of the model gives the dimension of $d_{C V}=3.5$ (see table 5.1). Jander's model in one dimension gives values only slightly below the data. Because of this, a dimension of $d_{\text {Jander }} 1.2$ is given by the fit. This fits well to the JMAK exponent of $n=0.67$, which indicates a diffusion limited process with a dimension of slightly above one. Almost all samples show a similar behavior as IBS-A1. Only sample IBS-E1 and the two PLD samples differ and will be described below.

For the majority of samples the behavior can be summarized as follows (compare table 5.1). The CV model in one dimension falls below the data and most of the time the fit reaches the maximum value of 3.5. Where the maximum is not reached Jander's model still has a higher coefficient of determination $R^{2}$. Jander's model has a dimension of around one, often slightly higher. In accordance with this, the JMAK exponent is around 0.5, most of the time between 0.5 to 1 . For sample

\footnotetext{
${ }^{3}$ The JMAK plot has two regions with relevant time length. The results for both are therefore given.

${ }^{4}$ Contradictory to the other measurements, the minority part of the resistance measurement was studied for this sample as discussed in the text.

${ }^{5}$ The sample was not unloaded between the two steps. During the first step it did not load completely, a further pressure increase was needed.
} 


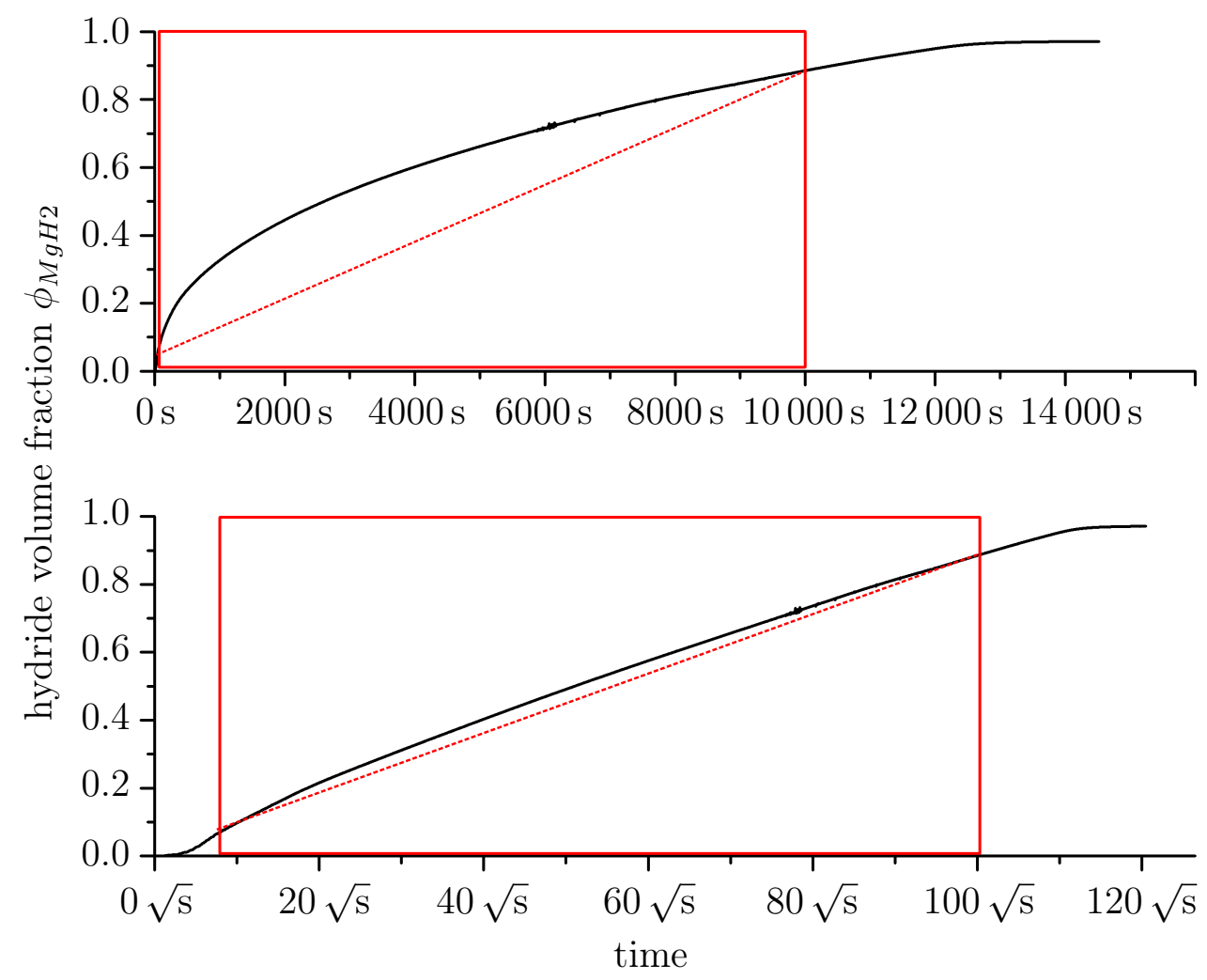

Figure 5.1.: Comparison of the volume fraction $\phi_{M g H 2}$, evaluated by resistance measurement, to the CV model (top) and Jander's model (bottom) for sample IBS-A1. The sample shows a linear plot of volume fraction over the square root of time (see the red lines guiding the eye). This indicates a kinetic limitation by a one-dimensional diffusion. The red box marks the area which was evaluated for table 5.1

IBS-D1 the impingement parameter was low. Therefore, the JMAK results have to be treated with caution. However, in this case the results of the CV and Jander's model support the JMAK result.

Figure 5.2 shows the results for the first loading of sample IBS-E1. Here, two regions are evaluated, as stated above. The first region shows the same behavior as discussed above for IBS-A1 and other samples. The fitted dimension of the Jander's model $d_{\text {Jander }}$ is very low with 0.3 (see table 5.1). However, the CV model shows an worse fit and the JMAK and impingement fits also indicate a probable diffusion limitation with a dimension of around one. The second region in figure 5.2 shows a different behavior. Again, the fit of Jander's model is better than of the CV model, but Jander's model gives a dimension of $d_{\text {Jander }}=2.3$. This fits to the JMAK exponent of $n=1.3$, which can indicate a diffusion limitation with a dimension between 2 to 3 A fit to the Ginstling-Brounshtein model (see chapter 2.3) in two and three dimensions represent the data better than Jander's model (see see figure B.4 in appendix B.2.1). As Jander's model does not describe diffusion processes with a 


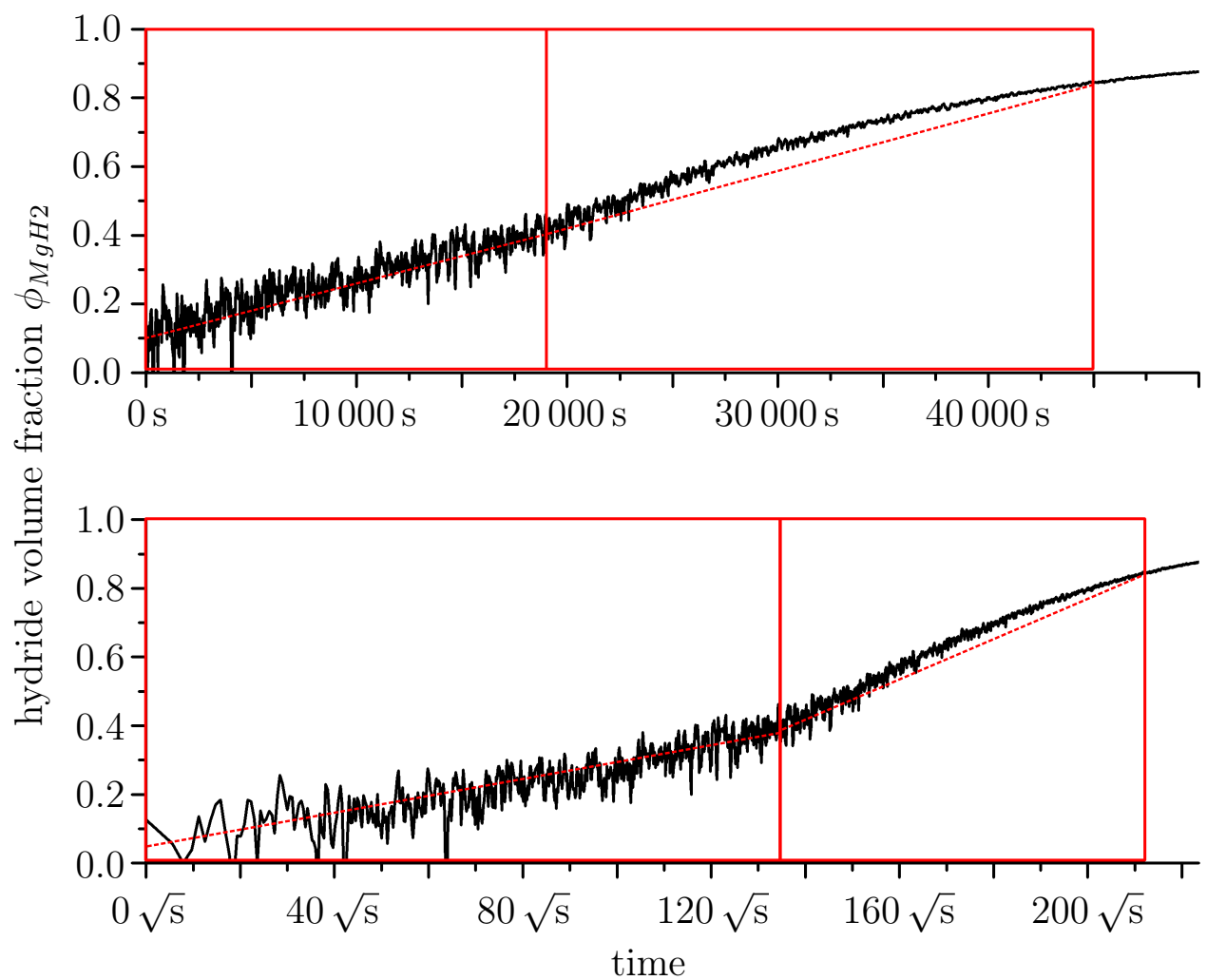

Figure 5.2.: Comparison of the volume fraction $\phi_{M g H 2}$, evaluated by resistance measurement, to the CV model (top) and Jander's model (bottom) for sample IBS-E1 (first time loaded). The sample is limited by the hydrogen diffusion, however the dimension of the diffusion process seems to change (see text).

dimension over one well, this is to be expected. Overall, it seems that sample IBSE1 is diffusion limited during the first time it was loaded with hydrogen. However, the dimension of the diffusion process changes after $18000 \mathrm{~s}$.

\subsubsection{Measurements with different limiting processes}

Overall, three samples, IBS-E1 and the two PLD samples, show behavior that indicates a non diffusion limited kinetics in some measurements. The volume fraction $\phi_{M g H 2}$ of the second loading of sample IBS-E1 is shown in figure 5.3 . The CV model in one dimension fits the data well (figure 5.3, top), while Jander's model in one dimension overestimates the data (figure 5.3 , bottom). This corresponds to a very low fit value of the dimension for Jander's model of $d_{\text {Jander }}=0.2$ (compare table 5.1). The fit of the CV model has a dimension of $d_{C V}=1.3$ and the higher coefficient of determination $R^{2}$. This indicates a limitation by an interphase or surface process. For one dimension both processes result in the same analytic description, 


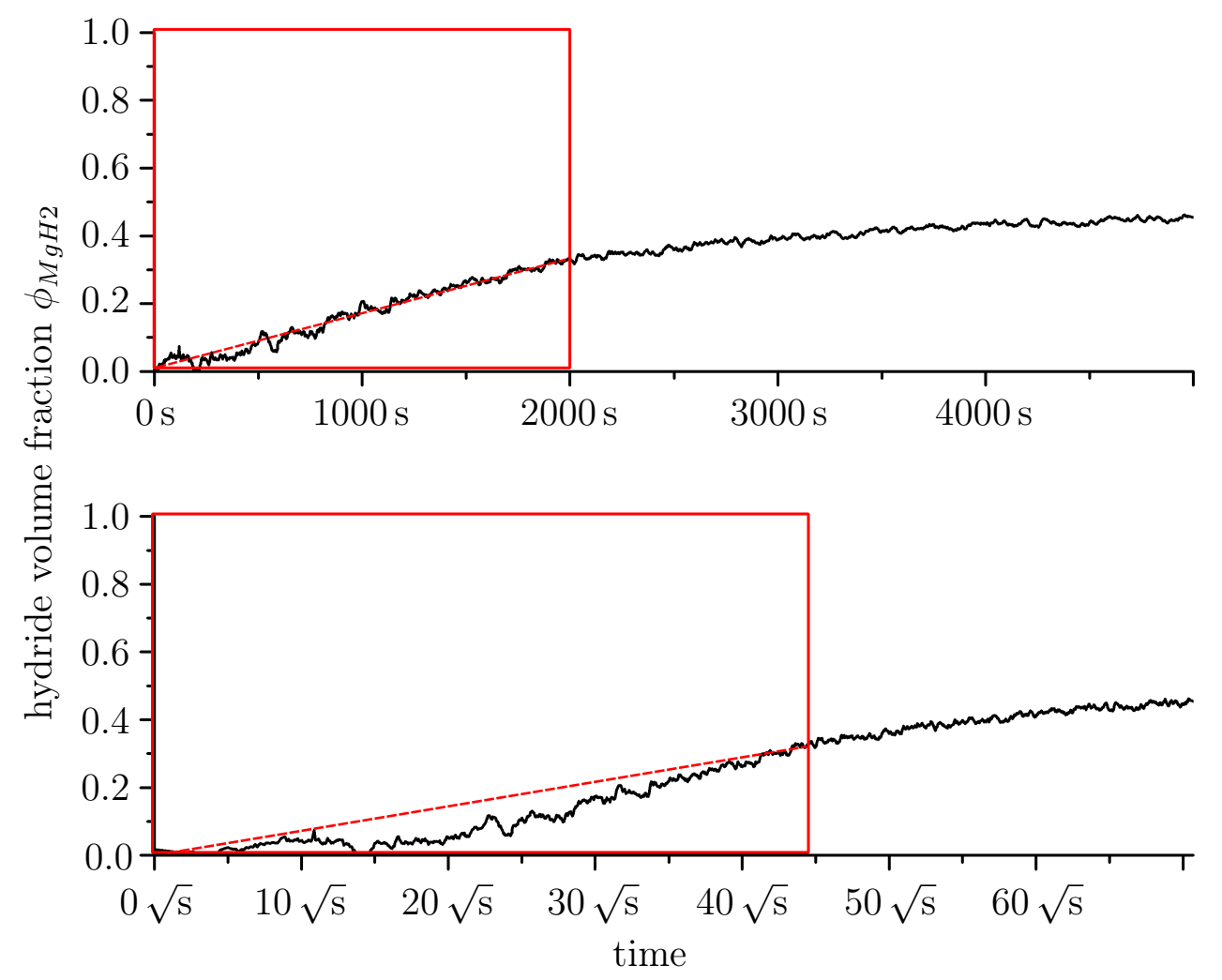

Figure 5.3.: Comparison of the volume fraction $\phi_{M g H 2}$, evaluated by resistance measurement, to the CV model (top) and Jander's model (bottom) for sample IBS-E1 (second time loaded). The sample shows a linear plot of volume fraction over time. This can be interpreted as either a limitation by a reaction interphase growth or an surface process (for both in one dimension). The red box marks the area which was evaluated for table 5.1

as discussed in chapter 2.3. The JMAK exponent is $n=1.3$, the same value as for the second part of the first loading. There, it was interpreted as a diffusion limited process. However, values between one and two are not unambiguous. Especially if the nucleation rate changes, many cases are possible. $n=1.3$ can be interphase limited for instantaneous nucleation with a dimension between one and two and decreasing nucleation (see chapter 2.3p. This would fit to the results of the CV model. The same behavior as the second loading of sample IBS-E1 was found for a second loading step of sample PLD-Pd. It was not a second loading like for IBS-E1, because the sample was not unloaded, but the hydrogen pressure was directly increased from $10 \mathrm{hPa}$ to $30 \mathrm{hPa}$. In the following, the two interphase limited measurements (marked by blue font in table 5.1) will not be evaluated for a diffusion coefficient.

Figure 5.4 shows the development of the $\mathrm{MgH}_{2}$ volume fraction $\phi_{\mathrm{MgH} 2}$ of sample PLD-Pd (second time loaded). The interpretation of the limiting process of this measurement is unclear. Neither the dimension of the CV model nor of Jander's 


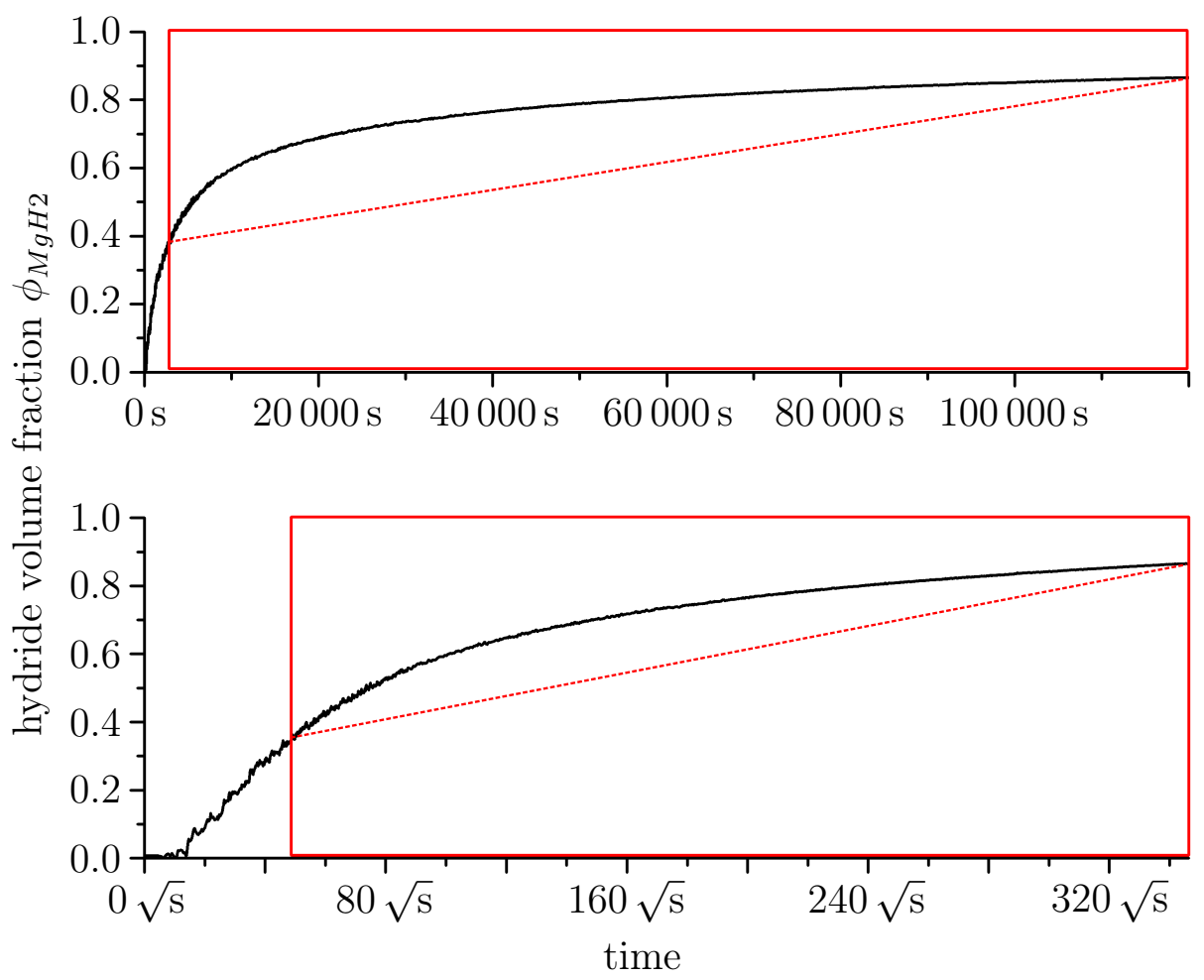

Figure 5.4.: Comparison of the volume fraction $\phi_{\mathrm{MgH} 2}$, evaluated by resistance measurement, to the CV model (top) and Jander's model (bottom) for sample PLD-Pd (second time loaded). The limiting process of this sample is unclear. The JMAK exponent is below 0.5 , while neither the CV model nor Jander's model converge for a dimension below $d<3.5$.

model converges resulting in the maximum of 3.5. The JMAK coefficient is also below the theoretical minimum of $n=0.5$. As the impingement parameter is low, this may be a result of the fact that the JMAK equation is not applicable (see table B.2 in appendix B.2.1). Together with the high dimension found in the other fits, no limiting process becomes evident. Because of this the measurement is omitted for the evaluation of a diffusion coefficient. The first loading of the sample showed the same high dimensions for the fits of the CV and Jander's model (compare table 5.1. . However, the impingement factor of the first loading is large and the JMAK exponent is at $n=0.64$. This value of $n$ is similar to the other samples, which were interpreted as diffusion limited in one dimension. The diffusion coefficient of the first loading is therefore included in the following chapter.

As stated above, for the samples of Batch IBS-C and sample IBS-A5 no resistance measurement data is available, therefore no limiting kinetic process can be evaluated for these measurements. As most measurements were found to be limited by diffusion, this is also assumed for the samples IBS-C and IBS-A5. However, it should be kept in mind that this is an assumption. 


\subsection{Results of overall system diffusion coefficient measurements}

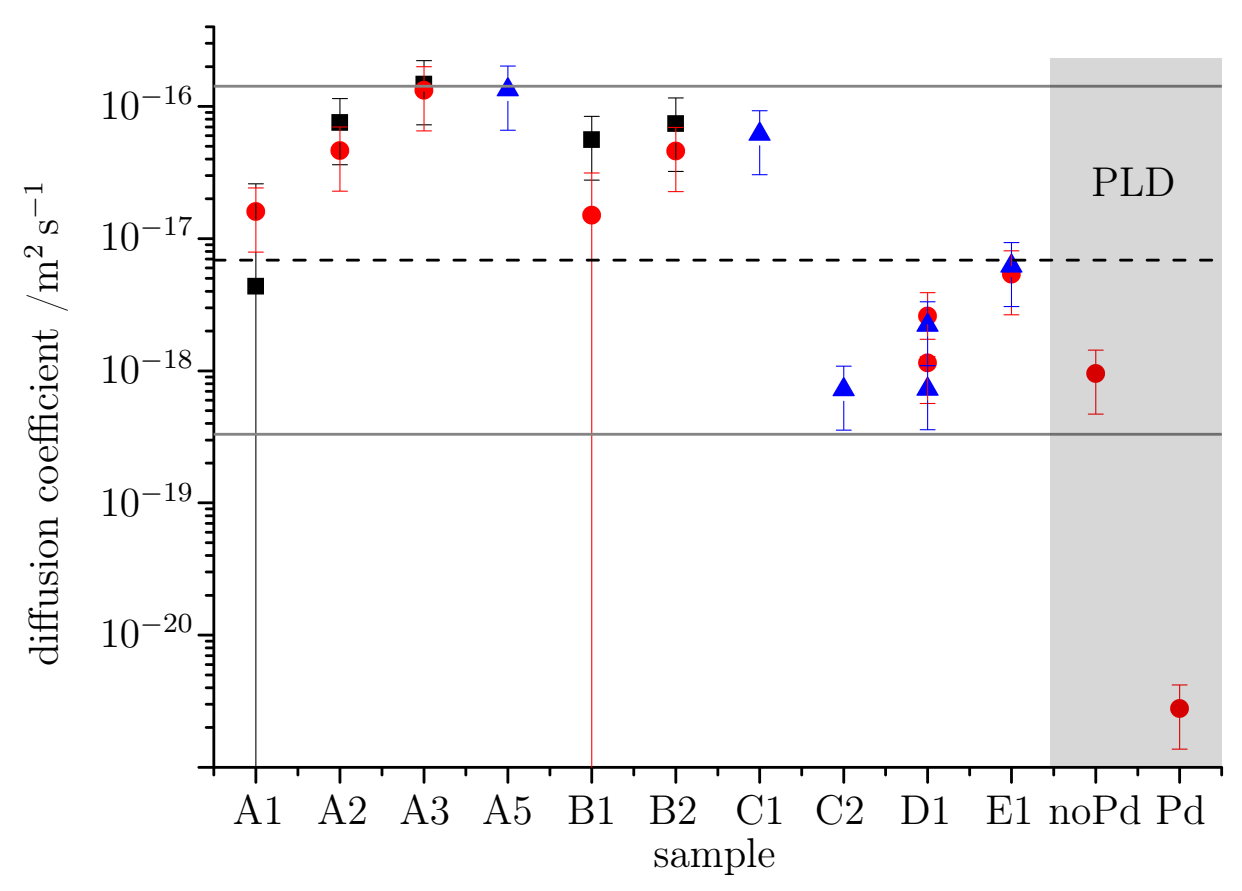

Figure 5.5.: Diffusion coefficients measured in this work sorted after sample. The PLD samples are marked by a grey box. Black squares are measured by in situ XRD measurements, blue triangles are measured by gas volumetry measurements and red circles are measured by resistance measurements.

Three methods were used to measure diffusion coefficients: X-ray diffraction (see chapter 3.5 and equation 3.20, gas volumetry (see chapter 3.2 and equation 3.10 ) and resistance measurement (see chapter 3.3 and equation 3.17). These methods give the overall system diffusion coefficient of the sample $D_{\text {ove }}^{X R D / G / R}$ (the superscript gives the measurement method). This is most likely a combination of the grain diffusion coefficient $D_{V}$ and the grain boundary diffusion coefficient $D_{G B}$, but the single components cannot be distinguished by the methods utilized in this work. Table B.3 in the appendix B.2.2 gives the results for the different samples measured. The same information is shown in figure 5.5. Diffusion coefficients measured by in situ XRD are plotted in black, values measured by a resistance measurement are plotted in red and the gas volumetry measurements are plotted in blue. The PLD samples are marked by a gray background. The black dashed line gives the average diffusion coefficient of all diffusion coefficients measured: $D_{\text {ove }}=6.9_{-6.5}^{+140} \cdot 10^{-18} \mathrm{~m}^{2} \mathrm{~s}^{-1}$. The gray solid lines mark are the variance. The average was calculated from the logarithm of the 
diffusion coefficients, because diffusion coefficients are expected to be log-normal distributed. Figure 5.5 shows that the measured diffusion coefficients for a single sample fit together well, independent of the method. This is even more valid if only a single loading step is taken into account 6 . Because of this, in the following the average diffusion coefficient of each loading step is discussed.
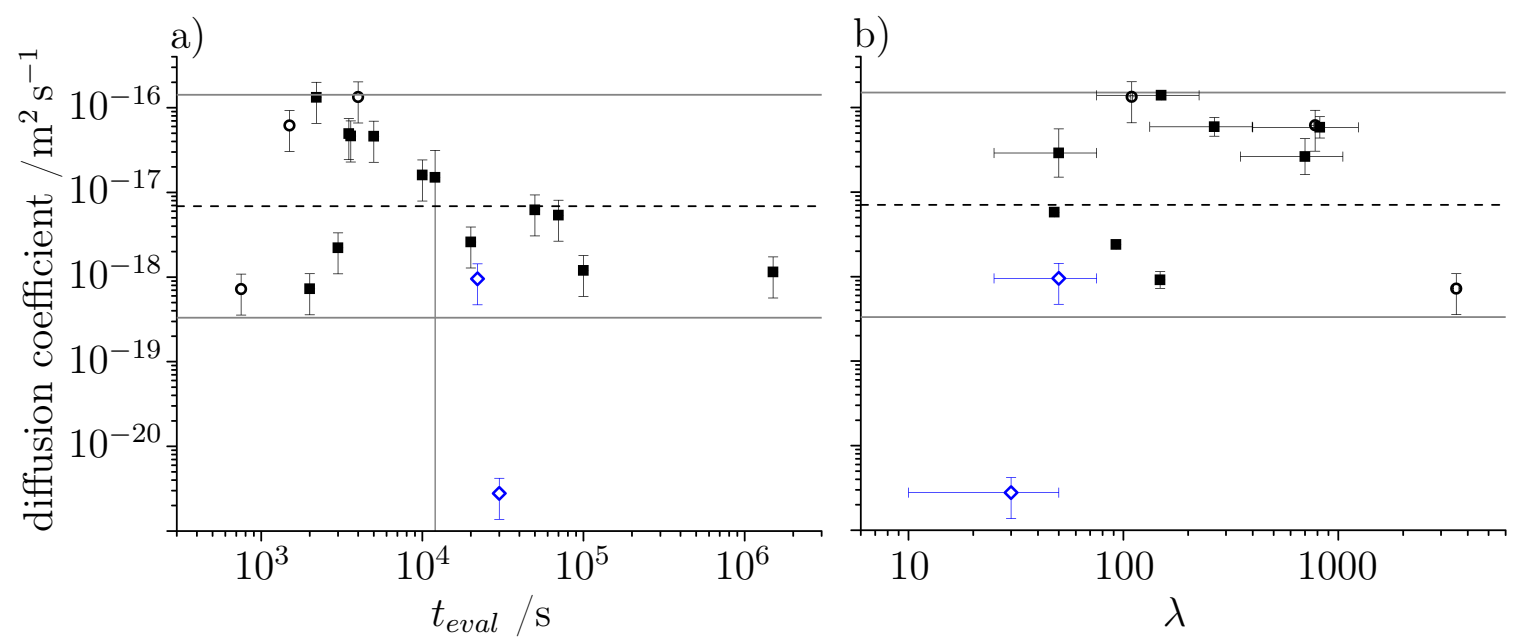

Figure 5.6.: Comparison of the diffusion coefficient with (a) the time range of the evaluation $t_{\text {eval }}$ and (b) $\lambda$. The PLD samples are marked as blue diamonds. Samples where no resistance measurement was available to measure the diffusion length are marked as open circles. The average and variance of all diffusion coefficients is marked by a dashed black line and gray solid lines respectively.

Figure 5.6 a) compares the diffusion coefficients evaluated by gas volumetry and resistance measurements with the evaluated time $t_{\text {eval }}$. Both methods are based on the diffusion from a well-stirred fluid of limited volume using the first term approximation as discussed in chapter 3.2.3 and 3.3.3 (equation 3.10 and 3.17). The first term is an appropriate approximation for long measuring times. Therefore, for short times a change in the measured diffusion coefficients can occur as the approximation becomes invalid. This is not found for the measurements in this work.

Figure 5.6 b) compares the diffusion coefficients with $\lambda$ (compare equation 3.5). Again, no trend is found. As discussed in chapter 3.2.2, the first term approximation is appropriate for $\lambda>0.4$. This is fulfilled for all samples. Therefore, no dependency of the diffusion coefficient on $\lambda$ is expected. In both parts of figure 5.6 the blue diamonds mark the PLD samples. While one shows a very low diffusion coefficient the other falls within the variance of all samples.

Open circles mark samples that were measured without a resistance measurement (this includes both samples of batch IBS-C and sample IBS-A5). This means that

\footnotetext{
${ }^{6}$ Only for sample IBS-D1 and IBS-E1 more than one loading step were measured.
} 
no diffusion length was measured at the same time. To evaluate the diffusion length for these samples the hydrogen concentration $c_{H}$ in the sample was calculated by equation 3.2. Assuming a maximum concentration of $c_{H}=2$ and a linear hydride front growing through the magnesium film, the volume fraction $\phi_{M g H 2}^{c}$ of the $\mathrm{MgH}_{2}$ can be estimated:

$$
\phi_{M g H 2}^{c} \approx c_{H} / 2
$$

For some samples this value can be compared with the volume fraction $\phi_{M g H 2}^{R}$ as calculated from the resistance values (see figure B.5 a) in appendix B.2.2). It was found that $\phi_{M g H 2}$, as calculated via the concentration $c_{H}$ is smaller than when calculated via the resistance $R$. This is important to remember as the resistance already underestimates the hydride volume fraction $\phi_{M g H 2}$ (see chapter 3.3.2).

Figure 5.7: Comparison of the diffusion coefficient with the grain size. The PLD samples are marked as blue diamonds. Samples where no resistance measurement was available to measure the diffusion length are marked as open circles. The average and variance of all diffusion coefficients is marked by a dashed black line and gray solid lines respectively.

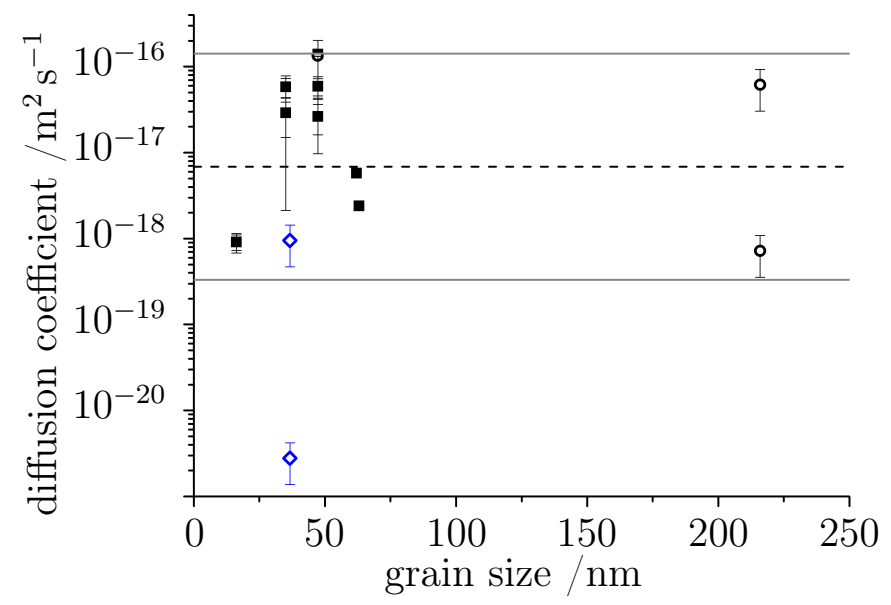

As discussed in chapter 2.2.4 grain size can influence the diffusion coefficient in polycrystals because it changes the amount of influence the volume and grain boundary diffusion coefficients have on the system diffusion. Therefore, figure 5.7 presents the diffusion coefficient as a function of the grain size of the sample. The in-plane grain size of the as-prepared samples was applied for anytime a sample was measured for the first time (see table 4.2 and chapter 4.1). If the sample was loaded a second time the grain size of the loaded and unloaded sample was applied (see table 4.2 and chapter 4.3p. This size was measured after all loading experiments were finished. Therefore, it is implied that the grain structure changes during the first loading but not significantly afterwards. This is assumed because of the results shown in chapter 4. No dependence on the grain size can be found. The PLD samples may be, on average, slower than the IBS samples but this is not a clear dependence.

Two possible dependencies of the diffusion coefficient on experimental parameters were found in this work. The first is shown in figure 5.8. The diffusion coefficients are shown as function of the hydrogen loading pressure $p_{H}$. A possible increase for 
increasing hydrogen pressure is found and indicated by a red dotted line. It will be discussed in chapter 6.3 . Figure 5.9 shows the other possible dependence. The diffusion coefficient is plotted as function of the iron content of the samples. A linear fit shows the increase of $D$ with increasing iron content. The diffusion coefficients are plotted linearly to clarify the linear dependence. As before, the black dashed line still shows the average diffusion coefficient and the gray solid lines the variance. 


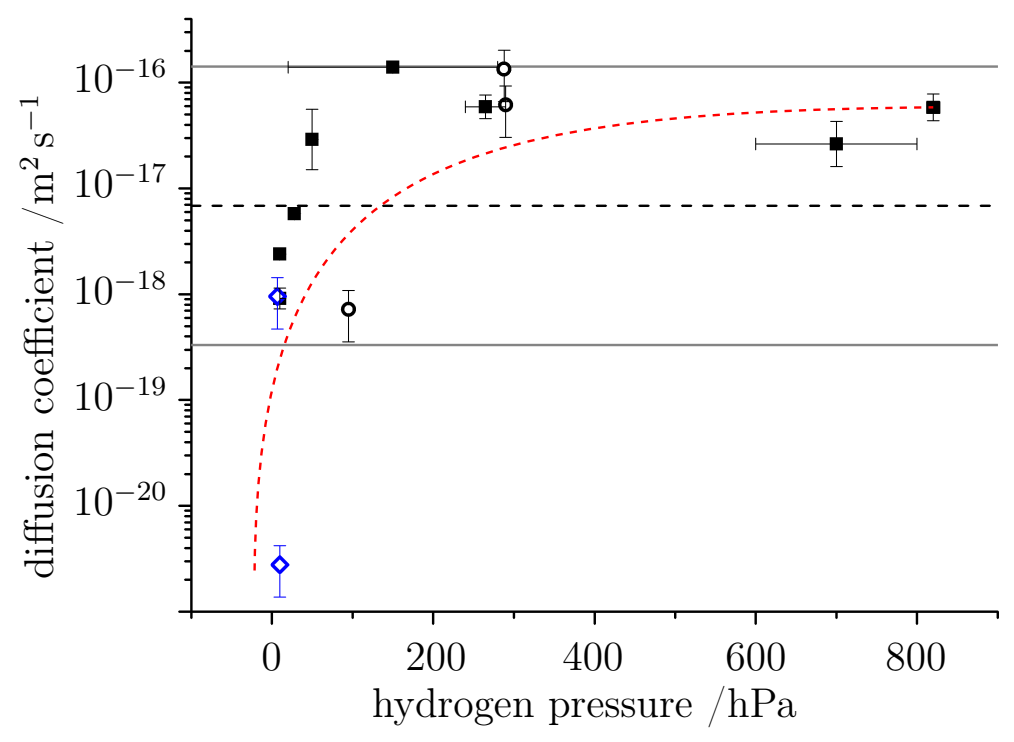

Figure 5.8.: Comparison of the diffusion coefficient with the hydrogen loading pressure $p_{H}$. The PLD samples are marked as blue diamonds. Samples where no resistance measurement was available to measure the diffusion length are marked as open circles. The average and variance of all diffusion coefficients is marked by a dashed black line and grey solid lines respectively. The red dashed line is a guide-of-eye for a possible correlation between $p_{H}$ and $D$.



Figure 5.9.: Comparison of the diffusion coefficient $D$ with the iron content of the sample. The PLD samples are marked as blue diamonds. Samples where no resistance measurement was available to measure the diffusion length are marked as open circles. The average and variance of all diffusion coefficients is marked by a dashed black line and gray solid lines respectively. The red fitting function shows the linear dependence between $D$ and the iron content. 


\subsection{Results of the FEM simulations}

To determine how the grain boundary diffusion $D_{G B}$ coefficient influences these overall diffusion coefficients $D_{\text {ove }}$ FEM simulations were performed (see chapter 3.6). The results of the FEM simulations will be presented in this section. For each simulation the single diffusion coefficients of the grain $D_{V}$ and the grain boundary $D_{G B}$ are known. Therefore, changes in the overall diffusion coefficient can be correlated with the single components. First, it will be evaluated how well each of the Harrison regimes describes the data sets at different grain sizes and ratios of $D_{V} / D_{G B}$. Therefore, a simulation time of $t=10 \mathrm{~s}$ will be evaluated. Afterwards, the influence of time will be shown by comparing the results at $t=10 \mathrm{~s}$ with results at $t=100 \mathrm{~s}$.

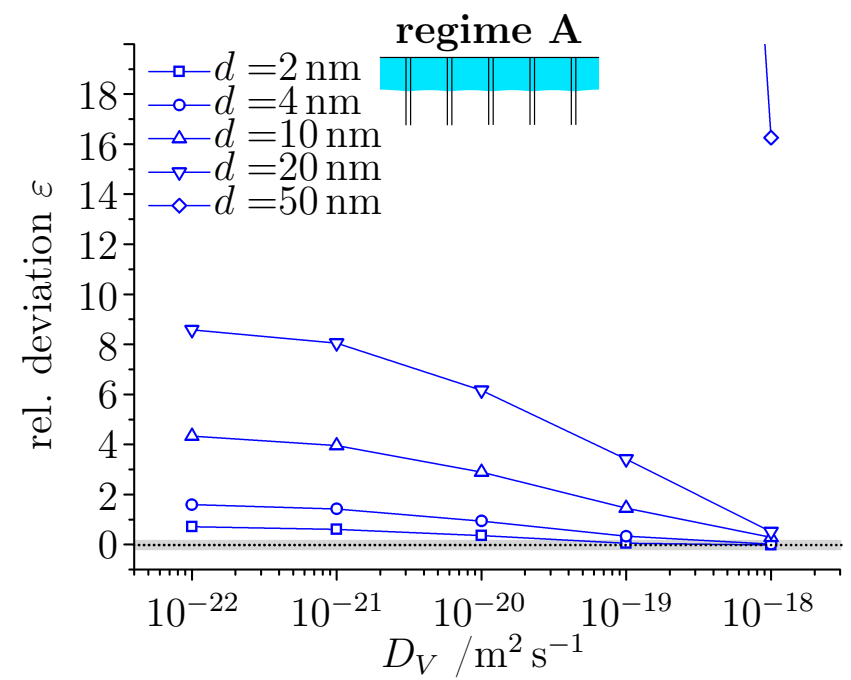

Figure 5.10: Relative deviation of the extracted grain boundary diffusion coefficient $D_{G B}^{e x t}$ as function of the grain diffusion coefficient $D_{V}$. The data was evaluated at the simulation time of $t=10 \mathrm{~s}$. $D_{G B}^{e x t} \quad$ was evaluated assuming regime A and equation 3.21 The gray area marks a rel. deviation -0.2 $<\varepsilon<0.2$. The figure is reproduced from M.Hamm et al. 221

Figure 5.10 shows the relative deviation $\varepsilon$ for the extracted grain boundary diffusion coefficient $D_{G B}^{e x t}$ in regime $\left.A\right]^{7}$. The dotted black line indicates $\varepsilon=0$, where the extracted grain boundary diffusion coefficient $D_{G B}^{e x t}$ is equal to the original value of $10^{-17} \mathrm{~m}^{2} \mathrm{~s}^{-1}$ put into the simulation. It can be seen that regime $\mathrm{A}$ works best for the highest $D_{V}$ and the deviation to data of the FEM simulation results gets larger with lowering $D_{V}$. Furthermore, a strong influence of the grain size can be found. The relative deviation $\varepsilon$ gets larger by increasing the grain size and the deviation between different grain sizes gets larger for lower values of $D_{V}$. For $50 \mathrm{~nm}$ large grains the simulation gives already at $D_{V}=10^{-18} \mathrm{~m}^{2} \mathrm{~s}^{-1}$ a relative deviation of $\varepsilon=$ 16. This deviation increases further by decreasing $D_{V}$ up to a value of $\varepsilon=228$. For small grain sizes of $d=2 \mathrm{~nm}$ the relative deviation only grows from $\varepsilon=-0.02$ to $\varepsilon=0.71$.

\footnotetext{
${ }^{7}$ See chapter 3.6 .1 about how the relative deviation $\varepsilon$ is calculated.
} 
Figure 5.11: Rel-

ative deviation of the extracted grain boundary diffusion coefficient $D_{G B}^{e x t}$ plotted as function of the grain diffusion coefficient $D_{V}$. The data was evaluated at the simulation time of $t=10 \mathrm{~s}$. $D_{G B}^{e x t}$ was evaluated assuming regime $\mathrm{B}$ and equation 2.21. The gray area marks a rel. deviation $-0.2<\varepsilon<0.2$. The figure is reproduced from M.Hamm et al. 221

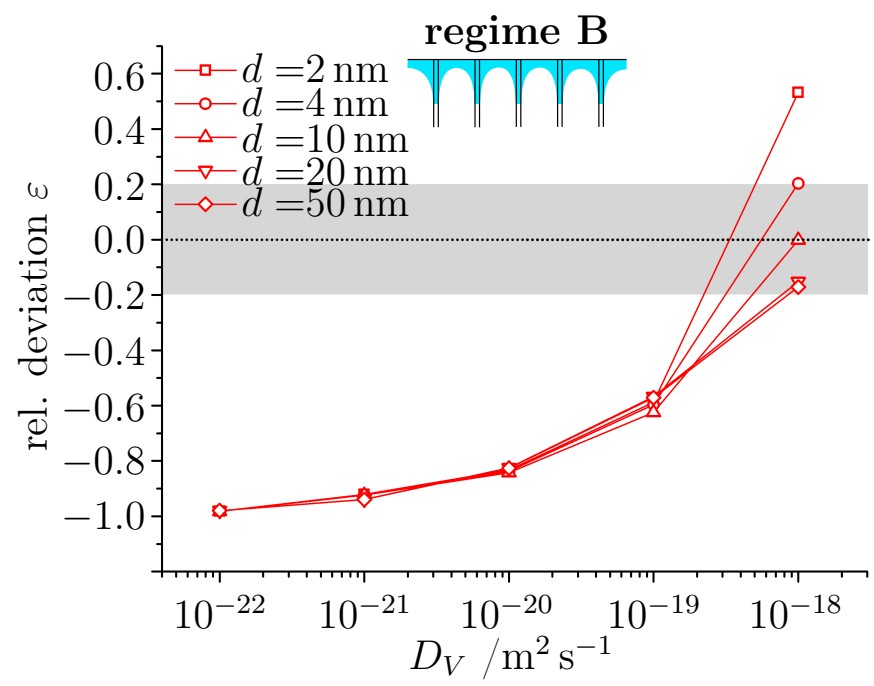

Figure 5.11 shows the relative deviation $\varepsilon$ assuming regime B. It can directly be seen, that the deviation for regime B stays smaller than with regime A (compare figure 5.10). The maximal relative deviation for all grain sizes and $D_{V}$ studied is $\varepsilon=-0.98$. Also, the deviation is negative for most parameter combinations, which means that the true grain boundary diffusion coefficient is underestimated by regime B. In regime A, the grain boundary diffusion was mainly overestimated. Thus, at the given time of $t=10 \mathrm{~s}$, regime B works best for $D_{V}=10^{-18} \mathrm{~m}^{2} \mathrm{~s}^{-1}$, with some minor dependence on the grain size. For smaller values of $D_{V}$ the relative deviation gets larger, however the dependence on the grain size does vanish with smaller $D_{V}$.

Figure 5.12 shows the relative deviation $\varepsilon$ assuming regime C. Opposite to the results assuming regime B (compare figure 5.11), the deviation gets smaller with decreasing $D_{V}$ in figure 5.12 . Regime $\mathrm{C}$ generally underestimates the true grain boundary diffusion coefficient as $\varepsilon<0$.

To study the impact of time on the diffusion regimes different loading times have been studied. Figure 5.13 compares the results for $t=10 \mathrm{~s}$ (on top) with the results at a time of $t=100 \mathrm{~s}$ (on bottom). The relative deviation $\varepsilon$ of all three Harrison regimes are summarized in one figure. Each regime is colored according to figures 5.10 , 5.11 and 5.12, meaning regime $\mathrm{A}$ is plotted in blue, regime $\mathrm{B}$ in red and regime $\mathrm{C}$ in black respectively. The comparison of the two loading times allows evaluating the time dependency on the relative deviation, for each regime itself and in comparison to the other regimes. As already discussed, at $t=10 \mathrm{~s}$ regime $\mathrm{A}$ or $\mathrm{B}$ give the best description of the set grain boundary diffusion coefficient for larger 


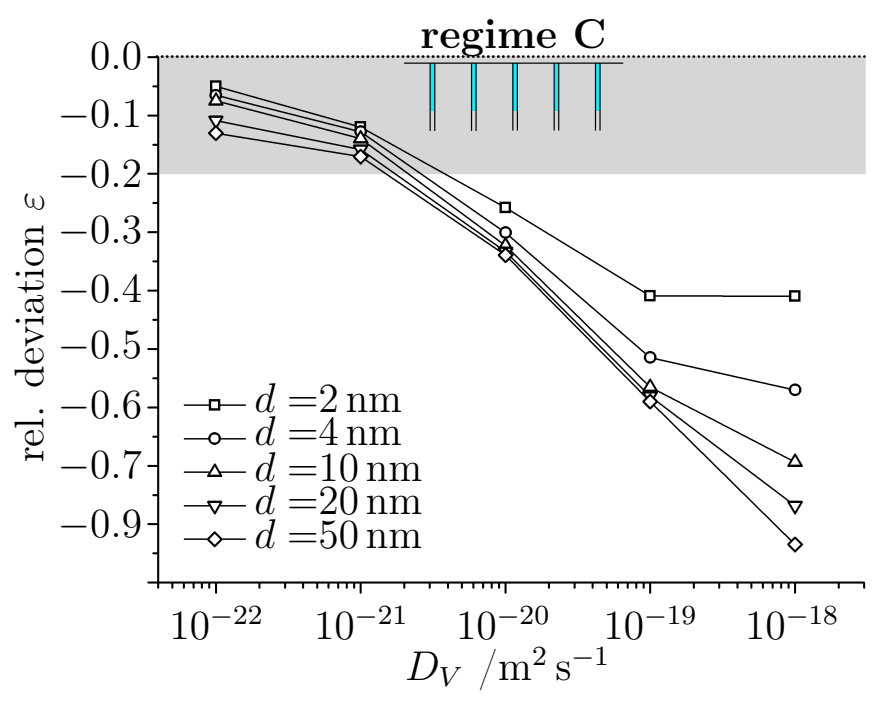

Figure 5.12: Relative deviation of the extracted grain boundary diffusion coefficient $D_{G B}^{e x t}$ plotted as function of the grain diffusion coefficient $D_{V}$. The data was evaluated at the simulation time of $t=10 \mathrm{~s} . D_{G B}^{e x t}$ was evaluated assuming regime $\mathrm{C}$ and equation 3.21. The gray area marks a rel. deviation $-0.2<\varepsilon<0.2$. The figure is reproduced from M.Hamm et al. 221

$D_{V}$. In general, it can be concluded that for very small $\mathrm{Mg}$ grain sizes regime $\mathrm{A}$ is the best assumption, while for larger $\mathrm{Mg}$ grain sizes regime $\mathrm{B}$ may give better values. However, for longer times regime A becomes the better description for increasing grain sizes. Regime $\mathrm{C}$ gives the best assumption of the grain boundary diffusion coefficient for smaller values of $D_{V}$ (this corresponds to a large value of $\Delta$ ). This result does not depend on the grain size.

Figure 5.13 shows that with increasing time and a given $D_{V}$ the systems diffusion kinetics transforms from one regime into the next. For example, while at $t=10 \mathrm{~s}$ and $D_{V}=10^{-20} \mathrm{~m}^{2} \mathrm{~s}^{-1}$ regime $\mathrm{C}$ gives the best approximation for the chosen $D_{G B}$, this changes for $t=100 \mathrm{~s}$. Here, regime $\mathrm{B}$ has a smaller relative deviation $\varepsilon$ than regime $\mathrm{C}$ and, for small grain sizes, the deviation of regime A is even smaller. But, one can still summarize that regime $\mathrm{C}$ is the best approximation for small $D_{V}$. While evaluating the simulation data, it should be kept in mind that the simulations did stop after $t=100 \mathrm{~s}$. This maximum of the time sequence was chosen because the maximal diffusion length was only $100 \mathrm{~nm}$. For longer times the analytical solution for a isotropic front is no longer valid (see equation 3.21 in chapter 3.6.1), as it assumes a semi-infinite medium. This approximation does not hold for longer times. The obtained results can be easily rescaled to longer times $t$ and larger distances $l$, using $D \approx l^{2} / t$. For example, if the sample allows for diffusion lengths in the micrometer range (10 times larger) figure 5.13 gives the appropriate regimes at times of $t=$ $1000 \mathrm{~s}$ and $t=10000 \mathrm{~s}$. Thus, the figures can be applied to many sizes of $\mathrm{Mg}$ samples, employing easy rescaling. 

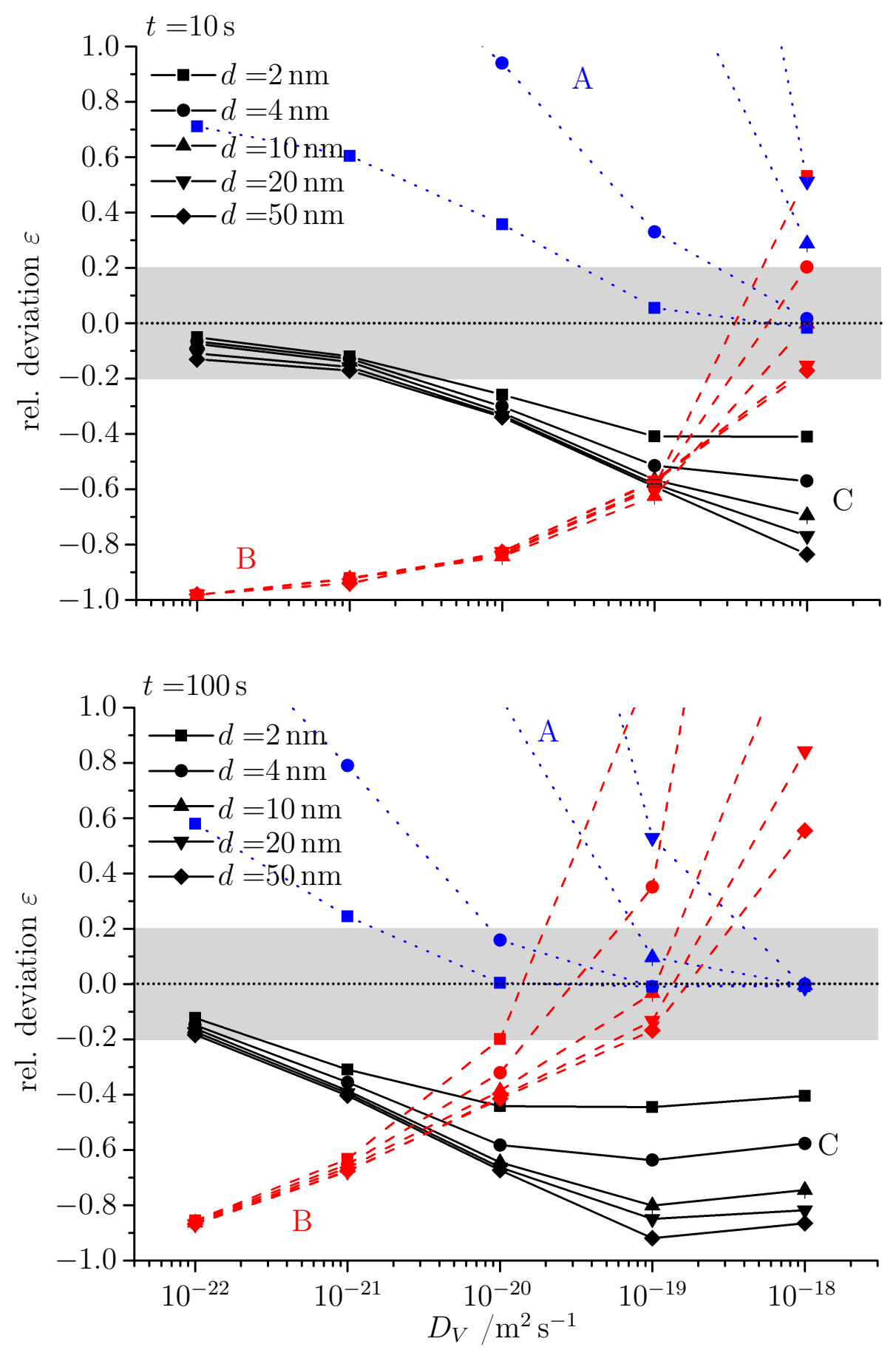

Figure 5.13.: Relative deviation of the extracted grain boundary diffusion coefficient $\varepsilon$ to the set value of $D_{G B}=10^{-17} \mathrm{~m}^{2} \mathrm{~s}^{-1}$ plotted as function of the grain diffusion coefficient $D_{V}$. The top figure was evaluated at the simulation time $t=10 \mathrm{~s}$. The bottom figure was evaluated at the simulation time $t=100 \mathrm{~s}$. The gray area marks a rel. deviation -0.2 $<\varepsilon<0.2$. Regime $\mathrm{A}$ is marked in blue and dotted lines, regime $\mathrm{B}$ in red and dashed lines and regime $\mathrm{C}$ in black and solid lines. The figure is reproduced from M.Hamm et al. 221] 


\section{Global discussion of the experimental results}

In the following, the results presented in chapter 4 and 5 will be discussed. First, the morphology of the samples and their structural changes will be compared with the literature. Thereafter, the FEM simulations will be discussed. Their comparison with literature allows for more insight into the influence of the grain boundaries on the overall diffusion process in the $\mathrm{Mg}-\mathrm{H}$ system. This knowledge is applied afterwards to discuss the experimentally evaluated overall diffusion coefficients. By taking into account the results of the FEM simulations, the presence or absence of dependencies on grain size and iron content, as well as the hydrogen loading pressure can be explained. Finally, after increasing the gas pressure surrounding an already hydrided sample a short-term increase and long-term decrease was found (compare figure 4.9). This effect will be discussed below. The chapter is concluded by taking the results of this work and showing how they may improve magnesium for a hydrogen storage application.

\subsection{Structural changes in Mg thin films by hydride formation}

The first part of this chapter compares the sample grain structure with other $\mathrm{Mg}$ thin films studied in literature. Afterwards, the changes during hydride formation are discussed with special regards to the influence of internal stresses that are created during the formation of $\mathrm{MgH}_{2}$.

\subsubsection{Film structure after preparation}

A columnar film morphology was found for the IBS samples (see chapter 4.1). The magnesium grows in an $(002) /(004)$ orientation on the (100) oriented substrate (see figure 4.1). During hydrogen loading a (110) oriented $\mathrm{MgH}_{2}$ peak appears (compare 


\section{Global discussion of the experimental results}

figure 4.10). The out-of-plane grain size after preperation was similar to the film thickness, only at the substrate interface smaller grains with out-of-plane grain sizes of about $100 \mathrm{~nm}$ to $200 \mathrm{~nm}$ were found (compare figure 4.2). The in-plane grain size is dependent on the film thickness (see figure 4.3). A similar initial film morphology was found by Uchida et al. and $\mathrm{Qu}$ et al. [44, 76, 140]. Both groups prepared $\mathrm{Mg}$ thin films by sputter deposition on (100) silicon substrates, as it was done in this work. A (002) Mg peak was found by both groups after preparation. This peak vanished during hydrogen loading and a (110) $\mathrm{MgH}_{2}$ peak appears. Uchida et al. found the same columnar grain structure in their films before hydrogen loading [76]. In addition, they found an increase of the in-plane grain size with increasing film thickness similar to what was presented in figure 4.3. Singh et al. prepared thin $\mathrm{Mg}$ films on silicon substrates by sputter deposition, as well as pulsed laser deposition [37. The results Singh et al. found for the sputter deposited thin films are similar to the films studied in this work and the films prepared by $\mathrm{Qu}$ et al. and Uchida et al. For the PLD thin films a different structure was found, as will be discussed below.

The PLD thin films studied in this work show a nanocrystalline equiaxed grain structure, containing some large columnar grains. The large grains are similar to the grain structure found in the IBS films. The nanocrystalline grains are much smaller than the grains in the IBS samples, with sizes of about $15 \mathrm{~nm}$. The PLD films show the same $(002) /(004)$ Mg orientation on (100) silicon substrates 1 . Singh et al. found a different film morphology for their PLD magnesium thin films [37. Droplets, similar to those discussed in chapter 3.1.2, are present on the samples. Singh et al. reduced the number of droplets by increasing the distance between target and substrate. However, they were not able to produce droplet free samples, as it was done in this work. The PLD films themselves showed a columnar structure similar to the sputter deposited films. However, an increased number of polycrystals and grain boundaries were described. Also, XRD studies found additional (10-10) and (10-11) peaks.

The large grains still present in the PLD films studied in this work may be comparable to the results of Singh et al. However, the small polycrystals, described by Singh, make up the majority of thin films in this work. The reason may lie in the different preparation parameters of Singh et al., indicated by the still present droplets. A comparison to the films of Singh seems to indicate that the reduction of droplets also leads to a reduction of grain size. However, this may not be a direct correlation. The droplet density in this work was reduced by reducing the laser flux on the target. This should lead to less material deposition on the substrate. Especially, it may reduce the deposition of complexes in the size of several atoms and clusters. These may act as pre-formed grain nuclei that grow further, while at other places the grain structure must nucleate and grow on its own. Hence, the grain

\footnotetext{
${ }^{1}$ Additional XRD results confirming the structure are shown in appendix $\mathrm{B} .1 .2$
} 
structure becomes more nanocrystalline as fewer large grains grow from pre-formed nuclei. Finally, the PLD films prepared in this work do not show different XRD peaks than the IBS films. Only the (002) Mg peak was detected.

\subsubsection{Influence of hydride formation: internal stresses and their consequences}

The ETEM results give a good insight into the changes the $\mathrm{Mg}$ thin films undergo during the hydride formation. As figures 4.5 and 4.6 show, the hydride forms in the thinnest part of the Mg film (Window 1, see figure 4.4). Afterwards, it grows roughly half-spherical. At the interface to the $\mathrm{Pd}$ capping layer fingers form and grow outwards through the TEM lamella.

Several things can be taken from these results. First, the hydrogen seems to enter from the palladium (and platinum) covered side. This can be concluded from the growth behavior of the hydride, which indicates that additional hydrogen only enters through the magnesium/palladium interface. This behavior can be easily explained by a magnesiumoxid layer that is known to form on magnesium (compare chapter 2.1.4). The oxide forms on the sides of the lamella, only leaving a clean interface to the substrate and the $\mathrm{Pd}$ capping layer. The absorption of hydrogen is prevented by the oxide. Therefore, hydrogen only can enter through the Pd capping layer.

Second, only one hydride nucleus forms in the lamella and it does so in the thinnest part of the lamella (see figure 4.4 for the relative thickness of the lamella). The hydride formation in $\mathrm{Mg}$ thin films has been discussed by Uchida et al. [44, 76]. It was proposed that the hydride nucleates at the palladium/magnesium interface, growing afterwards in a half-spherical shape. At some point it will form a closed layer, which grows further through the film ${ }^{2}$. The Uchida model describes many phenomena found in the ETEM lamella correctly, e.g. the initial nucleation at the palladium/magnesium interface and the half-spherical growth. However, it needs to be taken into account that the lamella is not identical to a thin film. One main difference is the stress that forms during the volume expansion upon formation of the hydride (see chapter 2.1.2 and the discussion in the following).

In accordance with the differences of a lamella to a thin film, the growth of the hydride phase was found to be slightly different from the model given by Uchida et al. [44]. The fingers growing along the magnesium/palladium interface are not predicted by Uchida (see figure 4.6 a) to c) ). They do fit to measurements about the in-plane diffusion of hydrogen in $\mathrm{Mg}$ thin films, done by Teichmann et al. [224]. A

${ }^{2}$ At this point the hydride growth slows significantly as the growth becomes limited by the diffusion through the magnesiumhydride. 


\section{Global discussion of the experimental results}

very fast in-plane diffusion was found, with a diffusion coefficient of $3 \cdot 10^{-12} \mathrm{~m}^{2} \mathrm{~s}^{-1}$. One possible explanation for this was an accelerated diffusion along the magnesium/magnesiumoxide interface in the sample. A similar effect may be present here. A diffusion coefficient for the finger has been calculated in chapter 4.2 as $2 \cdot 10^{-15} \mathrm{~m}^{2} \mathrm{~s}^{-1}$. While the growth of the finger was confirmed to be limited by diffusion, it is not clear which diffusant diffuses in which material. This makes it difficult to compare the diffusion coefficient with others. The value is much lower than the value measured by Teichmann et al., but the difference may simply be the different type of interface, which is here magnesium/palladium 3

The value calculated above is still much higher than the diffusion coefficients measured for other samples in this work. The diffusion process measured in these cases is the vertical diffusion after a closed layer was formed. The average diffusion coefficient was found to be $D=6.8_{-6.6}^{+140} \cdot 10^{-18} \mathrm{~m}^{2} \mathrm{~s}^{-1}$. A diffusion coefficient can be estimated for the same process from the in situ experiment. After the finger forms a closed layer in window 2, the hydride can be seen to grow further below it (see figure 4.6 c) and d)). By measuring the growth distance $l$ over time a diffusion coefficient can be estimated as $D \approx(1 / 2 d) \cdot\left(l^{2} / t\right)$. Thereby, $D \approx 1 \cdot 10^{-17} \mathrm{~m}^{2} \mathrm{~s}^{-1}$ was evaluated. This value is inside the variance of the average $D$ measured in the other experiments.

Finally, the preferred growth along the magnesium/palladium interface found here leads to a closed hydride layer earlier than a half-spherical growth would achieve. This is an advantage for the results in this work as the growth of a layer parallel to the surface is used as a model at different points. One being the three-layer model applied to calculate the hydride volume fraction $\phi_{M g H 2}$ (see chapter 3.3.2) and, another being the model developed to evaluate a diffusion coefficient by in situ XRD (see chapter 3.5). Therefore, if a closed layer is formed by finger growth these models can be applied with less error on the results.

\section{Stress influence on the thermodynamics}

For a thin film the in-plane expansion is almost completely prevented by the adhesion between film and substrate. As calculated in chapter 2.1.2 the volume expansion of the hydride compared to the magnesium is about $32 \%$. This large volume expansion creates large strains and stresses. Uchida et al. performed COMSOL Multiphysics simulations to show that stresses of several hundred MPa are expected to form in the magnesium and higher stresses are predicted in the growing hydride [44. Similar values were experimentally confirmed by Uchida et al. for the magnesium.

\footnotetext{
${ }^{3}$ Another explanation may lie in the change of the microstructure and the corresponding formation of high angle grain boundaries as published by Hamm et al. [210].
} 
The cross sectional TEM lamella has a different set of limiting influences, as two different in-plane directions have to be taken into account: one parallel to the electron beam direction and one orthogonal to the beam. Both are parallel to the substrate/magnesium and magnesium/palladium interfaces. The expansion in outof-plane direction can be treated as free expansion, as it is done for the thin film. However, for a thin film any direction in-plane is treated equally. For the lamella, as well as the thin film the stress in the material does depend on two components: the expansion and the limitation of the material. The overall expansion scales with the volume (see chapter 2.1.2). The limitation depends on the surface area that is prevented from expansion ${ }^{4}$. For a thin film the thickness is much smaller than the size in-plane. Therefore, the problem can be simplified by only taking into account the interface adhered to the substrate and the surface. To clarify this, one takes an exemplary thin film with in-plane dimensions $l_{1}$ and $l_{2}$ and a thickness $d$. This means the surface is equal to $2 \cdot\left(l_{1} l_{2}+l_{1} d+l_{2} d\right)$. As $d<<l_{1} \approx l_{2}$ this simplifies to $2 \cdot l_{1} l_{2}$. One surface of size $l_{1} l_{2}$ is the substrate/magnesium interface and is prevented from expansion. The other surface of the same dimension is free to expand. This means that for a given volume about half of the surface is limited from expansion, giving rise to stresses. For the lamella, still the interface between substrate and thin film prevents expansion. However, the size of this interface is not any longer much larger than the other sides, so they need to be taken into account. One take an exemplary lamella, again with a (film) thickness $d$. Let $l_{1}$ be the long side of the lamella, being orthogonal to the beam and parallel to the interface (in figure $4.4 l_{1}$ would run from left to right and $d$ from top to bottom). $l_{2}$ is the direction parallel to the beam. Now $l_{2}$ is the smallest size, but no size is as much smaller as $d$ was for the thin film. Therefore, one should take $2 \cdot\left(l_{1} l_{2}+l_{1} d+l_{2} d\right)$ as the relevant surface, but again only an area of $l_{1} l_{2}$ is limited in its expansion. This means that much less of the overall surface is limited compared to a thin film. Therefore, smaller stresses are reached in the lamella for the same volume of material expanding. Furthermore, thinner parts of the lamella have even less limited surface, as the interface area to the substrate is reduced, while the sides stay the same (in the example given thinner regions only are reduced in $l_{2}$ ).

As discussed in chapter 2.1.2 the thermodynamics of metal-hydrogen systems can change drastically under the influence of stress [84, 85, 86]. The changes include an onset of hydride formation shifted to higher hydrogen concentrations. This means that regions with lower (compressive) stresses will form hydrides before those at higher stresses. This explains why the thin films presented in this work need up to $10 \mathrm{hPa}$ to form hydrogen. A value much higher than the value of $0.3 \mathrm{~Pa}$ for bulk (see chapter 2.1.1) and even than the value of $12 \mathrm{~Pa}$ for thin films in the literature (see chapter 2.1.2). The stress present in the $\mathrm{Mg}$ thin films during loading with hydrogen (in addition to the intrinsic stress coming from lattice mismatches to the

${ }^{4}$ The influence of the free surface on the developing stresses are discussed by Burlaka et al. in the supplemental materials of citation [86]. 


\section{Global discussion of the experimental results}

substrate) increases the necessary pressure to form the magnesiumdihydride ${ }^{5}$. The difference to the literature value for thin films seems to indicate that the stresses in the thin films studied here are higher than in literature. The reason for these larger stresses is unclear. It is possible that the higher loading pressure found in this work is not only the result of changes in the thermodynamics, but also of additional kinetic barrier:6. For the TEM lamella a pressure of $6.5 \mathrm{hPa}$ was enough to form the hydride, because the lamella develops less stress upon hydride formation (see discussion above). The stress limits also the local hydride formation in the lamella. Because the stress in the thinnest part of the lamella is the lowest, this part of the lamella first forms the hydride $e^{7}$. For the thin film the stress should be much more uniformly distributed, leading to a statistical formation of hydride nuclei as discussed by Uchida et al. [44].

The large stresses described above may be the reason why the magnesiumhydride does not seem to be stable at room temperature. As discussed in chapter 2.1.1 a temperature of about $577 \mathrm{~K}$ is needed to form the magnesium phase from magnesiumdihydride. The films studied in this work transform back to magnesium for temperatures $300 \mathrm{~K}$ below this value. However, the thermodynamics influences of stress not only shift the onset of the hydride formation to higher chemical potentials 8 . At the same time the onset of the formation of the magnesium phase from the hydride phase is shifted to lower chemical potentials [84, 85, 86]. Both phenomena are a result of the reduction of the critical temperature of the two-phase region. The high (compressive) stresses therefore explain the destabilization of the hydride phase. Furthermore, air is necessary to unload the samples. The probable reason for this is that the $\mathrm{Pd}$ capping layer on top prevents an association of hydrogen atoms to a hydrogen molecule? Adding air allows the hydrogen atoms to form $\mathrm{H}_{2} \mathrm{O}$ vapor. Therefore, unloading in vacuum is limited by the desorption from the surface. For high temperatures, air seems not to be necessary as shown in figure 4.10 for the in situ XRD experiments. The high temperature of $(350 \pm 15) \mathrm{K}$ seems to allow the hydrogen to form molecules without the support of air. Opposite to the results of Paik et al., the electron beam does not seem to destabilize the hydride phase [212]. The difference may be the fact that the study in this work took place in a hydrogen atmosphere and not in vacuum. Therefore, the hydride is in thermodynamic equilibrium with the atmosphere and has no reason to form the $\mathrm{Mg}$ phase. It was not studied if the magnesium is formed under the electron beam if the hydrogen is removed. This may be of interest for future research.

\footnotetext{
${ }^{5}$ For a similar behavior in Pd1-xFex films see citation 84 .

${ }^{6}$ Other possible reasons for increases in the plateau pressure in thin films were discussed by Pivak et al. [26] and Mooij and Dam 225].

${ }^{7} \mathrm{~A}$ much more detailed discussion of the influence of local stresses on the formation of the $\mathrm{MgH}_{2}$ phase in the lamella is given by Hamm et al. in reference [210].

${ }^{8}$ The chemical potential is proportional to a fixed hydrogen concentration in the sample and a fixed hydrogen pressure surrounding the sample.

${ }^{9}$ See chapter 2.1.4 for more information about the good $\mathrm{H}_{2}$ dissociation properties of palladium.
} 
Ham et al. found formation of the $\mathrm{MgH}_{2} \gamma$-phase during the hydride formation in thin magnesium films and magnesium-niobium multilayers [71. This was not found in this work. The in situ XRD results show the formation of a hydride peak, which fits better to the theoretical value of the $\beta$-phase (see figure 4.10 in chapter 4.2. Further, no indication of the formation of the $\gamma$-phase was found in XRD measurements after the hydride formation (see appendix B.1.2). Ham et al. loaded their samples at $0.25 \mathrm{MPa}$ and $373 \mathrm{~K}$. This is the only obvious difference to the studies in this work and may explain the formation of a different phase. The higher gas pressures may support the high stresses in the thin films in the formation of the $\gamma$-phase. Nevertheless, some similar results between the work of Ham et al. and this work have been found. In both studies the respective hydride phase forms the metallic Mg phase at much lower temperatures as the bulk system. Ham et al. found hydride desorption at temperatures of $400 \mathrm{~K}$ and below in vacuum. This is similar to the desorption temperatures found in this work (see discussion above). Ham et al. argued that the destabilization is a result of the metastable $\gamma$-phase, which forms because of high stresses. In this study the destabilization was argued to directly result from the high stresses.

\section{Stress influence on the grain microstructure}

A nanocrystalline structure was found for all samples, after being hydrided. For both sample types, IBS and PLD, a nanocrystalline structure with grain sizes around $10 \mathrm{~nm}$ was found. The ETEM experiment shows that the structure already changes during the first loading and that the changes occur in the hydrided regions (see figure 4.7). The change is not reversible, as after contact with air no $\mathrm{MgH}_{2}$ can be detected anymore, but the nanocrystalline structure is still present (see figure 4.8). The change in grain structure and size explains also other results found for the thin film samples. The $\mathrm{Mg}$ (002) peak was always found to be weaker after hydride formation and decomposition. This can be explained by the smaller volume fraction of the $\mathrm{Mg}$ grains, as more relative volume is taken up by grain boundaries and other defects not contributing to the XRD peak. The same defects lead to a higher resistance of the sample after the sample was loaded and unloaded (see figure 4.9.

Overall, the nanocrystalline structure explains the experimental results in chapter 4. This structure has been published before by Hadjixenophontos et al. [226]. They showed the same structure change in $\mathrm{Mg}$ thin films loaded with hydrogen at $150{ }^{\circ} \mathrm{C}$ and $5 \cdot 10^{3} \mathrm{hPa}$. Further, they presented the same XRD peaks for $\mathrm{Mg}((002)$ peak) and $\mathrm{MgH}_{2}((110)$ peak) as in this work and in the literature [37, 44, 140]. For a film that had transformed halfway to $\mathrm{MgH}_{2}$ they found a layer of hydride on top of the unchanged Mg. This fits well to the model of Uchida as discussed in chapter 2.1.4 (compare figure 2.5). As they loaded the thin film first and prepared the TEM 


\section{Global discussion of the experimental results}

lamella afterwards it makes sense that a more homogeneous hydride layer forms compared to the in situ experiment presented above. The thin film has an isotropic stress field and therefore no preferred spots of nucleation (opposite to the case of the lamella), leading to a nucleation at several positions at once, as discussed in the Uchida model.

The question remaining is why the crystal morphology changes during hydride formation. According to the discussion above, it seems probable that the reason is the stress induced by the hydride. These stresses seem to be large enough to lead to relevant plasticity, which creates the nanocrystalline structure. The occurrence of plasticity explains also the relative change of the $\mathrm{Mg}$ film thickness presented in figure 4.12. The measurement compares a film that was not hydrided with a film that was hydrided but completely unloaded of hydrogen at the time of the measurement. Therefore the leftover expansion is not the elastic, but due to plastic deformation. This indicates plastic deformation of the $\mathrm{Mg}$ film during hydrogen loading ${ }^{10}$.

To put it in a nutshell, the study of the grain structure of the Mg thin films shows how important stresses are in thin films. For the $\mathrm{Mg}-\mathrm{H}$ system studied here on the one side they lead to drastic change in the grain morphology. On the other side, they lead to changes in the thermodynamics, increasing the necessary hydrogen pressure to form hydride, while simultaneously making it easier to decompose the $\mathrm{MgH}_{2}$ phase by decreasing the unloading pressure.

\subsection{Comparison of FEM simulations with literature}

The following two sections discuss the results of chapter 5 . However, in contrast to that chapter, the discussion will start with the results of the FEM simulations. The reason for this is that the FEM simulations allow to separate the influence of the grain boundary diffusion and the grain diffusion on the overall system diffusion process. This is helpful for the following discussion of the experimentally obtained system diffusion coefficient. The FEM simulations are compared first with the theoretical predictions of diffusion in polycrystals from the literature as collected in chapter 2.2.4. Afterwards, it is discussed what this tells us about the literature diffusion coefficients of $\mathrm{H}$ in the $\mathrm{Mg}-\mathrm{H}$ system. The literature diffusion coefficients have been presented in figure 2.6 in chapter 2.1.4.

${ }^{10}$ The creation of plasticity as a result of the high (local) stresses is discussed in more detail by Hamm et la. for the ETEM lamella in reference [210. 


\subsubsection{Comparison with theory of grain boundary diffusion}

First, the results for the three Harrison regimes will be discussed individually. Figure 5.10 shows the relative deviation $\varepsilon$ for the regime A. The deviation is always positive, meaning the extracted diffusion coefficient $D_{G B}^{e x t}$ overestimates the true value $D_{G B}$. Furthermore, the deviation gets larger for decreasing $D_{V}$ and increasing grain size $d$. All of this can be explained by the nature of $D_{\text {eff }}$ (see chapter 3.6.1), which is the measured value of experiments in regime A. As $D_{\text {eff }}$ is a volume average of the two individual diffusion coefficients, the contribution of the grain boundary diffusion coefficient gets larger with decreasing grain size. Therefore, for very small grain sizes $D_{\text {eff }} \approx D_{G B}$ and $\varepsilon$ gets small. As realistic samples commonly have larger grain sizes than described here, regime A seems to be applicable only if $D_{V} / D_{G B} \leq 10$. If this is not fulfilled, the deviation $\varepsilon$ gets larger as regime $A$ is not an appropriate description of the overall process. For small $D_{V}$ the grain boundary diffusion coefficient $D_{G B}$ has a stronger influence on the overall diffusion than regime $\mathrm{A}$ would predict. In these cases the deviation $\varepsilon$ gets larger for larger grains, as the influence of the grain is overestimated by regime $\mathrm{A}$. This leads to an overestimation of $D_{G B}^{e x t}$ to balance the overestimation of the low values of $D_{V}$.

Similar effects act in regime B, presented in figure 5.11, but have a different influence. The relative deviation $\varepsilon$ does not depend much on the grain size for $D_{V}<$ $10^{-18} \mathrm{~m}^{2} \mathrm{~s}^{-1}$, but is negative. This is a result of the vanishing influence of the grain diffusion on the total diffusion. The smaller the grain diffusion coefficients get, the smaller the transport from the GB into the grain interior is. Therefore, the average concentration at a given depth depends less on the transport through the grains but depends more strongly on the transport through the grain boundaries. This helps fulfilling the assumption of regime B that the single grain boundaries are isolated. Therefore, for a low enough grain diffusion all grain sizes give the same result. However, at the same time the leakage into the grain from the grain boundary is reduced, which makes regime B less appropriate. Because the leakage into the grain is less for low $D_{V}$ the average concentration is also lower for a given depth. This leads to a lower result of $D_{G B}$ in equation 2.21 and a negative $\varepsilon$.

Finally, figure 5.12 shows $\varepsilon$ evaluated for regime C. It is found to be negative for all $D_{V}$, getting closer to zero for low $D_{V}$. This comes up to expectations: regime C only assumes transport in the GB, which gets more valid with decreasing influence of the grain diffusion. This further explains why smaller grain sizes are better described by regime $\mathrm{C}$ behavior. For smaller grain sizes the GB has a stronger contribution to the total transport because of its larger volume ratio, thereby decreasing the deviation. Regime $\mathrm{C}$ always underestimates the true value, as any influence of the grain diffusion will decrease the overall diffusion, which is assumed to be equal to the grain boundary diffusion in regime $\mathrm{C}$. 




Figure 6.1.: Theoretical prediction of the Harrison regimes in comparison with the grain size $d$ and the grain diffusion $D_{V}$ at time $t=10 \mathrm{~s}$ and $100 \mathrm{~s}$. The grain boundary diffusion coefficient is set to $D_{G B}=10^{-17} \mathrm{~m}^{2} \mathrm{~s}^{-1}$ and the grain boundary size is assumed to be $\delta=$ $1 \mathrm{~nm}$. Black dashed lines show the grain sizes studied by FEM simulations and solid black lines give the separation from a ultrafine grained sample (lower left), to a fine grained sample (middle) and coarse grained sample (upper right).

The influence of time on the three regimes was presented in figure 5.13. It can be seen that the regime, which describes the overall process best, changes with time. This meets the expectations as e.g. regime B's requirement of isolated grain boundaries becomes less valid with increasing diffusion time. For comparison, figure 6.1 shows the theoretical predicted regime for a given grain diffusion coefficient $D_{V}$, grain size $d$ and time $t$. The grain boundary diffusion coefficient is set to $D_{G B}=10^{-17} \mathrm{~m}^{2} \mathrm{~s}^{-1}$ and the grain boundary size is assumed to be $\delta=1 \mathrm{~nm}$. Figure 6.1 shows under which condition which regime is predicted after the theory presented in chapter 2.2.4. It includes all regimes discussed in chapter 2.2.4, including regime $\mathrm{B}_{2}$, regime $\mathrm{B}_{2}{ }^{\prime}$, regime $\mathrm{B}_{4}$, regime $\mathrm{A}^{\prime}$ and regime $\mathrm{C}$ '. It should be noted again that the various apostrophed (') regimes are identical to the regimes without the apostrophe for the FEM simulations (see chapter 3.6.1). Therefore, one can concentrate on the blue marked regime $\mathrm{C}$, the red marked regime $\mathrm{B}$ and the green marked regime $\mathrm{A}$. These can directly be compared to figure 5.13 . To simplify this, the grain sizes studied in the simulation are marked by black dashed lines. The two darker shaded regions mark the transition from a ultrafine grained sample, to a fine grained sample and finally to a coarse grained sample (see figure 2.11 in chapter 2.2.4. Comparison of figure 6.1 with the results in figure 5.13 shows that the theoretical predictions fit well to many parts of the results. One example is the development of the boundary between regime $\mathrm{C}$ and regime $\mathrm{B}$. Both figures show, that for a time $t=10 \mathrm{~s}$ regime $\mathrm{C}$ is the appropriate description for $D_{V} \leq 10^{-20} \mathrm{~m}^{2} \mathrm{~s}^{-1}$. For longer times of $t=$ $100 \mathrm{~s}$, the line separating regime $\mathrm{C}$ and regime $\mathrm{B}$ in figure 6.1 shifts to the left. As discussed in chapter 5.3 , the FEM results in figure 5.13 show the same, namely that 
at $t=100 \mathrm{~s}$ no longer regime $\mathrm{C}$ but regime $\mathrm{B}$ (or for small $d$ regime $\mathrm{A}$ ) give the best description for $D_{V}=10^{-20} \mathrm{~m}^{2} \mathrm{~s}^{-1}$. The main differences between the theoretical prediction and results of the FEM simulations lie in the separation of regime B and regime A. The theory predicts for $D_{V}=10^{-19} \mathrm{~m}^{2} \mathrm{~s}^{-1}$ at $t=10 \mathrm{~s}$ that all grain sizes are described by regime $\mathrm{B}^{11}$ (compare figure 6.1). The FEM simulations on the other side show that for grain sizes of $d=2 \mathrm{~nm}$ and $d=4 \mathrm{~nm}$ regime A better describes the data. Similar results can be found for other combinations of $t$ and $D_{V}$. The FEM data of small grains is described better by regime $\mathrm{A}$ as would be expected from the theory. Finally, regime $\mathrm{B}_{4}$ should be discussed. As said in chapter 2.2.4 this regime allows no evaluation of the grain boundary diffusion as it is completely governed by the grain diffusion. In addition, it is not described by the analytical solution of regime $\mathrm{A}$ or regime $\mathrm{B} / \mathrm{B}_{2}$. For the studied parameters, the theory predicts Regime $\mathrm{B}_{4}$ to be only reached for $D_{V}=10^{-18} \mathrm{~m}^{2} \mathrm{~s}^{-1}$ at $t=100 \mathrm{~s}$. The theory would predict it for grain sizes larger than $10 \mathrm{~nm}$. However, the FEM simulations show a similar picture to other parameter combinations of the region where regime $\mathrm{B}$ meets regime A. Up to $d=20 \mathrm{~nm}$ regime $A$ describes the data well and for larger grain sizes regime $\mathrm{B}$ becomes a good description. It may be that regime $\mathrm{B}_{4}$ is not visible in the evaluation of the FEM simulations, because an contribution of the grain boundary diffusion is always assumed. Under these conditions regime $\mathrm{B}_{4}$ may look like $\mathrm{B}_{2}$ only with an additional error and increased $\varepsilon$.

\subsubsection{Comparison with literature data of $\mathrm{H}$ diffusion in the $\mathrm{Mg}-\mathrm{H}$ system}

Figure 6.2 shows the diffusion coefficients taken from literature that were already presented in figure 2.6. Some values were taken out to simplify the figure. The value measured by Toepler et al. was described by themselves as a maximum value, therefore it is left out here [147]. Stioui et al. stated that no temperature dependence was found over a range of several hundred degrees [150]. This seems unrealistic, which is why their value is not plotted. Finally, the measurement of Renner and Grabke was left out, because it was measured in $\mathrm{Mg}-2 \mathrm{wt} \% \mathrm{Ce}$ samples [143] (their result will be discussed below in section 6.3). Two new regions have been added to the plot. Like in figure 2.6 the red box marks the region of $\mathrm{H}$ diffusion in $\mathrm{Mg}$. The black box marks the region of $\mathrm{H}$ diffusion in $\mathrm{MgH}_{2}$ without any influence of grain boundaries. This includes simulations and NMR measurements. The blue box marks all diffusion processes of $\mathrm{H}$ in $\mathrm{MgH}_{2}$ that are most probably influenced by grain boundaries. This includes all experiments that measured diffusion in $\mathrm{MgH}_{2}$ and cannot separate diffusion processes in grains from processes in grain bound-

\footnotetext{
${ }^{11}$ For $d=1 \mathrm{~nm}$ it could be regime $\mathrm{A}$, however this grain size was not simulated.
} 


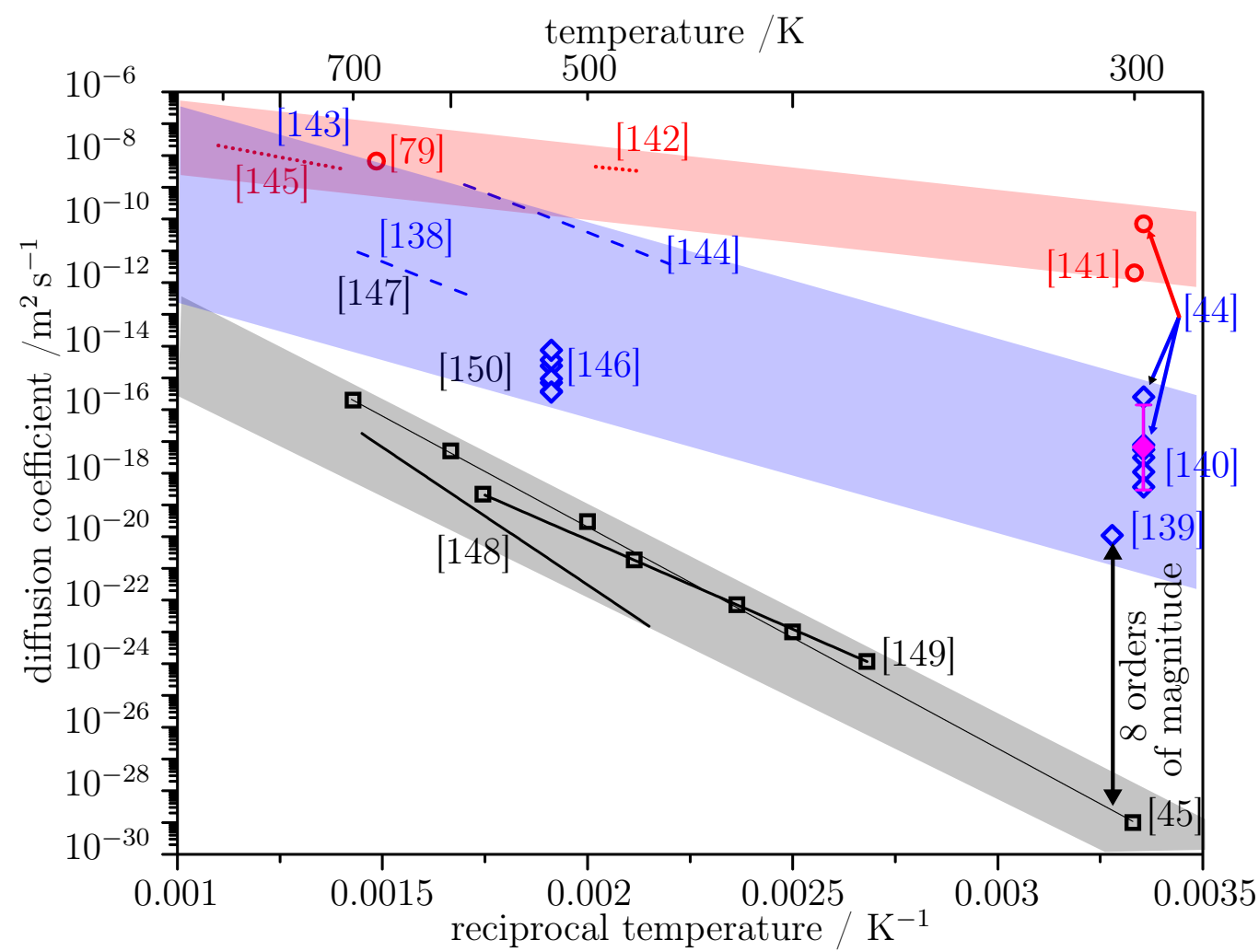

Figure 6.2.: Collection of literature data of the hydrogen diffusion coefficients in the magnesium-hydrogen system. Single diffusion coefficients, measured at a given temperature, are marked by points. Thick lines are plotted where activation energy and preexponential factor were measured. Thin lines connect measurements by a single group. The data is divided into diffusion in the $\beta$-phase (marked in black squares and solid lines), measurements during phase transformation (marked in blue diamonds and dashed lines) and in the $\alpha$-phase (marked in red circles and dotted lines). For each literature value the responding reference is given. The average and variance of all values presented in chapter 5 is given in magenta.

$\operatorname{aries}^{12}$ This region is much larger than the other two, because of a larger scatter in the literature values corresponding to this region. Generally, all regions show an increasing diffusion with increasing temperature. This is typical for diffusion processes (compare equation 2.13 and equation 2.15). Additionally, the average of all the diffusion coefficients measured in this work (see chapter 5) is given in magenta. The error is the variance of the measured values.

As indicated in figure 6.2, the difference between $\mathrm{H}$ diffusion in $\mathrm{MgH}_{2}$ with and without the influence of grain boundaries is 8 orders of magnitude, at room temperature. The large difference of values with and without grain boundaries can be explained by diffusion in Harrison regime $\mathrm{C}$. This means, the black box marks experiments

${ }^{12}$ This may be possible for simulations, experiments in single crystals or NMR experiments, which can separate different jump processes. 
measuring the grain diffusion $D_{V}$, while the blue box marks experiments measuring the grain boundary diffusion $D_{G B}$. That experiments in the blue box measure $D_{G B}$ is a result of the grain boundaries influencing the measurements (which is not the case for experiments marked by the black box). In Harrison regime $\mathrm{C}$ the diffusion process is completely governed by the grain boundaries, therefore only $D_{G B}$ can be measured (compare chapter 2.2.4). The resulting ratio $\Delta=D_{G B} / D_{V}=10^{8}$ can be compared to the results of the FEM simulations and the theory. The comparison shows that only regime $\mathrm{C}$ is expected to describe the diffusion process for this ratio. This result is independent of grain size $d$ and time $t$. At temperatures of about $T=$ $500 \mathrm{~K}$ the ratio is $\Delta \approx 10^{4}$. For even higher temperatures the diffusion time and the grain size has to be taken into account. However, as in this work all samples were measured at room temperature it can be assumed that the diffusion process can be described by Harrison regime C. Furthermore, the film thickness and times studied in the FEM simulations have to be taken into account. For diffusion over larger distances the FEM simulations can be recalculated to longer times as was described in chapter 5.3. This will be necessary for measurements in bulk systems. For diffusion lengths in the micrometer range or larger the simulations are applicable for times of 10000 seconds and more. Because the time grows quadratically it is likely that a measurement in bulk will be stopped at shorter times than studied in the FEM simulations. Hence, regime $\mathrm{C}$ becomes an even better description of the process, as for shorter times regime $\mathrm{C}$ is valid for smaller ratios of $\Delta=D_{G B} / D_{V}$ (compare figure 5.13 and 6.1. .

The fact that the data, marked in blue, directly measures $D_{G B}$ explains the larger area of the blue box. The large area is the result of a large scatter in the diffusion coefficients. As many different kind of samples have been researched (e.g. thin films vs bulk samples), very different grain boundary structures are expected, leading to significant differences in the grain boundary diffusion coefficient (see chapter 2.2.3). For example, the values measured in this work (magenta data point) fit well to the results of Uchida et al. and $\mathrm{Qu}$ et al. [44, 140]. This is to be expected as all these works studied $\mathrm{Mg}$ thin film on silicon substrates. The films showed the same grain structure (see chapter 6.1). Therefore, it seems reasonable the samples have similar grain boundary structures and grain boundary diffusion coefficient. However, if the thin films on silicon substrate are compared with other samples, e.g. thin films on Pd foils as prepared by Spatz [139], an other grain structure and orientation is expected. Having a different grain structure (and therefore a different grain boundary structure) leads to a different grain boundary diffusion coefficient $D_{G B}$ (compare chapter 2.2.3). The influence of the grain boundary structure is further complicated, as not only the structure itself is of importance but the diffusion direction in the grain boundary. Overall, the measurements in this work show also a large scatter, which will be discussed further below. The results discussed here may offer a rather simple explanation for the NMR results of Stioui et al.[150]. They found no temperature dependence on their diffusion coefficients. However, their values at high temperatures fit well to the diffusion coefficients in $\mathrm{MgH}_{2}$ without 


\section{Global discussion of the experimental results}

any grain boundaries (see figure 2.6). At low temperature the values fit well to the data from this work, Uchida et al. and Qu et al [44, 140]. It may be possible that their measurements did not measure the same diffusion process over the whole time. The measurements of Corey et al. may support this assumption [148]. Corey et al. talked about a second faster hopping process measured by NMR which was attributed to grain boundaries. No quantitative values were given but the process was described to be especially dominant at lower temperatures. All of this fits to the given interpretation of the diffusion data. Regarding the measurement of Stioui it may be that they missed the fact that two processes occur simultaneously. Over their temperature range they may have gone from the grain diffusion being dominant to the grain boundary diffusion being dominant.

The $\mathrm{H}$ diffusion in $\mathrm{Mg}$ will be discussed shortly as it was not studied directly in this work. The literature data falls in a small corridor and includes experiments on samples containing grain boundaries, as well as a simulated result of $D_{V}$ by Vegge [141]. The difference between the room temperature values of Vegge and of Uchida et al. is equal to $D_{\text {Uchida }} / D_{\text {Vegge }} \approx 45$. It should be noted that the red box cannot directly be compared with the FEM results and to figure 6.1 as all of these assume $D_{G B}=10^{-17} \mathrm{~m}^{2} \mathrm{~s}^{-1}$. However, the ratio $\Delta=D_{G B} / D_{V} \approx 45$ still seems too small to result in regime $\mathrm{C}$ (assuming that Vegge calculated $D_{V}$ and Uchida et al. measured $\left.D_{G B}\right)$. Whether regime $\mathrm{B}$ or $\mathrm{A}$ applies cannot be predicted. As discussed above, the diffusion time and grain size influences strongly which regime is an appropriate description. As these parameter are not known well enough or are too different for the different data points no general prediction can be made. In addition, it cannot be assumed that $D_{G B}>D_{V}$ for all samples. As e.g. Oudriss et al. showed trapping of hydrogen in grain boundaries has to be considered in general [56, 57]. No indication of this was found for the slow diffusion in $\mathrm{MgH}_{2}$. However, because of the very low values of $D_{V}$ in $\mathrm{MgH}_{2}$ (see black box in figure 6.2) it is reasonable that $D_{G B}>D_{V}$ is fulfilled. For the $\mathrm{H}$ diffusion in pure $\mathrm{Mg}$ this must not be true, especially considering trapping effects. As a result much of the theory of grain boundary diffusion given in chapter 2.2 has to be changed. How grain boundaries influence the diffusion of $\mathrm{H}$ in the $\mathrm{Mg} \alpha$-phase is therefore still an open question.

In conclusion, the FEM simulations have shown that Harrison regime $\mathrm{C}$ is the best description of hydrogen diffusion in $\mathrm{MgH}_{2}$ polycrystals. This result is of great importance. On the one side, it means that samples containing grain boundaries directly allow to measure the grain boundary diffusion coefficient $D_{G B}$. On the other side it means that the exact structure and properties of the grain boundaries in a $\mathrm{MgH}_{2}$ sample have to be taken into account to predict the hydrogen diffusion. 


\subsection{Influence of grain boundaries on $\mathrm{H}$ diffusion in $\mathrm{MgH}_{2}$}

The FEM simulations showed that the diffusion coefficient measured in this work is the grain boundary diffusion coefficient $D_{G B}$ (see above section 6.2.2). This result allows interpreting the dependencies found in chapter 5 . In the following, the missing influence of grain size $d$ and structure on the diffusion will be discussed. Afterwards, the dependence on the iron content will be discussed and also the apparent dependence on the hydrogen loading pressure. Finally, the influence of an additional loading step on the resistance of the thin film will be discussed (see figure 4.9 for an earlier example).

Before the dependencies, the quality of experimental results will be discussed. In chapter 5.1 it was tried to evaluate which measurements were limited by diffusion. Only these measurements were taken into account for the evaluation of the diffusion coefficient. Most measurements were found to be limited by one-dimensional diffusion (10 of 13 measurements evaluated). However, often the dimension was not exactly one, but often slightly above one (see table 5.1). This can be explained easily. A dimension of one is expected for an ideal front growing orthogonally to the layer. However, for a non ideally straight layer the overall growth does not occur perfectly orthogonal to the interfaces, leading to a slight influence of a second dimension.

For sample IBS-E1 a change in the dimension of the diffusion process was found. The beginning was found to be limited by a one-dimensional diffusion process as most other measurements. At later times it seems to be limited by a diffusion in two or three dimensions. This would mean that the hydride growth happens inplane of the thin film, which seems unreasonable. No explanation for the apparent change in the diffusion dimension can be given. The resulting diffusion coefficients fit well to the values for other measurements, which at least indicates that no drastic differences to the other samples are present.

As stated in chapter 5.2, the diffusion measurements of the batch IBS-C and IBSA5 did not include a resistance measurement and, therefore, no measurement of the diffusion length $L$. The diffusion length was estimated from the hydrogen concentration in the sample. This is less accurate than the calculation by the resistance measurement (compare appendix B.2.2). However, comparison with the other diffusion coefficients shows no general difference (see e.g. figure 5.5). The values fall in the variance of all values, with the average being slightly higher than for all other samples. It has to be taken into account that sample IBS-A5 has a high content of iron additives. In figure 5.9 it was shown that higher iron contents increase the diffusion coefficient. Because the diffusion length could be underestimated by the determination via the concentration, it would be expected that the diffusion coef- 


\section{Global discussion of the experimental results}

ficient is lower than for the other measurements. This is not the case. Overall, no clear difference of the values measured without resistance measurement to the other values can be identified. This may be because of low statistics, but it gives no reason to treat the measurements without resistance values different from the others.

Three measurements were found not to be limited by a diffusion process. One measurement of the sample PLD-noPD was not able to be fitted by any model. As the sample was prepared without capping layer, it may be concluded that an oxide layer prevents hydrogen uptake (a Pd layer was added as described in chapter 3.1.2). However, the measurement in discussion was the second time the sample was loaded. The first time showed a typical one-dimensional loading behavior and no apparent complications were found. It is therefore unclear why the second loading behaved unusually. The other two measurements (sample IBS-E1, second loading and sample PLD-Pd, second loading step) not included in the diffusion measurements were found to be limited by a one-dimensional surface or interface process. This can be the phase transformation, the adsorption on the palladium or the absorption through the interlayer between palladium/magnesium. For the second loading of sample IBS-E1 only a small part of the overall time, needed to hydride $90 \%$ of the sample, was studied. It may therefore be possible that no closed hydride layer was formed. Hence, a diffusion limitation would not be expected as the diffusion in $\mathrm{Mg}$ is fast (see chapter 2.1.4). The same argument cannot be true for sample PLD-Pd, which was measured for $69 \%$ of the full loading time (see table 5.1). However, the measurement differs from the other measurement presented. The first loading stopped forming hydride after about $22 \%$ of the sample was hydrided. This was not found for any other sample. The sample is thinner than the other samples (see table B.1 in appendix B.1.1). This can lead to higher stresses upon hydride formation [86, 88]. Apart from the many influences of stresses discussed in chapter 6.1, it leads to a slope of the plateau in the p-c-T diagram (see 2.2 for an example of a bulk system without the slope). Therefore, it may be possible, that the loading pressure of $10 \mathrm{hPa}$ applied to load PLD-Pd was enough to form some hydride but not to hydride the whole sample. Increasing the pressure to $25 \mathrm{hPa}$ allowed the whole sample to hydride. For the other samples this was not necessary as lower stresses developed during hydride formation, because of a larger film thickness. While this discussion explains why the hydride formation stopped, it does not explain why the second step is no longer diffusion limited. After the first step, it is assumed that a closed $\mathrm{MgH}_{2}$ layer has formed, which should limit the overall process. It is unclear why the further growth is limited not by the diffusion through the layer but instead by some interface.

Overall, the majority of the measurements were confirmed to be limited by a diffusion process, which allows evaluating a diffusion coefficient from the overall kinetics. In chapter 5.2 it was already discussed that no dependence on the diffusion coefficient $D$ on $\lambda$ and $t_{\text {eval }}$ is found, which confirms that the first term approximation of the solution for diffusion from a limited volume works well. Carman and Haul stated 
that pressure steps should be kept small, so a constant $D$ and $K$ can be assumed in a single phase (see section 3.2.2. This was not always followed here. However, a comparison with the diffusion coefficients measured by in situ XRD show no relevant difference (see figure 5.5 in chapter 5.2 and table B.3 in appendix B.2.2). These measurements did use a different model (see chapter 3.5 and equation 3.20 and therefore can validate the other results.

\subsubsection{Influence of grain size and structure}

No influence of the grain size on the diffusion coefficient was found (see figure 5.7). As the measurement was done in Harrison regime $\mathrm{C}$, this is to be expected. As discussed in chapter 2.2.4, the grain size does not influence the diffusion in regime $\mathrm{C}$, as only the grain boundary takes part in the transport process. This explains why no difference was found between the first time a sample was hydrided and the second time. As discussed in chapter 6.1 the grain structure changes drastically during the first time hydride forms. The different grain structure would be expected to change the diffusion coefficient of the overall system for any Harrison regime except regime $\mathrm{C}$. Another advantage regime $\mathrm{C}$ gives for the evaluation is the fact that in regime $\mathrm{C}$ moving grain boundaries do not change the diffusion speed (see 2.2.4 and citation [174]). This is of importance, as otherwise the change in grain structure during the measurement would influence the measurement. For regime $\mathrm{C}$ this can be assumed to be insignificant. The change in grain structure however may be more complex in its description than a simple moving grain boundary. The formation of new types of grain boundaries probably does influence the transport. Further, this process is probably different each time and for each sample, leading to a somewhat different grain boundary structure for each sample. This may be one explanation for the large scatter found between different measurements.

As discussed in section 6.1.2, the grain structure changes during hydride formation. The discussed reason for this are stresses arising during the hydride formation, which influence the thermodynamic behavior. However, up until now it was not discussed how stress may influence the diffusion. Stress changes the chemical potential $\mu$. Thereby, it changes the diffusion, as the chemical potential is the driving force of the diffusion process (see equation 2.7 in chapter 2.2.1). For a uniform stress it results in the typical description that the process is driven by a gradient in the concentration. However, in the thin films studied in this work stresses do not form uniformly. Already hydrided regions show the formation of plasticity as discussed in chapter 6.1.2. This lowers the stress in these regions, probably leading to nonuniform stress contributions. Ostrovsky discussed the influence of stress fields on the grain boundary diffusion in Harrison regime C [227]. He gives a stress dependent 
diffusion coefficient:

$$
D(\sigma)=D_{0} \exp \left(-\left(\frac{E_{A}-\frac{\sigma_{i i}}{3} \Omega}{R T}\right)\right)
$$

$D_{0}$ and $E_{A}$ are the pre-exponential factor and the activation energy as already discussed in equation 2.13 and equation 2.15 in chapter 2.2.2. $\sigma_{i i}$ is the first invariant of the stress tensor, given in Einstein convention. $\Omega$ is the diffusion activation volume. It can be seen that the stress increases or decreases the activation energy, depending on its direction. This means that the diffusion coefficient can be higher or lower depending if tensile or compressive stresses are present. Different stress states can, for example, be researched by changing the film thickness. Thin films are known to prevent the formation of dislocations [88, 228], leading to higher compressive stresses during loading with hydrogen. After equation 6.1 higher compressive stresses will lower the activation energy of the jump process, thereby increasing the diffusion coefficient. This fits to the results of Qu et al. [140. They found increasing diffusion coefficients from $3.7 \cdot 10^{-19} \mathrm{~m}^{2} \mathrm{~s}^{-1}$ to $7.8 \cdot 10^{-18} \mathrm{~m}^{2} \mathrm{~s}^{-1}$ with decreasing film thickness from $20 \mathrm{~nm}$ to $100 \mathrm{~nm}$.

\subsubsection{Influence of Fe content}

Iron was found to have an influence on the grain boundary diffusion coefficient $D_{G B}$ (see figure 5.9. However, Iron is not the only additive, but only the one with the highest concentrations. However, as described in chapter 2.1.4 the other additives (chromium and nickel) showed similar behavior to iron when added to the $\mathrm{Mg}-\mathrm{H}$ system. The influence of iron on the kinetics was discussed in chapter 2.1.4. The improvements in the kinetics of the $\mathrm{Mg}-\mathrm{Fe}-\mathrm{H}$ system are normally associated with improved hydrogen catalysis compared to the pure magnesium. However, the effect found here is an improvement in the diffusion, more exactly an improvement in the grain boundary diffusion. Iron is known to have no solubility in magnesium [69. It seems reasonable that iron therefore segregates along grain boundaries. No XRD peaks were detected that can be ascribed to iron or an iron containing phase, supporting the assumption. As discussed in the theory it is not surprising that a change of iron segregation changes the grain boundary diffusion coefficient. Two effects seem theoretically possible:

- Fe segregated along the grain boundary changes the relevant grain boundary size $s \delta$,

- Fe changes the grain boundary structure and/or energy, thereby changing the diffusion coefficient.

The first effect can be excluded in regime $\mathrm{C}$, as the size of the grain boundary does 
not influence the overall system diffusion (see figure 5.7). Therefore, only a change in the grain boundary structure and/or the grain boundary energy remains. The exact effect however was not studied in this work and remains for future research. Furthermore, it has to be confirmed that the influence does depend only on the iron content and not on the other additive content in the sample.

Interestingly, a similar result to the improved diffusion in samples containing iron has been published in the literature. As described in chapter 2.1.4. Renner and Grabke measured the diffusion coefficient in $\mathrm{Mg}-2 \mathrm{wt} \% \mathrm{Ce}$ samples [143]. They measured samples containing grain boundaries, therefore the diffusion coefficient measured is assumed to be $D_{G B}$. Their value is the highest value of $D_{G B}$ found in the literature (see figure 2.6). The reason may be similar to the one associated to the iron additives. However, opposite to the $\mathrm{Mg}-\mathrm{Fe}$ system, cerium forms alloy phases with magnesium (for a phase diagram see [229]). Therefore, the improved kinetics may be a result of the precipitates, which offer fast diffusion paths, instead of grain boundaries. Boulet and Gerard found that the $\mathrm{CeMg}_{12}$ phase in the $\mathrm{Mg}-10 \mathrm{wt} \% \mathrm{Ce}$ samples produces $\mathrm{CeH}_{3}$ particles during reaction with hydrogen [230]. Furthermore, Boulet and Gerard described "many incoherent grain boundaries", which may offer faster diffusion paths, leading to the high diffusion coefficient measured by Renner and Grabke.

Mintz et al. found an even more similar effect to the influence of iron discussed here [23, 151, 231]. They found the hydride formation in magnesium containing small amounts of $\mathrm{Al}, \mathrm{Ga}$ and In limited by diffusion. This is similar to the results found for most samples studied in this work. The activation energy of the hydride formation was lower for those samples containing additives compared to pure Mg. While it cannot be said with absolute certainty that the reduces activation energy is the grain boundary diffusion activation energy, it seems highly likely that this is the case. The hydride formation activation energy should be dominated by the diffusion activation energy for a diffusion limited process. Furthermore, Mintz et al. do state that the diffusion is accelerated by the lowering of the activation energy. Finally, the results presented above show that no diffusion in the grains is expected and the grain boundary dominate the diffusion. All of this would lead to the conclusion that the additives lower the grain boundary diffusion activation energy and thereby the activation energy of the hydride formation. A possible reason for this lowering of the grain boundary diffusion activation energy may be a change in the grain boundary structure, induced by additives.

A second apparent dependence on the grain boundary diffusion coefficient was found. With increasing loading pressure an increasing diffusion coefficient was measured. However, the samples loaded at high pressures were also the ones containing more iron. Therefore, in figure 6.3 diffusion coefficient are plotted as function of the loading pressure after the iron content of the samples was corrected. For the correction of the iron content the red linear fit shown in figure 5.9 is subtracted from the diffusion 


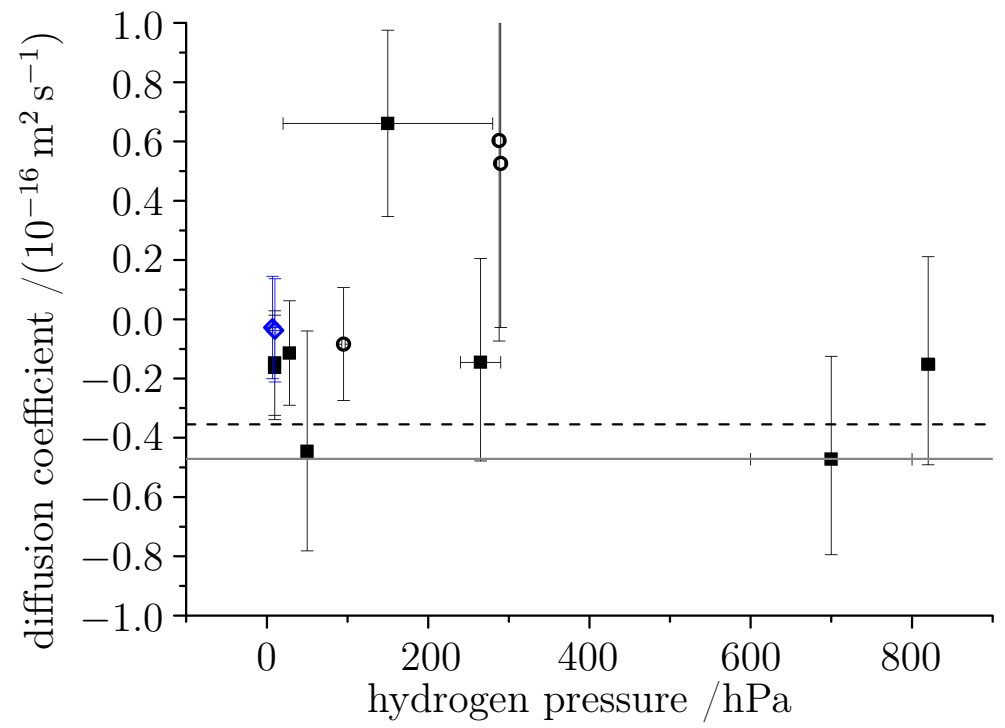

Figure 6.3.: Comparison of the diffusion coefficients, corrected for the influence of iron, with the hydrogen loading pressure $p_{H}$. The PLD samples are marked as blue diamonds. Samples where no resistance measurement was available to measure the diffusion length are marked as open circles. The average and variance of all diffusion coefficients is marked by a dashed black line and gray solid line respectively.

coefficients. While this leads to unrealistic (partially negative) diffusion coefficients, it gives an idea about the influence of the pressure excluding the influence of the iron content. As can be seen in figure 6.3, the corrected diffusion coefficients are no longer dependent on the pressure. This shows that one has to be careful when evaluating which parameter changes the diffusion coefficient as the sheer number makes the analysis difficult.

\subsubsection{Influence of changes in $\mathrm{H}$ concentration in grain boundaries in $\mathrm{MgH}_{2}$}

During two measurements a sample has been fully hydrided and afterwards the hydrogen pressure was increased further. One of these measurements was already shown in figure 4.9 in chapter 4.2. The other example is given in figure 6.4. For both the sample was hydrided at a pressure of $7 \mathrm{hPa}$ to $10 \mathrm{hPa}$ before increasing the pressure further to $100 \mathrm{hPa}$. The pressure increase leads to an increase in the resistance on a time scale of about $1000 \mathrm{~s}$. As noted in chapter 4.2 this increase is much less significant when the hydride volume fraction $\phi_{M g H 2}$ is plotted over time. In addition to the resistance increase on shorter time scales, a resistance decrease on 
much longer time scales is visible in both figures. The decrease has been measured for up to $8 \cdot 10^{6} \mathrm{~s}$, but it still did not reach an equilibrium state. While it seems in figure 6.4 that the resistance decrease follows the resistance increase, a decrease was already visible after the original hydride formation in figure 4.9. In figure 6.4 the hydride formation is not completely finished before the $\mathrm{H}$ pressure is increased, which may be why the decrease is not visible before. Because the pressure increase does not seem to be necessary for the resistance decrease it will be discussed separately from the increase.



Figure 6.4: Black curve: development of resistance of a magnesium thin film during hydrogen loading (for a second time). Blue curve: volume fraction of the magnesiumhydride calculated from the resistance measurement. The red boxes mark a loading pressure of $7 \mathrm{hPa}$. During the remaining time a higher pressure of $100 \mathrm{hPa}$ was applied.

Figure 6.4 shows not only a resistance increase upon increasing the hydrogen pressure but also a resistance decrease as the pressure was shortly reduced back to $7 \mathrm{hPa}$. The resistance does not fall back to the original value. However, the equilibrium value of the resistance had not been reached yet, which is why the resistance decreases to a higher value than it started from after the pressure increase. Increasing the pressure again brings the resistance back up. Overall, it seems that increasing the pressure surrounding a hydrided sample only creates an offset to higher resistance values, while not changing the behavior of the sample. The explanation for this lies in the fact that magnesiumdihydride is a stoichiometric phase and therefore does not take up additional hydrogen (see the bulk phase diagram, figure 2.1 in chapter 2.1.1). However, the same is not true for grain boundaries. Additional hydrogen taken up by grain boundaries explains the increase in resistance found. As discussed in chapter 2.1.3. Giebels et al. found a much lower specific resistance of $\rho_{M g H 2}=10^{5} \Omega \mathrm{nm}$ in hydrided $\mathrm{Mg}$ film, compared to bulk $\mathrm{MgH}_{2}$ or thin films that were prepared in the $\mathrm{MgH}_{2}$ phase. Similar results were found in this work. While no specific resistance can be given, the overall resistance of hydrided films is in the $10^{0} \Omega$ range, which is much lower than expected for the insulating magnesiumdihydride. The explanation of Giebels et al. was that grain boundaries act as conducting paths [101. Similar results have been found in $\mathrm{TiO}_{2}$. $\mathrm{TiO}_{2}$ is a similar good insulator as $\mathrm{MgH}_{2}$ and has the same rutile structure. Demetry et al. showed that grain boundaries improve the conductivity in $\mathrm{TiO}_{2}$ [106]. This gives an explanation for the change in resistance with changing hydrogen pressure seen in figure 6.4. Hydrogen is taken up by the 


\section{Global discussion of the experimental results}

grain boundaries if the hydrogen pressure surrounding the sample is increased 13 . Furthermore, the grain boundaries act as conducting paths through the thin films. The increased hydrogen concentration in the grain boundaries therefore decreases the conductivity of the grain boundary network, resulting in an increase of the overall resistance.

This explanation can be confirmed by the time scale of the effect. The increase and decrease of the resistance upon increasing or decreasing the hydrogen pressure are the result of the diffusion of hydrogen into and out of the grain boundary network. It can be evaluated similar to the general evaluation of a resistance measurement presented in chapter 3.3.3. However, the hydride volume fraction is not changed anymore, as the whole sample is already hydrided. Therefore, instead of evaluating the change of $\phi_{M g H 2}$, the change of the relative resistance $R / R_{0}$ is taken as a measure for the uptake over time $M / M_{\infty}$. Also, the diffusion length equals the film thickness, because the whole film is hydrided. Finally, diffusion from a limited volume does not seem to be a reasonable model in these cases. The reason for this is that not much hydrogen is taken up (no change in pressure is measurable). Instead a constant surface concentration is assumed (see 3.7 in chapter 3.2.2). Using all of this, three diffusion coefficients were evaluated. One for sample IBS-D1 (shown in figure 4.9) and two for sample IBS-E1 in figure 6.4 (one was evaluated from the first pressure increase to $100 \mathrm{hPa}$ and one from the following decrease to $7 \mathrm{hPa}$ ). All three are plotted in figure 6.5 as open red circles, so they can be compared with the other diffusion coefficients already shown before in figure 5.5. As said before, all of the other diffusion coefficients are grain boundary diffusion coefficients. This explains why the newly determined values fit well to the others, as they are also diffusion coefficients for the diffusion of hydrogen in the grain boundary network.

For sample IBS-D1 the value is larger than the other diffusion coefficients evaluated for the sample. The value is still inside the variance of all samples. However, it may be that the changes in hydrogen concentration change the diffusion coefficient of the grain boundary. This effect is well known for hydrogen bulk diffusion [232]. Overall, the resistance increase can be explained well by the uptake of hydrogen into grain boundaries. This allows the evaluation of additional grain boundary diffusion coefficients, which fit well to the values discussed above. It also explains the fact that the resistance increase does not result in a relevant change in the calculated hydride volume fraction $\phi_{M g H 2}$. The change is purely due to a reduction in the conductivity of the grain boundary network and not because of a phase transformation.

The extended decrease of the resistance found in sample IBS-D1 and IBS-E1 is not easily explainable. As said above, it does not seem to depend on a increase of the surrounding hydrogen pressure, but seems to happen after the sample is

\footnotetext{
${ }^{13}$ An increased hydrogen concentration in the grain boundaries results in a change in the hydrogen grain boundary segregation after the definition in equation 2.22 .
} 




Figure 6.5.: Replot of figure 5.5 adding the grain boundary diffusion coefficients evaluated from the resistance increase and decrease after increasing or reducing the hydrogen pressure surrounding an already hydrided sample. The added diffusion coefficients are marked as open red circles.

hydrided. Furthermore, it does not seem to change $\phi_{M g H 2}$ to a relevant degree. Some reasons for the change can be excluded. It is probably not related to changes in the measurement setup, as for a four-point setup changes, e.g. in the contact of the sample, can be neglected. It seems reasonable that the change is related to a kinetic process of the sample. Adsorption, dissociation and absorption should be on much faster timescales than the period of over $8 \cdot 10^{6} \mathrm{~s}$ of the resistance drop. This can be concluded from the other measurements in this work which were diffusion limited for time periods up to $10^{5} \mathrm{~s}$. As they were limited by diffusion, the other kinetic steps had to be faster. Taking all of this into account, it seems reasonable that some diffusion process happens on long times scales. Calculating a diffusion coefficient as $D=1 / d \cdot L^{2} / t$ allows the elimination of additional processes. It may be that the change in resistance is an effect of the Pd layer. However as it is only $35 \mathrm{~nm}$ thick this seems unreasonable (see table B.1 in appendix B.1.1). The calculated $D \approx 1.5 \cdot 10^{-21} \mathrm{~m}^{2} \mathrm{~s}^{-1}$ is much smaller than any diffusion coefficient of hydrogen in palladium or palladiumhydride known from literature. Flanagan and Oates e.g. give a diffusion coefficient in the range $10^{-10} \mathrm{~m}^{2} \mathrm{~s}^{-1}$ to $10^{-11} \mathrm{~m}^{2} \mathrm{~s}^{-1}$ for both phases [233]. Taking the $\mathrm{MgH}_{2}$ film thickness (about $600 \mathrm{~nm}$ ) as diffusion length, a diffusion 


\section{Global discussion of the experimental results}

coefficient in the range of $4.5 \cdot 10^{-19} \mathrm{~m}^{2} \mathrm{~s}^{-1}$ can be calculated. This is at the lower range of the grain boundary diffusion coefficient measured above. However, it is unclear which diffusion process of hydrogen in the grain boundaries would lead to a lowering of the overall resistance. Above, the opposite effect was ascribed to hydrogen diffusion in grain boundaries.

Overall, three possible explanations for the resistance drop will be discussed below. Other explanations are possible but at the given moment these three seem to fit the measurements the best. First, the diffusion process may be the diffusion of hydrogen from the grain boundaries into the grain. As said before, $\mathrm{MgH}_{2}$ does not take up further hydrogen. However, the grains do not have to be hydrided fully. Depending on the number of nuclei in a single grain, a shell of hydride can form around a magnesium core. Thereafter, hydrogen could diffuse through this shell to the center. This diffusion would be grain diffusion of $\mathrm{H}$ in $\mathrm{MgH}_{2}$, which is expected to be in the range of $10^{-29} \mathrm{~m}^{2} \mathrm{~s}^{-1}$. The grains in the film have a diameter of about $15 \mathrm{~nm}$ (see table 4.214 . Assuming a diffusion distance of about $5 \mathrm{~nm}$ this gives a diffusion coefficient of $3.1 \cdot 10^{-25} \mathrm{~m}^{2} \mathrm{~s}^{-1}$. This is still higher than the literature value, but as said before, the process was still ongoing when the measurement was ended, therefore the calculated value is only a maximum. While this scenario explains the long timescale it leaves some open questions. First of all, it is unclear why a hydride formation in the center of some grains leads to a reduction in resistance. The hydride has a higher resistance, but also it seems unreasonable that the core influences the global resistance, as the main conduction happens in the grain boundary network. If the grain boundaries loose hydrogen to the grains this could result in a lower resistance. However, because the sample is still in an hydrogen atmosphere the grain boundaries should be able to take up the hydrogen they loose to the grains 15 . Second of all, it is unclear how the shell would form, as the ETEM experiment seems to indicate that the already formed hydride creates the nanocrystalline structure.

The second possibility would be a recrystallization process. The nanocrystalline grain size in the ETEM sample was found to be slightly smaller than in the samples studied after a long time (compare table 4.2). This may indicate that the grains grow after the nanocrystalline structure is formed. However, grain growth would decrease the grain boundary density, which decreases the density of the conductive paths. Less conduction paths should increase the resistance, not decrease it. Furthermore, grain growth generally is described by diffusion through the grain boundaries [160]. Therefore, a similar diffusion coefficient as discussed above would be expected. However, the diffusion length between two grains is about $1 \mathrm{~nm}$. This gives a diffusion coefficient of about $D \approx 1.25 \cdot 10^{-24} \mathrm{~m}^{2} \mathrm{~s}^{-1}$, which is much lower than any value measured and discussed above.

\footnotetext{
${ }^{14}$ the samples were already hydrided once before, therefore it seems reasonable that the nanocrystalline structure is already formed.

${ }^{15}$ The diffusion from the hydrogen atmosphere was shown above to be much faster than the process discussed here.
} 
Finally, Nowotny et al. described long-term changes in the conductivity of titaniumdioxide [234]. During prolonged oxidation of $\mathrm{TiO}_{2}$, they found diffusion of oxygen on short time scales and afterwards a second long-term process they ascribed to the formation and diffusion of $\mathrm{Ti}$ vacancies. The effect found in this work may be of a similar origin. It does make sense that the diffusion of $\mathrm{Mg}$ defects is similarly slow in the $\mathrm{MgH}_{2}$ as the $\mathrm{H}$ diffusion. Further, additional defects may improve the conductivity of the $\mathrm{MgH}_{2}$ leading to a reduction of the overall resistance. Overall, there are several possible explanations for the long-term resistance drop found in this work. Further research is necessary to study this. The only definite result is that it occurs on very large time scales.

a)
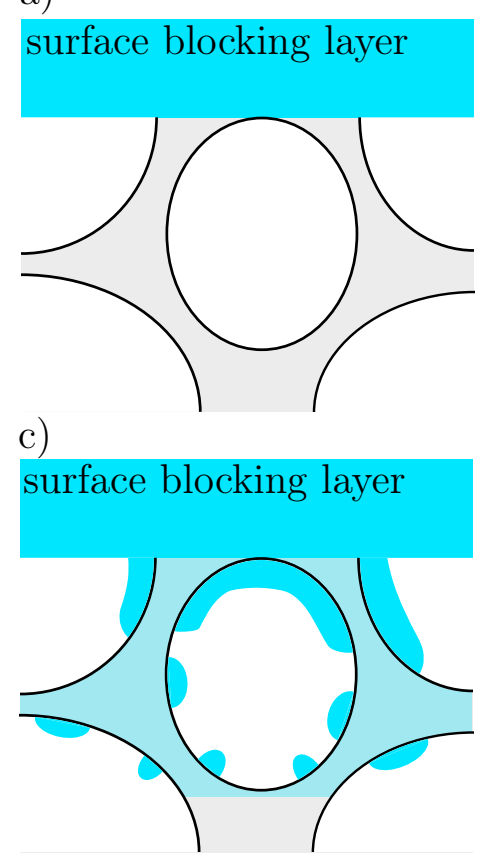

b)

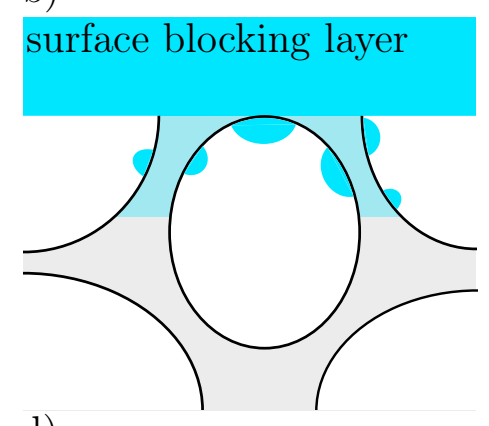

d)

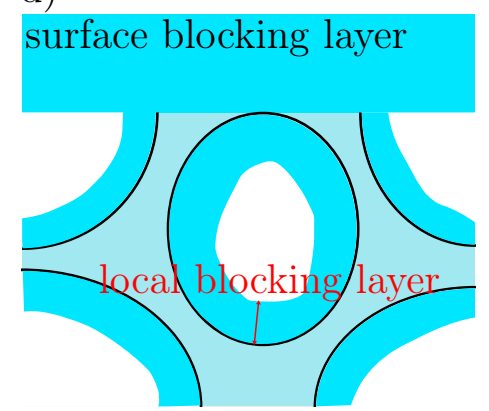

Figure 6.6: Principal of the local blocking layer: a) Magnesiumdihydride has formed a blocking layer on the surface, but under it magnesium grains (white) and grain boundaries (gray) remain. b) Hydrogen continues to diffuse through the grain boundaries in the hydride (not shown) and forms new hydrides in the magnesium grains. c) The new hydrides start to form a closed layer in each grain. d) In the end, each grain has formed a closed hydride shell (called local blocking layer) or is fully hydrided.

To put it in a nutshell, the results of this chapter allow to imagine an improved hydrogen storage material on the basis of magnesium. As shown in section 6.3.1 no dependence on the system diffusion on the grain size is found. On the one side, this reaffirmed the result of chapter 6.2 that hydrogen diffusion in magnesiumdihydride is described by Harrison regime $\mathrm{C}$. On the other side, grain and particle sizes are often reduced to improve the kinetics of the system (see chapter 2.1.4). While the grain size does not influence the diffusion ${ }^{16}$, it does improve the overall hydrogen density the system reaches. The reason for this is the blocking layer discussed before in the literature [24, 44, 43]. The blocking layer should not only be considered on the surface, as it was done most of the time in the literature. A local blocking layer in each grain needs to be discussed, because of the very low diffusion coefficients

\footnotetext{
${ }^{16}$ which limits the hydride formation
} 


\section{Global discussion of the experimental results}

in $\mathrm{MgH}_{2}$ crystals (see discussion in chapter 6.2 and chapter 2.1.4). The principle is schematically shown in figure 6.6. In figure 6.6 a), the surface blocking layer from the literature is shown (it also contains grains and grain boundaries which are not drawn for simplification purposes). Under the surface blocking layer $\mathrm{Mg}$ grains (in white) and grain boundaries (in gray) remain. Hydrogen diffuses through the grain boundaries of the surface blocking layer as the FEM simulation showed (see section 6.2). This leads to the formation of additional hydride in the grain as shown in figure 6.6 b). The influence of stress is not taken into account for this simple picture. For the thin films studied in this work it was shown in chapter 6.1 that the formation of hydride changes the grain morphology because of the high stresses in the samples. However, this is not expected to be as significant in bulk samples as they can expand much more freely. In these cases the new hydrides in the grains will begin to form closed layers of hydride, as shown in figure 6.6 c). In the end, this can lead to a $\mathrm{MgH}_{2}$ shell around a Mg core, as shown in figure 6.6 d). The shell is the local blocking layer mentioned above. Once the local blocking layer is formed, the magnesium in the center will not be reached by hydrogen in any relevant time period. Even if the shell is only $10 \mathrm{~nm}$ thick it will take over 300 years for hydrogen to diffuse through it at room temperature ${ }^{17}$. Hence, the local blocking layer is the reason that the sample will not reach its gravimetric limit of $7.6 \mathrm{wt} \%$ [19, 20, 21]. For larger grains not all magnesium can be hydrided because of the formation of local blocking layers in the grains. Unlike for the surface blocking layer, no apparent fast diffusion paths allow to bypass the very low hydrogen diffusion in $\mathrm{MgH}_{2}$. Therefore, because of the local blocking layer, it is important to reduce the grain size in samples. At some point a decreasing grain size will lead to hydride formation in the whole grain, thereby reaching its gravimetric limit.

H. Uchida calculated the average distance $l$ of hydride nuclei in thin films for two pressures (see figure 2.5 in chapter 2.1.4). For a pressure of $20 \mathrm{hPa}$ a distance of $l=680 \mathrm{~nm}$ was given and for a pressure of $200 \mathrm{hPa}$ a distance of $l=400 \mathrm{~nm}$ [76, 44]. This allows estimating the maximal grain size to fully hydride a grain. A three-dimensional grain should always be able to fully hydride if it has three or less nuclei 18 . Therefore, the maximum nuclei density $N_{\text {grain }}$ to fully hydride a grain is equal to:

$$
N_{\text {grain }}=\frac{3}{4 \pi r_{\text {grain }}^{2}}
$$

This assumes 3 nuclei and a grain radius of $r_{\text {grain }}$. The average nuclei density $N_{\text {ave }}$ in thin films can be calculated from their distance $l$ as calculated by Uchida :

$$
N_{\text {ave }}=\frac{1}{\pi(l / 2)^{2}}
$$

\footnotetext{
${ }^{17}$ This assumes a diffusion coefficient of $10^{-29} \mathrm{~m}^{2} / \mathrm{s}$ (see figure 6.2 )

${ }^{18}$ For more nuclei the grain may still fully hydride, depending on the position of the nuclei.
} 
By setting $N_{\text {grain }}=N_{\text {ave }}$, the maximum grain radius can be calculated for which the whole grain can form $\mathrm{MgH}_{2}$. For the two pressures measured by Uchida the two corresponding radii are approximately $290 \mathrm{~nm}$ (for a $\mathrm{H}$ loading pressure of $20 \mathrm{hPa}$ ) and $170 \mathrm{~nm}$ (for for a $\mathrm{H}$ loading pressure of $200 \mathrm{hPa}$ ). This shows how difficult it is to fully hydride a bulk sample, which typically has grain sizes in the micrometer range and above.

While reducing the grain size allows a sample to reach its maximal gravimetric density, it does not increase the sample kinetics as shown in section 6.3.1. To improve the system kinetics the grain boundary diffusion has to be improved. One possible way to achieve this was shown in section 6.3.2. The addition of iron to magnesium was found to increase the grain boundary diffusion coefficients. In the literature other materials like aluminum, gallium, indium and cerium were found to possibly have the same effect [143, 23, 151, 231. Further research is needed to find the element or combination of elements and their optimal concentration to reach high grain boundary diffusion coefficients. Overall, a possible storage medium would combine small grain sizes (e.g. produced by ball milling) with a low concentration of additives that improve the grain boundary diffusion. The result would hopefully posses fast kinetics and a gravimetric density close to pure magnesium. 



\section{Summary and outlook}

The practical application of magnesium as a hydrogen storage material is limited by its kinetics [20, 23, 25, 29]. To be more exact, it is the diffusion of hydrogen through the magnesiumhydride phase that slows down the overall kinetics (the so called blocking layer effect) [41, 42]. Grain boundaries may offer fast diffusion paths through the $\mathrm{MgH}_{2}$ and thereby improve the formation behavior. Therefore, this thesis aimed to unravel the role of grain boundaries on the hydride formation in $\mathrm{Mg}-\mathrm{H}$. This includes deducing the contribution of grain boundaries on the overall diffusion kinetics in the dihydride phase, which is limited by the blocking layer. Furthermore, microstructural changes are studied to investigate the influence of these changes on the kinetic and thermodynamic behavior. Finally, the impact of a ternary compound $(\mathrm{Fe})$ on the overall diffusion kinetics is investigated as a possibly improvement.

To study the influence of grain boundaries, grain boundary rich thin films were prepared. Two preparation techniques were used: ion beam sputtering (IBS) and pulsed laser deposition (PLD) (see chapter 3.1). This allowed to prepare different grain morphologies and compare their influence on the overall diffusion. The diffusion was measured by three different techniques: X-ray diffraction (see chapter 3.5), gas volumetry (see chapter 3.2) and resistance measurements (see chapter 3.3). For the resistance measurement technique an analytic model was developed that allows evaluating the volume fraction of the $\mathrm{MgH}_{2}$ at a given time from the resistance. The volume fraction allows the determination of the diffusion constant, as well as to evaluate the limiting kinetic process of the measurement. Hence, it can be confirmed that a given measurement is limited by diffusion and not by another limiting kinetic process (e.g. hydrogen absorption or phase transformation). The experimental techniques allow to measure the overall system diffusion coefficient of the thin films in dependency of different parameters like the grain size and gas pressure. One of the compared parameters was the concentration of iron additives in the thin films. Iron is known not to solve in magnesium (see reference [69]). Therefore, it may segregate in grain boundaries and change the grain boundary diffusion. To study the influence of the grain boundary diffusion and volume diffusion on the overall system diffusion, finite-element simulations (FEM) were performed (see chapter 3.6).

The grain morphology of the thin films prepared was studied at different states of the hydrogen loading experiments (see chapter 4). X-ray diffraction (XRD) and 


\section{Summary and outlook}

transmission electron microscopy (TEM) were used to study the grain morphology before and after hydrogen loading. Before hydrogen loading the IBS films showed large columnar grains with in-plane grain sizes of about $60 \mathrm{~nm}$. Thicker films showed somewhat larger in-plane grain sizes. The out-of-plane grain size seems to be equal to the film thickness, with the exception of some smaller grains at the substrate/film interface. After hydrogen loading (and unloading), the grains were found to be equiaxed with grain sizes of around $15 \mathrm{~nm}$. Before hydrogen loading the PLD films showed mostly equiaxed grains with grain sizes of about $10 \mathrm{~nm}$, along with some larger grains similar to the grains of the IBS films. After hydrogen loading the large grains were transformed in smaller equiaxed grains similar to what happened in the IBS films. The other grains, which were already small and equiaxed before hydrogen loading, may slightly reduce their grain size during the hydride formation However, this is not a clear outcome. Overall, the formation of the magnesiumdihydride seems to lead to a strong nanocrystalline structure.

This nanocrystallisation was confirmed by an in situ TEM and EELS study. A TEM lamella of an IBS film was loaded in the TEM. It was found that a nucleus forms at the thinnest point of the lamella and grows half-spherical afterwards. It was shown that the growing $\mathrm{MgH}_{2}$ forms the nanocrystalline structure. The reason for the formation of the nanocrystalline structure is believed to be the influence of high stresses in the thin films. It was shown in the literature that high stresses form in thin films (in general and for similar $\mathrm{Mg}$ thin films), because the in-plane expansion is prevented by the substrate [76, 88]. This explains the formation of the nanocrystalline structure as the high stresses result in plasticity, which strongly changes the grain structure. The nucleation and growth behavior found in the TEM lamella has been predicted by Uchida et al. [44] in his description of the development of the blocking layer (see figure 2.5 in chapter 2.1.4). However, in this work it was found that the hydride additionally grows fingers along the magnesium/palladium interface. This leads to a closed hydride layer on top of the film, blocking the film much earlier than the pure half-spherical growth would do.

The hydride can be unloaded by contact with air without the need of increased temperatures. However, the hydride decomposition does not reverse the nanocrystalline grain structure. The hydride decomposition stands in opposition to the thermodynamic equilibrium conditions of the bulk system, which would predict a decomposition at temperatures of about $577 \mathrm{~K}$ (see section 2.1.1). However, the hydrogen release was confirmed by resistance measurements, XRD and TEM measurements (see chapter 4.2). It is known that stresses change the thermodynamic behavior in thin films [84, 85, 86]. It was shown that high stresses reduce the critical temperature and thereby reduce the temperature necessary to dehydride a thin film. This explains the results of this work.

The finite-element simulations gave a better insight into the diffusion process in magnesiumdihydride containing grain boundaries. The FEM results in dependency 
of time and grain size were compared with the analytic descriptions given by Harrison 64. (see chapter 2.2.4). For diffusion coefficients in volume and grain boundary of similar order of magnitude it was found that the grain size is of major importance, as it strongly influences which analytic description needs to be applied (see chapter 6.2.1). It further appears that the Harrison regime $\mathrm{A}$ is applicable for larger grain sizes than the theory would predict. For a large ratio $\Delta=D_{G B} / D_{V}>1000$ of the grain boundary diffusion coefficient $D_{G B}$ and the volume diffusion coefficient $D_{V}$, Harrison regime $\mathrm{C}$ describes the FEM simulations the best. This regime is completely governed by the grain boundaries and volume diffusion can be ignored. The large scatter found in the literature regarding $\mathrm{H}$ diffusion in $\mathrm{MgH}_{2}$ can be explained with regards to the results of the FEM simulations. The diffusion in crystals of $\mathrm{MgH}_{2}$ (with no influence of grain boundaries) is found to be in the range of $D \approx 10^{-29} \mathrm{~m}^{2} \mathrm{~s}^{-1}$ at room temperature. Measurements in samples containing grain boundaries showed values of $10^{-16} \mathrm{~m}^{2} \mathrm{~s}^{-1}$ to $10^{-20} \mathrm{~m}^{2} \mathrm{~s}^{-1}$. The large difference can be explained by diffusion in Harrison regime $\mathrm{C}$, which means that the experiments in samples with grain boundaries measure only the grain boundary diffusion coefficient $D_{G B}$. This explains also the larger scatter for measurements containing grain boundaries. The different samples are expected to have different grain structures and grain boundary structures. Different grain boundaries have different grain boundary diffusion coefficients $D_{G B}$, which are measured in the diffusion experiments in regime C.

The evaluation of the limiting kinetic process confirmed that almost all measurements investigated are limited by a diffusion process (see chapter 5.1). The diffusion coefficients measured by different techniques during the same measurement fit well to each other. Overall an average diffusion of $D_{G B}=6.9_{-6.5}^{+140} \cdot 10^{-18} \mathrm{~m}^{2} \mathrm{~s}^{-1}$ was found for all samples (see chapter 5.2). No dependence on the grain size was found, which confirms the finding that the measured diffusion coefficient is the grain boundary diffusion coefficient (see chapter 6.3.1). Increasing or decreasing the grain boundary density (by changing the grain size) does not change the speed of transport in the grain boundary, only the amount of transported diffusant. This explains why the structural changes during the hydride formation do not influence the grain boundary diffusion coefficient. An apparent dependence on the loading pressure was found. However, it can be shown that this dependence is actually a dependence on the iron content in the thin films (see chapter 6.3.2). Iron seems to increase the grain boundary diffusion coefficient. It is most probable, that iron segregates at the grain boundaries and thereby leads to changes in the grain boundary structure. These changes seem to lead to an improved grain boundary diffusion of hydrogen.

Further insight, into the behavior of the $\mathrm{MgH}_{2}$ grain boundaries, was gained by adding additional hydrogen into the hydrided thin films (see chapter 6.3.3). This leads to an increase of the measured resistance. The effect can be explained with regards to the work of Westerwaal et al. and Demetry [101, 106]. Westerwaal et al. proposed that the low resistance found in hydrided $\mathrm{MgH}_{2}$ films $\left(\mathrm{MgH}_{2}\right.$ is an 


\section{Summary and outlook}

insulator) is the result of the grain boundaries, which act as a conducting network. Demetry et al. confirmed that grain boundaries can improve electric conductivity for $\mathrm{TiO}_{2}$, another insulator with the same rutile crystal structure as $\mathrm{MgH}_{2}$. The resistance increase is therefore interpreted to be a result of additional hydrogen taken up into the grain boundary network, which lowers the conductivity of the network. A diffusion coefficient can be evaluated during this additional uptake. It was found to be the same as for the other measurements, which confirms that for both cases the inward diffusion of hydrogen in the grain boundary network is measured. For long times a drop of the resistance of the films can be found. This is most likely the result of formation and diffusion of magnesium defects in accordance to a similar result found in $\mathrm{TiO}_{2}$ by Nowotny et al. [234]. The origins of the process need to be confirmed, but it happens on very slow time scales, meaning the process has a diffusion coefficient of below $10^{-25} \mathrm{~m}^{2} \mathrm{~s}^{-1}$.

To conclude, the results of this work show how magnesium can be improved for the application as a hydrogen storage material. The kinetics of magnesium is limited by the diffusion through magnesiumdihydride. This work showed that this process is dominated by the diffusion through grain boundaries. This leads to two changes necessary for an efficient storage medium. First, the grain and particle sizes need to be small. This will lead to a high percentage of hydrided material before a closed hydride layer (or shell) is formed. Further, the grain boundary diffusion needs to be improved to reduce the time necessary to hydride the magnesium left under the $\mathrm{MgH}_{2}$ surface layer. This work indicates one way how this may be ensured. The addition of iron increases the hydrogen grain boundary diffusion. Further research is needed in finding the best additive to increase the grain boundary diffusion and the optimal concentration of the additive. 
Appendices 



\section{A. Additions to Experimental methods}

The appendix collects some additions to the experimental methods presented in chapter 3.2. Figure A.1 shows the two background measurements of the gas volumetry setup without sample or resistance stage (measured by F.Jung). The drop can probably be attributed to a drift of the membrane in the baratron pressure gauges and is corrected in the measurements of the diffusion coefficients.

Figure A.2 shows how $Z_{1}$ and $q_{1}^{2}$ develop in dependence on $\lambda$. Both values converge quickly for $\lambda>10$. As most measurements done in this work had $\lambda \approx 100$ no large error to measurements is introduced by the error of $\lambda$. This is of importance for the measurements of diffusion coefficients as described in chapter 3.2 and chapter 3.3 .
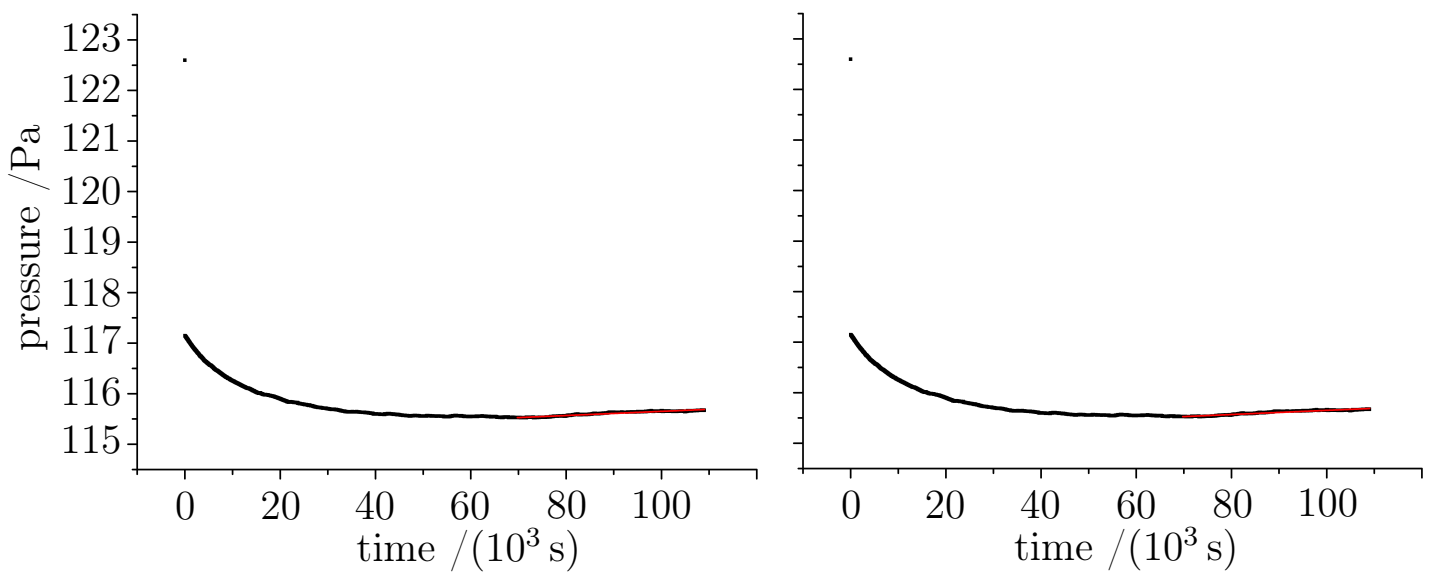

Figure A.1.: Pressure drop during two hydrogen loading experiments with $122 \mathrm{~Pa}$ without sample or resistance stage. The red line is the pressure increase, because of leakage 
A. Additions to Experimental methods

Figure A.2: Change of $q_{1}^{2}$ and $Z_{1}$ with increasing values of $\lambda$. Both values converge quickly for values of $\lambda>10 . q_{1}^{2}$ converges against 2.4674 and $Z_{1}$ against 0.81057 .

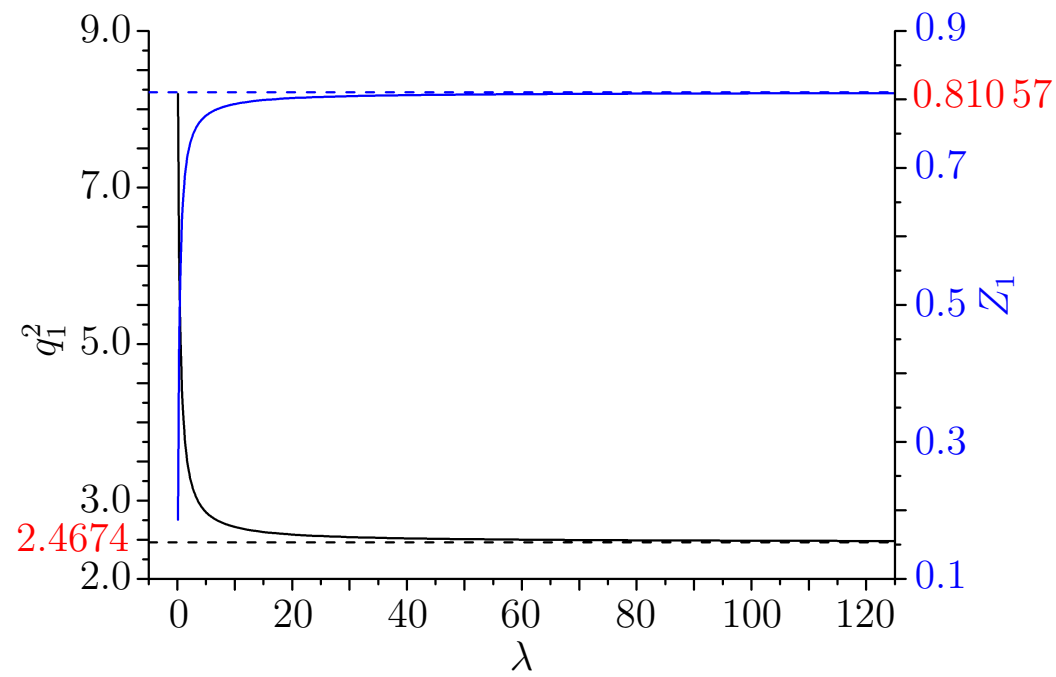




\section{B. Additional results}

The following appendix collects some additional results of this work. While interesting, they are not essential for answering the main research question: How do grain boundaries influence the diffusion of hydrogen through the magnesiumdihydride. First, some additional information about the studied thin magnesium film are given. The film thickness of the magnesium and palladium layer is collected in section B.1.1. Afterwards, additional XRD measurements are discussed in section B.1.2. Finally, a palladium-magnesium interlayer is presented, which was identified in the IBS-ETEM sample. The second part of the chapter presents additional results from the measurements of diffusion coefficients. First, additional data from the evaluation of the limiting kinetic process is presented (section B.2.1) and later on additional results regarding the diffusion coefficients (section B.2.2). 


\section{B.1. Structural characterization of $\mathrm{Mg}$ thin films upon hydrogen loading}

\section{B.1.1. Film thickness changes upon hydrogen loading}

Table B.1 gives the film thicknesses of the Mg thin films and their Pd capping layer. The values were measured by TEM and, in addition, by a Dektak 150 profilometer (values in brackets). The values before and after hydrogen loading are not measured on the same sample but on different samples of the same batch. The values of table B.1 are plotted in figure 4.12 in chapter 4.3 .

Table B.1.: Film thicknesses of the thin magnesium films and their palladium capping layers. The values were measured by TEM cross sections. In some cases additional measurements were performed using a Dektak 150 profilometer. The values measured in this manner are given in brackets.

\begin{tabular}{|c|c|c|c|c|}
\hline \multirow[t]{2}{*}{ Batch } & \multicolumn{2}{|c|}{$\begin{array}{l}\text { Before hydrogen loading } \\
\qquad /(\mathrm{nm})\end{array}$} & \multicolumn{2}{|c|}{$\begin{array}{l}\text { After hydrogen loading } \\
\qquad /(\mathrm{nm})\end{array}$} \\
\hline & magnesium & palladium & magnesium & palladium \\
\hline \multirow[t]{2}{*}{ IBS-A } & $638 \pm 27$ & $31 \pm 4$ & $608 \pm 31$ & \\
\hline & $(534 \pm 55)$ & & $(688 \pm 92)$ & \\
\hline \multirow[t]{2}{*}{ IBS-B } & $623 \pm 9$ & $28 \pm 5$ & $636 \pm 5$ & $20 \pm 3$ \\
\hline & $(560 \pm 20)$ & & $(458 \pm 5)$ & \\
\hline IBS-C & $(4330 \pm 95)$ & & & $28 \pm 4$ \\
\hline \multirow[t]{2}{*}{ IBS-D } & $403 \pm 8$ & $34 \pm 3$ & $471 \pm 5$ & $36 \pm 5$ \\
\hline & $(347 \pm 20)$ & & & \\
\hline \multirow[t]{2}{*}{ IBS-E } & $670 \pm 14$ & $45 \pm 3$ & $838 \pm 32$ & $52 \pm 6$ \\
\hline & $(510 \pm 52)$ & & & \\
\hline IBS-F & $660 \pm 8$ & $40 \pm 3$ & $680 \pm 18$ & $39 \pm 5$ \\
\hline PLD-noPd & $(140 \pm 30)$ & & & \\
\hline PLD-Pd & $86 \pm 4$ & $33 \pm 4$ & $188 \pm 9$ & $37 \pm 4$ \\
\hline
\end{tabular}




\section{B.1.2. XRD measurements of $\mathrm{Mg}$ thin films}

Additional XRD measurements were done on the PLD and IBS Mg thin films. The results are of general interest, but do not offer much insight into the influence of grain boundaries on the hydrogen diffusion. Therefore they are discussed here. Figure B.1 shows a XRD measurement of a PLD sample. In black a measurement directly after preparation is displayed, the grey curve belongs to a measurement after four months storage in air, while the blue curve belongs to a measurement, which was loaded with hydrogen for three months. Many of the same features reported in chapter 4.1 about the IBS samples are found again (see figure 4.1). Marked by black dashed lines are the silicon (400) peak, the magnesium (002) peak and the palladium (111) and (200) peaks. All of these were discussed for the IBS sample presented before. The $\mathrm{Mg}$ peak is much weaker in this sample as the film is thinner and the grain size is smaller (see chapter 4.1). A sharp peak can be seen to smaller $2 \Theta$ values of the $\mathrm{Mg}$ peak. By comparison of the lattice distance values $l$ it can be identified as the formally forbidden (200) silicon peak (see equation 3.18 in chapter 3.5. The green line may correspond to a $(100) \mathrm{Mg}(\mathrm{OH})_{2}$ peak ${ }^{1}$. This would explain why it is only visible months after preparation. However, it forms even with a Pd capping layer and for the sample that spent the most time in vacuum or a hydrogen atmosphere. It is therefore probable that no closed hydroxide layer is formed, only single hydroxide nuclei. The red lines indicate peaks that cannot be described by known phases of magnesium, palladium, silicon, magnesiumoxide, magnesiumhydroxid or magnesiumhydride. This includes the magnesiudihydride $\gamma$-phase, which was described by Ham et al. to form in $\mathrm{Mg}$ thin films [71]. One possible explanation for the peak would be a palladium-magnesium phase. Several different phases are known (see figure 2.4 in chapter 2.1.4) and it has been confirmed in another sample studied in this work (compare appendix B.1.3). The peaks are not visible directly after preparation. While most literature found an intermixing of $\mathrm{Mg}$ and $\mathrm{Pd}$ at high temperatures, it can not be excluded that alloy phases form at room temperature. Singh et al. found a formation during preparation by PLD [37]. This cannot be confirmed here. However, as discussed in chapter 6.1.1, differences in the preparation of Singh et al. and the films studied in this work exist. The exact phase of the possible interlayer could not be identified, making further research necessary.

To confirm that the new peaks discussed above are not only a result of the PLD preparation figure B.2 shows two IBS samples four and a half months after preparation. The black curve displays data from a sample stored in air, while the blue curve displays data from a sample loaded with hydrogen, which spent most of the time between the loading steps in vacuum. The typical $\mathrm{Mg}, \mathrm{Pd}$ and Si peaks are visible again (marked by dashed black lines). The influence of the hydrogen loading can be seen here. The Pd and Mg peaks shift to the right, indicating smaller lat-

\footnotetext{
${ }^{1}$ Compare chapter 2.1 .4 for a description of the formation of the $\mathrm{MgO} / \mathrm{Mg}(\mathrm{OH})_{2}$ layer
} 


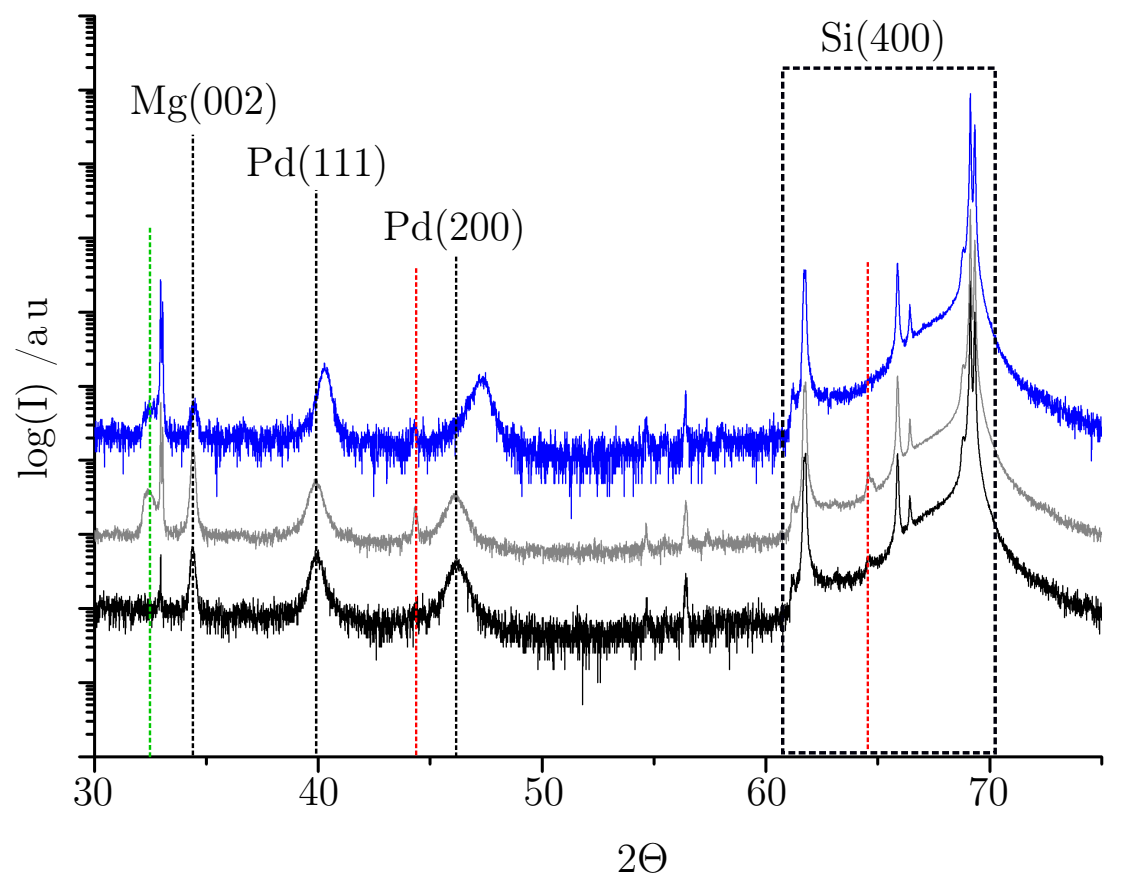

Figure B.1.: XRD measurement of PLD prepared magnesium thin films after preparation (black curve), four months after preparation (gray curve) and after hydrogen loading for three months (blue curve). The black lines mark the magnesium (002), palladium $(111) /(200)$ and silicon (400) peaks already shown before in figure 4.1. The red lines mark peaks possibly originating from the palladium-magnesium interlayer. They are discussed further in the text. The green line marks a peak that may be attributed to a (100) $\mathrm{Mg}(\mathrm{OH})_{2}$ orientation.

tice distances. At the same time the $\mathrm{Mg}$ peaks become much weaker. Both effects can be explained by the change in the Mg microstructure. As discussed in chapter 6.1, the grain structure becomes much more nanocrystalline after hydrogen loading. This leads to weaker magnesium XRD peaks, as the overall grain volume is reduced. The decrease of the grain size is a consequence of the lattice expansion of the thin film and the resulting plasticity. This plasticity strongly reduces the stresses in the film. The shift of the peaks shows this decrease in the internal stress (for further information see reference [215]). A green dashed line marks the (100) $\mathrm{Mg}(\mathrm{OH})_{2}$ peak already found in figure B.1. A new peak marked by a magenta dashed line is found in the loaded sample. It could fit to $(002) \mathrm{Mg}(\mathrm{OH})_{2}$ peak or a (111) $\mathrm{MgO}$ peak. Because it is only visible in a sample that was in contact with hydrogen and it is found in combination with the $(100) \mathrm{Mg}(\mathrm{OH})_{2}$ peak it seems more probable to be a (002) $\mathrm{Mg}(\mathrm{OH})_{2}$ peak. It is unclear why the $\mathrm{Mg}(\mathrm{OH})_{2}$ peaks are only visible in the loaded sample. This was not the case for the PLD sample where a $(100) \mathrm{Mg}(\mathrm{OH})_{2}$ 
peak was found for samples aged in air as well as for samples aged in hydrogen. Several peaks of a possible palladium-magnesium interlayer are found again. They all seem to be weaker for the sample that was loaded with hydrogen. A similar, but weaker, trend is visible for the PLD samples in figure B.1. This may be a result of the change in microstructure for the samples loaded with hydrogen.

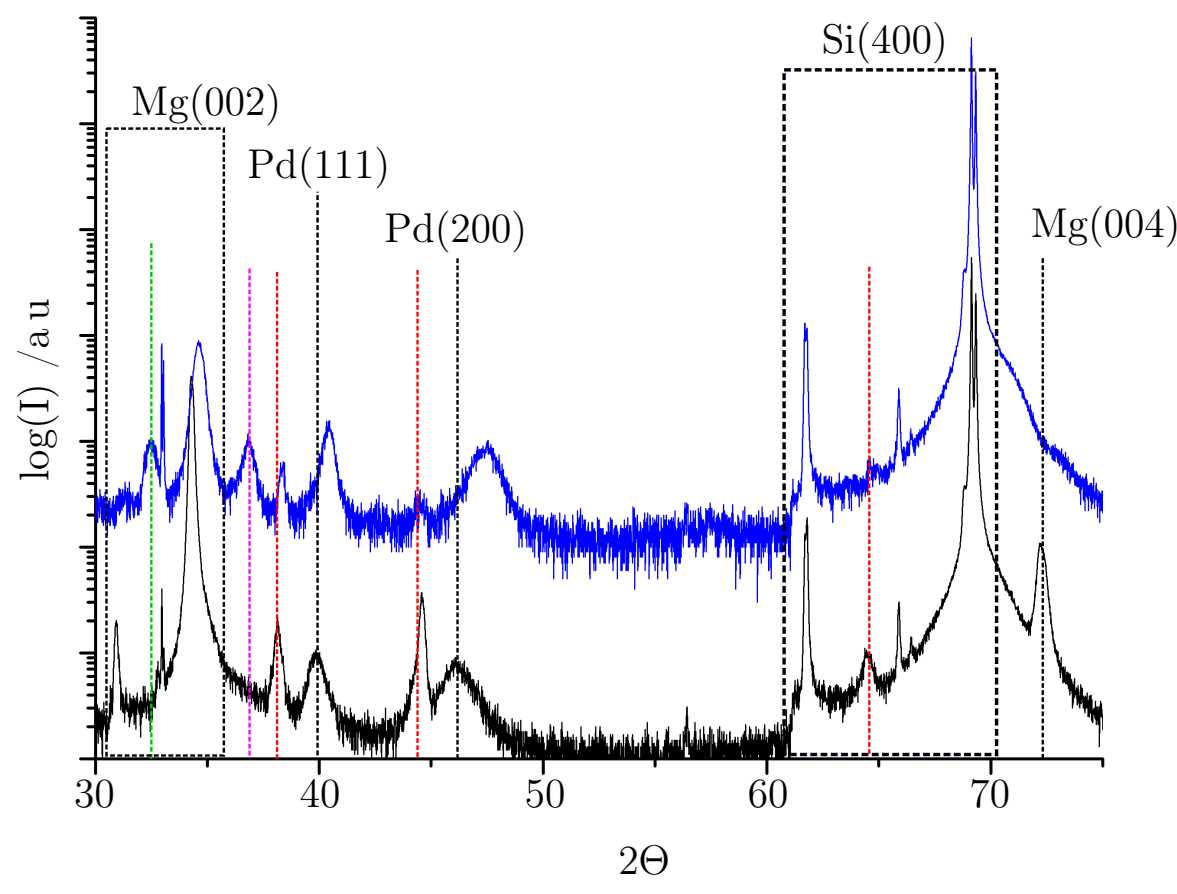

Figure B.2.: XRD measurement of IBS prepared magnesium thin films that were measured four and a half months after preparation. The sample characterized by the black curve is as-prepared, while the sample characterized by the blue curve was loaded with hydrogen. The black lines mark the magnesium (002), palladium $(111) /(200)$ and silicon (400) peaks already shown before in figure 4.1. The red lines mark peaks possibly originating from the palladium-magnesium interlayer. They are discussed further in the text. The green line marks a peak that may be attributed belong to a $(100) \mathrm{Mg}(\mathrm{OH})_{2}$ orientation. The magenta peak may belong to a $(002) \mathrm{Mg}(\mathrm{OH})_{2}$ orientation or to a (111) $\mathrm{MgO}$ orientation. 


\section{B.1.3. EELS measurements of a palladium/magnesium interlayer}

Appendix B.1.2 discussed a palladium-magnesium interlayer as the possible origin of some XRD peaks found in aged samples. This interlayer has been definitely identified in sample IBS-ETEM by EELS. Figure B.3 shows an EELS map of the interface between the magnesium thin film and the palladium capping layer. A layer of mixed composition with an approximate thickness of $10 \mathrm{~nm}$ could be identified between the two. The sample was prepared about a half a year before the experiments. It is unclear if the layer was present from the beginning or formed in the meantime. The XRD results of appendix B.1.2 indicate the latter.

Figure B.3: EELS map of the distribution of palladium and magnesium at their interface. An interlayer of $\mathrm{Mg}-\mathrm{Pd}$ forms at the interlayer, separating the pure magnesium thin film from the palladium cap.
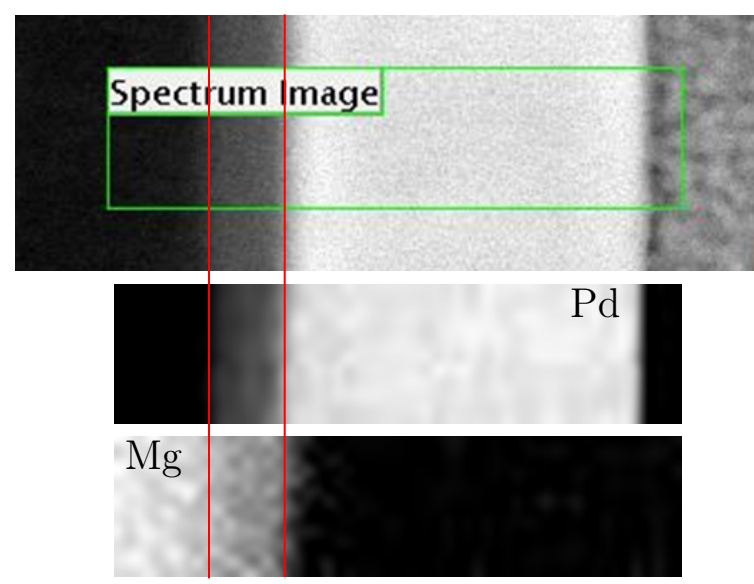


\section{B.2. Measurement results of diffusion coefficients of hydrogen in nanocrystalline $\mathrm{MgH}_{2}$}

\section{B.2.1. Evaluation of the limiting kinetic process}

\begin{tabular}{|c|c|c|c|c|}
\hline Sample & $\begin{array}{c}p_{H} \\
/(\mathrm{hPa})\end{array}$ & $\begin{array}{l}t_{90} \\
/(\mathrm{s})\end{array}$ & impingement parameter $\eta$ & JMAK regions \\
\hline IBS-A1 & 800 & 11500 & $\overline{5.6 \cdot 10^{10}}$ & 2 \\
\hline IBS-A2 & 300 & 3600 & $6.4 \cdot 10^{10}$ & 4 \\
\hline IBS-A3 & 500 & 1700 & $1.1 \cdot 10^{11}$ & 3 \\
\hline IBS-B1 & 50 & 11000 & $1.3 \cdot 10^{12}$ & 2 \\
\hline${\mathrm{IBS}-B 2^{a}}$ & 800 & 4000 & $5.4 \cdot 10^{11}$ & 1 \\
\hline IBS-D1 & 10 & 25000 & 1 & 1 \\
\hline IBS-D1 & 10 & 118000 & 1 & 1 \\
\hline IBS-E1 & 25 & 45000 & 1 & 2 \\
\hline IBS-E1 & 7 & 281000 & 1 & 2 \\
\hline PLD-noPd & 7 & 14500 & $2.2 \cdot 10^{11}$ & 1 \\
\hline PLD-noPd & 7 & 120000 & 1 & 2 \\
\hline PLD-Pd & 10 & 27300 & $1.2 \cdot 10^{11}$ & 1 \\
\hline PLD-Pd & 30 & 840 & $4.6 \cdot 10^{10}$ & 1 \\
\hline
\end{tabular}

Table B.2.: Overview of parameters for the evaluation of the limiting kinetic process. For each sample where the hydride volume fraction $\phi_{M g H 2}$ was evaluated the applied hydrogen pressure $p_{H}$ is given for the evaluated loading step. $t_{90}$ gives the time the sample needs to reach $90 \%$ of the maximum hydride volume fraction. The impingement parameter $\eta$ was evaluated by fitting equation 2.30 to the data. The final value gives the number of regions with different slope $n$ in a plot of $\ln \left(\ln \left(1 /\left(1-\phi_{M g H 2}\right)\right)\right)$ over $\ln (t)$ (compare equation 2.29 . For regions which cover more than $75 \%$ of $t_{90}$ additional evaluated parameters are given in table 5.1 .

a): The resistance measurement shows an unexplained dip during the measurement. However, the data was evaluated in the same way as all other samples

Table B.2 collects some additional results of the evaluation of the limiting kinetic factors. For all evaluated samples it gives the loading pressure and the time needed to reach $90 \%$ of the reached hydride volume fraction $\phi_{M g H 2}$. This time is a relevant parameter, as afterwards no significant changes in the resistance happen anymore. Additional the evaluated impingement parameter $\eta$ are give, for large $\eta$ the JMAK model can be applied to describe the kinetics. Finally, the number of regions with different slope in a JMAK plot are given. The final evaluation only uses the region covering the longest time period. The only exception is sample IBS-E1, as discussed in chapter 5.1 .

Figure B.4 shows the integrated reaction model of the Ginstling-Brounshtein model 


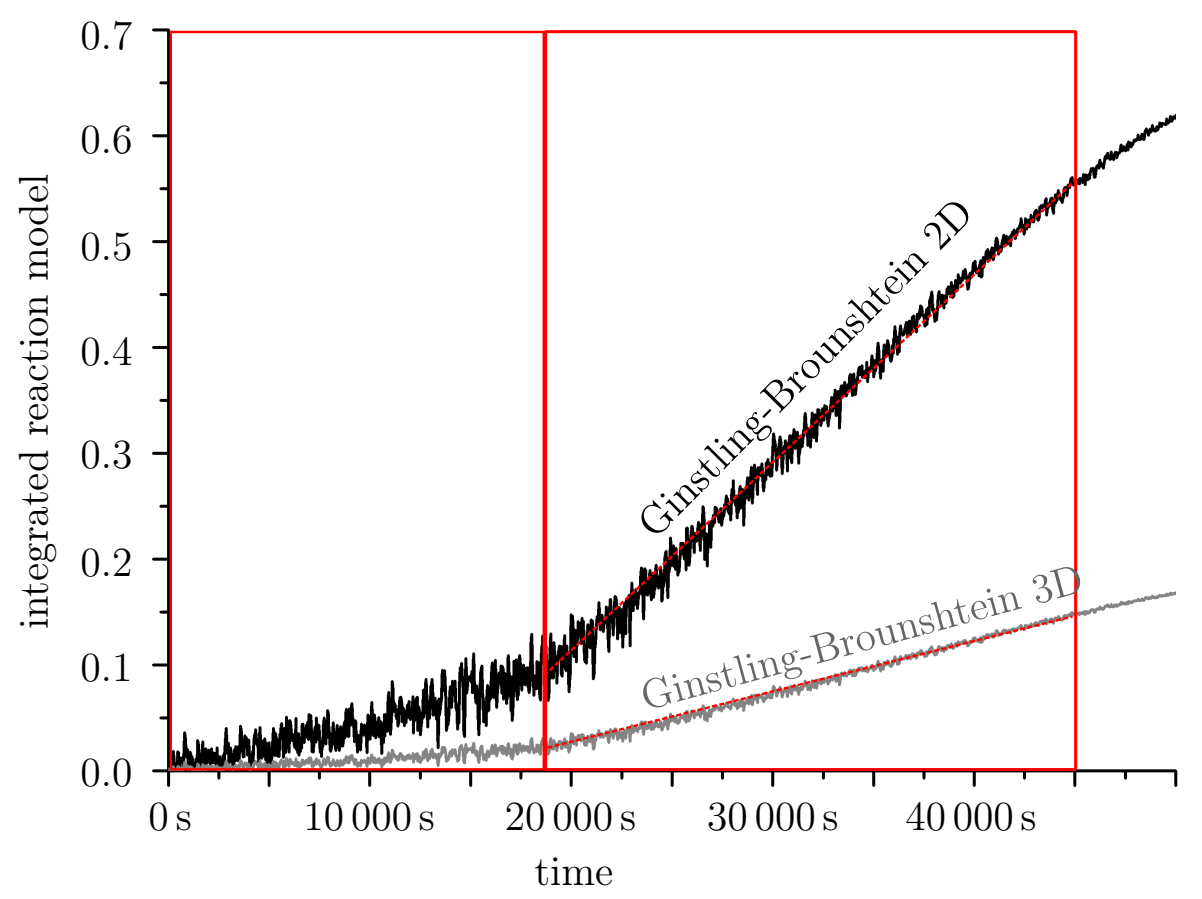

Figure B.4.: Comparison of integrated reaction model, evaluated by resistance measurement, of the Ginstling-Brounshtein model in 2D and 3D for sample IBS-E1 (first time loaded). The model in both dimensions fits better to the data than the CV model or Jander's model (see figure 5.2). This indicates that the kinetics is limited by diffusion in more than one dimension.

in $2 \mathrm{D}$ and $3 \mathrm{D}$ plotted as function of time (see chapter 2.3). The fit of these models for the second region (18000s to $45000 \mathrm{~s}$ ) is better than any other model tested. This indicates a diffusion limitation in two or three dimensions. 


\section{B.2.2. Results of overall system diffusion coefficient measurements}

This appendix collects some additional results of the diffusion coefficient measurements. Table B.3 collects all diffusion coefficients measured in samples that were found to be limited by diffusion (see chapter 5.1). Diffusion coefficients measured by the three used methods (gas volumetry, resistance measurement and X-ray diffraction) are given along with the sample name and the hydrogen loading pressure $p_{H}$.

Table B.3.: Overall system diffusion coefficients measured in this work. The first columns give the sample name and the applied loading pressure $p_{H}$. In the following the measured diffusion coefficients are listed. Three independent methods were used to measure diffusion coefficients. In situ XRD measurements $\left(D^{X R D}\right)$, gas volumetry measurements $\left(D^{G}\right)$ and resistance measurements $\left(D^{R}\right)$. Marked in red is the measurement which is limited by the diffusion process, but not in one dimension as discussed in section 5.1.

a): no new loading, the hydrogen pressure is increased from the previous step.

b): $D^{R}$ is not corrected with the background measurements

\begin{tabular}{l|llll}
\hline Sample & $\begin{array}{l}p_{H} \\
/(\mathrm{hPa})\end{array}$ & $\begin{array}{l}D_{\text {ove }}^{X R D} \\
/\left(\mathrm{m}^{2} \mathrm{~s}^{-1}\right)\end{array}$ & $\begin{array}{l}D_{\text {ove }}^{G} \\
/\left(\mathrm{m}^{2} \mathrm{~s}^{-1}\right)\end{array}$ & $\begin{array}{l}D_{\text {ove }}^{R} \\
/\left(\mathrm{m}^{2} \mathrm{~s}^{-1}\right)\end{array}$ \\
\hline \hline IBS-A1 & 800 & $(4.3 \pm 2.2) \cdot 10^{-17}$ & & $(1.6 \pm 0.9) \cdot 10^{-17}$ \\
IBS-A2 & 300 & $(7.5 \pm 4.0) \cdot 10^{-17}$ & & $(4.6 \pm 2.4) \cdot 10^{-17}$ \\
IBS-A3 & 500 & $(1.5 \pm 0.8) \cdot 10^{-16}$ & & $(1.3 \pm 0.7) \cdot 10^{-16}$ \\
IBS-A5 & 300 & & $(1.3 \pm 0.7) \cdot 10^{-16}$ & \\
IBS-B1 & 50 & $(5.6 \pm 2.9) \cdot 10^{-17}$ & & $(1.5 \pm 0.8) \cdot 10^{-17}$ \\
IBS-B2 & 800 & $(7.4 \pm 4.2) \cdot 10^{-17}$ & & $(4.6 \pm 2.4) \cdot 10^{-17}$ \\
IBS-C1 & 300 & & $(6.2 \pm 3.2) \cdot 10^{-17}$ & \\
IBS-C2 & 100 & & $(7.2 \pm 3.7) \cdot 10^{-17}$ & \\
IBS-D1 & 10 & & $(2.2 \pm 1.2) \cdot 10^{-18}$ & $(2.6 \pm 1.4) \cdot 10^{-18}$ \\
& 10 & & $(7.3 \pm 3.7) \cdot 10^{-19}$ & $(1.2 \pm 0.6) \cdot 10^{-18}$ \\
& $100^{a)}$ & & & $(4.9 \pm 2.5) \cdot 10^{-17}$ \\
IBS-E1 & $25^{b)}$ & & $(6.2 \pm 3.2) \cdot 10^{-18}$ & $(5.4 \pm 2.8) \cdot 10^{-18}$ \\
& $100^{a}$ & & & $(1.2 \pm 0.7) \cdot 10^{-18}$ \\
PLD-noPd & 7 & & & $(9.5 \pm 4.9) \cdot 10^{-19}$ \\
PLD-Pd & 10 & & & $(2.8 \pm 1.5) \cdot 10^{-21}$ \\
\hline
\end{tabular}

Figure B.5 shows the comparison of the evaluated hydride volume fraction by different methods. The black line shows where data points should be in the case that all methods yield the same results. Mainly the volume $\phi_{M g H 2}^{R}$ was evaluated by the resistance measure as described in chapter 3.3.2. Figure B.5 a) compares these results with the volume fraction $\phi_{M g H 2}^{c}$ estimated from the hydrogen concentration $c_{H}$ taken up by the sample. The hydride volume fraction can be estimated as $c_{H} / 2$, as the hydride is assumed to grow as a layer parallel to the palladium/magnesium and magnesium/silicon interface. The comparison shows that $\phi_{M g H 2}^{c}$ underestimates 
a) Comparison $\phi_{M g H 2}^{R}$ and $\phi_{M g H 2}^{c}$

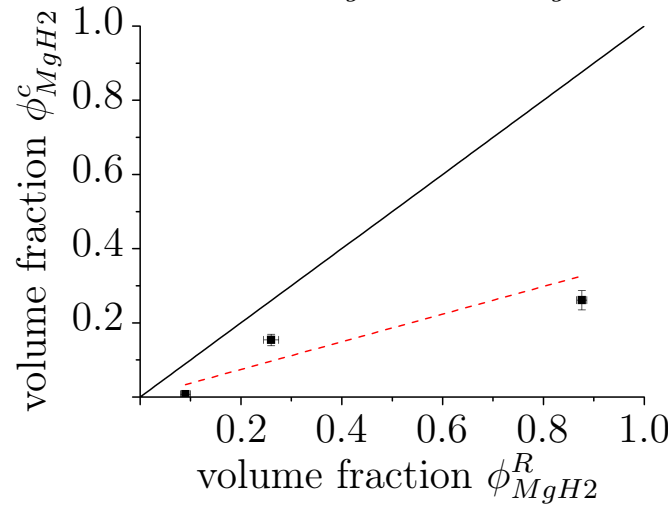

b) Comparison $t_{90 \% M g H 2}^{R}$ and $t_{90 \% M g H 2}^{X}$

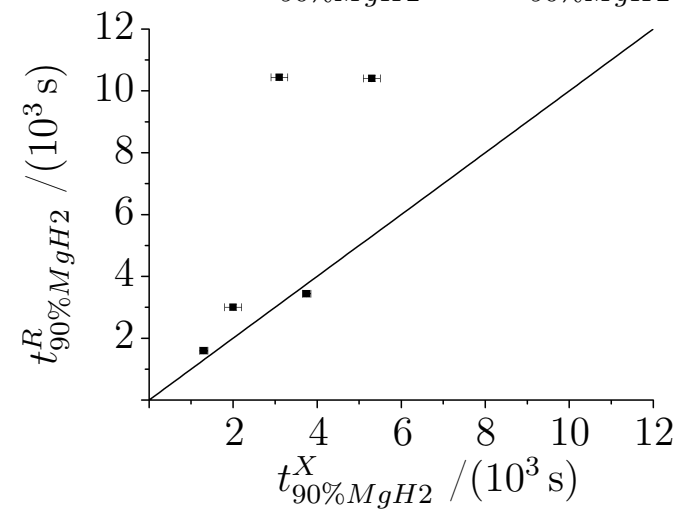

Figure B.5.: Comparison of the evaluated volume fraction of the magnesiumdihydride $\phi_{\mathrm{MgH2} 2}$ by different methods. Figure a) shows the volume fraction at a given time calculated by the resistance measurement $\phi_{M g H 2}^{R}$ compared with the volume fraction estimated from the hydrogen concentration in the sample $\phi_{\mathrm{MgH} 2}^{\mathrm{c}}$. Figure b) compares the time needed to reach $90 \% \mathrm{MgH}_{2}$, on the one side, calculated from the resistance data $t_{90 \% \mathrm{MgH} 2}^{R}$ and measured by the decrease of the $\mathrm{Mg}$ XRD peak $t_{90 \% M g H 2}^{R}$.

the hydride volume fraction compared with $\phi_{M g H 2}^{R}$. From chapter 3.3 .2 it is known that $\phi_{M g H 2}^{R}$ already underestimates the true volume fraction itself. Therefore, $\phi_{M g H 2}^{c}$ needs to be treated with care. Figure B.5 b) compares the time $t_{90 \% \mathrm{MgH} 2}$ necessary to reach $90 \%$ of the overall hydride volume fraction. The y-axis gives the time evaluated by resistance measurements $\left(t_{90 \% M g H 2}^{R}\right)$. The $\mathrm{x}$-axis gives the time evaluated by the decrease of the XRD magnesium (200) peak $\left(t_{90 \% M g H 2}^{X}\right)$. This result is not completely unambiguous. Three of five samples show the same results independent of the method. For two samples the resistance overestimated the time needed to hydride the thin film. However, this is to be expected, as the applied three-layer model underestimates the true hydride volume fraction at a given time. Therefore, the film will form hydride faster than the three-layer model predicts. This effect becomes larger for longer times, corresponding to figure B.5 b). However, overall the three-layer model seems to describe the hydride evolution well. 


\section{Bibliography}

[1] U.S. Department of Energy (DOE): The Department of Energy Hydrogen and Fuel Cells Program Plan (2011). URL https://www.hydrogen.energy. gov/pdfs/program_plan2011.pdf

[2] Conti, J., Holtberg, P., Diefenderfer, J., LaRose, A., Turnure, J. T., Westfall, L.: International Energy Outlook 2016 With Projections to 2040. Technical report, USDOE Energy Information Administration (EIA), Washington, DC (United States). Office of Energy Analysis (2016)

[3] Bundesminesterium für Wirtschaft und Energie (BMWi): Energieeffizienz in Zahlen (2017). URL https://www.bmwi.de/Redaktion/DE/ Publikationen/Energie/energieeffizienz-in-zahlen.html

[4] Petit, J. R., et al.: Climate and atmospheric history of the past 420,000 years from the Vostok ice core, Antarctica. In: Nature, 399:6735, pp. 429-436 (1999)

[5] Mann, M. E., Bradley, R. S., Hughes, M. K.: Northern hemisphere temperatures during the past millennium: Inferences, uncertainties, and limitations. In: Geophysical Research Letters, 26:6, pp. 759-762 (1999)

[6] IPCC Secretariat, World Meteorological Organization: International panel on climate change (IPCC) (2018). URL http://www.ipcc.ch/. Online; accessed 19.04.2018

[7] Laden, F., Neas, L. M., Dockery, D. W., Schwartz, J.: Association of Fine Particulate Matter from Different Sources with Daily Mortality in Six U.S. Cities. In: Environmental Health Perspectives, 108:10, pp. 941-947 (2000)

[8] Grantz, D., Garner, J., Johnson, D.: Ecological effects of particulate matter. In: Environment International, 29:2-3, pp. 213-239 (2003)

[9] Pope, C. A., Ezzati, M., Dockery, D. W.: Fine-Particulate Air Pollu- 
tion and Life Expectancy in the United States. In: New England Journal of Medicine, 360:4, pp. 376-386 (2009)

[10] Adolf, J., Balzer, C. H., Louis, J., Schabla, U., Fischedick, M., Arnold, K., Pastowski, A., Schüwer, D.: Shell Hydrogen Study (2017). URL https://www.shell.de/medien/shell-publikationen/ shell-hydrogen-study.html

[11] Amsterdam Roundtables Foundation, McKinsey \& Company: Electric vehicles in Europe: Gearing up for a new phase? (2014). URL https://www.mckinsey.com/global-themes/europe/ electric-vehicles-in-europe-gearing-up-for-a-new-phase

[12] Offer, G., Howey, D., Contestabile, M., Clague, R., Brandon, N.: Comparative analysis of battery electric, hydrogen fuel cell and hybrid vehicles in a future sustainable road transport system. In: Energy policy, 38:1, pp. 24-29 (2010)

[13] Züttel, A.: Materials for hydrogen storage. In: Materials today, 6:9, pp. 24-33 (2003)

[14] Züttel, A., Remhof, A., Borgschulte, A., Friedrichs, O.: Hydrogen: the future energy carrier. In: Philosophical Transactions of the Royal Society of London A: Mathematical, Physical and Engineering Sciences, 368:1923, pp. 3329-3342 (2010)

[15] OECD, IEA: Hydrogen production and storage: R\&D priorities and gaps (2006). URL https://webstore.iea.org/ hydrogen-production-and-storage

[16] Lipman, T.: An Overview of Hydrogen Production and Storage Systems with Renewable Hydrogen Case Studies (2011). URL https://cesa.org/assets/2011-Files/Hydrogen-and-Fuel-Cells/ CESA-Lipman-H2-prod-storage-050311.pdf

[17] Jensen, J., Vestbø, A., Li, Q., Bjerrum, N.: The energy efficiency of onboard hydrogen storage. In: Journal of Alloys and Compounds, 446, pp. 723-728 (2007)

[18] Fuel Cell Technologies Office: Fuel Cell Technologies Office Multi-Year Research, Development, and Demonstration (2012). URL https://www.energy.gov/eere/fuelcells/downloads/ fuel-cell-technologies-office-multi-year-research-development-and-22 
[19] Dornheim, M., Doppiu, S., Barkhordarian, G., Boesenberg, U., Klassen, T., Gutfleisch, O., Bormann, R.: Hydrogen storage in magnesium-based hydrides and hydride composites. In: Scripta Materialia, 56:10, pp. 841-846 (2007)

[20] Yao, X., Lu, G.: Magnesium-based materials for hydrogen storage: Recent advances and future perspectives. In: Science Bulletin, 53:16, pp. 2421-2431 (2008)

[21] Crivello, J.-C., et al.: Review of magnesium hydride-based materials: development and optimisation. In: Applied Physics A, 122:2 (2016)

[22] InfoMine Inc. : Historical Magnesium Prices and Price Chart (2018). URL http://www.infomine.com/investment/metal-prices/ magnesium/all/. Online; accessed 19.04.2018

[23] Selvam, P., Viswanathan, B., Swamy, C., Srinivasan, V.: Magnesium and magnesium alloy hydrides. In: International Journal of Hydrogen Energy, 11:3, pp. 169-192 (1986)

[24] San-Martin, A., Manchester, F. D.: The H-Mg (Hydrogen-Magnesium) system. In: Journal of Phase Equilibria, 8:5, pp. 431-437 (1987)

[25] Jain, I., Lal, C., Jain, A.: Hydrogen storage in Mg: A most promising material. In: International Journal of Hydrogen Energy, 35:10, pp. 5133-5144 (2010)

[26] Pivak, Y., Schreuders, H., Dam, B.: Thermodynamic Properties, Hysteresis Behavior and Stress-Strain Analysis of MgH2 Thin Films, Studied over a Wide Temperature Range. In: Crystals, 2:2, pp. 710-729 (2012)

[27] Fukai, Y.: The Metal-Hydrogen System, volume 21. Springer Berlin Heidelberg (2006)

[28] Kirchheim, R., Pundt, A.: Hydrogen in Metals. In: Laughlin, D. E., Hono, K. (Hrsg.), Physical Metallurgy, chapter 25, pp. 2597 - 2705. Elsevier, Oxford, fifth edition (2014)

[29] Mushnikov, N. V., Ermakov, A. E., Uimin, M. A., Gaviko, V. S., Terent'ev, P. B., Skripov, A. V., Tankeev, A. P., Soloninin, A. V., Buzlukov, A. L.: Kinetics of interaction of Mg-based mechanically activated alloys with hydrogen. In: The Physics of Metals and Metallography, 102:4, pp. 421-431 (2006) 
[30] Krozer, A., Kasemo, B.: Hydrogen uptake by Pd-coated Mg: absorptiondecomposition isotherms and uptake kinetics. In: Journal of the Less Common Metals, 160:2, pp. 323-342 (1990)

[31] Gérard, N., Ono, S.: Hydride formation and decomposition kinetics. In: Schlapbach, L. (Hrsg.), Hydrogen in Intermetallic Compunds II. Topics in Applied Physics, chapter 4, pp. 165-195. Springer Berlin Heidelberg (1992)

[32] Zaluska, A., Zaluski, L., Ström-Olsen, J.: Nanocrystalline magnesium for hydrogen storage. In: Journal of Alloys and Compounds, 288:1-2, pp. 217-225 (1999)

[33] Andreasen, A., Vegge, T., Pedersen, A. S.: Compensation effect in the hydrogenation/dehydrogenation kinetics of metal hydrides. In: The Journal of Physical Chemistry B, 109:8, pp. 3340-3344 (2005)

[34] Barkhordarian, G., Klassen, T., Bormann, R.: Effect of Nb2O5 content on hydrogen reaction kinetics of $\mathrm{Mg}$. In: Journal of Alloys and Compounds, 364:1-2, pp. 242-246 (2004)

[35] Oelerich, W., Klassen, T., Bormann, R.: Metal oxides as catalysts for improved hydrogen sorption in nanocrystalline Mg-based materials. In: Journal of Alloys and Compounds, 315:1, pp. 237-242 (2001)

[36] Krozer, A., Kasemo, B.: Equilibrium hydrogen uptake and associated kinetics for the Mg-H2 system at low pressures. In: Journal of Physics: Condensed Matter, 1:8, pp. 1533 (1989)

[37] Singh, S., Eijt, S., Zandbergen, M., Legerstee, W., Svetchnikov, V.: Nanoscale structure and the hydrogenation of Pd-capped magnesium thin films prepared by plasma sputter and pulsed laser deposition. In: Journal of Alloys and Compounds, 441:1-2, pp. 344-351 (2007)

[38] Callini, E., Pasquini, L., Piscopiello, E., Montone, A., Antisari, M. V., Bonetti, E.: Hydrogen sorption in Pd-decorated $M g-M g O$ core-shell nanoparticles. In: Applied Physics Letters, 94:22, pp. 221905 (2009)

[39] Vigeholm, B., Kjøller, J., Larsen, B.: Magnesium for hydrogen storage. In: Journal of the Less Common Metals, 74:2, pp. 341-350 (1980)

[40] Chen, C. P., Liu, B. H., Li, Z. P., Wu, J., Wang, Q. D.: The Activation Mechanism of Mg-Based Hydrogen Storage Alloys. In: Zeitschrift für Physikalische Chemie, 181:Part_1_2, pp. 259-267 (1993) 
[41] Luz, Z., Genossar, J., Rudman, P.: Identification of the diffusing atom in MgH2. In: Journal of the Less Common Metals, 73:1, pp. 113-118 (1980)

[42] Barkhordarian, G., Klassen, T., Bormann, R.: Kinetic investigation of the effect of milling time on the hydrogen sorption reaction of magnesium catalyzed with different Nb2O5 contents. In: Journal of Alloys and Compounds, 407:1-2, pp. 249-255 (2006)

[43] Krozer, A., Kasemo, B.: Unusual kinetics due to interface hydride formation in the hydriding of $\mathrm{Pd} / \mathrm{Mg}$ sandwich layers. In: Journal of Vacuum Science \& Technology A: Vacuum, Surfaces, and Films, 5:4, pp. 1003-1005 (1987)

[44] Uchida, H., Wagner, S., Hamm, M., Kürschner, J., Kirchheim, R., Hjörvarsson, B., Pundt, A.: Absorption kinetics and hydride formation in magnesium films: Effect of driving force revisited. In: Acta Materialia, 85, pp. 279-289 (2015)

[45] Hao, S., Sholl, D. S.: Hydrogen diffusion in MgH[sub 2] and NaMgH[sub 3] via concerted motions of charged defects. In: Applied Physics Letters, 93:25, pp. 251901 (2008)

[46] Vigeholm, B., Jensen, K., Larsen, B., Pedersen, A.: Elements of hydride formation mechanisms in nearly spherical magnesium powder particles. In: Journal of the Less Common Metals, 131:1-2, pp. 133-141 (1987)

[47] Reule, H., Hirscher, M., Weißhardt, A., Kronmüller, H.: Hydrogen desorption properties of mechanically alloyed MgH2 composite materials. In: Journal of Alloys and Compounds, 305:1-2, pp. 246-252 (2000)

[48] Lang, J., Huot, J.: A new approach to the processing of metal hydrides. In: Journal of Alloys and Compounds, 509:3, pp. L18-L22 (2011)

[49] Skripnyuk, V., Rabkin, E., Estrin, Y., Lapovok, R.: Improving hydrogen storage properties of magnesium based alloys by equal channel angular pressing. In: International Journal of Hydrogen Energy, 34:15, pp. 6320-6324 (2009)

[50] Krystian, M., Zehetbauer, M., Kropik, H., Mingler, B., Krexner, G.: Hydrogen storage properties of bulk nanostructured ZK60 Mg alloy processed by Equal Channel Angular Pressing. In: Journal of Alloys and Compounds, 509, pp. S449-S455 (2011)

[51] Kaur, I., Mishin, Y., Gust, W., et al.: Fundamentals of grain and interphase boundary diffusion. John Wiley (1995) 
[52] McNabb, A., Foster, P.: A new analysis of the diffusion of hydrogen in iron and ferritic steels. In: Transactions of the Metallurgical Society of AIME, 227:3, pp. 618-627 (1963)

[53] Oriani, R. A.: The diffusion and trapping of hydrogen in steel. In: Acta metallurgica, 18:1, pp. 147-157 (1970)

[54] Leblond, J., Dubois, D.: A general mathematical description of hydrogen diffusion in steels - II. Numerical study of permeation and determination of trapping parameters. In: Acta metallurgica, 31:10, pp. 1471-1478 (1983)

[55] Krom, A. H., Bakker, A.: Hydrogen trapping models in steel. In: Metallurgical and materials transactions B, 31:6, pp. 1475-1482 (2000)

[56] Oudriss, A., Creus, J., Bouhattate, J., Conforto, E., Berziou, C., Savall, C., Feaugas, X.: Grain size and grain-boundary effects on diffusion and trapping of hydrogen in pure nickel. In: Acta Materialia, 60:19, pp. 6814$6828(2012)$

[57] Oudriss, A., Creus, J., Bouhattate, J., Savall, C., Peraudeau, B., Feaugas, X.: The diffusion and trapping of hydrogen along the grain boundaries in polycrystalline nickel. In: Scripta Materialia, 66:1, pp. 37-40 (2012)

[58] Fisher, J. C.: Calculation of Diffusion Penetration Curves for Surface and Grain Boundary Diffusion. In: Journal of Applied Physics, 22:1, pp. 74 (1951)

[59] Whipple, R.: CXXXVIII. Concentration contours in grain boundary diffusion. In: The London, Edinburgh, and Dublin Philosophical Magazine and Journal of Science, 45:371, pp. 1225-1236 (1954)

[60] Levine, H. S., MacCallum, C. J.: Grain Boundary and Lattice Diffusion in Polycrystalline Bodies. In: Journal of Applied Physics, 31:3, pp. 595 (1960)

[61] LeClaire, A. D.: The analysis of grain boundary diffusion measurements. In: British Journal of Applied Physics, 14:6, pp. 351 (1963)

[62] Suzuoka, T.: Lattice and Grain Boundary Diffusion in Polycrystals. In: Transactions of the Japan Institute of Metals, 2:1, pp. 25-32 (1961)

[63] Suzuoka, T.: Exact Solutions of Two Ideal Cases in Grain Boundary Diffusion Problem and the Application to Sectioning Method. In: Journal of the Physical Society of Japan, 19:6, pp. 839-851 (1964)

[64] Harrison, L. G.: Influence of dislocations on diffusion kinetics in solids with 
particular reference to the alkali halides. In: Transactions of the Faraday Society, 57, pp. 1191 (1961)

[65] Kaur, I., Gust, W., Kozma, L.: Handbook of grain and interphase boundary diffusion data. Bd. 1. Ziegler Press (1989)

[66] Mishin, Y., Herzig, C.: Grain boundary diffusion: recent progress and future research. In: Materials Science and Engineering: A, 260:1-2, pp. 55-71 (1999)

[67] Mishin, Y. M.: 50 Years of Grain Boundary Diffusion: What Do We Know about It Today? In: Defect and Diffusion Forum, volume 194-199, pp. 11131126. Trans Tech Publ, Trans Tech Publications (2001)

[68] Divinski, S. V., Edelhoff, H., Prokofjev, S.: Diffusion and segregation of silver in copper $\Sigma 5$ (310) grain boundary. In: Physical Review B, 85:14, pp. $144104(2012)$

[69] Nayeb-Hashemi, A. A., Clark, J. B., Swartzendruber, L. J.: The FeMg (Iron-Magnesium) system. In: Bulletin of Alloy Phase Diagrams, 6:3, pp. 235-238 (1985)

[70] Okamoto, H.: H-Mg (Hydrogen-Magnesium). In: Journal of Phase Equilibria, 22:5, pp. 598-599 (2001)

[71] Ham, B., et al.: Hydrogen sorption in orthorhombic Mg hydride at ultralow temperature. In: International Journal of Hydrogen Energy, 38:20, pp. 8328-8341 (2013)

[72] Moser, D., Baldissin, G., Bull, D. J., Riley, D. J., Morrison, I., Ross, D. K., Oates, W. A., Noréus, D.: The pressure-temperature phase diagram of $\mathrm{MgH} 2 \mathrm{a}$ and isotopic substitution. In: Journal of Physics: Condensed Matter, 23:30, pp. 305403 (2011)

[73] Pundt, A., Kirchheim, R.: HYDROGEN IN METALS: Microstructural Aspects. In: Annual Review of Materials Research, 36:1, pp. 555-608 (2006)

[74] Litiagina, L., Diuzheva, T., Kabalkina, S., Dymova, T., Losev, V.: The polymorphism of MgH2 at pressures up to 12 GPa. In: Geokhimiia, 1, pp. 118-120 (1985)

[75] Ravindran, P., Vajeeston, P., Fjellvåg, H., Kjekshus, A.: Chemicalbonding and high-pressure studies on hydrogen-storage materials. In: Computational Materials Science, 30:3-4, pp. 349-357 (2004) 
[76] Uchida, H. T.: Hydrogen absorption property of nanocrystalline-magnesium films. Ph.D. thesis, Dissertation, Göttingen, Georg-August Universität, 2015 (2015). URL http://hdl.handle.net/11858/00-1735-0000-002B-7C91-C

[77] Stampfer, J. F., Holley, C. E., Suttle, J. F.: The Magnesium-Hydrogen System1-3. In: Journal of the American Chemical Society, 82:14, pp. 35043508 (1960)

[78] Borgschulte, A., Bösenberg, U., Barkhordarian, G., Dornheim, M., Bormann, R.: Enhanced hydrogen sorption kinetics of magnesium by destabilized MgH 2- $\delta$. In: Catalysis Today, 120:3, pp. 262-269 (2007)

[79] Schimmel, H., Kearley, G., Huot, J., Mulder, F.: Hydrogen diffusion in magnesium metal ( phase) studied by ab initio computer simulations. In: Journal of Alloys and Compounds, 404-406, pp. 235-237 (2005)

[80] Rudman, P.: Thermodynamics of pressure plateaus in metal-hydrogen systems. In: International Journal of Hydrogen Energy, 3:4, pp. 431-447 (1978)

[81] Vigeholm, B., Kjøller, J., Larsen, B., Pedersen, A.: Formation and decomposition of magnesium hydride. In: Journal of the Less Common Metals, 89:1, pp. 135-144 (1983)

[82] Klose, W., Stuke, V.: Investigation of the thermodynamic equilibrium in the hydrogen-magnesium-magnesium hydride system. In: International Journal of Hydrogen Energy, 20:4, pp. 309-316 (1995)

[83] Bogdanović, B., Bohmhammel, K., Christ, B., Reiser, A., Schlichte, K., Vehlen, R., Wolf, U.: Thermodynamic investigation of the magnesium-hydrogen system. In: Journal of Alloys and Compounds, 282:1-2, pp. 84-92 (1999)

[84] Wagner, S., Pundt, A.: Mechanical stress impact on thin Pd1-xFex film thermodynamic properties. In: Applied Physics Letters, 92:5, pp. 051914 (2008)

[85] Wagner, S., Pundt, A.: Quasi-thermodynamic model on hydride formation in palladium-hydrogen thin films: Impact of elastic and microstructural constraints. In: International Journal of Hydrogen Energy, 41:4, pp. 2727-2738 (2016)

[86] Burlaka, V., Wagner, S., Hamm, M., Pundt, A.: Suppression of Phase Transformation in $\mathrm{Nb}-\mathrm{H}$ Thin Films below Switchover Thickness. In: Nano Letters, 16:10, pp. 6207-6212 (2016) 
[87] Laudahn, U., Fähler, S., Krebs, H. U., Pundt, A., Bicker, M., v. Hülsen, U., Geyer, U., Kirchheim, R.: Determination of elastic constants in thin films using hydrogen loading. In: Applied Physics Letters, 74:5, pp. 647-649 (1999)

[88] Hamm, M., Burlaka, V., Wagner, S., Pundt, A.: Achieving reversibility of ultra-high mechanical stress by hydrogen loading of thin films. In: Applied Physics Letters, 106:24, pp. 243108 (2015)

[89] Landau, L. D., Lifshitz, E. M.: Theory of Elasticity 7. Elsevier LTD, Oxford (2004)

[90] Sander, D.: The correlation between mechanical stress and magnetic anisotropy in ultrathin films. In: Reports on Progress in Physics, 62:5, pp. 809-858 (1999)

[91] Huntington, H.: The Elastic Constants of Crystals. In: Solid State Physics, volume 7, pp. 213-351. Elsevier (1958)

[92] Zarshenas, M., Ahmed, R., Kanoun, M. B., ul Haq, B., Isa, A. R. M., Goumri-Said, S.: First principle investigations of the physical properties of hydrogen-rich MgH2. In: Physica Scripta, 88:6, pp. 065704 (2013)

[93] Bastide, J.-P., Bonnetot, B., Létoffé, J.-M., Claudy, P.: Polymorphisme de l'hydrure de magnesium sous haute pression. In: Materials Research Bulletin, 15:12, pp. 1779-1787 (1980)

[94] Bortz, M., Bertheville, B., Böttger, G., Yvon, K.: Structure of the high pressure phase $\gamma-\mathrm{MgH}_{2}$ by neutron powder diffraction. In: Journal of Alloys and Compounds, 287:1-2, pp. L4-L6 (1999)

[95] Varin, R. A., Czujko, T., Wronski, Z.: Particle size, grain size and $\gamma-M g H$ 2 effects on the desorption properties of nanocrystalline commercial magnesium hydride processed by controlled mechanical milling. In: Nanotechnology, $17: 15$, pp. 3856-3865 (2006)

[96] Leiva, D. R., Jorge, A. M., Ishikawa, T. T., Huot, J., Fruchart, D., Miraglia, S., Kiminami, C. S., Botta, W. J.: Nanoscale Grain Refinement and H-Sorption Properties of MgH2 Processed by High-Pressure Torsion and Other Mechanical Routes. In: Advanced Engineering Materials, 12:8, pp. 786-792 (2010)

[97] Vajeeston, P., Ravindran, P., Hauback, B. C., Fjellvåg, H., Kjekshus, 
A., Furuseth, S., Hanfland, M.: Structural stability and pressure-induced phase transitions inMgH2. In: Physical Review B, 73:22, pp. 224102 (2006)

[98] Cui, S., Feng, W., Hu, H., Feng, Z., Wang, Y.: Structural phase transitions in MgH2 under high pressure. In: Solid State Communications, 148:9, pp. 403-405 (2008)

[99] Vajeeston, P., Ravindran, P., Kjekshus, A., Fjellvåg, H.: PressureInduced Structural Transitions inMgH2. In: Physical Review Letters, 89:17, pp. 175506 (2002)

[100] Avedesian, M. M.: Magnesium and Magnesium Alloys (Asm Specialty Handbook) (Asm Specialty Handbook). ASM International (1999)

[101] Westerwaal, R., Broedersz, C., Gremaud, R., Slaman, M., Borgschulte, A., Lohstroh, W., Tschersich, K., Fleischhauer, H., Dam, B., Griessen, R.: Study of the hydride forming process of in-situ grown MgH2 thin films by activated reactive evaporation. In: Thin Solid Films, 516:12, pp. 4351-4359 (2008)

[102] He, Z. X., Pong, W.: X-ray photoelectron spectra of MgH2. In: Physica Scripta, 41:6, pp. 930-932 (1990)

[103] Paik, B., Walton, A., Mann, V., Book, D., Jones, I. P., Harris, I. R.: Electron energy-loss spectroscopy study of MgH2 in the plasmon energy range. In: Applied Physics Letters, 100:19, pp. 193902 (2012)

[104] Giebels, I. A. M. E., Isidorsson, J., Griessen, R.: Highly absorbing black Mg and rare-earth-Mg switchable mirrors. In: Physical Review B, 69:20 (2004)

[105] Mayadas, A. F., Shatzkes, M.: Electrical-Resistivity Model for Polycrystalline Films: the Case of Arbitrary Reflection at External Surfaces. In: Physical Review B, 1:4, pp. 1382-1389 (1970)

[106] Demetry, C.: Grain size-dependent electrical properties of rutile (TiO2). In: Solid State Ionics, 118:3-4, pp. 271-279 (1999)

[107] Sieverts, A.: Absorption of gases by metals. In: Zeitschrift für Metallkunde, 21, pp. 37-46 (1929)

[108] Chen, C., Splinter, S., Do, T., McIntyre, N.: Measurement of oxide film growth on $M g$ and Al surfaces over extended periods using XPS. In: Surface Science, 382:1-3, pp. L652-L657 (1997) 
[109] Fournier, V., Marcus, P., Olefjord, I.: Oxidation of magnesium. In: Surface and Interface Analysis, 34:1, pp. 494-497 (2002)

[110] Barkhordarian, G., Klassen, T., Bormann, R.: Fast hydrogen sorption kinetics of nanocrystalline Mg using Nb2O5 as catalyst. In: Scripta Materialia, 49:3, pp. 213-217 (2003)

[111] Vincent, S., Lang, J., Huot, J.: Addition of catalysts to magnesium hydride by means of cold rolling. In: Journal of Alloys and Compounds, 512:1, pp. 290-295 (2012)

[112] Pozzo, M., Alfè, D.: Hydrogen dissociation and diffusion on transition metal (=Ti, $\mathrm{Zr}, \mathrm{V}, \mathrm{Fe}, \mathrm{Ru}, \mathrm{Co}, \mathrm{Rh}, \mathrm{Ni}, \mathrm{Pd}, \mathrm{Cu}, \mathrm{Ag}$ )-doped Mg(0001) surfaces. In: International Journal of Hydrogen Energy, 34:4, pp. 1922-1930 (2009)

[113] Welter, J.-M., Rudman, P.: Iron catalyzed hydriding of magnesium. In: Scripta Metallurgica, 16:3, pp. 285-286 (1982)

[114] Holtz, R. L., Imam, M. A.: Hydrogen storage characteristics of ball-milled magnesium-nickel and magnesium-iron alloys. In: Journal of Materials Science, 34:11, pp. 2655-2663 (1999)

[115] Baum, L., Meyer, M., Mendoza-Zélis, L.: Hydrogen storage properties of the $\mathrm{Mg} / \mathrm{Fe}$ system. In: Physica B: Condensed Matter, 389:1, pp. 189-192 (2007)

[116] San-Martin, A., Manchester, F. D.: The Fe-H (Iron-Hydrogen) system. In: Bulletin of Alloy Phase Diagrams, 11:2, pp. 173-184 (1990)

[117] Didisheim, J. J., Zolliker, P., Yvon, K., Fischer, P., Schefer, J., Gubelmann, M., Williams, A. F.: Dimagnesium iron(II) hydride, Mg2FeH6, containing octahedral FeH64- anions. In: Inorganic Chemistry, 23:13, pp. 1953-1957 (1984)

[118] Bogdanović, B., Reiser, A., Schlichte, K., Spliethoff, B., Tesche, B.: Thermodynamics and dynamics of the $\mathrm{Mg}-\mathrm{Fe}-\mathrm{H}$ system and its potential for thermochemical thermal energy storage. In: Journal of Alloys and Compounds, 345:1-2, pp. 77-89 (2002)

[119] Lima, G., Peres, M., Garroni, S., Baró, M., Surinyach, S., Kiminami, C., Ishikawa, T., Botta, W., Jorge, A.: Microstructural characterization and hydrogenation study of extruded MgFe alloy. In: Journal of Alloys and Compounds, 504, pp. S299-S301 (2010) 
[120] de Lima, G. F., Garroni, S., Baró, M. D., Suriñach, S., Kiminami, C. S., Botta, W. J., Peres, M. M., Junior, A. M. J.: $2 M g-F e$ alloys processed by hot-extrusion: Influence of processing temperature and the presence of $\mathrm{MgO}$ and $\mathrm{MgH} 2$ on hydrogenation sorption properties. In: Journal of Alloys and Compounds, 509, pp. S460-S463 (2011)

[121] de Lima, G., Garroni, S., Baró, M., Suriñach, S., Kiminami, C., Botta, W., Jorge, A.: Hydrogen storage properties of $2 M g-F e$ mixtures processed by hot extrusion: Influence of the extrusion ratio. In: International Journal of Hydrogen Energy, 37:20, pp. 15196-15203 (2012)

[122] Manchester, F. D., San-Martin, A., Pitre, J. M.: The H-Pd (hydrogenpalladium) System. In: Journal of Phase Equilibria, 15:1, pp. 62-83 (1994)

[123] Makongo, J. P. A., Prots, Y., Burkhardt, U., Niewa, R., Kudla, C., Kreiner, G.: A case study of complex metallic alloy phases: structure and disorder phenomena of $M g-P d$ compounds. In: Philosophical Magazine, 86:3-5, pp. 427-433 (2006)

[124] Slack, J. L., Locke, J. C., Song, S.-W., Ona, J., Richardson, T. J.: Metal hydride switchable mirrors: Factors influencing dynamic range and stability. In: Solar Energy Materials and Solar Cells, 90:4, pp. 485-490 (2006)

[125] Eijt, S. W. H., Kind, R., Singh, S., Schut, H., Legerstee, W. J., Hendrikx, R. W. A., Svetchnikov, V. L., Westerwaal, R. J., Dam, B.: Positron depth profiling of the structural and electronic structure transformations of hydrogenated Mg-based thin films. In: Journal of Applied Physics, 105:4, pp. 043514 (2009)

[126] Nayeb-Hashemi, A. A., Clark, J. B.: The Mg-Pd (magnesium-palladium) system. In: Bulletin of Alloy Phase Diagrams, 6:2, pp. 164-167 (1985)

[127] Zhang, W., Luo, S., Flanagan, T. B.: Hydrogen solution in homogeneous Pd-Fe alloys. In: Journal of Alloys and Compounds, 293-295, pp. 1-6 (1999)

[128] Klose, P., Hamm, M., Roddatis, V., Pundt, A.: Influence of steel on the mechanical stress development during hydrogen-loading of ultrathin Nb-films. In: International Journal of Hydrogen Energy, 42:35, pp. 22583-22588 (2017)

[129] Fuggle, J., Watson, L., Fabian, D., Affrossman, S.: X-ray photoelectron studies of the reaction of clean metals $(\mathrm{Mg}, \mathrm{Al}, \mathrm{Cr}, \mathrm{Mn})$ with oxygen and water vapour. In: Surface Science, 49:1, pp. 61-76 (1975)

[130] Splinter, S., McIntyre, N., Lennard, W., Griffiths, K., Palumbo, G.: 
An AES and XPS study of the initial oxidation of polycrystalline magnesium with water vapour at room temperature. In: Surface Science, 292:1-2, pp. 130144 (1993)

[131] Feliu Jr, S., Galván, J., Pardo, A., Merino, M., Arrabal, R.: Native air-formed oxide film and its effect on magnesium alloys corrosion. In: The Open Corrosion Journal, 3, pp. 80-91 (2010)

[132] Kuroda, Y., Yasugi, E., Aoi, H., Miura, K., Morimoto, T.: Interaction of water with the surface of magnesium oxide. In: Journal of the Chemical Society, Faraday Transactions 1: Physical Chemistry in Condensed Phases, 84:7, pp. 2421-2430 (1988)

[133] Song, S.-H., Wang, X., Xiao, P.: Effect of microstructural features on the electrical properties of TiO 2. In: Materials Science and Engineering: B, 94:1, pp. 40-47 (2002)

[134] Gonzalez, R., Chen, Y., Tsang, K.: Diffusion of deuterium and hydrogen in doped and undoped $M g O$ crystals. In: Physical review B, 26:8, pp. 4637 (1982)

[135] Hjort, P., Krozer, A., Kasemo, B.: Hydrogen sorption kinetics in partly oxidized Mg films. In: Journal of Alloys and Compounds, 237:1-2, pp. 74-80 (1996)

[136] Evard, E., Gabis, I., Yartys, V.: Kinetics of hydrogen evolution from MgH2: Experimental studies, mechanism and modelling. In: International Journal of Hydrogen Energy, 35:17, pp. 9060-9069 (2010)

[137] Tanniru, M., Tien, H.-Y., Ebrahimi, F.: Study of the dehydrogenation behavior of magnesium hydride. In: Scripta Materialia, 63:1, pp. 58-60 (2010)

[138] Čermák, J., Král, L.: Hydrogen diffusion in $M g-H$ and $M g-N i-H$ alloys. In: Acta Materialia, 56:12, pp. 2677-2686 (2008)

[139] Spatz, P., Aebischer, H. A., Krozer, A., Schlapbach, L.: The Diffusion of $\mathrm{H}$ in $\mathrm{Mg}$ and the Nucleation and Growth of $M g H 2$ in Thin Films. In: Zeitschrift für Physikalische Chemie, 181:Part_1_2, pp. 393-397 (1993)

[140] Qu, J., Sun, B., Yang, R., Zhao, W., Wang, Y., Li, X.: Hydrogen absorption kinetics of $\mathrm{Mg}$ thin films under mild conditions. In: Scripta Materialia, 62:5, pp. 317-320 (2010)

[141] Vegge, T.: Locating the rate-limiting step for the interaction of hydrogen 
with $\mathrm{Mg}(0001)$ using density-functional theory calculations and rate theory. In: Physical Review B, 70:3 (2004)

[142] Nishimura, C., Komaki, M., Amano, M.: Hydrogen permeation through magnesium. In: Journal of Alloys and Compounds, 293-295, pp. 329-333 (1999)

[143] Renner, J., Grabke, H.: Bestimmung von Diffusionskoeffizienten bei der Hydrierung von Legierungen. In: Zeitschrift für Metallkunde, 69, pp. 639-642 (1978)

[144] Stander, C. M.: Kinetics of Formation of Magnesium Hydride from Magnesium and Hydrogen. In: Zeitschrift für Physikalische Chemie, 104:4-6, pp. 229-238 (1977)

[145] Simensen, C. J.: Gas-chromatographic determination of hydrogen in magnesium by means of the capsule method. In: Fresenius Zeitschrift für Analytische Chemie, 302:5, pp. 398-401 (1980)

[146] Bobet, J., Aymonier, C., Mesguich, D., Cansell, F., Asano, K., Akiba, E.: Particle decoration in super critical fluid to improve the hydrogen sorption cyclability of magnesium. In: Journal of Alloys and Compounds, 429:1-2, pp. 250-254 (2007)

[147] Töpler, J., Buchner, H., Säufferer, H., Knorr, K., Prandl, W.: Measurements of the diffusion of hydrogen atoms in magnesium and Mg2Ni by neutron scattering. In: Journal of the Less Common Metals, 88:2, pp. 397-404 (1982)

[148] Corey, R. L., et al.: Hydrogen Motion in Magnesium Hydride by NMR. In: The Journal of Physical Chemistry C, 112:49, pp. 19784-19790 (2008)

[149] Yao, X., Zhu, Z., Cheng, H., Lu, G.: Hydrogen diffusion and effect of grain size on hydrogenation kinetics in magnesium hydrides. In: Journal of Materials Research, 23:02, pp. 336-340 (2008)

[150] Stioui, M., Grayevsky, A., Resnik, A., Shaltiel, D., Kaplan, N.: Macroscopic and microscopic kinetics of hydrogen in magnesium-rich compounds. In: Journal of the Less Common Metals, 123:1-2, pp. 9-24 (1986)

[151] Mintz, M., Gavra, Z., Hadari, Z.: Kinetic study of the reaction between hydrogen and magnesium, catalyzed by addition of indium. In: Journal of Inorganic and Nuclear Chemistry, 40:5, pp. 765-768 (1978) 
[152] Fick, A.: V. On liquid diffusion. In: Philosophical Magazine Series 4, 10:63, pp. 30-39 (1855)

[153] Porter, D., Easterling, K.: Phase transitions in metals and alloys. Chapman \& Hall, London (1992)

[154] Darken, L. S.: Diffusion, mobility and their interrelation through free energy in binary metallic system. In: Transactions of the American Institute of Mining and Metallurgical Engineers, 175, pp. 184 (1948)

[155] Gryaznov, D., Fleig, J., Maier, J.: An improved procedure for determining grain boundary diffusion coefficients from averaged concentration profiles. In: Journal of Applied Physics, 103:6, pp. 063717 (2008)

[156] Lejcek, P.: Grain Boundary Segregation in Metals, volume 136. Springer Berlin Heidelberg (2010)

[157] Suzuki, A., Mishin, Y.: Atomic mechanisms of grain boundary diffusion: Low versus high temperatures. In: Journal of Materials Science, 40:12, pp. 3155-3161 (2005)

[158] Hoffman, R.: Anisotropy of grain boundary self-diffusion. In: Acta Metallurgica, 4:1, pp. $97-98$ (1956)

[159] Johnson, O., Paek, S.-H., DeFord, J. W.: Diffusion of $H$ and $D$ in TiO2: Suppression of internal fields by isotope exchange. In: Journal of Applied Physics, 46:3, pp. 1026-1033 (1975)

[160] Gottstein, G.: Physical foundations of materials science. Springer Science \& Business Media (2013)

[161] Herbeuval, I., Biscondi, M.: Diffusion du zinc dans les joints de flexion symétriques de l'aluminium. In: Canadian Metallurgical Quarterly, 13:1, pp. 171-175 (1974)

[162] Aleshin, A., Bokshtein, B., Shvindlerman, L.: Diffusion of Zinc Along 100 Tilt Boundaries in Aluminum. In: Soviet Physics-Solid State, 19:12, pp. 2051-2054 (1977)

[163] Li, X., Chou, Y.: High angle grain boundary diffusion of chromium in niobium bicrystals. In: Acta Materialia, 44:9, pp. 3535-3541 (1996)

[164] Ma, Q., Balluffi, R.: Diffusion along [001] tilt boundaries in the Au/Ag 
system-I. Experimental results. In: Acta Metallurgica et Materialia, 41:1, pp. 133-141 (1993)

[165] Ma, Q., Liu, C., Adams, J., Balluffi, R.: Diffusion along [001] tilt boundaries in the $A u / A g$ system-II. Atomistic modeling and interpretation. In: Acta Metallurgica et Materialia, 41:1, pp. 143-151 (1993)

[166] Han, J., Vitek, V., Srolovitz, D. J.: The grain-boundary structural unit model redux. In: Acta Materialia, 133, pp. 186-199 (2017)

[167] Bishop, G., Chalmers, B.: A coincidence-Ledge-Dislocation description of grain boundaries. In: Scripta Metallurgica, 2:2, pp. 133-139 (1968)

[168] Sutton, A. P., Vitek, V.: On the Structure of Tilt Grain Boundaries in Cubic Metals I. Symmetrical Tilt Boundaries. In: Philosophical Transactions of the Royal Society A: Mathematical, Physical and Engineering Sciences, 309:1506, pp. 1-36 (1983)

[169] Sutton, A. P., Vitek, V.: On the Structure of Tilt Grain Boundaries in Cubic Metals II. Asymmetrical Tilt Boundaries. In: Philosophical Transactions of the Royal Society A: Mathematical, Physical and Engineering Sciences, 309:1506, pp. 37-54 (1983)

[170] Ladna, B., Birnbaum, H.: SIMS study of hydrogen at the surface and grain boundaries of nickel bicrystals. In: Acta Metallurgica, 35:10, pp. 2537-2542 (1987)

[171] Birnbaum, H., Ladna, B., Kimura, A.: Hydrogen segregation and diffusion at grain boundaries. In: Le Journal de Physique Colloques, 49:C5, pp. C5-397-C5-401 (1988)

[172] Mishin, Y., Razumovskii, I.: Analysis of an asymmetrical model for boundary diffusion. In: Acta Metallurgica et Materialia, 40:3, pp. 597-606 (1992)

[173] Belova, I., Murch, G.: Diffusion in nanocrystalline materials. In: Journal of Physics and Chemistry of Solids, 64:5, pp. 873-878 (2003)

[174] Mishin, Y., Razumovskii, I.: A model for diffusion along a moving grain boundary. In: Acta Metallurgica et Materialia, 40:4, pp. 839-845 (1992)

[175] Deng, J., Morgan, D., Szlufarska, I.: Kinetic Monte Carlo simulation of the effective diffusivity in grain boundary networks. In: Computational Materials Science, 93, pp. 36-45 (2014) 
[176] Herzig, C., Divinski, S. V.: Grain Boundary Diffusion in Metals: Recent Developments. In: Materials Transactions, 44:1, pp. 14-27 (2003)

[177] Mütschele, T., Kirchheim, R.: Hydrogen as a probe for the average thickness of a grain boundary. In: Scripta Metallurgica, 21:8, pp. 1101-1104 (1987)

[178] Avrami, M.: Kinetics of Phase Change. I General Theory. In: The Journal of Chemical Physics, 7:12, pp. 1103 (1939)

[179] Avrami, M.: Kinetics of Phase Change. II Transformation-Time Relations for Random Distribution of Nuclei. In: The Journal of Chemical Physics, 8:2, pp. $212(1940)$

[180] Avrami, M.: Granulation, Phase Change, and Microstructure Kinetics of Phase Change. III. In: The Journal of Chemical Physics, 9:2, pp. 177 (1941)

[181] Johnson, W., Mehl, R.: Reaction kinetics in processes of reaction and growth. In: Transactions of the Metallurgical Society of AIME, 135, pp. 42-58 (1939)

[182] Kolmogorov, A. N.: On the statistical theory of the crystallization of metals. In: Bull. Acad. Sci. USSR, Math. Ser, 1, pp. 355-359 (1937)

[183] Khawam, A., Flanagan, D. R.: Solid-State Kinetic Models: Basics and Mathematical Fundamentals. In: The Journal of Physical Chemistry B, 110:35, pp. 17315-17328 (2006)

[184] Vyazovkin, S., Burnham, A. K., Criado, J. M., Pérez-Maqueda, L. A., Popescu, C., Sbirrazzuoli, N.: ICTAC Kinetics Committee recommendations for performing kinetic computations on thermal analysis data. In: Thermochimica Acta, 520:1-2, pp. 1-19 (2011)

[185] Pang, Y., Li, Q.: A review on kinetic models and corresponding analysis methods for hydrogen storage materials. In: International Journal of Hydrogen Energy, 41:40, pp. 18072-18087 (2016)

[186] Pérez-Maqueda, L., Criado, J., Málek, J.: Combined kinetic analysis for crystallization kinetics of non-crystalline solids. In: , 320:1-3, pp. 84-91 (2003)

[187] Liu, F., Sommer, F., Bos, C., Mittemeijer, E. J.: Analysis of solid state phase transformation kinetics: models and recipes. In: International Materials Reviews, 52:4, pp. 193-212 (2007) 
[188] Starink, M.: On the meaning of the impingement parameter in kinetic equations for nucleation and growth reactions. In: Journal of Materials Science, 36:18, pp. 4433-4441 (2001)

[189] Mintz, M. H., Zeiri, Y.: Hydriding kinetics of powders. In: Journal of Alloys and Compounds, 216:2, pp. 159-175 (1995)

[190] Jander, W.: Reaktionen im festen Zustande bei höheren Temperaturen. Reaktionsgeschwindigkeiten endotherm verlaufender Umsetzungen. In: Zeitschrift für anorganische und allgemeine Chemie, 163:1, pp. 1-30 (1927)

[191] Ginstling, A., Brounshtein, B.: Concerning the diffusion kinetics of reactions in spherical particles. In: Journal of applied chemistry of the USSR, 23:12, pp. 1327-1338 (1950)

[192] Qu, J., Wang, Y., Xie, L., Zheng, J., Liu, Y., Li, X.: Superior hydrogen absorption and desorption behavior of $M g$ thin films. In: Journal of Power Sources, 186:2, pp. 515-520 (2009)

[193] Krebs, H.-U., et al.: Pulsed Laser Deposition (PLD) - A Versatile Thin Film Technique. In: Advances in Solid State Physics, pp. 505-518. Springer Berlin Heidelberg (2003)

[194] Eason, R.: Pulsed Laser Deposition of Thin Films: Applications-Led Growth of Functional Materials, chapter 16, pp. 363-380. JOHN WILEY \& SONS INC (2006)

[195] Krebs, H., Störmer, M., Fähler, S., Bremert, O., Hamp, M., Pundt, A., Teichler, H., Blum, W., Metzger, T.: Structural properties of laser deposited metallic alloys and multilayers. In: Applied Surface Science, 109-110, pp. 563-569 (1997)

[196] Krebs, H.-U., Bremert, O.: Pulsed laser deposition of thin metallic alloys. In: Applied Physics Letters, 62:19, pp. 2341-2343 (1993)

[197] Dittrich, A., Eberl, C., Schlenkrich, S., Schlenkrich, F., Döring, F., Krebs, H.-U.: Drastic deviations from stoichiometry transfer during pulsed laser deposition. In: Applied Physics A, 122:4, pp. 301 (2016)

[198] Clapeyron, E.: Über die bewegende Kraft der Wärme. In: Annalen der Physik und Chemie, 135:7, pp. 446-467 (1843)

[199] Carman, P. C., Haul, R. A. W.: Measurement of Diffusion Coefficients. In: 
Proceedings of the Royal Society A: Mathematical, Physical and Engineering Sciences, 222:1148, pp. 109-118 (1954)

[200] Carslaw, H. S., Jaeger, J. C.: Conduction of heat in solids. Clarendon Press (1960)

[201] Crank, J.: The mathematics of diffusion. Oxford university press (1979)

[202] Ma, Y. H., Evans, L. B.: Transient diffusion from a well-stirred reservoir to a body of arbitrary shape. In: AIChE Journal, 14:6, pp. 956-961 (1968)

[203] San Marchi, C., Somerday, B., Robinson, S.: Permeability, solubility and diffusivity of hydrogen isotopes in stainless steels at high gas pressures. In: International Journal of Hydrogen Energy, 32:1, pp. 100-116 (2007)

[204] Louthan, M., Derrick, R.: Hydrogen transport in austenitic stainless steel. In: Corrosion Science, 15:6-12, pp. 565-577 (1975)

[205] Caskey, G. R., Sisson, R.: Hydrogen solubility in austenitic stainless steels. In: Scripta Metallurgica, 15:11, pp. 1187-1190 (1981)

[206] Xiukui, S., Jian, X., Yiyi, L.: Hydrogen permeation behaviour in austenitic stainless steels. In: Materials Science and Engineering: A, 114, pp. 179-187 (1989)

[207] Wagner, S., Pundt, A.: Electrical resistivity and hydrogen solubility of PdHc thin films. In: Acta Materialia, 58:4, pp. 1387-1394 (2010)

[208] Goldstein, J. I., Newbury, D. E., Michael, J. R., Ritchie, N. W., Scott, J. H. J., Joy, D. C.: Scanning Electron Microscopy and X-Ray Microanalysis. Springer New York (2017)

[209] Williams, D. B., Carter, C. B.: Transmission Electron Microscopy. Springer (1996)

[210] Hamm, M., Bongers, M. D., Roddatis, V., Dietrich, S., Lang, K.-H., Pundt, A.: In situ hydrogen loading of magnesium thin-films observed with environmental TEM. In: submitted (2019)

[211] Schindelin, J., et al.: Fiji: an open-source platform for biological-image analysis. In: Nature Methods, 9:7, pp. 676-682 (2012)

[212] Paik, B., Jones, I., Walton, A., Mann, V., Book, D., Harris, I.: $M g H$ $2 \rightarrow M g$ phase transformation driven by a high-energy electron beam: An 
in situ transmission electron microscopy study. In: Philosophical Magazine Letters, 90:1, pp. 1-7 (2010)

[213] Kapur, J., Sahoo, P., Wong, A.: A new method for gray-level picture thresholding using the entropy of the histogram. In: Computer Vision, Graphics, and Image Processing, 29:3, pp. 273-285 (1985)

[214] Egerton, R.: Electron Energy-Loss Spectroscopy in the Electron Microscope. Springer Science \& Business Media (2011)

[215] Spiess, L., Teichert, G., Schwarzer, R., Behnken, H., Genzel, C.: Moderne Röntgenbeugung: Röntgendiffraktometrie für Materialwissenschaftler, Physiker und Chemiker (German Edition). Vieweg+Teubner Verlag (2009)

[216] Bragg, W. L.: The Structure of Some Crystals as Indicated by Their Diffraction of X-rays. In: Proc. Roy. Soc. A, volume 89, pp. 248-277. The Royal Society (1913)

[217] Scherrer, P.: Bestimmung der Größe und der inneren Struktur von Kolloidteilchen mittels Röntgenstrahlen. In: Nachrichten von der Gesellschaft der Wissenschaften zu Göttingen, Mathematisch-Physikalische Klasse, pp. 98-100 (1918)

[218] Langford, J. I., Wilson, A. J. C.: Scherrer after sixty years: A survey and some new results in the determination of crystallite size. In: Journal of Applied Crystallography, 11:2, pp. 102-113 (1978)

[219] C. Suryanarayana, M. G. N.: X-ray diffraction: a practical approach. Springer Us (2013)

[220] Deslattes, R. D., Kessler, E. G., Indelicato, P., de Billy, L., Lindroth, E., Anton, J.: X-ray transition energies: new approach to a comprehensive evaluation. In: Reviews of Modern Physics, 75:1, pp. 35-99 (2003)

[221] Hamm, M., Pundt, A.: FEM simulation supported evaluation of a hydrogen grain boundary diffusion coefficient in $\mathrm{MgH}$ 2. In: International Journal of Hydrogen Energy, 42:35, pp. 22530-22537 (2017)

[222] Jain, S. C., Harker, A. H., Cowley, R. A.: Misfit strain and misfit dislocations in lattice mismatched epitaxial layers and other systems. In: Philosophical Magazine A, 75:6, pp. 1461-1515 (1997)

[223] Yu, Q., Qi, L., Mishra, R. K., Zeng, X., Minor, A. M.: Size-dependent 
mechanical properties of $M g$ nanoparticles used for hydrogen storage. In: Applied Physics Letters, 106:26, pp. 261903 (2015)

[224] Teichmann, N., Hamm, M., Pundt, A.: Fast lateral hydrogen diffusion in magnesium-hydride films on sapphire substrates studied by electrochemical hydrogenography. In: International Journal of Hydrogen Energy, 43:3, pp. 1634-1642 (2018)

[225] Mooij, L., Dam, B.: Hysteresis and the role of nucleation and growth in the hydrogenation of $\mathrm{Mg}$ nanolayers. In: Physical Chemistry Chemical Physics, $15: 8$, pp. $2782(2013)$

[226] Hadjixenophontos, E., Roussel, M., Sato, T., Weigel, A., Stender, P., ichi Orimo, S., Schmitz, G.: Imaging the hydrogenation of $M g$ thin films. In: International Journal of Hydrogen Energy, 42:35, pp. 22411-22416 (2017)

[227] Ostrovsky, A.: Grain Boundary Diffusion in Thin Films under Stress Field in Kinetic Regime C. In: Defect and Diffusion Forum, 156, pp. 249-254 (1998)

[228] Nörthemann, K., Pundt, A.: Coherent-to-semi-coherent transition of precipitates in niobium-hydrogen thin films. In: Physical Review B, 78:1, pp. 014105 (2008)

[229] Mezbahul-Islam, M., Mostafa, A. O., Medraj, M.: Essential Magnesium Alloys Binary Phase Diagrams and Their Thermochemical Data. In: Journal of Materials, 2014, pp. 1-33 (2014)

[230] Boulet, J., Gerard, N.: The mechanism and kinetics of hydride formation in Mg-10wt.\% Ni and CeMg12. In: Journal of the Less Common Metals, 89:1, pp. 151-161 (1983)

[231] Mintz, M. H., Malkiely, S., Gavra, Z., Hadari, Z.: Effect of group IIIA metal additives on the kinetics of magnesium hydride formation. In: Journal of Inorganic and Nuclear Chemistry, 40:11, pp. 1949-1951 (1978)

[232] Völkl, J., Alefeld, G.: Hydrogen diffusion in metals. In: Nowick, A. (Hrsg.), Diffusion in solids: recent developments, chapter 5, p. 231ff. Academic Press New York (1975)

[233] Flanagan, T. B., Oates, W. A.: The Palladium-Hydrogen System. In: Annual Review of Materials Science, 21:1, pp. 269-304 (1991)

[234] Nowotny, M., Bak, T., Nowotny, J., Sorrell, C.: Titanium vacancies in 
C. Bibliography

nonstoichiometric TiO2 single crystal. In: physica status solidi (b), 242:11 (2005) 


\title{
D. Danksagung
}

\author{
Es ist immer klüger, seine Unkenntnis zuzugeben und Fragen zu stellen als aus \\ falschem Stolz blind vorwärts zu stolpern \\ (Jean-Luc Picard, Star Trek: The Next Generation)
}

An dieser Stelle möchte ich mich bei allen bedanken die mir erlaubt haben diese Arbeit durchzuführen und erfolgreich abzuschließen. Dies ist besonders Prof. Dr. Pundt die mich in den letzten sieben Jahren immer hervorragend (an-)geleitet hat. Unter dieser Leitung habe ich gelernt effektiv und trotzdem kreativ zu forschen. Ihre Bereitschaft zu diskutieren und dabei jeden sich einbringen zu lassen war enorm wertvoll für mich

Daneben möchte ich Prof. Dr. Reiner Kircheim dafür danken, dass er das Korreferats dieser Arbeit übernommen hat. Den Professoren Prof. Dr. Cynthia Volkert, Prof. Hans-Christian Hofsäss, Prof. Dr. Vasily Moshnyaga und Prof. Dr. Michael Seibt danke ich dafür, dass sie sich bereit erklärt haben Teil meiner Prüfungskommission zu sein.

Ich möchte mich bei den aktuellen und ehemaligen Mitgliedern der Arbeitsgruppe Pundt bedanken. Mein Dank geht an Dr. Stefan Wagner der mich schon als Bachelor betreute und auch danach immer wertvolles Feedback gab. Dr. Vladimir Burlaka betreute mich während meiner Masterarbeit und wir waren immer eine gutes Team, bei verschiedensten Experimenten und Messreisen. Dr. Helmut Uchida hat die Grundlagen zu dieser Arbeit gelegt. Sein umfangreichstes Wissen über Magnesium war mir eine große Hilfe einen Einstieg in das Thema zu finden. Dieses Projekt wäre nie möglich ohne seine Arbeiten, dafür bedanke ich mich. Ich danke Dr. Ryota Gemma und Dr. Chika Izawa für die freundliche Unterstützung zu Beginn meiner Zeit in der Arbeitsgruppe Pundt. Weiter danke ich Dr. Marian Bongers, meinem langjährigen Büropartner. Unser täglicher Austausch war äußerst wichtig für mich und ich hoffe auch für ihn. Ich danke Anshu Tyagi für gute Zusammenarbeit in den letzten Jahren. Des weiteren danke ich den aktuellen und ehemaligen Bachelor- und Masterstudenten welche ich betreuen durfte. Philipp Klose, Niklas Teichmann, Felix Jung und Felix Maischner haben wichtige Beiträge zu meinen Arbeiten geleistet (der vorliegenden sowie, im Falle von Philipp, zu den Arbeiten an Niob). Ich erwarte in 


\section{Danksagung}

Zukunft von Ihnen zu hören. Ich danke auch meinen anderen Bachelorstudenten Jantje Lina Schommartz, Svenja Willig und Melissa Schenker.

Weiter möchte ich mich noch bei unseren ,adoptierten“ Gruppenmitgliedern (aus der Arbeitsgruppe Kirchheim) Dr. Marie Tiegel und Dr. Martin Deutges, sowie Dr. May Ling Martin bedanken. Alle drei haben mir tagtäglich geholfen, sei es bei meinen Experimenten als auch als stetige Diskussionspartner.

Mein Dank gilt ebenfalls der ehemaligen Arbeitsgruppe Krebs. Dr. Felix Schlenkrich und Dr. Florian Döring danke ich für die Präparation der ersten dünnen PLD Magnesium Schichten. Besonders danke ich Prof. Dr. Hans-Ulrich Krebs für die Unterstützung der Arbeit und den anregenden Diskussionen. Dr. Sarah Hoffmann-Urlaub danke ich für die Fortsetzung der Arbeit der Gruppe Krebs und für die Präparation der endgültigen Schichten. Diese waren fundamental für meine Diffusionsmessungen und diese Arbeit.

Tobias Schulz war mir eine große Hilfe bei allen technischen Problemen, was ich auch täglich genutzt habe. Auch den anderen Techniker des Institutes, vor allem Karin Ahlborn, Kerstin Born, Frank Köhler und allen Mitgliedern der Elektronik Werkstatt danke ich. Sie waren immer freundlich und hilfsbereit egal wie oft ich sie in Beschlag nehmen musste. Volker Radisch war mir eine große Hilfe bei den REM und FIB Arbeiten. Vladimir Roddatis und Mathias Hahn waren unerlässliche Hilfen bei meinen TEM Experimenten, besonders dem in-situ Experiment. Dieter Plischke und Conny Mewes danke ich für die Hilfe und Betreuung bei den Röntgen Experimenten. Für die Unterstützung bei allen Sachen IT danke ich Michael Malchow. Diesen und allen weiteren Technikern und wissenschaftlichen Mitarbeitern des IMP danke ich, ohne sie wäre keine erfolgreiche Forschung möglich.

Ich danke den Betreuern der Beamlines am DESY, Hamburg und am ESRF, Grenoble Dr. Carsten Baetz und Dr. Olliver Seeck für die fundamentale Unterstützung der Synchrotron Experiments.

Frau Christina Kuba, Regina Wunderlich, Karin Haacke und Phuong Quan danke ich für die Unterstützung bei allen administrativen Dingen. Sie haben mich immer freundlich und kompetent durch alle Anträge geleitet.

Abschließend möchte ich meiner Mutter Sybille Werres-Hamm, meinem Bruder Laurits Hamm und meiner Freundin Marlen Berger danken. Ihr habt mich in all den Jahren unterstützt und mir mehr geholfen, als ihr vielleicht selber wisst.

Ich entschuldige mich bei all den anderen Menschen die mich sonst gefördert, gefordert und getrieben haben. Ich kann euch nicht alle namentlich aufführen, also: 
Machts gut und danke für den Fisch ${ }^{1}$

Diese Arbeit wurde von der DFG über die Projekte PU131/10-1 and PU131/10-2 finanziell großzügig gefördert.

${ }^{1}$ Douglas Adams, Per Anhalter durch die Galaxis 